\title{
WestVirginiaUniversity
}

THE RESEARCH REPOSITORY @ WVU

Graduate Theses, Dissertations, and Problem Reports

2001

\section{Damage mechanics of human cortical bone}

\author{
Gagik Parsam Parsamian \\ West Virginia University
}

Follow this and additional works at: https://researchrepository.wvu.edu/etd

\section{Recommended Citation}

Parsamian, Gagik Parsam, "Damage mechanics of human cortical bone" (2001). Graduate Theses, Dissertations, and Problem Reports. 2367.

https://researchrepository.wvu.edu/etd/2367

This Dissertation is protected by copyright and/or related rights. It has been brought to you by the The Research Repository @ WVU with permission from the rights-holder(s). You are free to use this Dissertation in any way that is permitted by the copyright and related rights legislation that applies to your use. For other uses you must obtain permission from the rights-holder(s) directly, unless additional rights are indicated by a Creative Commons license in the record and/ or on the work itself. This Dissertation has been accepted for inclusion in WVU Graduate Theses, Dissertations, and Problem Reports collection by an authorized administrator of The Research Repository @ WVU.

For more information, please contact researchrepository@mail.wvu.edu. 


\title{
Damage Mechanics of Human Cortical Bone
}

\author{
Gagik P. Parsamian
}

\author{
Dissertation submitted to the \\ College of Engineering and Mineral Resources \\ at West Virginia University \\ in partial fulfillment of the requirements \\ for the degree of
}

Doctor of Philosophy

in

Mechanical Engineering

\author{
Timothy L. Norman, Ph.D., Chair \\ Ever J. Barbero, Ph.D. \\ Julio F. Davalos, Ph.D. \\ Thomas A. Gruen, M.S. \\ Nilay Mukherjee, Ph.D.
}

Department of Mechanical and Aerospace Engineering

Morgantown, West Virginia

2001

Keywords: Bone, Microdamage, Model, Viscoelasticity, Damage Mechanics 


\section{ABSTRACT \\ Damage Mechanics of Human Cortical Bone}

\section{Gagik P. Parsamian}

Skeletal fragility is an important orthopedic concern including the prevention of osteoporosis, long-term stability of prosthetic implants and stress fractures. Damage in human cortical bone has been implicated as a cause of increased fragility and is thought to initiate bone remodeling. Therefore, characterization of the mechanisms of damage initiation and accumulation in bone is challenging not only from the engineering prospective but also has a potential of revealing new insights in its physiology. The main objectives of this dissertation work were to study the early stages of damage development in human cortical bone and develop a constitutive formulation describing damage behavior.

Laser Scanning Confocal Microscopy was utilized to study damage genesis. It was found that confocal microscopy allowed detection of the early stages of damage development within the lacunae-canalicular network of cortical bone. Based on those observations and a common knowledge about the operation of mechanosensing cells housed within the tissue it was proposed that damage could initiate bone remodeling much earlier than it is currently believed.

In the second portion of this work the evolutionary properties of chosen damage parameters were investigated under 3-point bending loading. The parameters of interest were the stiffness drop and the permanent strain. It was found that shear stresses play profound role in the failure behavior. It was also shown that damage parameter defined as the stiffness drop after successive cycles is a quadratic function of nonlinear strain. In addition a linear relationship was obtained between the permanent and total strain.

The third portion of this work was concerned with the development and validation of a constitutive model for cortical bone based on the continuum damage mechanics. It was demonstrated that bone is a linear viscoelastic material for stress levels below a threshold value. Beyond the threshold it behaves as a viscoelastic damaging material. Subsequently a coupled viscoelasticity-damage formulation was adopted and a model was derived based on thermodynamics of irreversible processes. The model was simplified for one-dimensional uniaxial case and experiments were performed for the model verification. 


\section{ACKNOWLEDGEMENTS}

I would like to acknowledge a number of individuals who supported my efforts in completing this dissertation. First, I would like to express my sincere gratitude to my research advisor, Dr. Tim Norman, first for giving me this opportunity to be involved in such a fascinating field of research as bone mechanics and for his consistent encouragement, motivation and guidance throughout the years of this research work. I would like to thank the entire team of committee members of this project: Dr. Ever Barbero, for teaching me the fundamentals of rather complex theories, including damage mechanics, which served the core of this research; Mr. Tom Gruen, for countless discussions, critical remarks and his great friendship; Dr. Nilay Mukherjee, for his boundless interest in science and Dr. Julio Davalos, for his crucial remarks and guidance.

I would like to express my special gratitude to my friend and colleague, Vince Kish for his patience and support and for making me appreciate the experimental work. None of the experimental studies would have been possible without his help. I would also like to thank everyone at Musculoskeletal Research Center of the Department of Orthopedics (Nina Clovis, Suzanne Smith, Chris Brown and Keith Hustosky) for their friendship, helpfulness and their unique ability to generate a working atmosphere filled with joy.

And most importantly, I would like to thank my wife Zara for her patience, love and understanding and for giving me boundless happiness by blessing me with our newborn son, Mark. 


\section{TABLE OF CONTENTS}

CHAPTER 1.INTRODUCTION

1.1. Introduction

1.2. Function of Bone ..................................................................................... 1

1.3. Structure of Bone ............................................................................................. 3

1.4. Bone Adaptation to Mechanical Stimuli................................................................ 4

1.5. Mechanotransduction in Bone ................................................................. 5

1.6. Material Models for Bone .......................................................................... 7

1.6.1.Particulate Composite Models ...................................................................... 7

1.6.2. Fiber Composite Theories...................................................................... 8

1.6.3.Osteonal Modeling .............................................................................. 9

1.7. Elastic Properties of Bone .............................................................................. 10

1.8. Viscoelasticity of Bone .............................................................................. 12

1.9. Bone Poroelasticity .................................................................................... 13

1.9.1. Structural interfaces of bone ................................................................. 14

1.9.2. Porosities of Bone .......................................................................... 15

1.10. Fracture Mechanics of Bone ....................................................................... 16

1.11. Summary ..................................................................................... 18

1.12. The Overall Objectives of This Dissertation Work ........................................... 19

CHAPTER 2. INITIAL STAGES OF DAMAGE DEVELOPMENT IN HUMAN

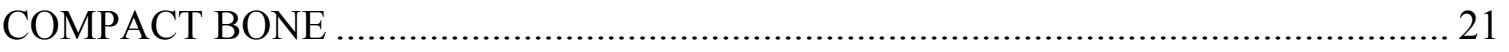

2.1. Introduction..................................................................................... 21

2.1.1. Fatigue of Bone ................................................................................ 24

2.1.2. Bone Microdamage ............................................................................ 26

2.1.3. Confocal Microscopy ......................................................................... 28

2.1.4. Objectives .................................................................................... 29

2.2. Materials and Methods............................................................................. 31

2.2.1. Specimen Preparation ......................................................................... 31

2.2.2. Mechanical Testing .............................................................................. 35

2.3. Results..................................................................................... 37

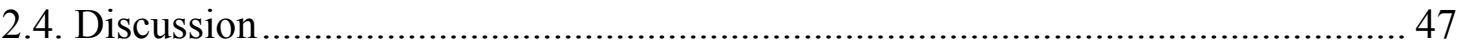


CHAPTER 3. DAMAGE DEVELOPMENT IN 3-POINT BENDING OF HUMAN

CORTICAL BONE.................................................................................... 51

3.1. Introduction ............................................................................................. 51

3.2. Objectives ..................................................................................... 55

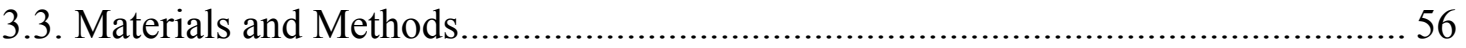

3.3.1. Specimen Preparation ..................................................................... 56

3.3.2. Mechanical Testing .......................................................................... 56

3.4. Results........................................................................................ 60

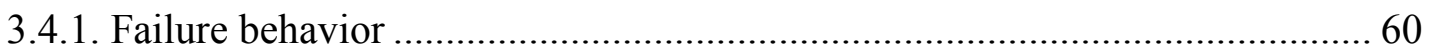

3.4.2 Damage Evolution ................................................................................ 64

CHAPTER 4. CONTINUUM DAMAGE MODEL FOR HUMAN CORTICAL BONE 71

4.1. Introduction..................................................................................... 71

4.2. Fundamentals of Classical Thermodynamics ...................................................... 72

4.2.1 First Law of Thermodynamics (Conservation of Energy) ............................. 72

4.2.2. The Second Law of Thermodynamics ...................................................... 73

4.2.3. Thermodynamics of irreversible processes................................................ 74

4.3. Review of pertinent literature on CDM theory .............................................. 76

4.4. Continuum Damage Model for human cortical bone ......................................... 78

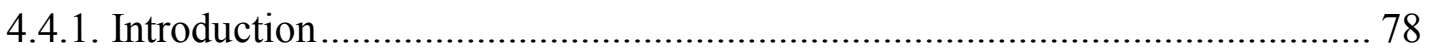

4.4.2. General Thermodynamic Considerations...................................................... 79

4.4.3. Viscoelasticity Coupled with Damage .......................................................... 82

4.4.4. Damage Evolution Law ....................................................................... 88

4.4.5. Viscoelastic Compliance Tensor............................................................... 91

4.5. Objectives .................................................................................................. 93

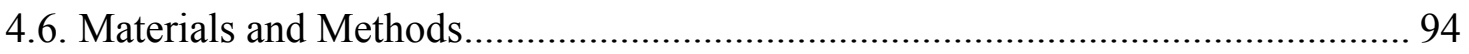

4.6.1. Specimen Preparation ........................................................................... 94

4.7. Experimental Methods for Determination of Threshold Stress .......................... 97

4.7.1. Results and Discussion ............................................................................ 100

4.7.2. Summary …….......................................................................... 114

4.8. Experimental Methods for Determination of the Viscoelastic Compliance of the

Virgin Material. .......................................................................................................... 115 


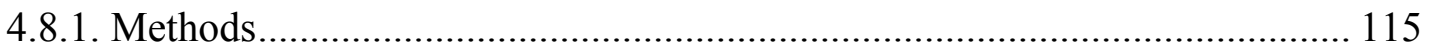

4.8.2. Results ........................................................................................ 115

4.8.2. Summary ......................................................................................... 119

4.9. Experimental Methods for Studying Creep Rupture and Determination of Critical

Damage Parameters. .................................................................................... 119

4.9.1. Introduction ..................................................................................... 119

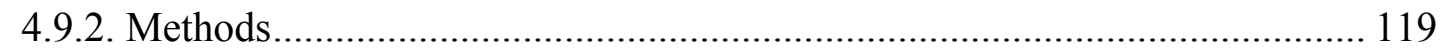

4.9.3. Results and Discussion ..................................................................... 120

4.10. Theoretical Predictions ...................................................................... 125

4.11. Validation of the Model .............................................................................. 132

4.11.1.Experimental Methods .......................................................................... 132

4.11.2. Results and Discussion .................................................................... 132

4.12. Limitations of the Model .............................................................................. 141

4.13. Conclusions............................................................................................. 142

CHAPTER 5. CONCLUSIONS AND FUTURE DIRECTIONS .............................. 143

CHAPTER 6. REFERENCES ....................................................................... 146 


\section{LIST OF FIGURES}

Figure 1.1. Schematic diagram of cortical bone structure (from Kessel and Kardon,

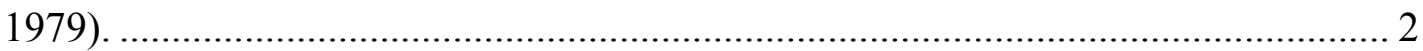

Figure 1.2. The hierarchy of structure of cortical bone (from Lakes, 1993). .................... 4 Figure 1.3. Enlargement of lacunar-canalicular system. Arrows indicate the gap junctions between osteocytic processes (from Weinbaum et al., 1998)................................. 5 Figure 1.4: Bone lining cells and osteocytes within fluid saturated bone matrix ............. 14 Figure 2.1. Schematic representation of the bone remodeling sequence. The resorption (R) sequence (1 - 3): 1) osteoclastic phase; 2) mononuclear phase; 3) preosteoblastic phase. The Formation (F) sequence (4 - 6): 4) unmineralized matrix (osteoid); 5) mineralization; 6) formation of bone lining cells (final thickness) (Frost, 1963a)...22

Figure 2.2. Schematic diagram showing how osteonal debonding may initiate BMU activation. Crack around the cement line creates a disuse state, which leads to bone absorption, followed by bone deposition. As a result crack could be repaired by the

newly formed osteon........................................................................................ 24

Figure 2.3. An image of transverse section of human cortical bone stained with basic fucsin, demonstrating a typical microcrack in bone matrix..................................2 27

Figure 2.4. Schematic diagram of a typical LSCM system. ........................................ 29

Figure 2.5a. Compact Tension specimens were machined in pairs from anteromedial, anterolateral and posterior cortices of human tibial sections, obtained from distal, medial and proximal locations......................................................................... 32

Figure 2.5b. Final geometry of specimens with $B=2 \mathrm{~mm}$ and $a / W=0.5$. An area of

$921.3 \times 921.3 \mu \mathrm{m}^{2}$ was investigated for damage development. Objectives with higher magnification allowed further subdivision of this area into 4 and 16 fields for more detailed observations. A $250 \mu \mathrm{m}$ slice, $1 \mathrm{~mm}$ away from the chosen area of damage observations, was cut for morphologic analysis of bone microstructure.................. 33 Figure 2.6. An image of a LSM 510 software window with an open image of bone section with a circumscribed diffuse damage area............................................................ 35

Figure 2.7. Typical micrograph demonstrating the longitudinal (A) and transverse (B) appearance of bone under LSCM. .................................................................... 38 
Figure 2.8. LSCM micrograph of the specimen surface oriented longitudinally to the direction of osteons with a tensile loading axis perpendicular to the horizontal direction. A typical appearance of diffusely damage areas demonstrating damaged regions in the canalicular network of lacunae (the scale bar is $30 \mu \mathrm{m}$ long)............ 39

Figure 2.9. A confocal micrograph demonstrating pooled regions of stain in the intercanalicular spaces in-between neighboring lacunae. ............................... 40 Figure 2.10. Confocal micrograph demonstrating the path of a crack with multiple deflections passing through a lacuna and passing around another one. This behavior is an indicator of the stress concentrating effects of lacunae (the scale bar is $20 \mu \mathrm{m}$

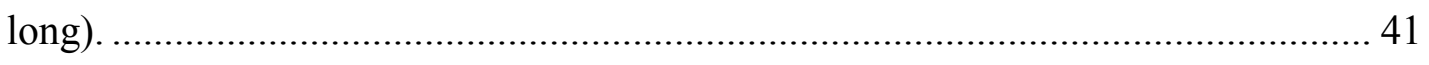

Figure 2.11. A confocal micrograph demonstrating the overlapping nature of

microcracking, similar to other engineering materials that experience toughening

behavior as a result of long discretized microcracks (the scale bar is $40 \mu \mathrm{m}$ long). 42

Figure 2.12. A graph representing a typical load vs. displacement relationship during monotonic loading of CT specimens. $\mathrm{P}_{\mathrm{C}}$ was found by superimposing a line parallel to the initial linear portion of the curve and having a $0.02 \%$ offset and determining the point of intersection with the load-displacement curve. .................................4 43 Figure 2.13. Graph representing the relationship between the fracture toughness $\left(\mathrm{K}_{\mathrm{IC}}\right)$ and diffuse damage (Df.Dm.D). The relationship is nonlinear and is given by:............44

Figure 2.14a. Analysis of variance of Os.D. as a function of bone location and bone

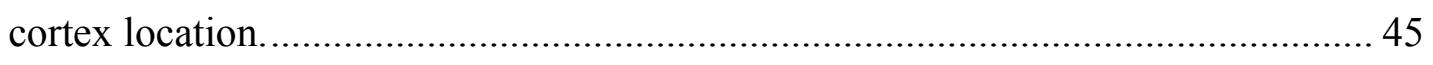

Figure 2.14b. Analysis of variance of $\mathbf{P}$ as a function of bone location and bone cortex

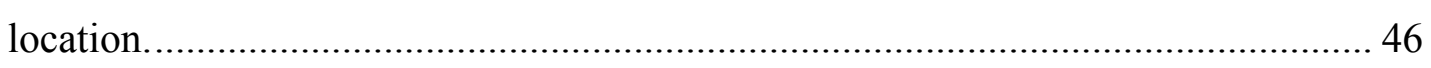

Figure 3.1. Typical stress-strain behavior of elastic, plastic, visoelastic and viscoplastic materials. In elastic materials unloading follows the same loading path. In plastic materials material unloads with the same modulus leading to unrecoverable plastic residual strain. In viscoelastic materials residual strains completely recover upon removal of load. In viscoplastic materials residual strains do not remain recover completely upon removal of load ................................................................ 52 
Figure 3.2. Stress strain relationships for plastic and pseudoplastic materials. Plastic

behavior demonstrates characteristic residual strains after unloading, whereas

pseudoplastic material unloads to its original configuration. ............................ 53

Figure 3.3. Schematic diagram of 3-point bending fixture indicating the locations of

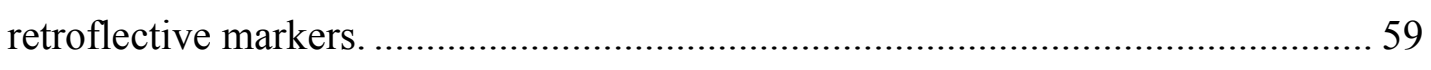

Figure 3.4. Confocal micrographs demonstrating nondamaged lacuna-canalicular

network(left) and damaged canalicular network............................................. 60

Figure 3.5 Typical appearance of compressive (left) damage and tensile (right) damage.

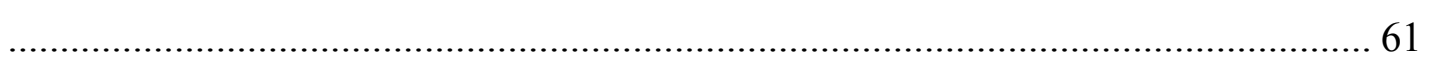

Figure 3.6. Areas of diffuse damage in the matrix region. ................................... 61

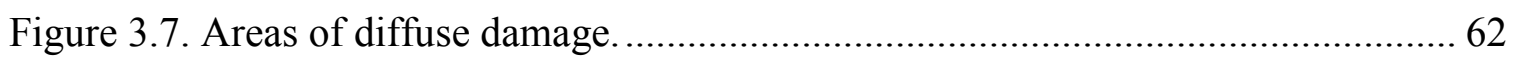

Figure 3.8 A superimposed image obtained by LSCM and light microscope. ................ 62

Figure 3.9 Confocal micrograph of sections from an entire cross-section of specimen

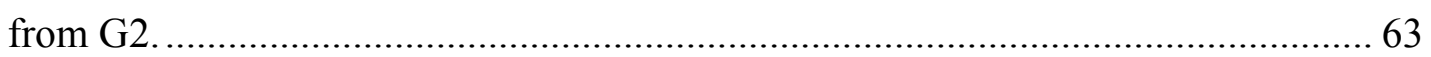

Figure 3.10 Schematic diagram of three major modes of failure under 3-point bending

conditions................................................................................................. 64

Figure 3.11. Stress strain history of specimen RDL02 from G1............................... 65

Figure 3.12 Stress-strain history of specimen RDP04 from G2............................ 65

Figure 3.13. Damage Evolution of specimens from G1. ...................................... 66

Figure 3.14 Damage Evolution of specimens from G2 ............................................. 66

Figure 3.15. The observed relationship between the ratio of residual and total strains as a

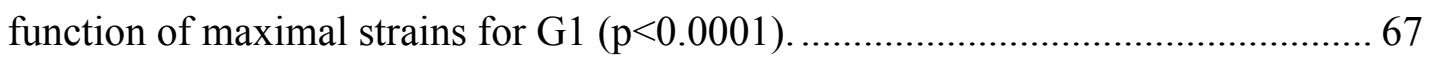

Figure 3.16. The observed relationship between the ratio of residual and total strains as a

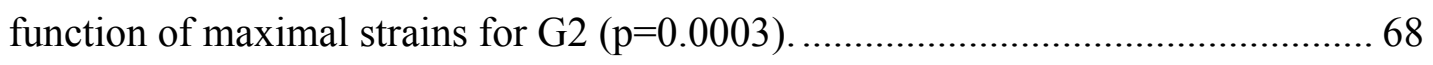

Figure 3.17. Analysis of variance (ANOVA) have demonstrated that the linear fit is

statistically significant fit of data given in Figure 3.15 for the G1......................6. 69

Figure 3.18. Analysis of variance (ANOVA) have demonstrated that the linear fit is

statistically significant fit of data given in Figure 3.16 for the G2 …................... 70

Figure 4.1. Effective stress and the equivalence of strain.................................... 77

Figure 4.2. Schematic diagram of expected behavior demonstrating typical creep and

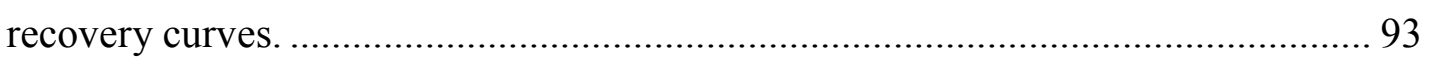


Figure 4.3. Specimen geometry......................................................................... 94

Figure 4.4. A photograph of a bone specimen. …………........................................ 95

Figure 4.5 Schematic diagram of the experimental setup............................................ 97

Figure 4.6. A photograph of a specimen in fixture with an attached extensometer. ........ 98

Figure 4.7. Typical loading history for the specimens from the group T. Figure on the

fight is an expanded view of left graph............................................................ 99

Figure 4.8. This graph represents the relationship between the extensometer reading in

volts and the corresponding deflections in inches. ............................................... 99

Figure 4.9. The stress-strain curves corresponding to the 17 loading ramps (specimen

\#T5). The plots B and C are the reproduction of the first plot (A) with shifted strains

(1000 and $10000 \mu$ srtains respectively) for the visual clarity purposes.................. 101

Figure 4.10 Stress-strain curves corresponding to 8 loading cycles (specimen\#T1). The

$2^{\text {nd }}$ plot is the reproduction of the first one with the initial strains shifted by

500ustrains................................................................................................ 102

Figure 4.11. Stress-Strain curves of first 5 cycles of the specimen\#T2 demonstrating

closed hysteretic loops. ............................................................................... 105

Figure 4.12. Strain vs. time curves produced during the successive loading cycles

superimposed on the first loading cycle duration. The 2nd plot is the reproduction of

the $1^{\text {st }}$ one with shifted initial strains for visual clarity......................................... 106

Figure 4.13. (a) Linear portion of the loading part of ramp\#10 of specimen\#T5 is fit

inearly to identify Young's modulus (in this case it is $\mathrm{E}=14.37 \mathrm{GPa}$ ); (b) The last 40

seconds of the hold part of the same ramp fit linearly to identify strain rate (in this

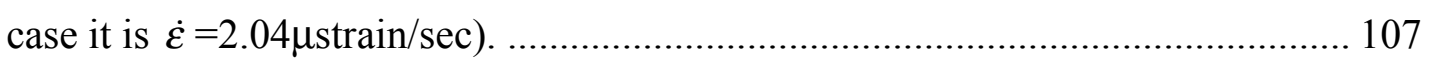

Figure 4.14. Typical relationships between the stiffness drop (plot A) and strain rate (plot

B) as a function of hold stress........................................................................ 109

Figure 4.15. The relationship between the stiffness drop and the hold stress for all the

specimens from tested at 5MPa/sec rate ........................................................... 110

Figure 4.16. The relationship between the strain rate and the hold stress of all the

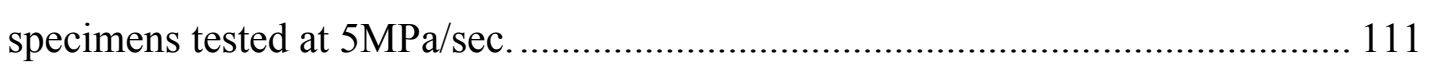

Figure 4.17. The final relationship between the strain rate and the hold stress of all the

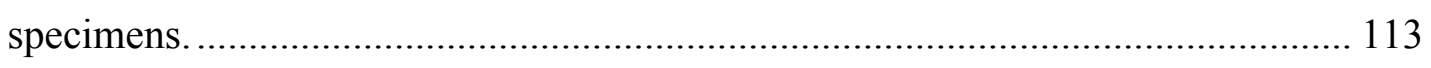


Figure 4.18. The result of the mean fit analysis performed on the initially linear portion of the data from the Figure 4.17....................................................................... 114 Figure 4.19 Nonlinear fit of the creep strain of the specimen T7 at a hold stress of

63.43MPa and duration of 60 seconds......................................................... 116 Figure 4.20 Nonlinear fit of the creep strain of the specimen T9 at a hold stress of $74.73 \mathrm{MPa}$ and duration of 60 seconds........................................................... 117

Figure 4.21. Nonlinear fit of the creep strain of the specimen T10 at a hold stress of 66.97MPa and duration of 60 seconds............................................................. 117 Figure 4.22. Nonlinear fit of the creep strain of the specimen T11 at a hold stress of 61.55MPa and duration of 60 seconds ........................................................ 118 Figure 4.23. Nonlinear fit of the creep strain of the specimen T12 at a hold stress of 59.66.MPa and duration of 60 seconds............................................................... 118 Figure 4.24 Creep curve of specimen R3 held at 82.4MPa........................................ 120 Figure 4.25 Creep curves of specimens R7 and R8, held at 87.9MPa and 98.5MPa,

respectively. ........................................................................................... 121

Figure 4.26. Creep curve of specimen R7 fit with Norton-Enquist equation with the

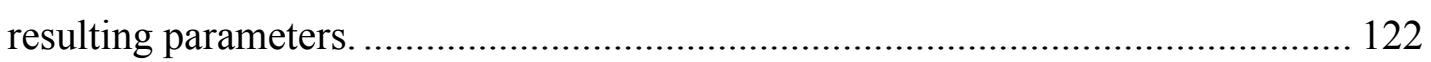

Figure 4.27. A graph representing the relationship between $\mathrm{t}_{\mathrm{F}}$ and $\left(\sigma-\sigma_{\mathrm{TH}}\right) \ldots \ldots \ldots \ldots \ldots \ldots . . . .123$

Figure 4.28. A graph representing the fit of time-to-failure vs. hold stress using Equation

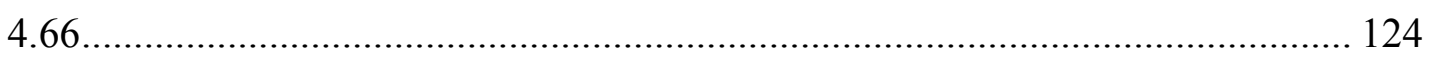

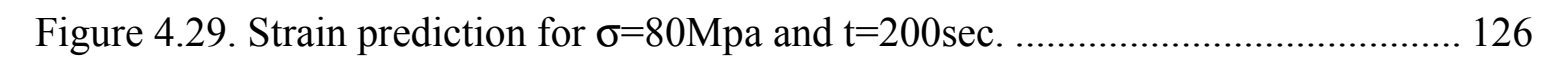
Figure 4.30. Strain prediction at the stress value approximately equal to the threshold value demonstrating the tertiary creep stage and hence the convergence at the infinity..................................................................................................... 127

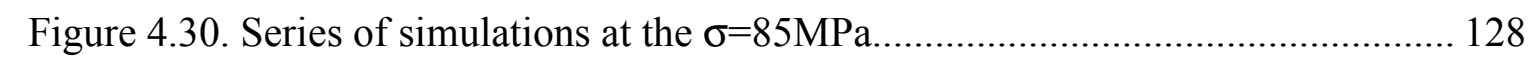

Figure 4.31. Predicted strain at $\sigma=85 \mathrm{MPa}$ with $\kappa=0.055$ and $\kappa=0.0929 \ldots \ldots \ldots \ldots \ldots \ldots . . .129$

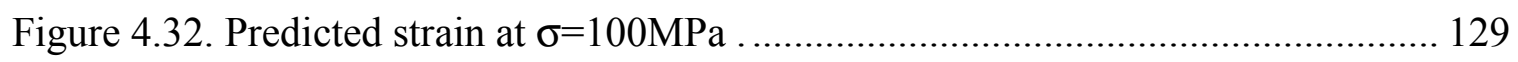

Figure 4.33. Series of simulations using Equation 4.74 at 50,60 and 70MPa and at $\sigma=100 \mathrm{MPa}$ for a long duration of time demonstrating inability to predict tertiary creep and failure........................................................................................................... 130 
Figure 4.34 simulation at identical loading conditions demonstrating the influence of $\mathrm{k}$

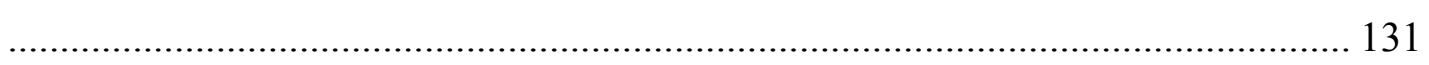

Figure 4.35 Strain recovery prediction using Equation 4.75 after hold stress of 70MPa for 60 seconds (plots 1 and 2 ) and hold stress of 60MPa for 60 seconds (plots 3 and 4 ).

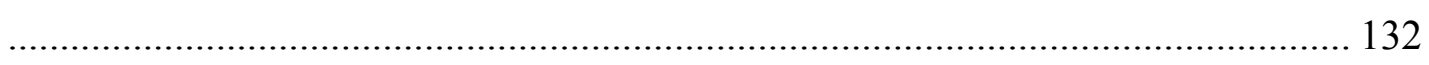

Figure 4.36 A graph representing the observed and predicted strains for specimen V1

held at 54MPa. ................................................................................................... 133

Figure 4.37 Experimental strain versus time for specimen V1 held at 82.4MPa for 60

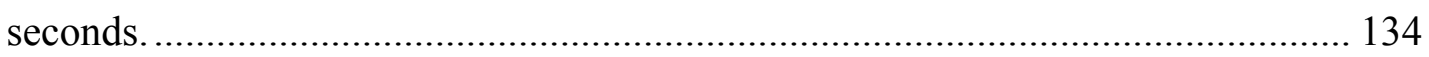

Figure 4.38 Strains predicted by the model for a specimen held at 82.4MPa for 60

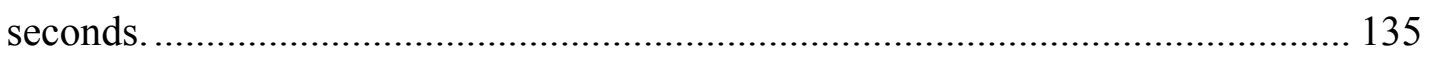

Figure 4.39. Strain versus time history observed and predicted for the specimen V2 at the

hold stress level of 67.4MPa and duration of 60seconds.................................... 136

Figure 4.40. Strain history until failure observed for specimen V2 held at 91.7MPa... 137

Figure 4.41. A series of simulations for the prediction of failure for a stress level of

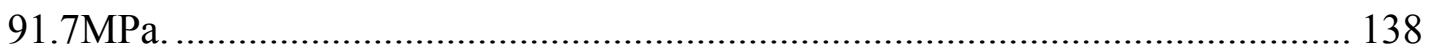

Figure 4.43 Strain versus time history observed and predicted for the specimen V3 at the

hold stress level of 58.5MPa and duration of 60seconds .................................... 139

Figure 4.44. Strain history until failure observed for specimen V3 held at 86.5MPa .... 139

Figure 4.45 Series of simulations for the prediction of failure for a stress level of

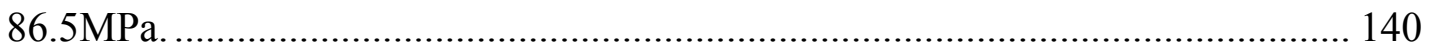

Figure 4.46. A graph representing the strain history observed for the specimen V4 tested

at 88.4MPa until failure together with predicted strains using the upper and lower

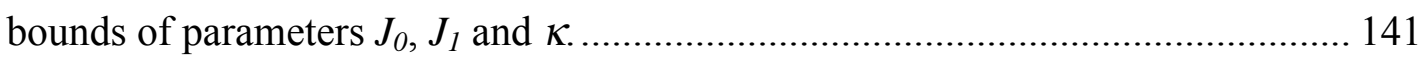




\section{LIST OF TABLES}

Table 1.1. Approximate sizes of fluid-filled regions of bone........................................ 6

Table 1.2. Material properties of the major components of bone ................................... 8

Table 1.3. Tensorial and technical elastic moduli of human cortical bone..................... 11

Table 1.4. Summary of fracture toughness values for human cortical bone (Norman et al.,

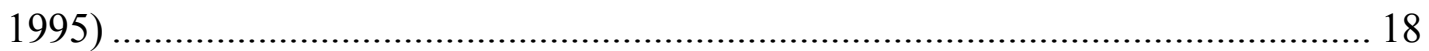

Table 2.1. Histomorphometric data for osteonal BMUs in human rib (Frost, 1969). ...... 23

Table 2.2. Microdamage data from various studies................................................. 28

Table 2.3. A list of specimens and their locations used for this study........................... 34

Table 3.1. Summary of specimens to be used in this portion of the study.................... 57

Table 4.1. Summary of specimens that were tested. $\mathrm{R}$ indicates creep rupture test

specimens and T-threshold tests specimens....................................................... 96

Table 4.2. Summary of the initial moduli of the specimens of the group T. .................. 107

Table 4.3 Summary of viscoelstic parameters obtained from short-term creep tests. .... 116

Table 4.4. A summary of specimens tested and the corresponding material parameters.

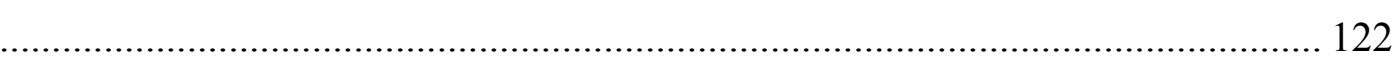




\section{CHAPTER 1 \\ INTRODUCTION}

\subsection{Introduction}

Understanding bone as a material is part of current goals of bone research with a main objective of obtaining increased knowledge of its physiology, which would ultimately lead to better description of its behavior under normal and pathological conditions. Assessing the mechanical consequences of bone diseases, which are accompanied with histological (microstructural) alterations, would be extremely beneficial in the overall understanding of bone physiology. This is different from the main goal of materials science and materials engineering, which is to try to enhance material performance and application for the design of structures by better understanding the material's behavior.

Damage description of a material requires: 1) understanding the physical mechanisms of microcracking, 2) defining the damage variable and formulating its evolution laws and 3) investigating the influence of damage on overall mechanical properties.

The goal of this study is to reveal the damage mechanisms of human cortical bone (Chapter 2), determine the damage evolution laws (Chapter 3) and propose a Continuum Damage Model (CDM) (Chapter 4) for human cortical bone.

\subsection{Function of Bone}

Bone is a living, metabolically active and highly organized tissue consisting of a mineral phase of hydroxyapatite (HA) and amorphous calcium phosphate crystals deposited in an organic matrix. It has two main functions: forms a rigid skeleton for load bearing and muscle attachments and has a central role in calcium and phosphate homeostasis. Throughout life it undergoes processes associated with growth and reshaping in childhood and adolescence, called bone modeling, and lifelong process whereby skeletal tissue is continuously being resorbed and replaced in order to maintain skeletal integrity, called remodeling. Bone remodeling is controlled by systematic hormones and cytokines and is an integral part of the calcium homeostatic system. 


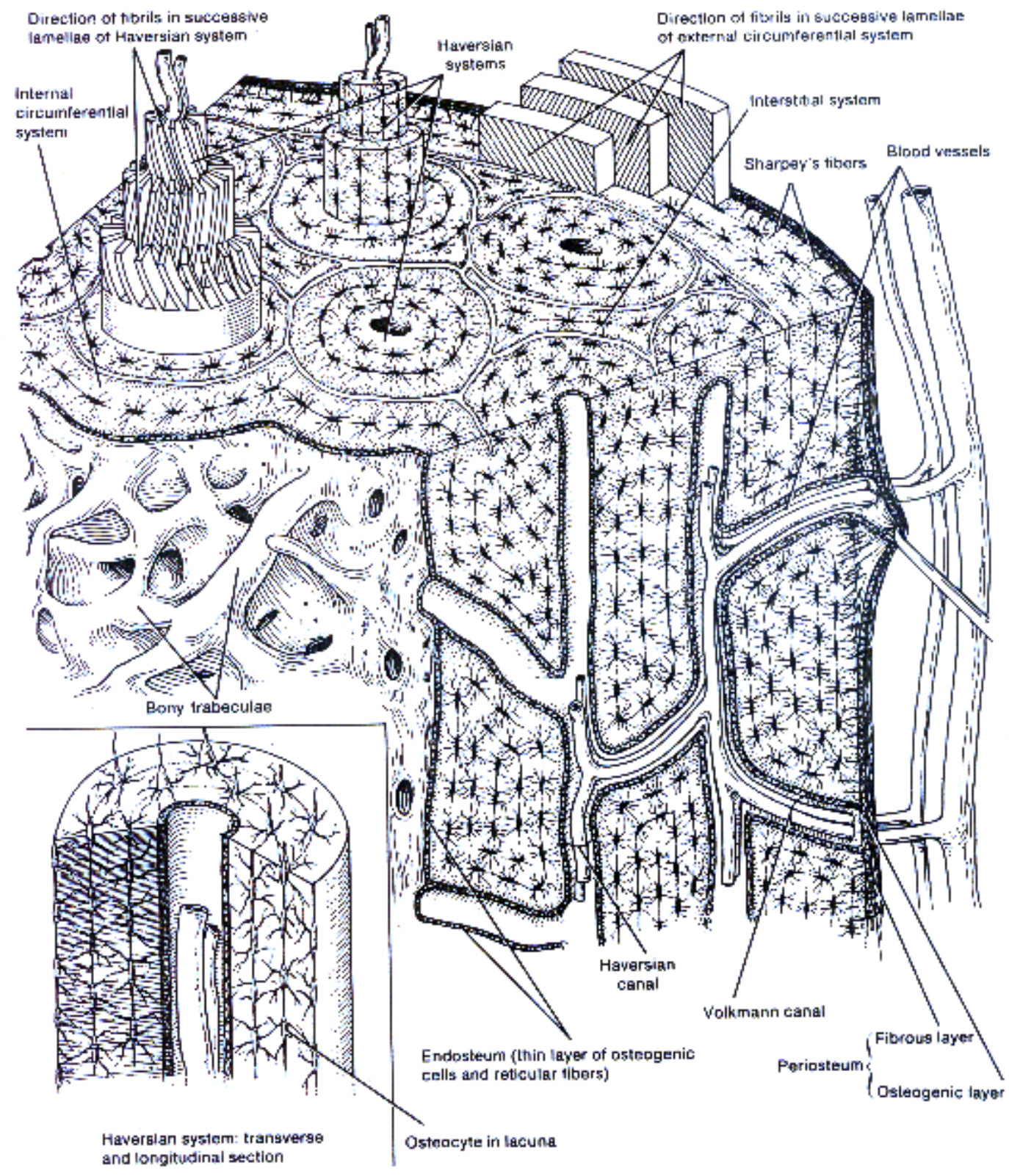

Figure 1.1. Schematic diagram of cortical bone structure (from Kessel and Kardon, 1979). 
The maintenance of a normal, healthy skeletal mass depends on interactions between osteoblasts (bone forming cells), osteoclasts (bone eating cells) and constituents of the bone matrix to keep the processes of bone resorption and formation in balance.

\subsection{Structure of Bone}

Bone has a varied arrangement of material structures at many length scales, which work in concert to perform diverse mechanical, biological and chemical functions (Figure 1.1). On the microstructural level are the osteons, which are relatively large hollow fibers composed of concentric lamellae and pores. The lamellae are built of fibers and the fibers contain fibrils. At the ultrastructural level (nanoscale) the fibers are composite of the mineral HA and the protein collagen (Figure 1.2). As the hierarchical order (Lakes, 1993; Rho et al., 1998) increases from the nanoscale, bone forms fairly distinct types: i) woven, lamellar and parallel-fibered bone; ii) fibrolamellar and Haversian bone (with primary and secondary osteons) and iii) compact (cortical) and cancellous bone (Currey, 1984).

Woven bone is characteristically produced during fetus and callus with randomly oriented, fine fibered collagen. Lamellar bone is more organized than woven bone consisting of $5 \mu \mathrm{m}$ thick sheets of lamellae with collagen having an almost perpendicular orientation with respect to the short axis of each lamellae (Boyde, 1980; Ascenzi et al., 1978) and is generally less mineralized than woven bone. Parallel-fibered bone is the intermediate configuration between woven and lamellar bone (Enlow, 1969; Ascenzi et al., 1967).

Primary osteons of Haversian bone are formed around Haversian canals by the deposition of concentric lamellae. This geometrical arrangement was speculated to facilitate the exchange of calcium ions (Ortner and Von Endt, 1971) and since the primary lamellar bone is some of the strongest bone in nature it was suggested that the formation of primary osteons might be functional (Carter and Spenger, 1978). Secondary osteons are formed as a result of the removal of primary bone by osteoclasts and redeposition by osteoblasts. As a result of this remodeling process secondary osteons form a cement line. It is believed that secondary osteons are formed to repair damaged (microcracked) bone (Burr et al., 1985). 


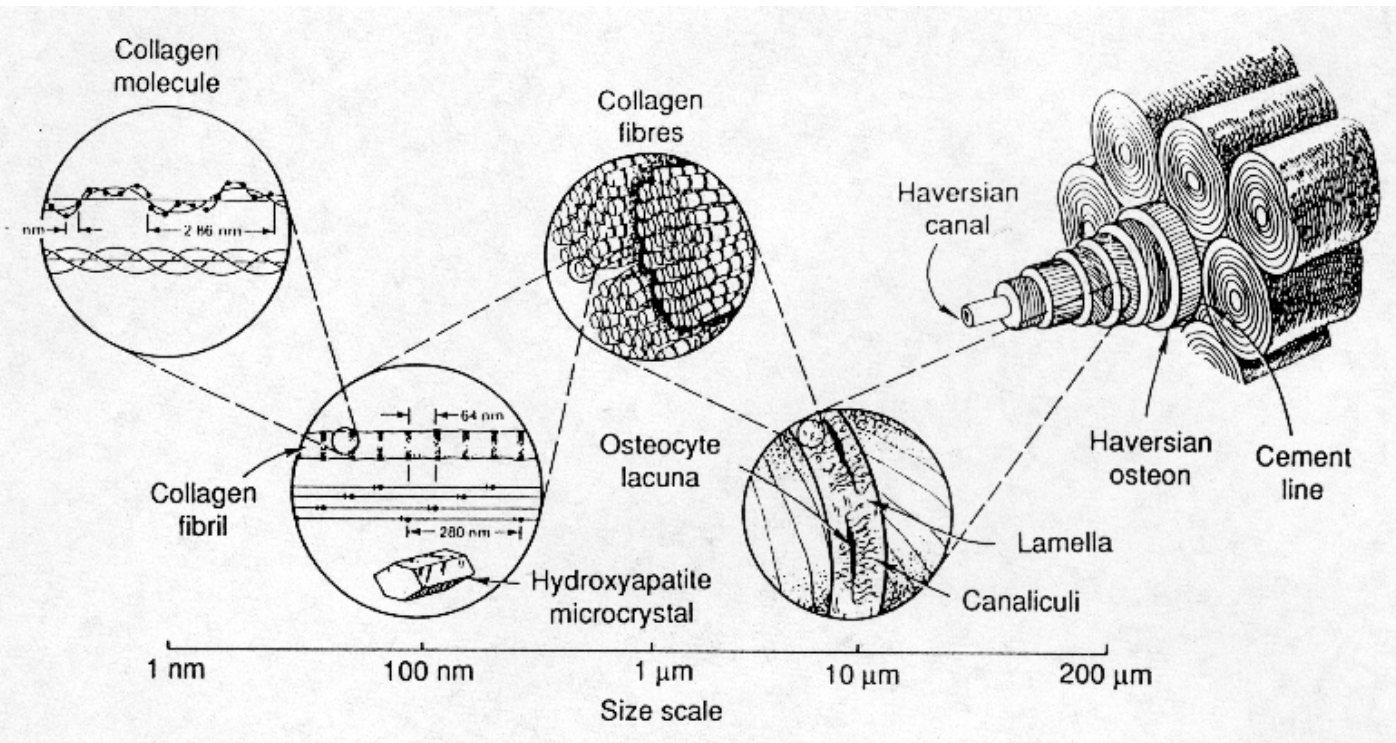

Figure 1.2. The hierarchy of structure of cortical bone (from Lakes, 1993).

Cortical bone is composed of approximately $30 \%$ water, $45 \%$ salts and $25 \%$ organic substance (Type I collagen (90\%) and amorphous ground substance (glycoproteins and glycosaminoglycans) (10\%)). To ensure tissue viability bone fluid, carrying requisite nutrition of the cells and essential disposal of their waste products must be able to flow through various microstructural spaces. Haversian and Volkman canals together with the lacunae-canalicular network serve as a network of channels for fluid transport.

\subsection{Bone Adaptation to Mechanical Stimuli}

Bone has an amazing ability to change its structure and morphology in response to alterations in the mechanical loading environment (Wolff 1892, 1986). These physiological processes of bone remodeling begin with the removal of bone by osteoclasts and are closely followed by the deposition of new bone by osteoblasts. Bone adaptation results in a change of the internal structure or the cortex and the alterations of its shape on the inner (endosteal) and outer (periosteal) surfaces. On the surface these processes are called "surface remodeling", defining the change in the bone crosssectional area. "Internal remodeling" on the other hand reflects the net change of material 
properties such as density, stiffness, etc. (Frost, 1964; Cowin and Hegedus, 1976; Hart and Davy, 1981).

A variety of stimuli have been considered for bone remodeling. The majority of researchers have followed Wolff (1892) and suggested that some aspect of the mechanical loading of bone is the stimulus. The mechanical stimuli suggested include strain (Cowin and Hegedus, 1976), stress (Wolff, 1892), strain energy (Fyhrie and Carter, 1986), strain rate (Lanyon, 1984) and fatigue microdamage (Frost, 1964; Carter and Hayes, 1976; Martin and Burr, 1982).

\subsection{Mechanotransduction in Bone}

The cellular level mechanisms, which enable cells enclosed in bone tissue osteocytes (Figure 1.3) to first sense mechanical loading of different frequency and magnitude, and secondly transmit this information to the bone lining osteoblasts producing new bone, is a question of debate.

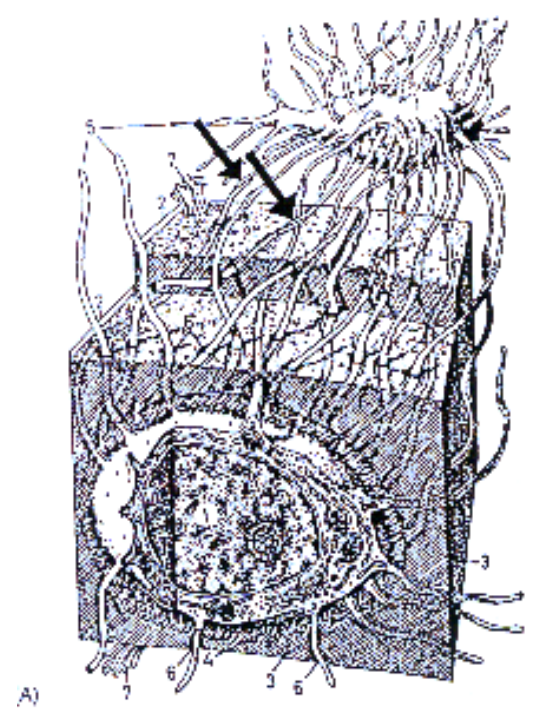

Figure 1.3. Enlargement of lacunar-canalicular system. Arrows indicate the gap junctions between osteocytic processes (from Weinbaum et al., 1998).

Four hypotheses have been proposed to explain the mechanisms by which cells sense mechanical loading: a piezoelectric effect; (Scott and Korostoff, 1990), stress generated potentials (SGP) (Salzstein et al., 1987; Salzstein and Pollak, 1987; Starkebaum et al., 
1979), a fluid shear stress on the bone lining cells in the vascular porosity (Hillsley and Frangos, 1994) and fluid shear stress on the membranes of the osteocytic processes in their canaliculi (Weinbaum et al., 1994).

Piezoelectric and electrokinetic mechanisms are two proposed mechanisms (Anderson and Eriksson, 1968) to explain electromechanical effects (potentials) first observed by Yasuda (1953). Electromechanical potentials often called stress-generated potentials are the voltages induced by mechanical deformation of fluid saturated bone.

Table 1.1. Approximate sizes of fluid-filled regions of bone.

\begin{tabular}{lll}
\hline Region & Shape & Size \\
\hline Lacunae & ellipsoidal & $5-10 \mu \mathrm{m}$ (major axis) \\
Canaliculus & cylindrical & $\approx 200 \mathrm{~nm}$ (diameter) \\
& & $10-14 \mu \mathrm{m}$ (length) \\
Haversian canal & cylindrical & $25-50 \mu \mathrm{m}$ (diameter) \\
& & $1-2 \mathrm{~mm}($ length) \\
Volkman canal & cylindrical & $25-50 \mu \mathrm{m}$ (diameter) \\
& & $200-500 \mu \mathrm{m}$ (length) \\
\hline
\end{tabular}

In piezoelectric materials deformation causes separation of charges within which result in electrical dipoles. Dipoles are quantified in terms of polarization vector. Polarized bodies subsequently experience surface polarization of charge. Korostoff's (1977, 1979, 1981) main hypothesis was that the parallel arrangements of collagen molecules in lamellae of bone would result in layers with alternating polarization vectors. He argued that this would lead to an unstable configuration and subsequent loading would lead to reversals of those vectors resulting in piezoelectric effects that would explain experimental data.

Today it is widely accepted that the physiologically significant SGPs in bone are not caused by piezoelectricity but by the second phenomenon known as electrokinetics or streaming potentials (Anderson and Eriksson, 1970). Streaming potentials occur when ionized liquids are forced to flow through channels inside a solid that has a charged surface. Positively/negatively charged ions are attracted to the negatively/positively charged solid surfaces creating a positively/negatively charged stationary liquid layer 
alternatively called the hydrodynamic slip plane. These planes cause similarly charged (as the layer) free ions to flow inside the channel with higher velocities and the flow results in net potential difference. The key differences between SGPs caused by those theories is that in case of streaming potentials SGPs will last as long as there is a flow whereas SGPs due to piezoelectric effects will persist as long as there is a deformation.

Although there has been an enormous progress in understanding the mechanotransduction in bone one of the key aspects of it, which is the understanding of the process of transmission of information between mechanosensory osteocytes and bone-forming osteoblasts is yet to be done.

\subsection{Material Models for Bone}

The essential idea of composite modeling of bone is to predict the overall mechanical properties, given knowledge of the mechanical properties, relative amounts, shapes, and microstructural arrangements of the individual phases of which it is composed. There are several broad generic classes of composite materials, which differ from one another regarding the manner in which their phases are spatially distributed. In all of them, there is a continuous matrix phase. They differ regarding the characteristics of the second phase, which is distributed within the matrix. When the material of the second phase is in the form of equiaxed particles, the resulting structure is known as a "particulate composite". In a "chopped" fiber composite, the length of the fiber is relatively short. A fiber composite may be "unidirectional" if all the fibers are aligned, or "random" if there is no preferred orientation. In a "laminate" unidirectional fiber composite layers are arranged in a controlled periodic manner.

\subsubsection{Particulate Composite Models}

Currey (1964) was the first to apply composite theory to bone discussing bone as a two-phased material governed by the "law of mixtures", alternatively known as Voigt (1929) or "isostrain" model.

$$
E_{b}=V_{o} E_{o}+V_{m} E_{m}
$$

where subscripts $b, o$ and $m$ stand for bone, organic and mineral respectively.

Katz (1971) and Piekarski (1973) utilized the Reuss (1929) or "isostress" model: 


$$
\frac{1}{E_{b}}=\frac{V_{o}}{E_{o}}+\frac{V_{m}}{E_{m}}
$$

Shortcomings of these models were that tensile stresses in the mineral phase calculated using the first model far exceeded that which could be expected to be borne by ceramic material without fracturing, and the second model correctly predicted overall stiffness leading to the paradoxical conclusion that elastic properties of HA are not important.

Table 1.2. Material properties of the major components of bone

\begin{tabular}{llll}
\hline Bone component & Strength & Stiffness & ultimate strain \\
\hline Hydroxyapatite & $0.1 \mathrm{GPa}$ & $130 \mathrm{GPa}$ & 0.001 \\
Collagen at 0.001 stain & $1 \mathrm{MPa}$ & $50 \mathrm{MPa}$ & \\
\hline
\end{tabular}

Subsequently, a model was proposed involving a combination of the "isostress" and "isostrain" theories (Hirsch, 1962; Dougdill, 1962, Piekarski, 1973):

$$
\frac{1}{E_{b}}=x\left[\frac{1}{V_{o} E_{o}+V_{m} E_{m}}\right]+(1-x)\left[\frac{V_{o}}{E_{o}}+\frac{V_{m}}{E_{m}}\right]
$$

with $\mathrm{x}$ and (1-x) being the relative proportions of the material behaving according to the upper and lower bounds, respectively. Piekarski (1973) estimated x to be 0.925 for bone. Later, more rigorous composite theory approach of Hashin and Shtrikman (1963) based on principles of variational calculus was utilized for production of more closely spaced upper and lower bounds of Young's modulus of bone.

In summary particulate models ignored the actual microstructure and porosity, therefore, leading to large gaps between bounds.

\subsubsection{Fiber Composite Theories}

Currey (1969a) pioneered the approach of modeling bone with actual consideration of its microstructure to explain the steep rise in predicted stiffness of bone with a moderate increase in bone mineral content (1969b). In this approach, the organic 
phase was considered to be the matrix and the HA crystallites to be reinforcing fibers, with a major assumption that all HA crystallites are parallel to the axis along which stress is applied.

$$
E_{b}=E_{o} V_{o}+E_{m} V_{m}\left(1-\frac{2}{\beta L} \tanh \left[\frac{\beta L}{2}\right]\right)
$$

where

$$
\beta=\left[\frac{2 \pi G_{o}}{E_{m} \Lambda_{m} \ln \left(a / a_{m}\right)}\right]
$$

and where $L$ is the length of the HA needle, $G_{0}$ is the shear modulus of the organic matrix, $A_{m}$ is the cross-sectional area of an individual HA crystallite, $a_{m}$ is the radius of the HA crystallite and $a$ is the mean separation distance between the crystallites. The anisotropic properties were obtained via tensor transformation laws for a transversely isotropic (TI) material. The major shortcoming of this model was that in reality HA crystals are not unidirectional.

\subsubsection{Osteonal Modeling}

Katz (1971) in a series of papers proposed to approach the problem from a higher length scale, namely treating osteons as fibers reinforcing the surrounding matrix. The matrix phase included cement lines and interstitial lamellae. The model was a two-tiered, hierarchical composite model. It starts with the calculation of Young's modulus of a single osteon using:

$$
E_{b}=E_{c} V_{c} \frac{\left(1-v_{c} \nu_{b}\right)}{\left(1-v_{c}^{2}\right)}+\sum_{n} E_{m} V_{m} g\left(\phi_{n}\right)\left(\cos ^{2} \phi_{n}-v_{b} \cos ^{2} \phi_{n} \sin ^{2} \phi_{n}\right)
$$

where $v_{o}$ and $v_{b}$ are the Poisson's ratios of the organic phase and bone, respectively, and $g(\phi)$ is the distribution function of the crystal orientations. This expression was derived based on Krenchel's (1964) formulation, with the first part of the equation referring to collagen assuming no dependence on direction and the second part providing the effect of HA. The next assumption was that osteons are arranged in pseudo-hexagonal packing and the Hashin and Rosen (1964) formulation was applied to provide a set of bounds for five 
independent effective elastic constants required for complete characterization of anisotropic elastic properties.

In summary, this and previously discussed models are all based on logical, theoretically predicted assumptions on various properties of aggregate phases without strong experimental support. The majority of investigators therefore, have chosen to investigate a "material" which was in one level below the whole bone, a method successfully used by engineers for complex materials without a severe degree of anisotropy. Namely, using standardized geometry of specimens and applications of statistical methods to study rheological properties of materials.

\subsection{Elastic Properties of Bone}

Numerous studies of elastic properties of human cortical bone have demonstrated that they depend on bone type (femur or tibia), bone cortex (anterior, posterior, lateral or medial) and anatomical location (distal, proximal). This behavior is unlike that of steel, aluminum and most plastics, but is similar to wood. Tensorial elastic moduli of bone, determined ultrasonically and with conventional loading-deformation (stress-strain) studies using elastic symmetry based on inherent structural symmetry of bone led to resemblance of that of transversely isotropic material. The plane of symmetry is perpendicular to the longitudinal axis of long bones. A summary of properties obtained from various studies is given in Table 1.3. 
Table 1.3. Tensorial and technical elastic moduli of human cortical bone.

\begin{tabular}{|c|c|c|c|}
\hline Bone Type & $\begin{array}{c}\text { Tensorial Moduli } \\
(\mathrm{GPa})\end{array}$ & Engineering Moduli & Reference \\
\hline \multirow{5}{*}{ Dry Human Femur } & $\mathrm{C}_{1111}=23.4$ & & \multirow{5}{*}{$\begin{array}{l}\text { Yoon and Katz } \\
\text { (1976) }\end{array}$} \\
\hline & $\mathrm{C}_{1122}=9.06$ & & \\
\hline & $C_{1133}=9.11$ & & \\
\hline & $\mathrm{C}_{3333}=32.0$ & & \\
\hline & $\mathrm{C}_{2323}=5.4$ & & \\
\hline \multirow{12}{*}{ Wet Human Femur } & & $\mathrm{E}_{\text {long }}=17 \mathrm{GPa}$ & \multirow{12}{*}{$\begin{array}{l}\text { Reilly and Burstein } \\
\qquad \text { (1975) }\end{array}$} \\
\hline & & $\mathrm{E}_{\text {trans }}=11.5 \mathrm{GPa}$ & \\
\hline & & $\mathrm{E}_{\text {trans }}=11.5 \mathrm{GPa}$ & \\
\hline & & $\mathrm{G}_{\text {long }}=3.6 \mathrm{GPa}$ & \\
\hline & & $\mathrm{G}_{\text {trans }}=3.3 \mathrm{GPa}$ & \\
\hline & & $\mathrm{G}_{\text {trans }}=3.3 \mathrm{GPa}$ & \\
\hline & & $v_{\text {long }}=0.58$ & \\
\hline & & $v_{\text {trans }}=0.31$ & \\
\hline & & $v_{\text {trans }}=0.31$ & \\
\hline & & $\sigma^{\text {ult }}=148 \mathrm{MPa}($ long $)$ & \\
\hline & & $\sigma^{\mathrm{ult}}=49 \mathrm{MPa}($ trans $)$ & \\
\hline & & $\rho=2.0\left(\mathrm{~g} / \mathrm{cm}^{3}\right)$ & \\
\hline
\end{tabular}




\subsection{Viscoelasticity of Bone}

Most structural materials are nearly linearly elastic under small strain as they retain linearity between load and deflection. Viscoelastic materials retain linearity between load and deflection, but the linear relationship depends on a third parameter, time. For this class of materials, the present state of deformation cannot be determined completely unless the entire loading history is known. A linear elastic solid may be said to have a simple memory: it remembers only one configuration, namely the unstrained neutral state of the body. Viscoelastic materials do not behave this way; they remember the past. The load-deflection relationship of a viscoelastic material is given by means of a convolution integral. Convolution of two functions is mathematically defined as (Lathi, 1992):

$$
C(t)=f(t) * g(t)=\int_{0}^{t} f(\xi) g(t-\xi) d \xi
$$

Some characteristic phenomena in viscoelastic materials are (i) if the stress is held constant, the strain increases with time (creep); (ii) if the strain is held constant, the stress decreases with time (relaxation); (iii) the effective stiffness depends on the rate of application of the load; (iv) if cyclic loading is applied, hysteresis (a phase lag) occurs, leading to a dissipation of mechanical energy; (v) acoustic waves experience attenuation; (vi) rebound of an object following an impact is less than 100\%; and (vii) during rolling, frictional resistance occurs.

Rauber (1876) was the first to demonstrate viscoelasticity in bone by studying creep as well as anisotropy and strength of bone. Later studies of viscoelasic behavior of bone include studies by Smith and Keiper (1965), Sedlin (1965), Knets and Vilks (1975), Melnis and Laizan (1978) and many other authors.

Lakes and Katz (1974) applied the Boltzman superposition integral

$$
\sigma_{i j}(t)=\int_{-\infty}^{t} C_{i j k l}(t-\tau) \frac{d \varepsilon_{k l}(\tau)}{d \tau} d \tau
$$

as a constitutive equation for describing viscoelastic behavior of bone in compression and concluded that it does not apply to bone for the entire frequency range. They extended this work in a series of papers for torsional and biaxial loading conditions (Lakes et al., 
1979; Lakes and Katz, 1979) concluding that non-linear viscoelastic constitutive equations are the best description of nonlinear effects observed experimentally.

The complex hierarchical structure of bone can cause viscoelastic behavior rising from various processes occurring at various length scales. Sasaki et al. (1993) argued that collagen being proteinaceous could give rise to significant viscoelastic behavior. They observed that the relaxation process of bone is a sum of two processes with the first being a simple exponential decay (Debye decay) and the second one following KohrauschWilliams-Watts function (KWW) (Williams and Watts, 1970). Based on the fact that dislocations may be considered as defects responsible for KWW type relaxation for crystalline materials and the assumption that disorder in the collagen structure could be considered as a defect, they speculated that collagen may be responsible for relaxation behavior.

Earlier studies of Lakes and Saha (1979) have demonstrated that at a grosser scale, many interfaces such as cement lines and boundaries between lamellae could be responsible for viscoelastic behavior. By scribing a line on a bone specimen and loading in shear for a long time they demonstrated that the most irreversible strains take place at the cement lines.

With regard to the mineral phase of bone, thermoelastic coupling, which is a result of damping from stress-induced heat flow from the material to its environment or the heat flow in between different heterogenieties in the material, could also be a causal mechanism of viscoelastic behavior. This piezoelectric coupling however was shown to have a negligible contribution (Lakes and Katz, 1979ab). Finally, and very importantly, viscoelasticity in bone could be the result of fluid flow in a porous medium (Biot, 1941).

\subsection{Bone Poroelasticity}

Poroelasticity models the interaction of deformation and fluid in a fluid-saturated porous medium. The deformation of the medium influences the flow of the fluid and vice versa. Biot (1941) generalized the theory of consolidation, developed by von Terzaghi, by extending it to the three-dimensional case and by establishing equations valid for any arbitrary load varied with time. The theory was widely applied to problems of rock 
mechanics. Human bone is a fluid-saturated porous medium and Biot's effective medium theory has been applied to bone by many authors in past decades (Cowin, 1999).

\subsubsection{Structural interfaces of bone}

On the outer surface (periosteum) bone has a specialized, relatively impermeable membrane consisting of an outer layer of dense collageneous fibers and fibroblasts and is reported to serve as a barrier to bone fluid (Li et al., 1987). The inner layer of periosteum together with all the other surfaces that contain vasculature (Havesian and Volkman canals and endosteum) forms a single cellular interface (bone lining cells) (Figure 1.4). These cells are arranged in tight junctions but nevertheless allow fluid transport across this confluent cell layer (Miller and Jee, 1992; Neuman, 1982; Hillsley and Frangos, 1996; Zhang et al., 1998).

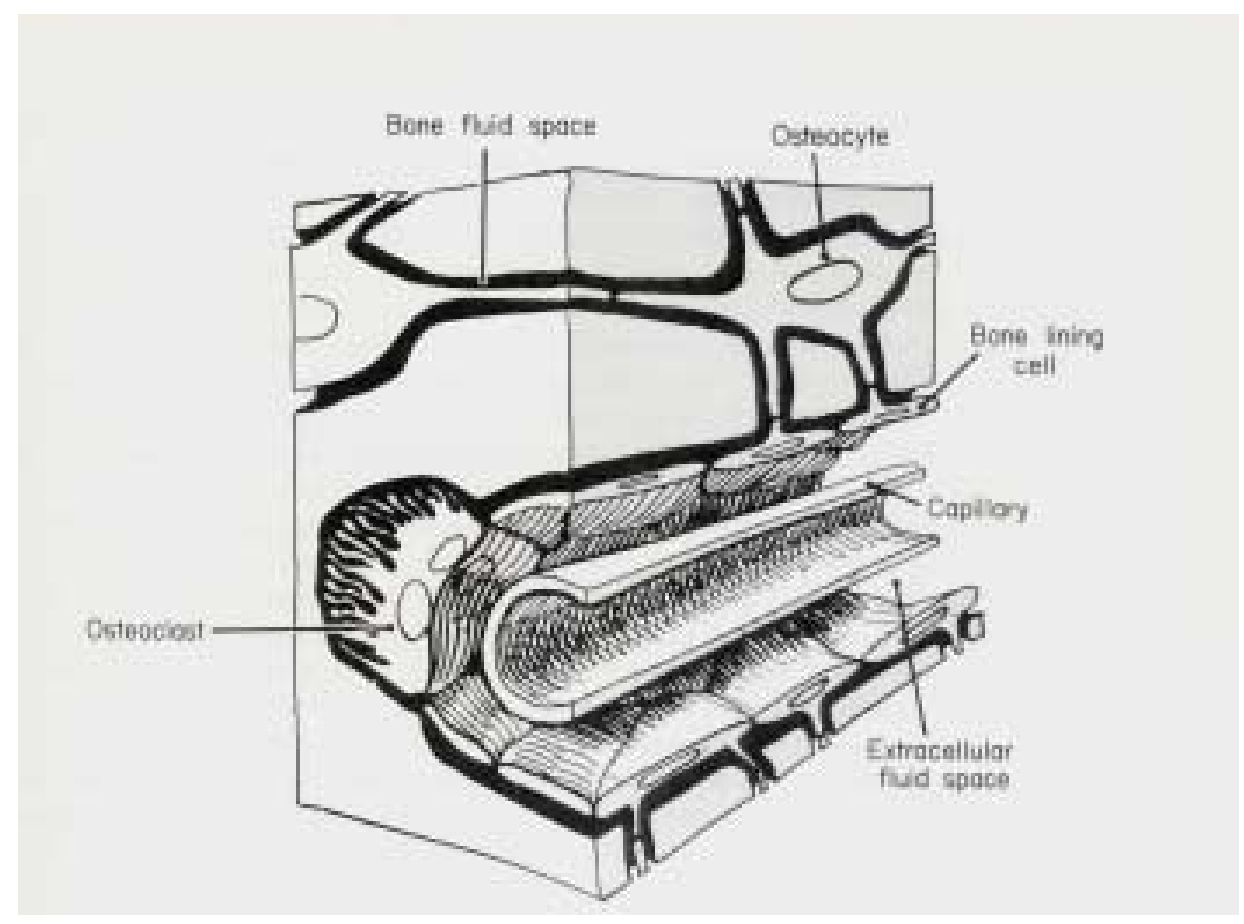

Figure 1.4: Bone lining cells and osteocytes within fluid saturated bone matrix 
Within the lacunae and canaliculi, osteocites and their processes are divided from mineralized matrix by the lacunae-canalicular interface. Evidence from studies using small molecules as tracers suggests that this interface is relatively impermeable (Doty and Schofield, 1992; Knothe Tate et al., 1998; Morris, et al., 1982; Tanaka and Sakano, 1985). It was also shown that as bone matures the permeability across this interface changes.

\subsubsection{Porosities of Bone}

Bone porosities are associated with bone fluids. The fluid in the spaces outside of blood vessels and nerves is called bone fluid, and the fluid in the extracellular space of lacunae and canaliculi is called extracellular fluid (Neuman and Neuman, 1958). The porosities include: 1) vascular porosity, that includes semi-cylindrical passageways of Volkman and Haversian canals and is the largest porosity by its criterion; 2) lacunarcanalicular porosity, that includes lacunae-canalicular space and 3) collagen-apatite porosity, that includes the spaces between the collagen and the crystallites of the HA and is the lowest porosity.

The movement of bone fluid in the collagen-apatite porosity is bound by the interaction with its ionic crystal (Neuman and Neuman, 1958) and therefore is considered to be part of the collagen apatite structure. Based on this fact this level of porosity has been widely neglected in the majority of applications. Most poroelastic models were single (Nominski and Davis, 1970) and two-porosity models (Zhang, et al., 1998).

The key contributions of those models were the description of fluid motion in bone as a result of various load-induced deformations and description of piezoelectric effects. In particular, it was shown that 1) bone fluid in lacunar-canalicular porosity can sustain high pressures for long times and 2) characteristically low pressured (blood pressure) vascular porosity can serve as a reservoir for fluid interchange with lacunarcanalicular spaces. These were key steps in understanding the mechanotransduction in bone. Weinbaum et al. (1994) and Cowin et al. (1995) suggest that lacunae-canalicular space must be filled with glycocalix (a gel like structure) in order to explain slower relaxation times of fluid pressure inside this porosity. 
Nevertheless, important physiological explanations are yet to be given. Considering the fact that bone serves as a calcium and phosphorus reservoir, it is important to reveal how, if at all, those minerals cross the lacunae-canalicular interface. Are the osteocytes involved in the delivery processes of those minerals to bone lining cells, and if they do, how does this process occur? The search of the exact process of mineral retrieval and redeposition lies at the root of many studies (Martin, 1994; McCarthy and Yang, 1992; Soares et al., 1992).

\subsection{Fracture Mechanics of Bone}

Detailed characterization of bone as a material requires understanding of its failure characteristics. The fracture of cortical bone is a complex process that has received considerable attention over the past few decades.

Evans (1957) pioneered the approach of studying the influences of loading conditions, temperature and microstructural details on ultimate strength of bone. Based on these and subsequent studies it was observed that bone behaves like a brittle solid with short total strains (up to 3.5\%) and relatively short post-yield deformation. However, information gained from the studies using traditional strength of materials approach did not shed much light on the actual failure processes in bone. The shortcomings of this approach were appreciated by Evans leading to the conclusion that the solution is to increase the number of specimens studied to ensure statistical significance of results. Bonfield and $\mathrm{Li}$ (1966) outlined that the presence of random surface flaws as a result of specimen preparation would affect the energy to fracture and could be a possible reason in the scatter of the data. They demonstrated that the introduction of a surface crack significantly reduced the energy absorbed during fracture of bone specimens for both longitudinal and transverse directions. Subsequent studies by Piekarski (1970) and Pope and Outwater (1972) have demonstrated that fracture energy reduces as the strain rates increase. However, the nature of these test methods precluded determination of the basic fracture mechanics parameters.

These conclusions have led to utilization of the concepts of linear elastic fracture mechanics (LEFM) for more precise evaluation of the fracture of bone in terms of its 
fracture toughness. Namely, determination of the critical stress intensity factor $\left(\mathrm{K}_{\mathrm{C}}\right)$ and the critical strain energy release rate $\left(\mathrm{G}_{\mathrm{C}}\right)$ of bone.

The two types of specimens that have been proven to be useful for studying bone fracture are the single edge notched (SEN) and the compact tension (CT) specimens. Melvin and Evans (1973) were the first to publish a study applying fracture mechanics concepts. They utilized SEN geometry specimens for studying crack propagation both in longitudinal and transverse directions and reported values for $K_{C}$ and $G_{C}$ for bovine compact bone as a function of various loading rates. In a subsequent study Bonfield and Datta (1976) used SEN specimens to investigate the dependence of fracture stress on the length and radius of curvature of the introduced crack for transverse fracture and concluded that different crack lengths were associated with different fracture stresses but had common fracture toughness.

These studies using SEN specimens did not allow gaining deeper insight into the mechanisms of fracture; i.e. differentiating between the nucleation and propagation of a crack and determining the interaction of a crack front with microstructural features. For this type of study CT specimen geometry is more adequate. Wright and Hayes (1977) utilized CT specimens and studied the effects of bone density and specimen thickness on $K_{C}$ and $G_{C}$ and reported a decrease in $K_{C}$ and $G_{C}$ with a decrease in bone density. Bonfield et al. (1978) conducted a similar study where they investigated the influence of crack velocity on fracture mechanics properties and concluded that the velocity of crack propagation depends on the crosshead speed (loading rate). Hence, the concept of a critical crack velocity associated with a maximum in the fracture toughness of bone was established and shown to represent a transition from controlled to catastrophic propagation (Behiri and Bonfield, 1980; 1984).

It is known that with aging bones becomes more porous and loose mass. High porosity and decreased mass have an obvious influence on bone density. Therefore, the fact that those studies were able to address the issue of describing fracture parameters as a function of density variations was obviously biologically significant.

The majority of studies investigating fracture mechanics of bone have been conducted using bovine cortical bone. Human cortical bone has a distinct microstructural differences compared to bovine cortical bone therefore fracture toughness of human 
compact bone was less well defined (Norman et al., 1991). Norman et al. in a series of papers investigated fracture toughness properties of human cortical bone (Table 1.4).

Table 1.4. Summary of fracture toughness values for human cortical bone (Norman et al., 1995)

\begin{tabular}{lllll}
\hline Bone & $\mathrm{K}_{\text {IC }}\left(\mathrm{MN} / \mathrm{m}^{3 / 2}\right)$ & $\mathrm{K}_{\text {IIC }}\left(\mathrm{MN} / \mathrm{m}^{3 / 2}\right)$ & $\mathrm{G}_{\mathrm{IC}}(\mathrm{N} / \mathrm{m})$ & $\mathrm{G}_{\text {IIC }}(\mathrm{N} / \mathrm{m})$ \\
\hline Tibia & $2.12 \pm 1.32$ & $8.32 \pm 6.44$ & $339 \pm 132$ & $4200 \pm 2516$
\end{tabular}

*Mean \pm S.D.

It was demonstrated that despite the significant weakness of the strength of human bone compared to bovine bone the toughnesses were similar (Norman et al., 1995). It was also demonstrated that fracture toughness in shear was approximately 13 times higher than in tension (Norman et al., 1996). This was consistent with the behavior of fiber reinforced composite materials. Recent studies have demonstrated that among the morphological constituents of bone structure, the porosity and osteonal density were the best explanatory mechanisms of toughness variations (Yeni et al., 1997). It was also demonstrated that water content, apparent density were the best explanatory mechanisms of toughness variations as a function aggregate phases (Yeni et al., 1999).

\subsection{Summary}

In summary, in this chapter a general introduction was given about physiological functions of bone as a tissue and properties of bone as a material. Also, the fundamental directions of bone research have been outlined with a brief summary of results. It is clear that in order to better understand the physiology of bone detailed knowledge is necessary about its material properties. As it was discussed in section 1.6., despite the tremendous amount of research done in development of a material model unique for bone, the resultant models had significant shortcomings in predicting bulk material properties based on material properties of the constituent phases. As a result bone researchers were forced to take alternative approaches in order to understand this material. Generally 
accepting the results of material models researchers concluded that bone could be considered as an orthotropic (transversely isotropic in some applications) material and proceeded in studying the thermorheological characteristics of this material and studied its failure behavior. In today's bone literature majority of researchers use the results obtained by Burstein and co-workers considering bone as a linear elastic material in the physiological strain range and undergoing plastic deformation at elevated strain levels. Perhaps, those assumptions are satisfactory for various applications but in principle this leads to an inaccurate representation of this material. Research conducted by various authors during past five decades demonstrates that these assumptions are in fact not accurate. Lakes and co-workers in 1970s published series of papers demonstrating that bone is not an elastic material instead it has a strong time dependence. They continued their work for the last three decades and were able to identify various structural constituents of bone's microstructure giving rise to this behavior. Time dependent nature of this material is somewhat obvious considering the fact that it is saturated with bodily fluids, hence deformations induced on bone cause fluids to move which in turn would guide the deformation of the solid matrix. The interdependence of those two behaviors demonstrates possibility of the involvement of time. This is extremely crucial in accurate characterization of the damage development and failure of this material, which is the main objective of this dissertation work.

\subsection{The Overall Objectives of This Dissertation Work}

Characterization of damage behavior is one of the fundamental problems of solid mechanics. Understanding damage in bone has an extra advantage, because it could shed a considerable amount of light in tackling one of the key issues of current bone reaserch, which is to understand the mechanisms of bone fragility. Hence, in long term it could lead to increased knowledge about debilitating bone diseases such as osteoporosis and help reveal the mechanisms behind the stress fractures, which are significant orthopedic concerns today.

There has been considerable amount of research done in understanding the microcracking behavior in bone. One of the key issues that has not been resolved is how and where those cracks initiate in bone's microstructure. The first objective of this 
research is to design an experimental protocol to study damage genesis in bone and investigate the influence of early damage development, called submicroscopic diffuse damage, on fracture toughness. To investigate damage development under combined tensile, compression and shear loading the second objective was to study damage accumulation and failure behavior of bone under 3-point bending. And finally develop a constitutive formulation for this material that includes damage. The theoretical framework, which would be utilized for the achievement of this goal would be the Continuum Damage Mechanics based on the Thermodynamics of Irreversible Processes. 


\section{CHAPTER 2}

INITIAL STAGES OF DAMAGE IN HUMAN COMPACT BONE (Parsamian, G.P. et al., 2000. $46^{\text {th }}$ Ann. Meet. ORS; Parsamian, G.P. and Norman, T.L., 2001. J. Mat. Sci: Mat. Med)

\subsection{Introduction}

It was already mentioned that throughout life bone constantly remodels by absorbing old bone and replacing with new bone. This process leads to the formation of secondary osteons. Tomes and De Morgan (1853) were the first to describe this phenomenon. It was not until recently when more detailed accounts by Frost (1963a,b), Johnson (1964, 1966) and Lacroix (1974) were able to characterize this "tunneling" and "refilling" processes in bone. Frost (1963a) proposed that this process was carried out by groups of cells functioning as organized units called basic multicellular units (BMUs). The BMU remodeling sequence follows well-defined phases beginning with activation (A), followed by resorption (R) then followed by formation (F). This process is commonly referred as the A-R-F sequence of bone remodeling. BMUs operate on bone periosteal, endosteal, and trabecular surfaces as well as within cortical bone. Histomorphometric data for osteonal BMUs is given in Table 2.1.

Various activation signals are thought to be present in the skeleton, including chemical and mechanical or electrical, but just how or where they affect cells, and what sorts of intermediate messengers may exist are questions still unanswered.

Rodan and co-workers (1975) observed that the osteoblasts-turned-lining cells process receptors for hormones and vitamins (PTH and vitamin D) whereas osteoclasts do not. Furthermore they observed that bone cells respond to those signals in the ways that are consistent with osteoclast activation thus hypothesized that bone-lining cells are responsible for initiating BMUs. Other authors (Frost, 1966; Tschantz and Retishauser, 1967; Chamay, 1970; Swanson et al., 1971) have suggested that osteonal BMUs are activated by mechanical loads or fatigue damage or both. Osteonal debonding was thought to be the triggering event of bone remodeling. Martin and Burr (1982) proposed a more specific mechanism for the generation of new osteonal BMUs in response to fatigue damage. They argued that osteonal bone is not only designed so that microcracks will be both easily initiated and easily stopped but also it is designed so that microdamage itself 
initiates the reparative remodeling process. They suggested that this happens because debonding of the osteon during crack trapping causes changes in the portion of the Haversian system adjacent to the crack, which initiate the activation of a new osteonal

BMU. These new remodeling units resorb the damaged regions and replace them with new bone (Figure2.2).

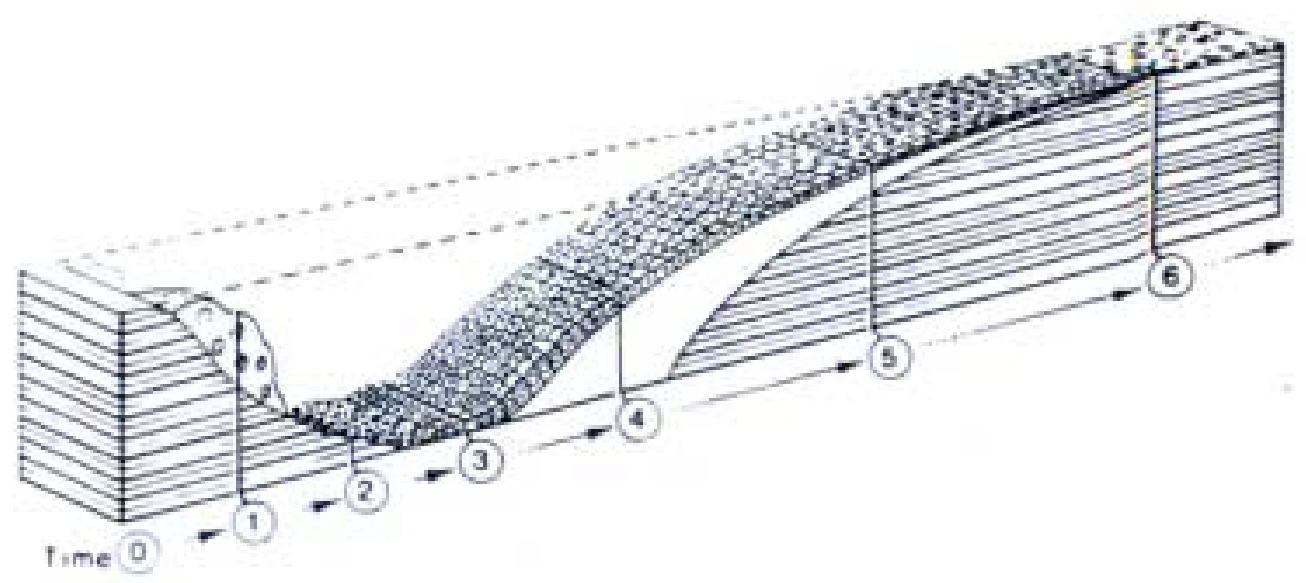

Figure 2.1. Schematic representation of the bone remodeling sequence. The resorption (R) sequence (1 - 3): 1) osteoclastic phase; 2) mononuclear phase; 3) preosteoblastic phase. The Formation (F) sequence (4 - 6): 4) unmineralized matrix (osteoid); 5) mineralization; 6) formation of bone lining cells (final thickness) (Frost, 1963a). 
Table 2.1. Histomorphometric data for osteonal BMUs in human rib (Frost, 1969).

\begin{tabular}{llll}
\hline & & \multicolumn{2}{c}{ Age (yrs) } \\
\cline { 2 - 4 } & $1-9$ & $30-59$ & $70-89$ \\
\hline $\mathrm{A}_{\mathrm{f}}\left(\# / \mathrm{mm}^{2}\right)$ & $2.7 \pm 1.0$ & $0.21 \pm 0.54$ & $0.66 \pm 0.5$ \\
$\mathrm{~A}_{\mathrm{r}}\left(\# / \mathrm{mm}^{2}\right)$ & $2.0 \pm 0.62$ & $0.25 \pm 0.33$ & $0.84 \pm 0.46$ \\
Sigma F (days) & $51 \pm 47$ & $73 \pm 58$ & $109 \pm 55$ \\
$\mathrm{f}_{\mathrm{a}}\left(\# / \mathrm{yr} / \mathrm{mm}^{2}\right)$ & $19 \pm 9$ & $1.1 \pm 0.5$ & $2.2 \pm 1.1$ \\
$\mathrm{~V}_{\mathrm{f}}(\% / \mathrm{yr})$ & $38 \pm 24$ & $1.8 \pm 0.6$ & $4.4 \pm 1.5$ \\
\hline
\end{tabular}

$\mathrm{A}_{\mathrm{f}}=\#$ of refilling osteons $/ \mathrm{mm}^{2}$

$\mathrm{A}_{\mathrm{r}}=\#$ of resorbing osteons $/ \mathrm{mm}^{2}$

Sigma $\mathrm{F}=$ refilling period

$\mathrm{f}_{\mathrm{a}}=$ activation frequency

$\mathrm{V}_{\mathrm{f}}=$ bone formation rate 


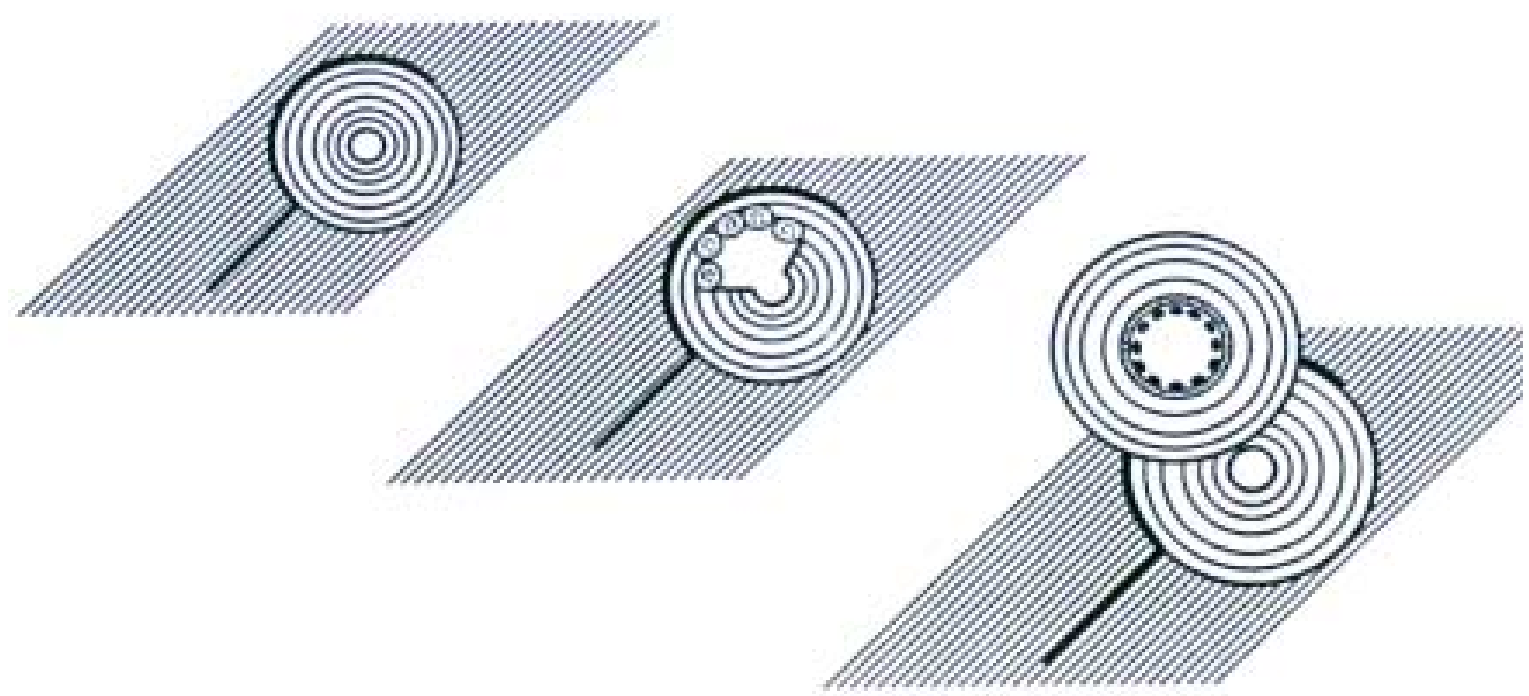

Figure 2.2. Schematic diagram showing how osteonal debonding may initiate BMU activation. Crack around the cement line creates a disuse state, which leads to bone absorption, followed by bone deposition. As a result crack could be repaired by the newly formed osteon.

\subsubsection{Fatigue of Bone}

Recent increases in athletic activities have led to the increased number of fatigue fractures of bones. Fatigue fracture, clinically known as a stress fracture, is an overuse injury to bone that results from the damage accumulation (Jones at al., 1989). It has been estimated that up to $10 \%$ of all sports-related injuries involve stress fractures (Meyer, 1993; Monteleone, 1995; Smrcine, 1991; Sterling et al., 1992). Such fractures occur in bones of lower limb including metatarsals, calceneus, tibia, fibula, femur and pelvis. The tibia has been reported to surpass metatarsals and be the most common location for the development of a stress fracture (Meyer, 1993; Maitra and Johnson, 1997).

Fatigue is defined as the progressive loss of strength and stiffness that occurs prior to failure of materials subjected to repeated loads. These changes have been attributed to the formation and growth of microscopic cracks that develop within the material during each cycle (O’Brien and Reifsnider, 1977).

Evans and Lebow (1957) were the first to perform fatigue testing of bone and to establish S-N curve for bone. Carter and co-workers in a series of papers continued those 
early studies of fatigue behavior of compact bone resulting in the most comprehensive investigations of fatigue behavior of devitalized cortical bone. They have demonstrated that: 1) two-fold decrease in the loading amplitude resulted in a four-fold increase in fatigue life, 2) the change of temperature from the body temperature to the room temperature causes a three-fold increase in the fatigue lifetime and 3) relatively small $(\approx 5 \%)$ increase of bone density, without any microstructural changes, doubles the fatigue lifetime (Carterand Hayes, 1976a; Carter et al., 1976b). In the subsequent studies they have discussed the loss of stiffness and strength with an increase of fatigue loading and suggested that this behavior is similar to engineering materials and is caused by the accumulation of small microcracks at the cement lines and at the interlamellar bands (Carter and Hayes, 1977a,b).

In the later studies Carter et al. (1981a,b) investigated the influence of mean strain range and tissue 's microstructural characteristics on the fatigue behavior and concluded that fatigue life can be effectively correlated with the strain range and that the specimen modulus was the best indicator of the variations in fatigue life. They have predicted that fatigue strength of human cortical bone after 10 million cycles could be $7 \mathrm{MPa}$. These results indicates that bone has an extremely long fatigue life under physiological loading condition, yet stress fractures occur after 100-1000 miles of continuous running and vigorous exercising (Morris and Blickenstaff, 1967; Devas, 1975; Barrow and Saha, 1988; Geyer et al., 1993; John et al., 1994). Based on this argument Schaffler and coworkers performed fatigue testing of human cortical bone specimens under strain control with low strain magnitudes (up to $1500 \mu$ strain) and at a physiological strain rate of $0.03 \mathrm{~s}^{-1}$ and found that it will take about 13 million cycles to fracture the specimens (Schaffler et al., 1989, 1990). They also observed that bone exhibits a rapid 2-12\% loss of its stiffness at the initial stages of fatigue followed by stabilized drop of stiffness until failure after approximately 13 million cycles (Schaffler et al., 1990). These observation lead them to speculate that other phenomena, i.e. 1) brief periods of higher strain, 2) reduced bone mass and increased intracortical porosity due to bone remodeling or 3) preexisting microdamage need to operate in vivo in conjunction with the cyclic loading to cause a fatigue fracture. There have been considerable amount of studies, both animal 
and in vitro addressing those issues (Burr et al., 1985, 1990, 1992, 1995, 1997; Schaffler et al., 1995; Mashiba et al., 2000; Verborght et al., 2000; Bentolila et al., 1998).

\subsubsection{Bone Microdamage}

Evaluating the role that microdamage plays in the bone fragility that is a result of various skeletal disorders requires a reproducible and accurate way to recognize the microdamage that is present. To assess damage in bone researchers have traditionally followed the protocol introduced by Frost (1960) and modified by Burr and Stafford (1990) and Burr and Hooser (1995). This procedure involved en bloc staining of specimens in ascending concentrations of basic fuchsin dye in alcohol followed by cutting approximately $250 \mu \mathrm{m}$ thick slices and observing for damage using a transmitted light microscope (Figure 2.3.). Microcracks are defined as having sharp borders with a halo of increased basic fuchsin stain surrounding them. Cracks that have sharp borders but no halo of increased stain have been considered as preparation artifacts. This protocol was used by various researchers to detect microdamage generated in vivo (Frost, 1960; Mori and Burr, 1990; Norman and Wang, 1997) and in vitro (Forwood and Parker, 1989, Akhter et al., 1993). Lee et al. (1998) proposed an alternative fluorescence-aided technique for the damage assessment of cortical bone using epifluorescence microscopy. Table 2.2 summarizes microdamage data from various studies. 


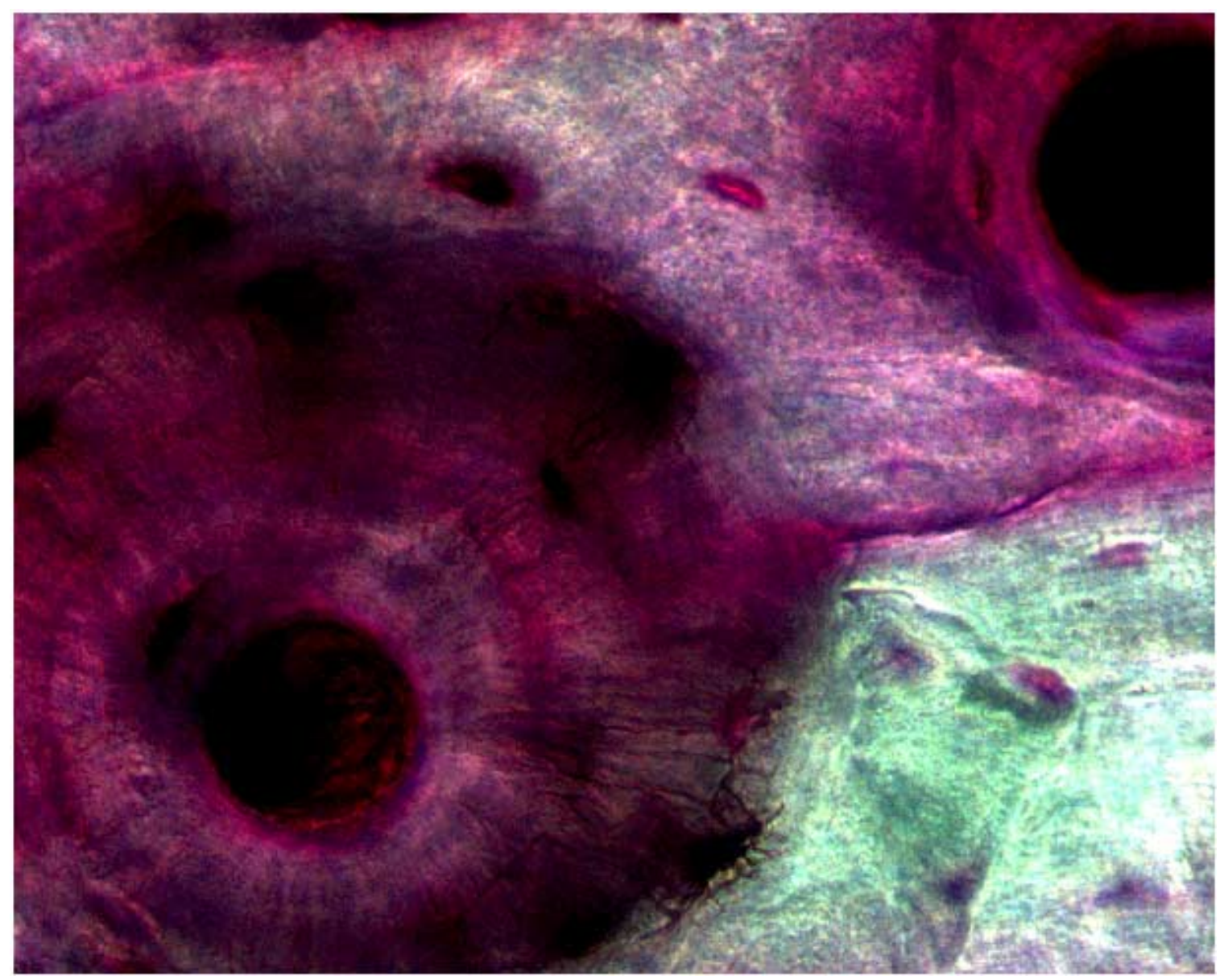

Figure 2.3. An image of transverse section of human cortical bone stained with basic fucsin, demonstrating a typical microcrack in bone matrix.

Damage undoubtedly initiates at tissue levels beyond the resolution of the light microscope. Schaffler and co-workers (1994) developed a new staining technique using lead-uranyl acetate dye and were able to study damage at a finer scale of bone microstructure using back-scattered electron microscopy. They argued that, since the intact bone matrix does not normally stain with the lead-uranyl acetate, the presence of lead-uranyl staining in the regions outside the cracks per se would indicate a change in the matrix permeability to the stain suggesting ultrastructural level disruption of the matrix. They have demonstrated that some of the naturally occurring microcracks visible under light microscopy showed marked extensions of diffuse staining spreading from the crack edges. Those diffusely stained regions have appeared to be consisting of a large number of small microcracks when they were observed using back-scattered electron 
microscope. Those regions were subsequently named as diffuse damage regions. Similar observations of networks of tiny cracks were observed earlier by Zioupos and Currey (1994) using fluorescein stained specimens of bovine plexiform bone under laser scanning confocal microscope (LSCM).

Table 2.2. Microdamage data from various studies.

\begin{tabular}{ccccc}
\hline Bone & Method & $\begin{array}{c}\text { Crack density } \\
\left(\# / \mathrm{cm}^{2}\right)^{*}\end{array}$ & $\begin{array}{c}\text { Crack length } \\
(\mu \mathrm{m})^{*}\end{array}$ & Author \\
\hline Human Rib & TL & 14.25 & $88 \pm 38$ & $\begin{array}{c}\text { Burr and } \\
\text { Stafford (1990) }\end{array}$ \\
Human Rib & FAD & $13.39 \pm 9.31$ & $82.22 \pm 28.95$ & $\begin{array}{c}\text { Lee et a. (1998) } \\
\text { Human Femur }\end{array}$ \\
& TL & $21.45 \pm 15.79$ & N/A & $\begin{array}{c}\text { Norman and } \\
\text { Wang (1997) }\end{array}$ \\
\hline
\end{tabular}

$\mathrm{TL}=$ Transmitted Light Microscopy

$\mathrm{FAD}=$ Fluorescence Aided Detection

$*$ Mean \pm St.Dev.

\subsubsection{Confocal Microscopy}

Confocal microscopy, which was utilized in this study, offers several advantages over conventional light microscopy. The shallow depth of field $(0.5-1.5 \mathrm{~mm})$ of confocal microscopes allows information to be collected from a well-defined optical section rather than from most of the specimen as in conventional light microscopy. Consequently out of focus fluorescence is virtually eliminated, which results in an increase in contrast, clarity and detection.

The essential idea of a LSCM is the substitution of a conventional light source with a monochromatic laser line and the addition of confocal plane, which eliminates out of focus information. In fluorescence mode, the laser light excites the fluorochrome within the specimen causing a chromatic shift such that the wavelength of the emitted 


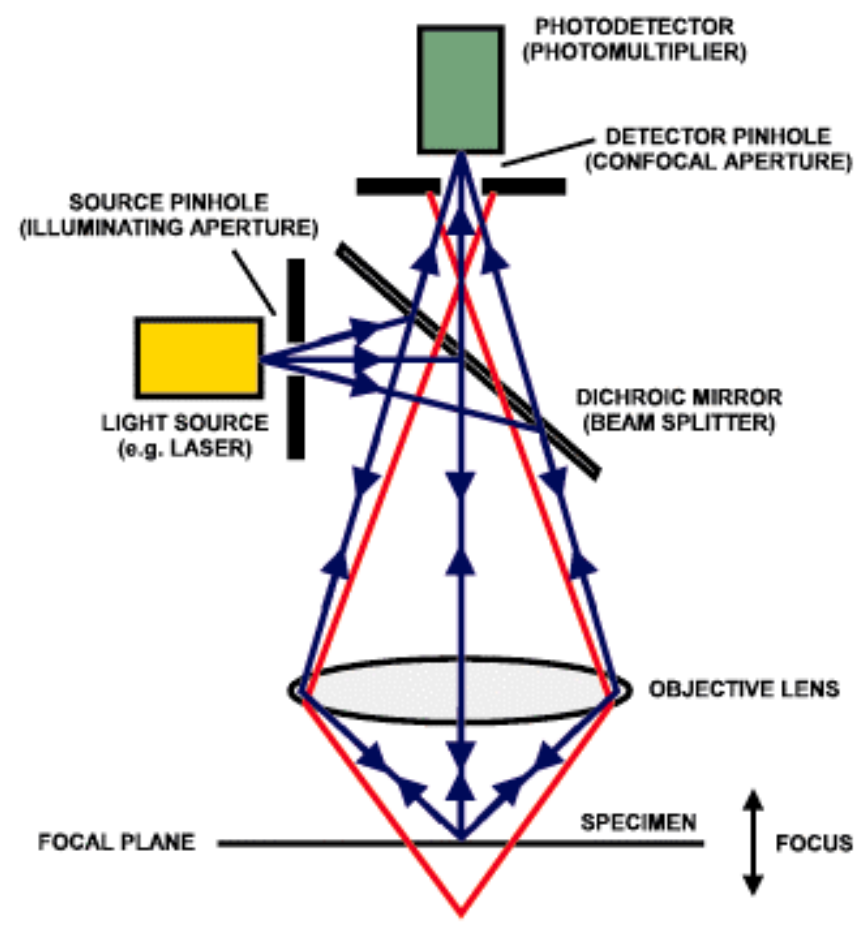

Figure 2.4. Schematic diagram of a typical LSCM system.

fluorescent light is longer then the excitation light. The fluorescent light is then directed back through microscope objective lens and is focused by the achromat lens onto the confocal point, where the confocal aperture allows only light from the focal point to pass through to the detector (Figure 2.4).

\subsubsection{Objectives}

The hypothesis that bone damage begins at the ultrastructural level has only been supported by indirect evidence. The objectives of this portion of the study of damage in bone were to design an experimental technique that would allow us to monitor the initial stages of damaging events in human cortical bone. Specifically: 1) study whether the rapid drop of stiffness at the initial stages of fatigue testing of bone is associated with ultrastructural damage, 2) identify the microarchitectural sites of bone microstructure associated with the origins of damage, 3 ) investigate whether those changes are reflected on macrostructural variables (fracture toughness) and finally 4) speculate whether those 
early damaging events could be responsible in the triggering of remodeling responses and how it could possibly explain the site specificity of bone intracortical remodeling. 


\subsection{Materials and Methods}

\subsubsection{Specimen Preparation}

The specimens were machined from the tibia, which was from a 46-year-old male who had died from a cause unrelated to skeletal conditions. $5 \mathrm{~cm}$ long five diaphyseal segments were cut and cleaned from bone marrow. Anteromedial, anterolateral and posterior regions were subsequently planed using $\mathrm{CNC}$ machining device. After achieving desired planed surfaces the $\mathrm{CNC}$ was programmed to cut the half thickness of the desired specimen geometry (Figure 2.5a). When all three surfaces were machined the section was removed and the three anatomical planes were cut and separated. Subsequently they were placed into the specifically designed fixture and the other surface was planed and the remaining thickness of the specimen geometry was machined. This procedure allowed obtaining a pair of compact tension (CT) specimens from each of the three anatomical planes resulting in 6 specimens from a section (Figure 2.5b). Overall, nineteen CT specimens were machined (Table 2.3). All steps were performed under a constant water irrigation, and those sections that were not immediately machined were wrapped with a $0.9 \%$ saline soaked gaze and were frozen $\left(\right.$ at $\left.-20^{\circ} \mathrm{C}\right)$ in airtight plastic containers to prevent bone degradation. All specimens were polished by use of progressively finer grades of sandpaper and finally polished to a mirror finish with Buehler Micropolish $0.1 \mu \mathrm{m}$ alumina powder. After preparation, all specimens were individually wrapped in $0.9 \%$ saline soaked cloth and were kept in airtight containers at $20^{\circ} \mathrm{C}$ until testing. 


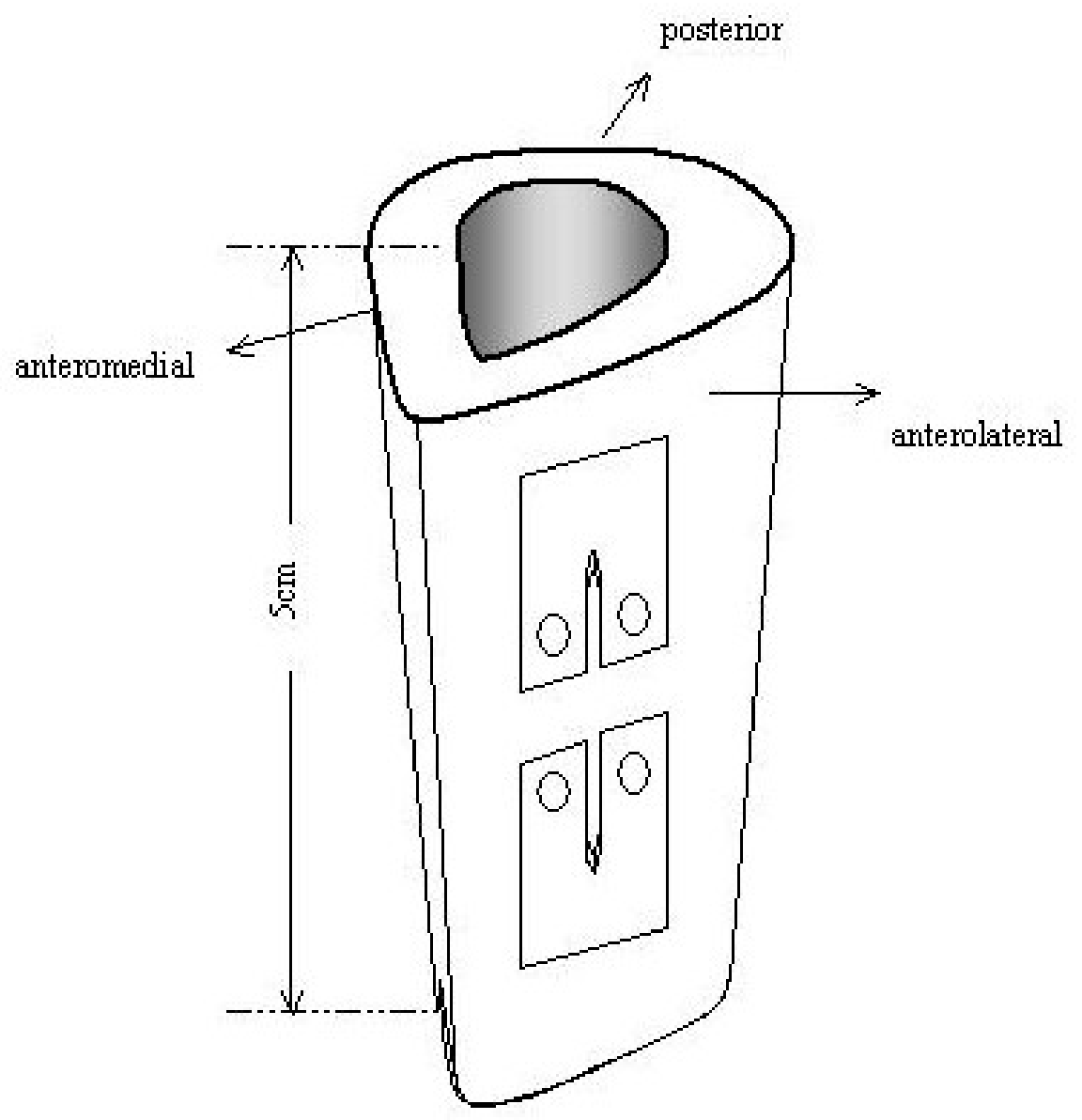

Figure 2.5a. Compact Tension specimens were machined in pairs from anteromedial, anterolateral and posterior cortices of human tibial sections, obtained from distal, medial and proximal locations. 


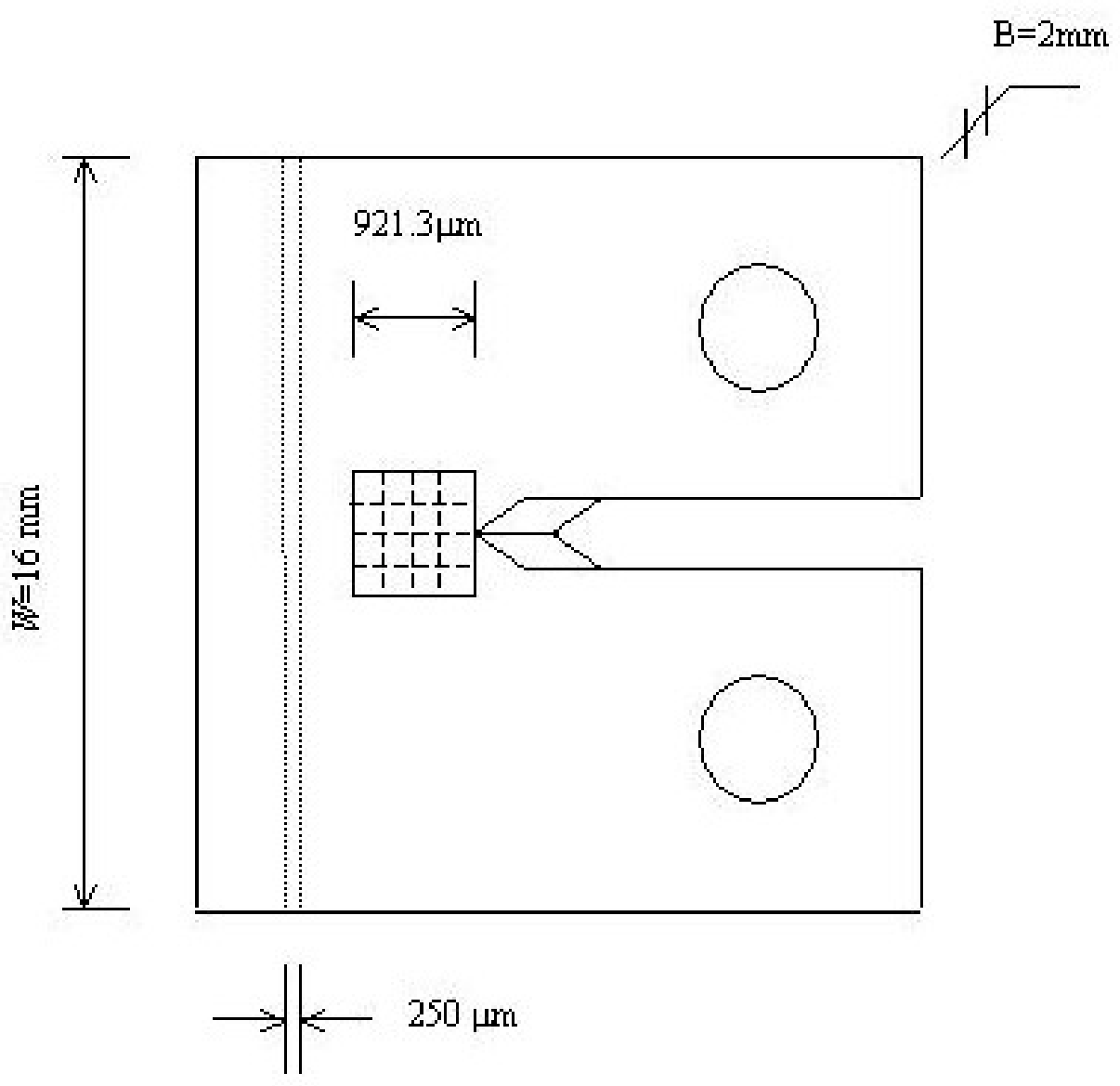

Figure 2.5b. Final geometry of specimens with $B=2 \mathrm{~mm}$ and $a / W=0.5$. An area of $921.3 \times 921.3 \mu \mathrm{m}^{2}$ was investigated for damage development. Objectives with higher magnification allowed further subdivision of this area into 4 and 16 fields for more detailed observations. A $250 \mu \mathrm{m}$ slice, $1 \mathrm{~mm}$ away from the chosen area of damage observations, was cut for morphologic analysis of bone microstructure. 
Table 2.3. A list of specimens and their locations used for this study.

\begin{tabular}{ccc}
\hline & 46 Male, Left Tibia & \\
\hline Specimen Number & Location & Thickness (mm) \\
\hline LT6316p-01 & Distal (poster) & 2.03 \\
LT6316p-02 & Distal (poster) & 2.05 \\
LT6316m-03 & Distal (med) & 2.13 \\
LT6316m-04 & Distal (med) & 2.07 \\
LT6316p-05 & Medial (post) & 2.04 \\
LT6316p-06 & Medial (post) & 2.01 \\
LT6316l-07 & Medial (later) & 1.81 \\
LT6316m-08 & Medial (med) & 1.97 \\
LT6316m-09 & Medial (med) & 1.97 \\
LT6316p-10 & Proximal (post) & 2 \\
LT6316p-11 & Proximal (post) & 2.04 \\
LT6316m-12 & Proximal (med) & 1.94 \\
LT6316m-13 & Proximal (med) & 1.91 \\
LT6316p-14 & Proximal (post) & 1.99 \\
LT6316m-15 & Proximal (med) & 2.04 \\
LT6316l-16 & Distal (later) & 1.87 \\
LT6316l-17 & Distal (later) & 2 \\
\hline
\end{tabular}

Prior to all tests, specimens were stained with $1 \%$ solution of Fluorescein sodium salt (Sigma Chem. Co., St. Louis, MO) dissolved in phosphate-buffered isotonic saline (PBS) (Zioupos et al., 1996) for histological observations using a Zeiss LSM-510 LSCM system equipped with an argon ion laser and attached to a Zeiss Axioplan 2 Fluorescence Microscope. The samples were scanned by the monochromatic $488 \mathrm{~nm}$ laser beam exciting Fluorescein fluorescence and the emission $(520 \mathrm{~nm})$ was recorded by a detector after passing the LP 505 filter.

To minimize the bleaching problem of the stain associated with laser induced quantum mechanical changes in molecules of the stain; the surfaces of specimens were treated with the SlowFade-Light Antifade kit (Molecular Probes, Inc., Eugene, OR). Subsequently specimens were placed on glass slides and were observed for any artifactual cracks and for establishment of baseline in-vivo diffuse damage. A surface scan of an area $\left(921.3 \times 921.3 \mu \mathrm{m}^{2}\right)$ was produced using $10 \times$ objective immediately in front of the crack tip and was chosen for damage measurements (Figure 2.3). 20x and 40x objectives were subsequently used for subdivision of this area into four sections of $460.6 \times 460.6 \mu \mathrm{m}^{2}$ and sixteen sections of $230.2 \times 230.2 \mu \mathrm{m}^{2}$, respectively, for more detailed observations. 
Diffuse damage areas (Df.Dm.) were identified on the $230.2 \times 230.2 \mu \mathrm{m}^{2}$ fields and were circumscribed and measured using LSM 510 image processing software (Figure 2.6). Total Df.Dm. was obtained by summing up the Df.Dm. in all sixteen fields. The Diffuse Damage Density (Df.Dm.D.) parameter was defined as the ratio of total Df.Dm. and Bone Area (B.Ar)(Df.Dm.D.=Df.Dm./B.Ar., $\mathrm{mm}^{2} / \mathrm{mm}^{2}$ ).

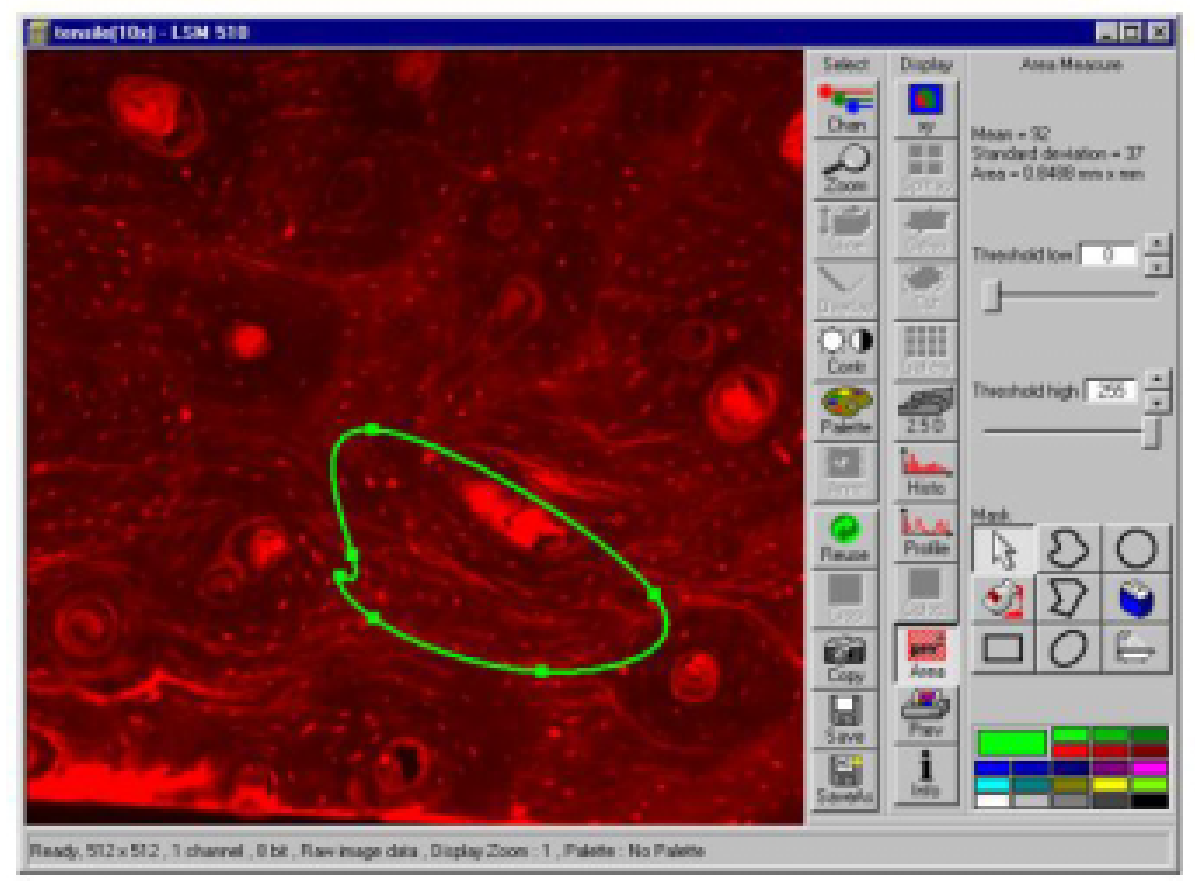

Figure 2.6. An image of a LSM 510 software window with an open image of bone section with a circumscribed diffuse damage area.

\subsubsection{Mechanical Testing}

After establishing the baseline damage, specimens were submerged in a testing bath filled with Fluorescein stain and cycled in tension at a frequency of $2 \mathrm{~Hz}$ on a Materials Testing System (MTS, Minneapolis, MN) servohydraulic testing machine. Five specimens were randomly chosen from various cortices of the tibia for calibration studies. These studies revealed that $12 \mathrm{~N}$ tensile load was an adequate load that was approximately $50 \%$ of the typical load necessary to cause deflection from a linear behavior in monotonic loading. Since the purpose of cycling was to induce various amounts of diffuse damage, specimens were not precracked at the chevron notch and the cyclic load induced crack growth was prevented. The amount of artificially induced 
damage was roughly controlled using the output of the linear variable differential transformer (LVDT) of the MTS machine and by microscopic observations of the surfaces. The residual elongation during cycling was monitored and the cycling was stopped prior to $5-10 \%$ loss of specimen stiffness. To monitor the cumulative damage processes, cycling was periodically stopped after every 100-200 cycles and specimens were examined for generated damage using the method described earlier on the LSCM. Cycling was also stopped after the appearance of first long $(\approx 50-100 \mu \mathrm{m})$ microcracks. These measured damage densities served as the "initial" damage densities for the subsequent toughness tests.

After achieving adequate amounts of damage in each of the specimens, sharp precracks were introduced with razor blade (Norman et al., 1996). Then specimens were submerged back into the testing fixture and were monotonically loaded at a crosshead speed of $2 \mathrm{~mm} / \mathrm{min}$. Load and deformation were acquired and the values of stress intensity factors $\mathrm{K}_{\mathrm{IC}}$ were calculated using

$$
K_{C}=\frac{P_{C}}{B W^{1 / 2}} f\left(\frac{a}{W}\right)
$$

and

$$
f\left(\frac{a}{W}\right)=29.6\left(\frac{a}{W}\right)^{1 / 2}-185.5\left(\frac{a}{W}\right)^{3 / 2}+655.7\left(\frac{a}{W}\right)^{5 / 2}-1017\left(\frac{a}{W}\right)^{1 / 2}+638.9\left(\frac{a}{W}\right)^{9 / 2}
$$

where $B=2 \mathrm{~mm}$ and $\mathrm{a} / W=0.5$

After testing, $250 \mu \mathrm{m}$ thick stained transverse slices were cut $2 \mathrm{~mm}$ ahead of the crack tip (Figure 2.3) of each specimen using a low speed diamond blade metallurgical saw (Buehler, Lake Bluff, IL). Slices were subsequently ground to $150 \mu \mathrm{m}$ thickness and were polished and mounted on glass slides for morphological analysis using Optimas software under a transmitting light microscope at a power of 40× (Martin and Ishida, 1989; Barth et al., 1992). Three adjacent fields, located in the mid section of $2 \times 16 \mathrm{~mm}^{2}$ cross-sections, were examined and Osteon Population Density (OsD) and Porosity (\%P) were measured for all specimens. $\mathrm{OsD}=\#$ of osteons/ Bone Area

Statistical analysis of data was performed using JMP IN ${ }^{\circledR}$ Software (SAS Institute Inc., Cary, NC). Results are reported as means \pm standard deviation. 


\subsection{Results}

Figure 2.7. demonstrates apearence of transverse and longitudinal sections of human cortical bone under a confocal microscope. The fluorescein stain penetrates bone cavities, such as blood channels and lacunae and canaliculi. Subsequent excitation of the stain molecules with a laser beam reveals the location of those molecules of stain and hence highlights the microarchitectural features of bone. During application of loads when microcracks start to appear those newborn cavities immediately get filled with stain and hence appear on confocal micrographs. High signal to noise ratio of confocal microscope ensures the accurate assessment of damage in the ultrastructure of bone.

A nondestructive technique of assessment of damage using intact specimens demonstrated new details of submicroscopic or diffuse damage occurrence. First, histological observations revealed that in the early stages, damaged regions were consistently in the inter-canalicular regions and in the vicinities of lacunae (Figure 2.8). This suggests the possible scenario of damage manifestation as a result of fractures of the mineralized matrix in the inter-canalicular regions. The vacuum regions generated by the process of fracture cause a stain uptake resulting in pooled regions of stain in the vicinities of lacunae (Figure 2.9). Excitation of the Fluorescein molecules reveals the location of damage. The thickness of intact specimens did not allow us to use higher magnification objectives and we were not able to visualize sharp boundaries of the ultrastructural cracks. Therefore, at this level, damaged areas appear blurry and pooled (Figure 2.9). As the defects grew larger, on the order of the lacunae, the boundaries were more evident and the cracks became distinct microscopic cracks (Figures 2.10,2.11). 

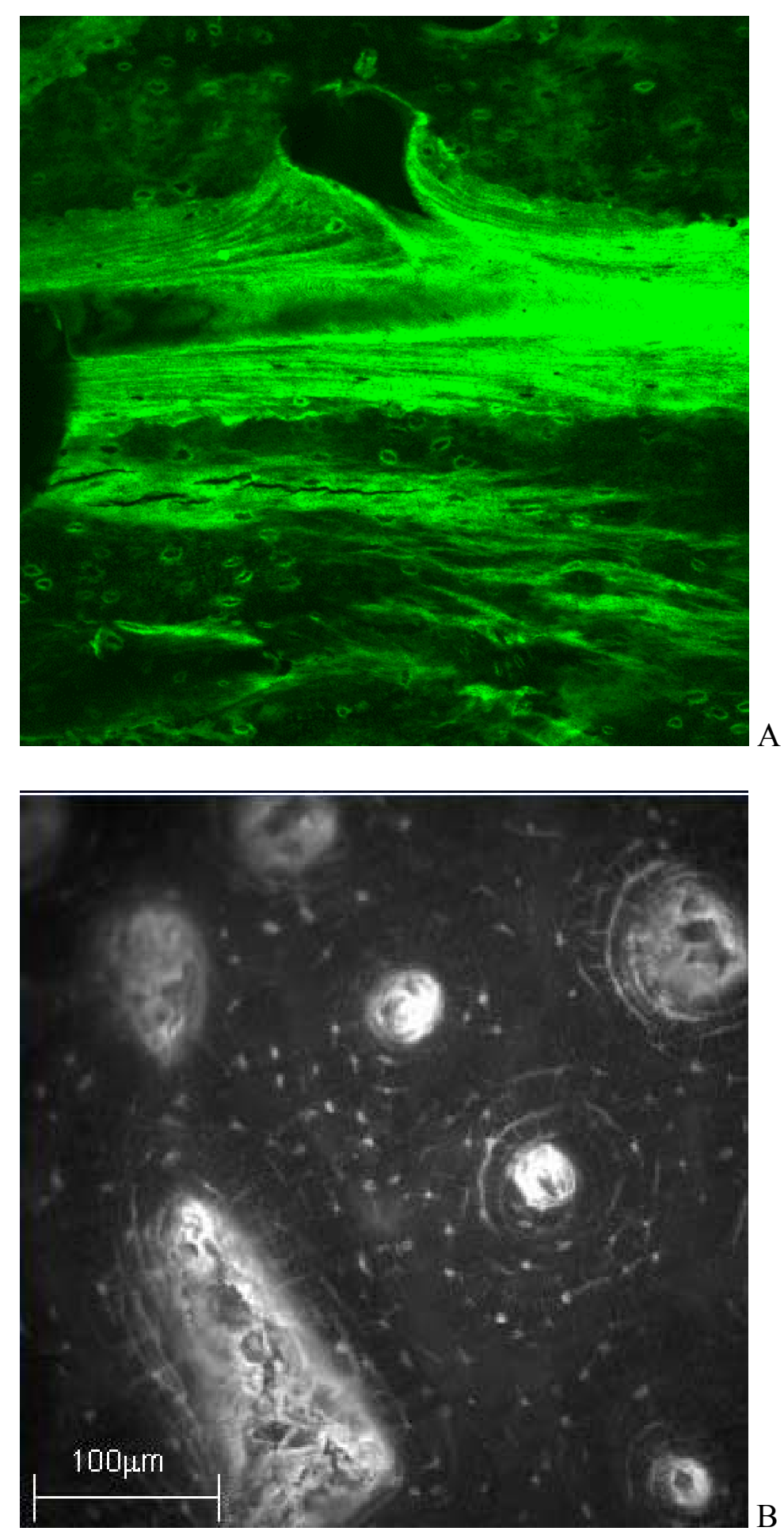

Figure 2.7. Typical micrograph demonstrating the longitudinal (A) and transverse (B) appearance of bone under LSCM. 


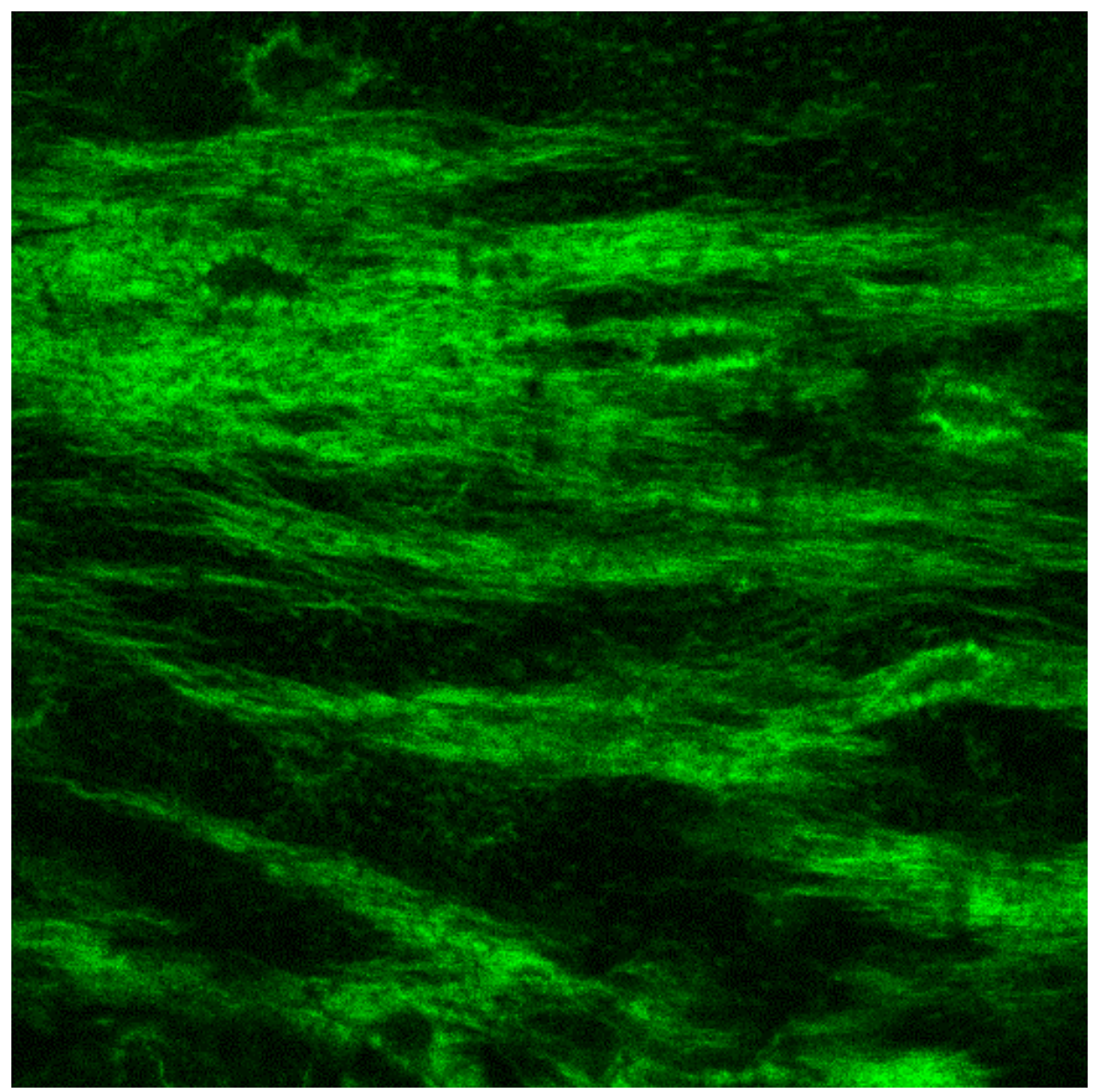

Figure 2.8. LSCM micrograph of the specimen surface oriented longitudinally to the direction of osteons with a tensile loading axis perpendicular to the horizontal direction. A typical appearance of diffusely damage areas demonstrating damaged regions in the canalicular network of lacunae (the scale bar is $30 \mu \mathrm{m}$ long). 

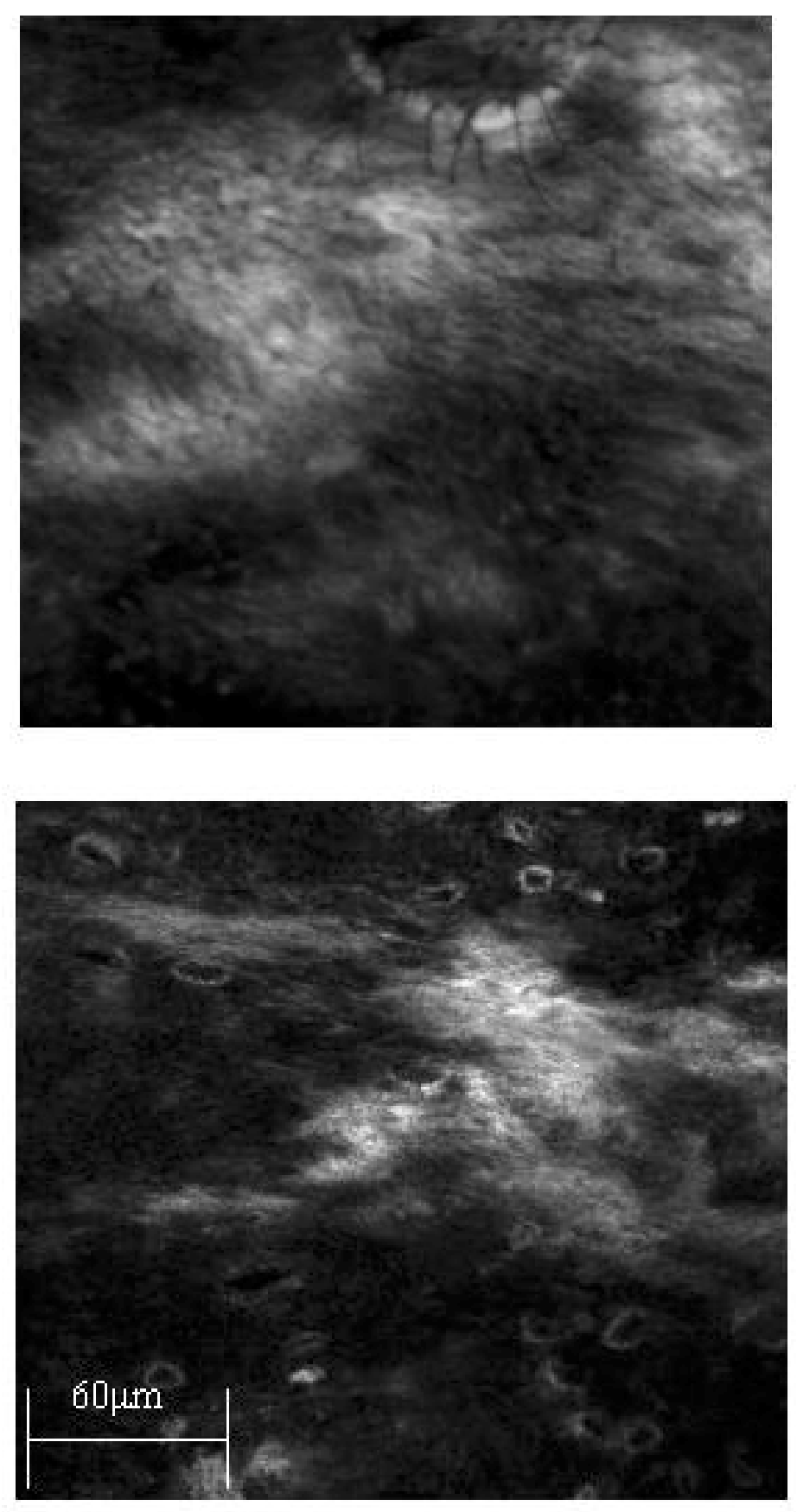

Figure 2.9. A confocal micrograph demonstrating pooled regions of stain in the intercanalicular spaces in-between neighboring lacunae. 


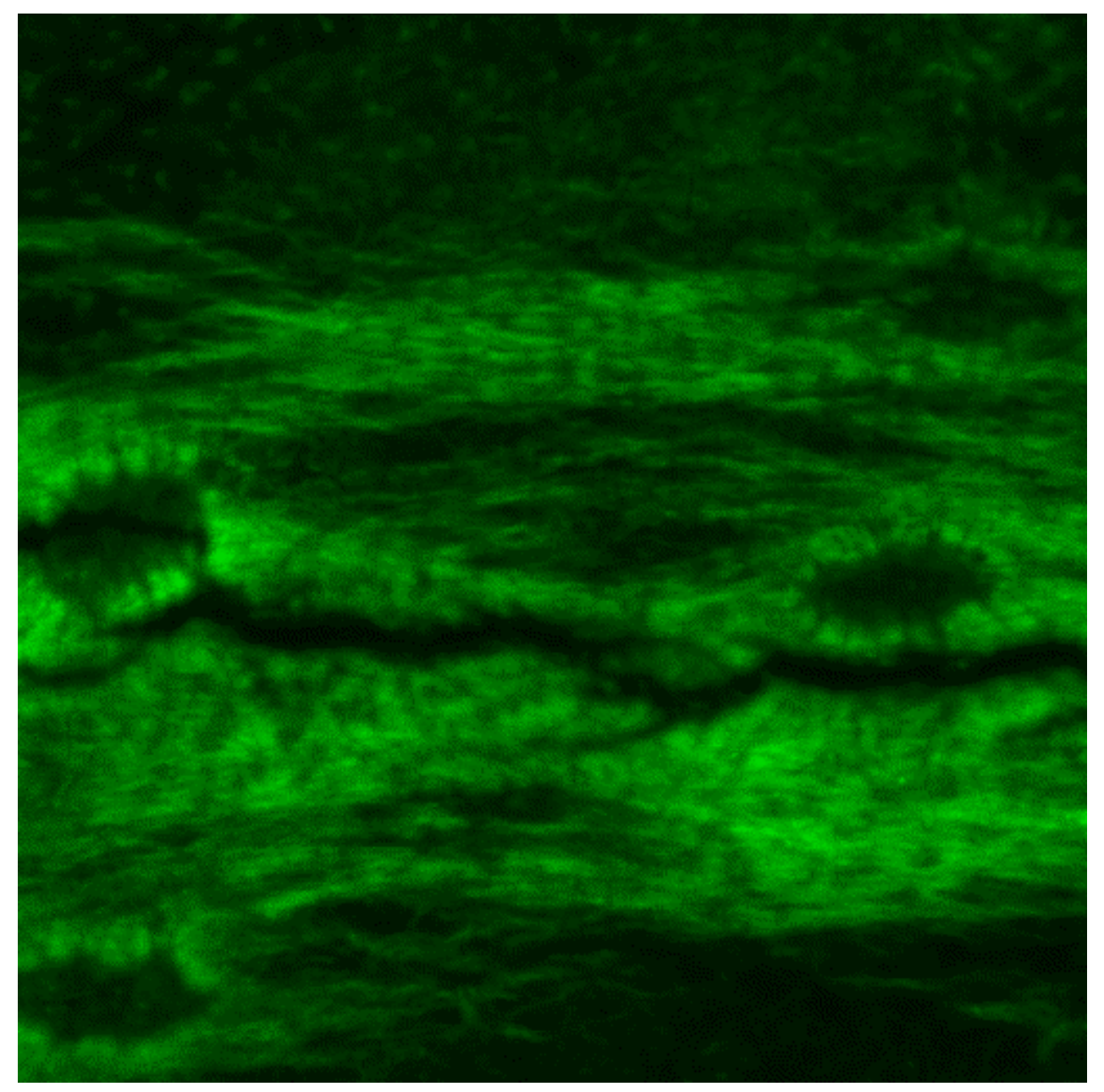

Figure 2.10. Confocal micrograph demonstrating the path of a crack with multiple deflections passing through a lacuna and passing around another one. This behavior is an indicator of the stress concentrating effects of lacunae (the scale bar is $20 \mu \mathrm{m}$ long). 


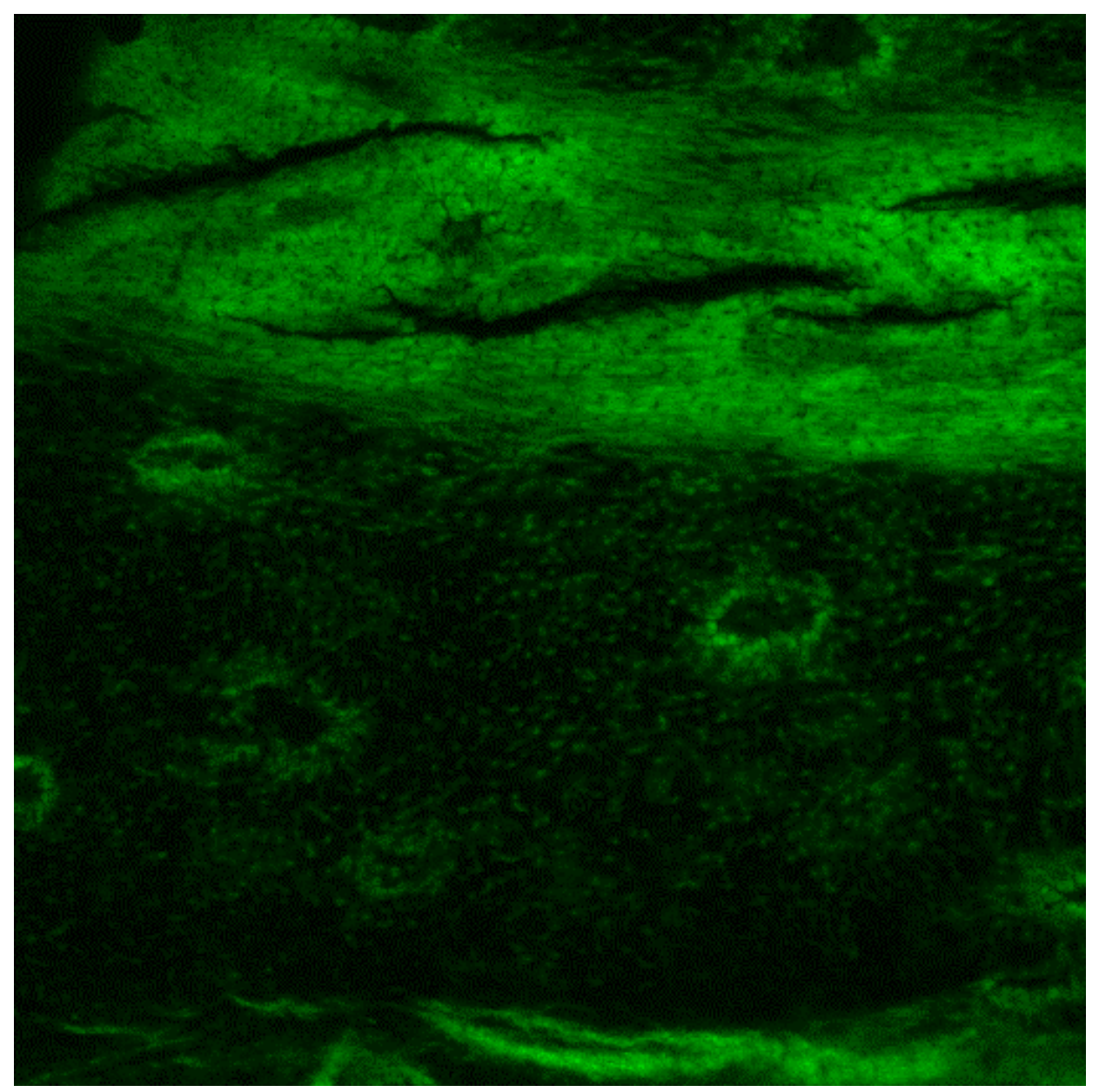

Figure 2.11. A confocal micrograph demonstrating the overlapping nature of microcracking, similar to other engineering materials that experience toughening behavior as a result of long discretized microcracks (the scale bar is $40 \mu \mathrm{m}$ long). 
Cyclic loading caused an increase in diffuse damage with a corresponding decrease of 5$10 \%$ in the initial stiffness $(198.23 \pm 27.35 \mathrm{~N} / \mathrm{mm})$. Critical loads, $\mathrm{P}_{\mathrm{C}}$, obtained from the load-deflection data (Figure 2.12) were used to calculate the corresponding stress intensity factors $\left(\mathrm{K}_{\mathrm{IC}}\right)$ that were plotted against Df.Dm.D (Figure 2.13). Fourth degree polynomial fitting was chosen for the nonlinear regression analysis of the data. Statistical analysis showed significant correlation between fracture toughness and diffuse damage $\left(\mathrm{r}^{2}=0.76, \mathrm{p}=0.0072\right)$.

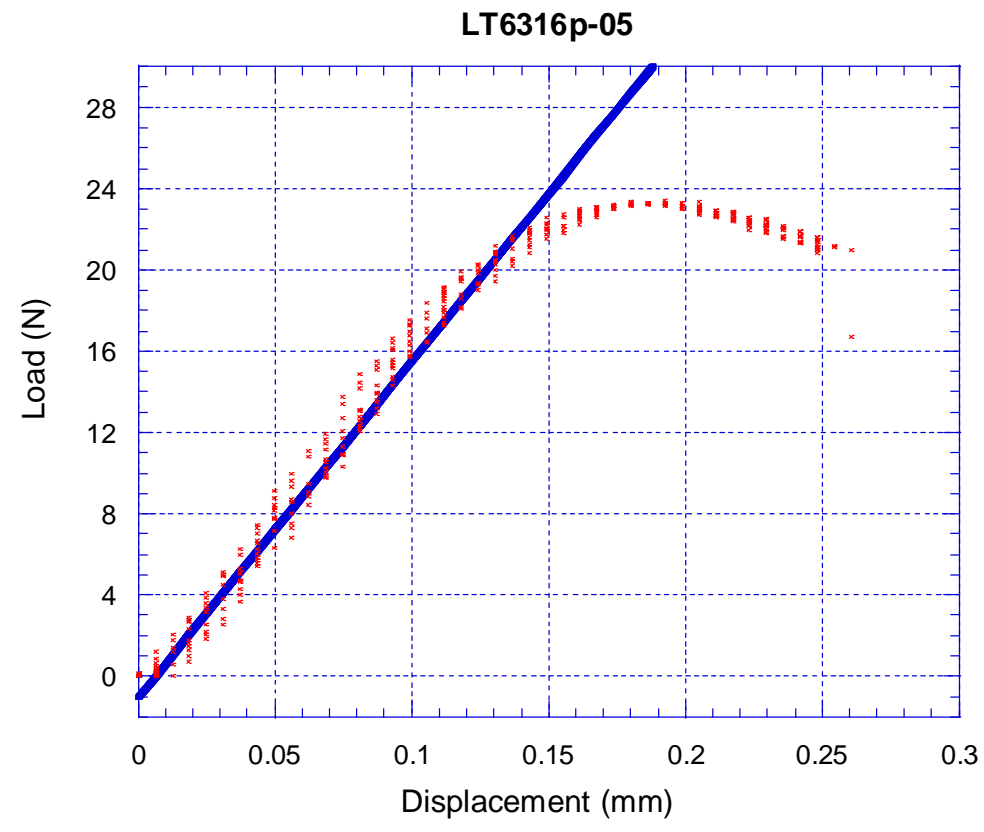

Figure 2.12. A graph representing a typical load vs. displacement relationship during monotonic loading of $\mathrm{CT}$ specimens. $\mathrm{P}_{\mathrm{C}}$ was found by superimposing a line parallel to the initial linear portion of the curve and having a $0.02 \%$ offset and determining the point of intersection with the load-displacement curve. 


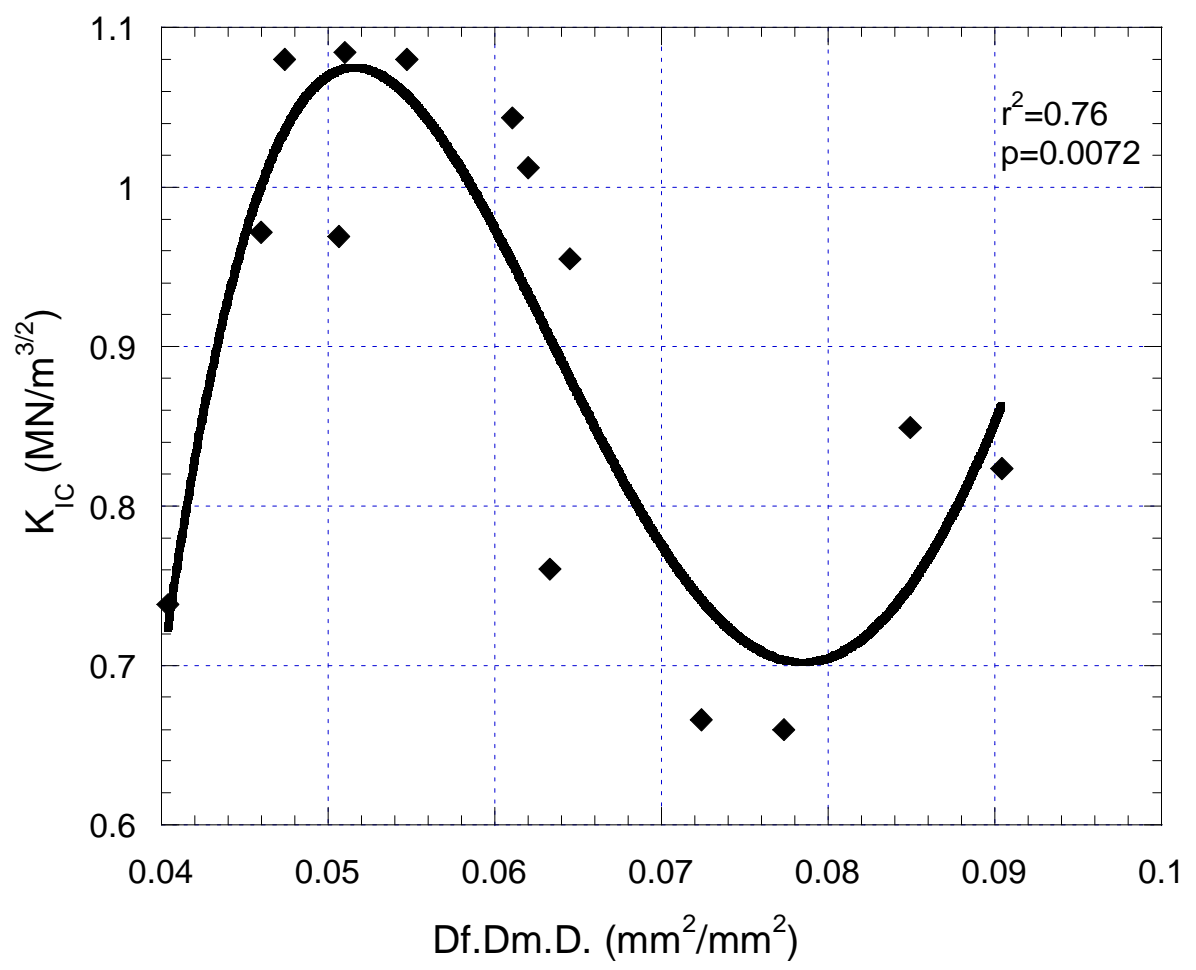

Figure 2.13. Graph representing the relationship between the fracture toughness $\left(\mathrm{K}_{\mathrm{IC}}\right)$ and diffuse damage (Df.Dm.D). The relationship is nonlinear and is given by:

$$
\mathbf{K}_{\mathbf{I C}}=-5.84 \times 10^{5} \mathbf{D}^{4}+1.89 \times 10^{5} \mathbf{D}^{3}-2.19 \times 10^{4} \mathbf{D}^{2}+1.08 \times 10^{3} \mathbf{D}-17.8
$$

Analysis of variance (ANOVA) demonstrated no statistically significant variation of morphological parameters $\left(\mathrm{OsD}=14.3643 \pm 1.9524, \# / \mathrm{mm}^{2} ; \% \mathrm{P}=7.3878 \pm 1.29\right.$, $\left.\mathrm{mm}^{2} / \mathrm{mm}^{2}\right)$ with either bone location (distal, medial, proximal) $\left(\mathrm{r}^{2}=0.07, \mathrm{p}=0.66 ; \mathrm{r}^{2}=0.31\right.$, $\mathrm{p}=0.13$ ) or cortex location (anteromedial, anterolateral, posterior) $\left(\mathrm{r}^{2}=0.041, \mathrm{p}=0.79\right.$; $\left.\mathrm{r}^{2}=0.04, \mathrm{p}=0.78\right)$ (Figure 2.14a,b). 

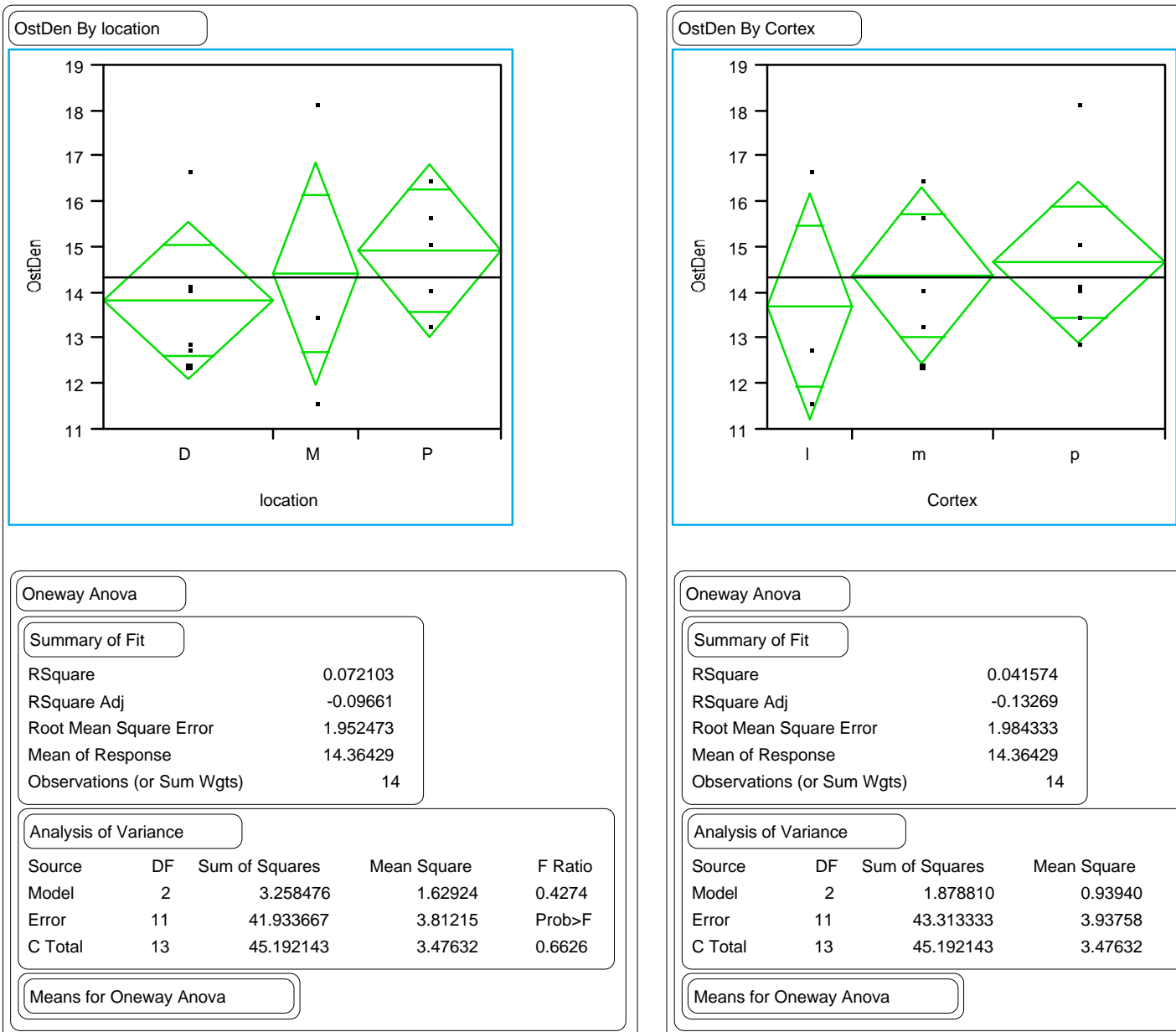

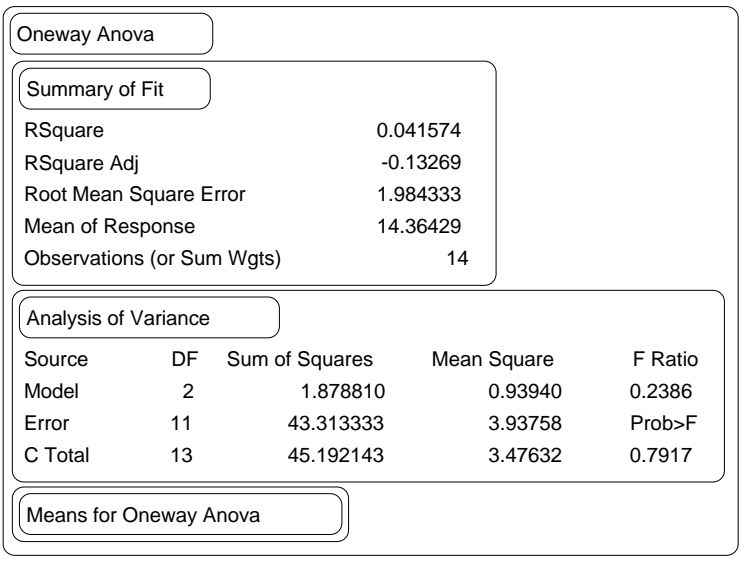

Figure 2.14a. Analysis of variance of Os.D. as a function of bone location and bone cortex location. 

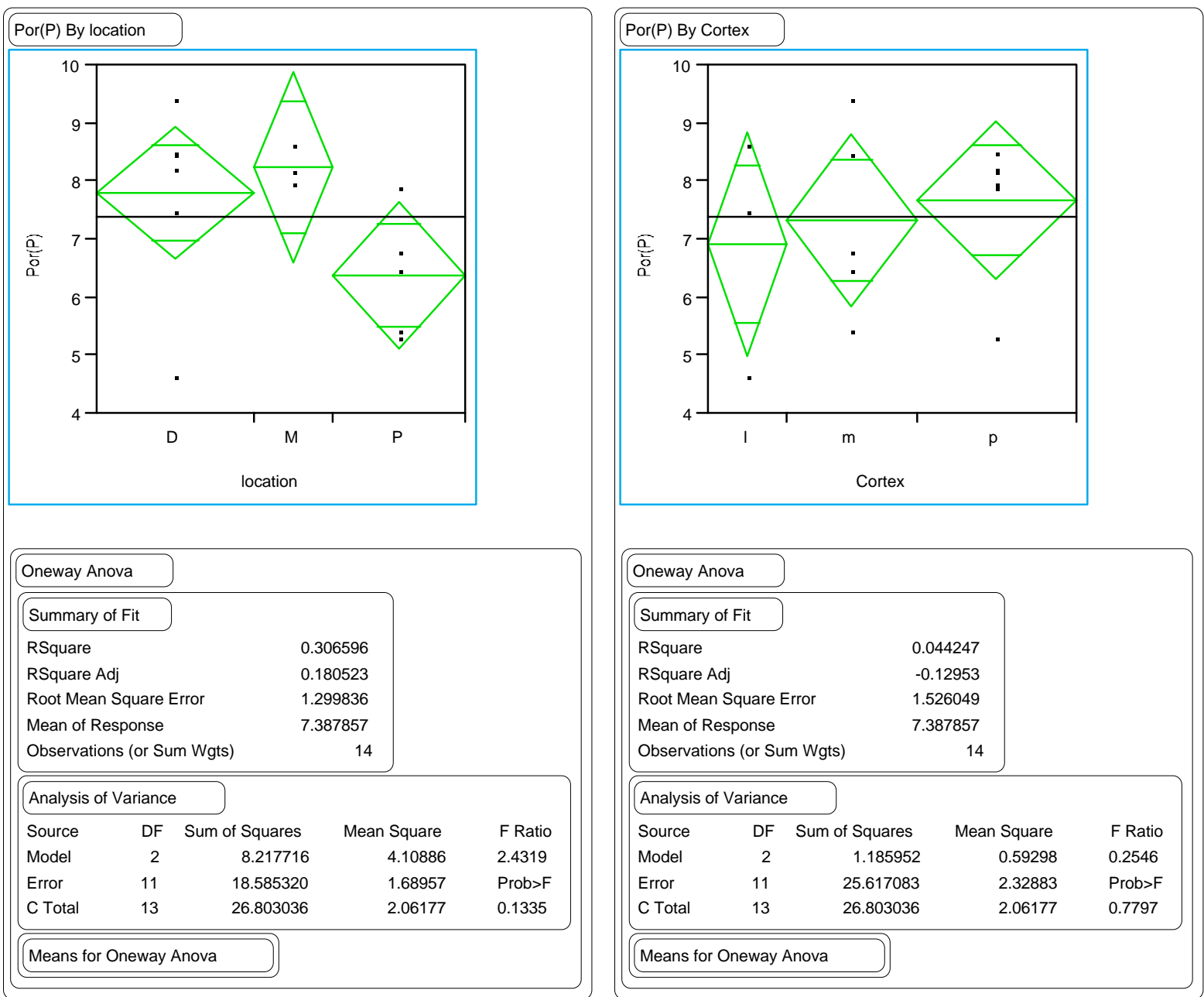

Figure 2.14b. Analysis of variance of $\mathbf{P}$ as a function of bone location and bone cortex location. 


\subsection{Discussion}

The characteristic time of all kinetic processes in solids, including phase transformation and breaking of bonds between the elements of microstructure, decreases very strongly with growing tensile stresses (Barenblatt and Botvina, 1983). Therefore, at the tips of the cracks where there is strong stress concentration, fracture processes are usually complicated by microstructural transformations, heat generation and microscopic damage accumulation. The damage process zone (DPZ) is referred to as the zone where interactions take place between microscopic flaws in the process of microcrack growth giving rise to strain localization within this narrow band. By studying the DPZ of a material it is possible to identify the accurate failure behavior of a material and by characterization of the compositional configurations of the microstructure, such as voids, inclusions, grain boundaries, etc. it is possible to identify microarchitectural sites that are responsible for crack initiation and/or serve as an effective restraints on crack propagation resulting in enhanced material toughness.

Our observations suggest that diffuse damage in cortical bone is a result of breakdowns in the canalicular network (Figure 2.9). The fractures of hydroxyapatite crystals surrounding collagen fibers or delaminations at the crystal-fiber interfaces could provide a possible explanation of the onset of these damaging processes. Structural delaminations as a result of the damaging events generate micro-vacuum regions, which subsequently cause a stain uptake. Those delaminations are very small; therefore, those newborn regions appear blurry and pooled (Figure 2.9). Growth of those defects tends to open up lacunae in a direction perpendicular to the local stresses. It was interesting to observe that spatial distribution of lacunae and the canalicular network interconnecting those lacunae often caused cracks to deflect from their paths upon meeting with lacunae (Figure 2.10) thus leading to the formation of discretized microcracks (Figure 2.11). Overall histological observations could be summarized into the following possible micromechanism of damage evolution: damage onset, as a result of fractures in crystals within the intra-canalicular matrix; subsequent growth into intra-canalicular cracks, as a result of possible delaminations between collagen bundles; and multiple deflections of those cracks, due to three-dimensional distribution of canaliculi and lacunae and the stress concentrating effects of lacunae. 
Results from fracture toughness tests demonstrated that sub-microscopic damaging events are the mechanisms behind the stiffness degradations in early stages of fatigue proposed in the studies by Burr et al. (1998) and Schaffler et al. (1996). Results indicated that bone exhibits a toughening behavior as a function of diffuse damage (Figure 2.13). The explanation for this behavior lies within the frontal damage zone formed around the crack tip and the energy consumption processes within this zone (Becher, 1991; Budianski et al., 1983; Faber and Evans, 1983a,b; Mai, 1998). Strain energy associated with the formation of flaws, as a result of damaging events, serves as the toughening increment. The first rising phase in Figure 2.13 indicates that, as diffuse damage accumulates, bone toughens until it reaches some "threshold" value of damage when microcracks first start to appear resulting in a decrease of toughness (falling phase of the graph in Figure 2.13). Then, more diffuse damaging processes and the interactions of those microflaws cause an increase in toughness again (second rising phase in Figure 2.13). This phenomenon could be a part of the behavior that toughness experiences as a function of the number of microcracks. But since the goal of this study was to investigate bone's response to diffuse damage accumulation and the damaging loading was stopped prior to the appearance of major cracks, the relationship between toughness and the number of cracks was not investigated.

In general there are two major classes of restraints identified to describe the toughening interactions in materials. The first involving purely geometrical processes, deflections along or across weak interfaces, etc. The impeding influence of the responsible microstructural elements regarded as "transitory obstacles' lasts only for the duration of crack front intersection hence these sources of toughening are relatively ineffective. In other words, 'intersections' with otherwise inactive microstructural heterogeneities in the elastic field can cause a planar crack to deflect from its path and absorb additional energy, effectively increasing the net resistance or toughness. But the crack retains no memory of any such intersection once the intersection is complete. The second class of restraint comprises shielding processes. The critical interactions occur away from the tip, within the DPZ. The most consequential are those with a large component of irreversibility, so that the restraining influence persists, via a pervasive 
state of residual closure strain, in the crack "wake". Because of their cumulative nature, shielding processes are potentially stronger sources of toughening.

For brittle materials, unstable crack propagation occurs when the crack driving force, the strain energy release rate reaches a critical value. For several other classes of materials, the existence of DPZ results in stable crack growth prior to peak load. Such behavior is often referred as R-curve behavior. The R-curve characterizes the resistance to fracture of a given material during incremental slow-stable crack growth. Vashishth and co-workers (1997) utilized this approach and demonstrated toughening behavior associated with macrocrack extension and with the increase in the number of microcracks both for bovine and human cortical bone. But the nature of the cumulative processes of damage and its relationship with microstructural configurations of the mineralized tissue remained unanswered.

The toughening behavior at the ultra-structural level, demonstrated in this study raises interesting possibilities of biological responses in bone. This behavior could provide an adequate "time" for a biological response prior to the growth of those submicroscopic flaws and the subsequent catastrophic irreversible effects in the material's behavior. It was also interesting to observe that the threshold values of DfDmD were $\approx 5 \%$, which was very close to the amount of in-vivo, diffuse damage $(\approx 3 \%)$ (Norman et al., 2000) supporting the idea that damage serves as a constant maintenance stimulus of bone (Martin and Burr, 1989; Frost, 1973; Currey, 1984; Carter, et al., 1987).

It was mentioned earlier that octeocytes that are trapped within lacunae serve as the mechanical loading sensors. The presence of cracks in a fluid-saturated bone could have a significant effect on the overall elastic moduli depending on the degree of interconnection between cracks (O’Connel and Budiansky, 1977). When fluid fills crack like voids in the fluid-saturated solid, this process results in dissipation of energy by either a viscous relaxation mechanism (due to the relaxation of shear stresses in the viscous fluid) or by fluid flow (resulting from the stress-induced fluid flow between cracks). Depending on the crack density and the frequency of the applied stress field effective viscoelastic moduli are modified. Therefore the proposed micromechanism in this study of sub-microscopic damage evolution together with observed variations of 
fracture toughness as a function of damage at the ultrastructural level serves as a possible explanation of the mechanisms behind the stiffness loss.

Finally, overall observations have led us to propose our hypothetical mechanism of the intracortical remodeling. Osteons operate as networks of "spider webs" with the bone lining cells serving as "spiders". Damage behavior in interlacunar spaces would lead to strain localizations, which would be sensed by alterations in strain patterns in the neighboring unaffected osteocytes, which are still communicating with bone lining cells. These alterations in the deformation behavior of healthy osteocytes would be sensed by bone lining cells and an appropriate remodeling response could be triggered. Spiders sense the presence of a prey in their webs by the alterations of the straining signals from the numerous wires. And interestingly, they ignore the signals caused by wind-generated deformation of the web. We believe that the "mechanism" by which they distinguish the presence of the prey from that of caused by wind is similar to that of the communication in-between osteocytes and bone lining cells. In addition, relatively small size of the damage patterns in the interlacunar spaces could be the reason why it has not been identified in animal studies of damage caused remodeling. 


\section{CHAPTER 3}

\section{DAMAGE EVOLUTION IN 3-POINT BENDING OF HUMAN CORTICAL BONE}

(Parsamian, G.P. et al., 2001. $47^{\text {th }}$ Ann. Meet. ORS.)

\subsection{Introduction}

Continuum mechanics and thermodynamics constitute the basic theoretical tools for the formulation of the physical phenomena of deformation and fracture. For numerous practical reasons researchers approach the problem of modeling each broad class of phenomena separately. The phenomenological method of the identification and rheological classification of solids is based on experimental results and provides only a frame of reference for general characteristics. It consists of studying the volume element of matter through the relations between cause and effect, which exist between the physically accessible variables constituting the input and output of the process under study. This approach is sufficient for the qualitative characterization of materials but it does not allow, except for the linear phenomenon, constitution of the constitutive laws of materials.

The rheological identification of materials usually involves uniaxial loading of specimens of standardized geometry and identification of nonlinear material response using stress-strain behavior. Typical nonlinear responses of materials are plastic, viscoplastic, viscoelastic responses (Figure 3.1).

Materials with completely different nonlinear material response mechanisms can show identical stress-strain behavior. This was mentioned by Robotnov (1980), who argued that curves obtained from loading behavior of two materials: one deforming according to classical flow plasticity and one, what he referred as "pseudoplasticity" show identical behavior. He mentioned that the difference between those behaviors becomes evident only when the material is unloaded. The classical plastic material unloads along a straight line with the same slope as its elastic modulus, while the "pseudoplastic" material unloads along a straight line back to the origin (Figure 3.2). 

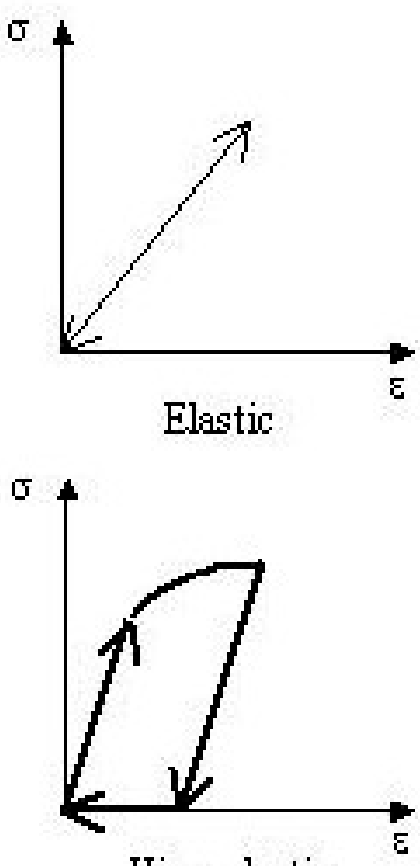

Viscoelastic

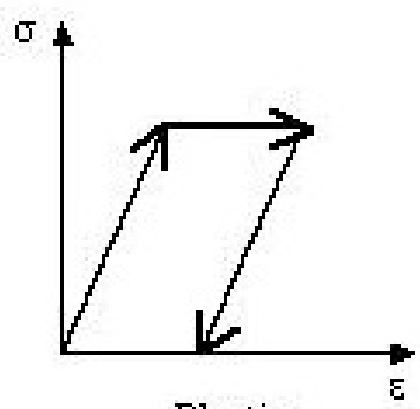

Plastic

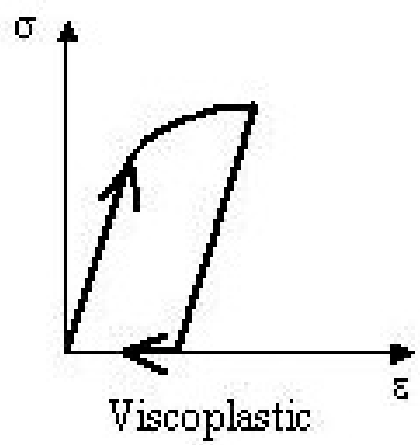

Figure 3.1. Typical stress-strain behavior of elastic, plastic, visoelastic and viscoplastic materials. In elastic materials unloading follows the same loading path. In plastic materials material unloads with the same modulus leading to unrecoverable plastic residual strain. In viscoelastic materials residual strains completely recover upon removal of load. In viscoplastic materials residual strains do not remain recover completely upon removal of load.

The differences in behavior of those materials are caused by two distinct physical mechanisms, with former deforming by a slip mechanism and dislocation motion and later deforming as a result of developments of cracks in an otherwise purely elastic material. In the case of plastic materials microstructural alterations caused by slipping mechanisms are unrecoverable and the resultant plastic strains are unrecoverable upon unloading. For the "pseudoplastic" materials, cracks close during unloading leading to the complete recovery. In addition, classical plastic materials are rate independent (Lemaitre and Chaboche, 1990), therefore, during reloading after unloading the 
compliance of the material will not change, whereas "pseudoplastic" materials will demonstrate an increase in compliance as a result of material's "softening" caused by cracks.

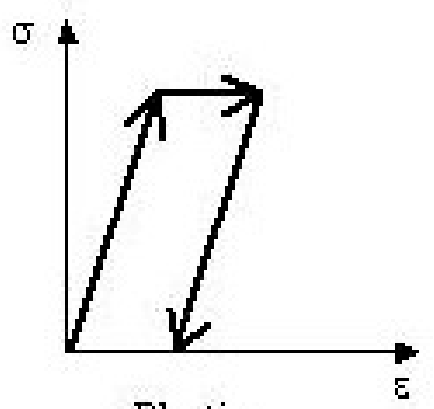

Plastic

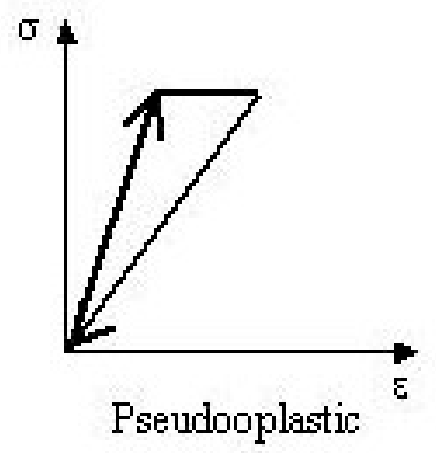

Figure 3.2. Stress strain relationships for plastic and pseudoplastic materials. Plastic behavior demonstrates characteristic residual strains after unloading, whereas pseudoplastic material unloads to its original configuration.

Sedlin (1965) was the first to publish a study involving unloading of cortical bone specimens. He investigated the loading and unloading behavior of cantilever beam specimens of human cortical bone. The results indicated slight hysteresis effects and small residual strains upon unloading. Bonfield and Li (1966) conducted tensile loading and unloading of bovine cortical bone specimens under strain control with progressively increasing loads until rupture. They have concluded that residual strains after unloading were plastic strains. Burstein et al. (1973) performed similar experiments with an exception of higher strain rates and concluded that bone behaves like a truly plastic material. Other studies of similar nature include studies by Lewis and Goldsmith (1975), Knets et al. (1976), Melnis and Knets (1981) and others. Overall, all researchers have demonstrated the development of nonlinear strains during loading and their recovery behavior after unloading.

Fondrk and co-workers (1988) published a study where they have addressed the issue of the post-yield characterization of cortical bone in more detail. They demonstrated 
that bone responds to low magnitude stresses primarily as a linear viscoelastic material with the recovery of residual strains after the unloading. The deformation response to higher stresses was creep dominated. They identified the threshold value of the stress that separated those two behaviors. This study had lead them to conclude that the observed reduction of stiffness in the subsequent reloading ramps together with the recovery of the majority $(\approx 70 \%)$ of the residual strains was a consequence of damaging mechanism behind the creep deformation. Recently, Jepsen and co-workers (1997) utilized similar loading protocol and investigated the damaging processes in bone under shear loading. They defined the ratio of unloading shear modulus and loading shear modulus as a damage parameter and performed series of torsional relaxation cycles. Failure properties, determined from pre- and post-damage relaxation cycles demonstrated that yield stress and strain, the relaxation rate and total relaxation exhibited 5-10 fold greater degradation than both strength and modulus when tested at higher strain rates. In the subsequent study they investigated microcracking mechanisms involved in the damaging processes and concluded that damage density, measure as the number of cracks per square millimeter was correlated with stiffness degradation and changes in relaxation (Jepsen et al., 1999). Also, based on microscopic observations of damage patterns, they concluded that lamellar interface plays an important role in delaying microcrack coalescence and delays the inevitable formation of the fatal cracks.

The cumulative nature of damage under fatigue loading conditions has received much more attention among bone researchers. Carter and Hayes (1977) investigated the uniaxial tensile fatigue behavior of wet bovine cortical bone and demonstrated that specimen stiffness undergoes a gradual loss process under low-cycle fatigue conditions. They identified the similarities of this behavior with the behavior of engineering materials (e.g. microcracking, debonding, void growth and fiber rupture) and also demonstrated the progressive increase in the hysteresis as a result of the increasing nonlinear behavior. Based on their experimental observations they proposed a cumulative damage model, which enabled them to describe the combined time-dependent and cycledependent fracture of devitalized compact bone (Carter and Caler, 1985; Caler and Carter, 1989). The major assumption was that the failure was interpreted based on linearlife fraction rule, calculated using Miner-Palmgren theory. But recent studies of Zioupos 
and co-workers have demonstrated that damage accumulation does not follow Miner's linear theory of accumulation, instead it is a nonlinear function of cycle number (Zioupos et al., 1996a,b; 1998) similar to that of suggested by Chaboche for some classes of engineering materials (Lemaitre and Chaboche, 1990).

\subsection{Objectives}

The incomplete understanding of damaging processes is greatly responsible for the absence of unique constitutive relations for bone, the importance of which is unarguable for the application of engineering methods including prosthesis design, description of adaptive changes and studying fracture causes and behavior. The main goal of this portion of the study is to utilize the loading-unloading-reloading testing procedure to investigate damage evolution in three-point bending of human cortical bone beam specimens. The loading of beams with a concentrated load generates a complex loading modes throughout the depth of the specimens. The two specific issues to be investigated are: 1) characterization of the influence of the interactions of three major modes of loading on the failure behavior and 2) characterization of damage evolution. Specifically, by varying the aspect ratios of the beams to be tested study the influence of shearing stresses on failure behavior. Also, investigate the evolution properties of various damage parameters. The damage parameters would be defined as: i) the ratio of damaged stiffness to the virgin stiffness and ii) the ratio of the residual strain to the total strain. 


\subsection{Materials and Methods}

\subsubsection{Specimen Preparation}

Four sections, approximately $5 \mathrm{~cm}$ long were cut from each of the pair of tibia obtained from 58-year-old male donor, who had died from causes unrelated to skeletal conditions. Sections were cleaned of marrow, wrapped with $0.9 \%$ saline soaked gaze and kept in airtight plastic containers at $-20^{\circ} \mathrm{C}$ until machining. Forty-two rectangular beam specimens were wet machined in pairs from anteromedial, anterolateral and posterior regions of the tibia. Table 3.1 summarizes the specimen geometries and the locations from which they were obtained. Fourteen specimens $(n=14)$ from anterolateral (4 pairs) and posterior ( 3 pairs) cortices of the right tibia were separated into two groups (G1 and G2) by randomly selecting one from each pair of specimens.

\subsubsection{Mechanical Testing}

The major objective in this portion of the study was to investigate the interactions of tensile, compressive and shear forces on the failure behavior of beams. To accomplish this beams were tested in 3-point bending at two different aspect ratios (the ratio of support span to beam's height). G1 was tested at approximately 20:1 and G2 was tested at 10:1. The loading protocol consisted of loading, unloading and reloading at a preset strain rate under displacement control. The fundamental idea was to investigate the post-yield behavior and failure characteristics and to examine the damage patterns at the midspan of beams, close to the failure surface and in the overhanging portions of beams. 


\begin{tabular}{|c|c|c|c|c|c|c|c|}
\hline \multicolumn{5}{|c|}{ Cadaver \# 996129} & \multicolumn{2}{|c|}{58 Male } & \\
\hline \multicolumn{8}{|c|}{ Right Tibia } \\
\hline Specimen & Location & Cortex & Length(L) & Height (D) & Width (B) & Mom Inert. (2x4) & Mom Inert (4x2) \\
\hline RDL01 & Distal & L & 48 & 2.44 & 4 & 4.842261333 & 13.01333333 \\
\hline RDL02 & Distal & $\mathrm{L}$ & 48 & 2.38 & 4 & 4.493757333 & 12.69333333 \\
\hline RDP03 & Distal & $\mathrm{P}$ & 48 & 2.12 & 4 & 3.176042667 & 11.30666667 \\
\hline RDP04 & Distal & $\mathrm{P}$ & 48 & 1.95 & 4 & 2.471625 & 10.4 \\
\hline RDM05 & Distal & $M$ & 48 & 2.13 & 4 & 3.221199 & 11.36 \\
\hline RDM06 & Distal & $M$ & 48 & 2.04 & 4 & 2.829888 & 10.88 \\
\hline RMDL07 & Med/Distal & $\mathrm{L}$ & 48 & 2.12 & 4 & 3.176042667 & 11.30666667 \\
\hline RMDL08 & Med/Distal & $\mathrm{L}$ & 48 & 2.18 & 4 & 3.453410667 & 11.62666667 \\
\hline RMDM09 & Med/Distal & $M$ & 48 & 2.1 & 4 & 3.087 & 11.2 \\
\hline RMDM10 & Med/Distal & $\mathrm{M}$ & 48 & 2.09 & 4 & 3.043109667 & 11.14666667 \\
\hline RMDP11 & Med/Distal & $\mathrm{P}$ & 48 & 2.15 & 4 & 3.312791667 & 11.46666667 \\
\hline RMDP12 & Med/Distal & $\mathrm{P}$ & 48 & 2.15 & 4 & 3.312791667 & 11.46666667 \\
\hline RMPL13 & Med/Proximal & $\mathrm{L}$ & 48 & 2.07 & 4 & 2.956581 & 11.04 \\
\hline RMPL14 & Med/Proximal & $\mathrm{L}$ & 48 & 2.15 & 4 & 3.312791667 & 11.46666667 \\
\hline RMPM15 & Med/Proximal & $M$ & 48 & 2.16 & 4 & 3.359232 & 11.52 \\
\hline RMPM16 & Med/Proximal & $\mathrm{M}$ & 48 & 2.08 & 4 & 2.999637333 & 11.09333333 \\
\hline RMPP17 & Med/Proximal & $P$ & 48 & 2.12 & 4 & 3.176042667 & 11.30666667 \\
\hline RMPP18 & Med/Proximal & $\mathrm{P}$ & 48 & 2.12 & 4 & 3.176042667 & 11.30666667 \\
\hline RPL19 & Proximal & $\mathrm{L}$ & 48 & 2.2 & 4 & 3.549333333 & 11.73333333 \\
\hline RPL20 & Proximal & $L$ & 48 & 2.22 & 4 & 3.647016 & 11.84 \\
\hline RPM21 & Proximal & $M$ & 48 & 2.07 & 4 & 2.956581 & 11.04 \\
\hline RPM22 & Proximal & $\mathrm{M}$ & 48 & 2.1 & 4 & 3.087 & 11.2 \\
\hline RPP23 & Proximal & $\mathrm{P}$ & 48 & 2.18 & 4 & 3.453410667 & 11.62666667 \\
\hline \multicolumn{8}{|c|}{ Left Tibia } \\
\hline LDP01 & Distal & $P$ & 48 & & & & \\
\hline LDM02 & Distal & $\mathrm{M}$ & 48 & 2.17 & 4 & 3.406104333 & 11.57333333 \\
\hline LDM03 & Distal & $M$ & 48 & 2.22 & 4 & 3.647016 & 11.84 \\
\hline LMDM04 & Med/Distal & $\mathrm{M}$ & 48 & 2.18 & 4 & 3.453410667 & 11.62666667 \\
\hline LMDM05 & Med/Distal & $\mathrm{M}$ & 48 & 2.13 & 4 & 3.221199 & 11.36 \\
\hline LMDL06 & Med/Distal & $\mathrm{L}$ & 48 & 2.11 & 4 & 3.131310333 & 11.25333333 \\
\hline LMDL07 & Med/Distal & $L$ & 48 & 2.16 & 4 & 3.359232 & 11.52 \\
\hline LMDP08 & Med/Distal & $\mathrm{P}$ & 48 & 2.16 & 4 & 3.359232 & 11.52 \\
\hline LMPL09 & Med/Proximal & $\mathrm{L}$ & 48 & 2.15 & 4 & 3.312791667 & 11.46666667 \\
\hline LMPL10 & Med/Proximal & $\mathrm{L}$ & 48 & 2.11 & 4 & 3.131310333 & 11.25333333 \\
\hline LMPM11 & Med/Proximal & $M$ & 48 & 2.15 & 4 & 3.312791667 & 11.46666667 \\
\hline LMPM12 & Med/Proximal & $M$ & 48 & 2.13 & 4 & 3.221199 & 11.36 \\
\hline LMPP13 & Med/Proximal & $P$ & 48 & 2.15 & 4 & 3.312791667 & 11.46666667 \\
\hline LMPP14 & Med/Proximal & $P$ & 48 & 2.15 & 4 & 3.312791667 & 11.46666667 \\
\hline LPM15 & Proximal & $\mathrm{M}$ & 48 & 2.1 & 4 & 3.087 & 11.2 \\
\hline LPM16 & Proximal & $M$ & 48 & 2.19 & 4 & 3.501153 & 11.68 \\
\hline LPL17 & Proximal & $\mathrm{L}$ & 48 & 2.11 & 4 & 3.131310333 & 11.25333333 \\
\hline LPL18 & Proximal & L & 48 & 2.17 & 4 & 3.406104333 & 11.57333333 \\
\hline LPP19 & Proximal & $\mathrm{P}$ & 48 & 2.13 & 4 & 3.221199 & 11.36 \\
\hline
\end{tabular}

Table 3.1. Summary of specimens to be used in this portion of the study. 
In order to measure strains at the outermost (tensile) fibers of beams, two retroflective markers were attached approximately $4 \mathrm{~mm}$ apart centered at midspan axis of the surface perpendicular to loading direction. Specimens were tested in a custom made 3-point bending fixture (length of the support span=40mm) on MTS sevohydraulic testing machine (Figure 3.3). A constant displacement rate of $166.7 \mu \mathrm{m} / \mathrm{sec}$ was applied for both loading and unloading phases resulting in strain rate of $0.00375 \mathrm{~s}^{-1}$ (Eq. 3.2). Loading and unloading was prescribed using a special function generator. Loading amplitudes were progressively increased until the specimens would fail. Loaddeformation data was collected on the personal computer using LabTek Notebook data acquisition software. Strain measurements were made using a laser extensometer (Electronic Instrument Research, Irwin, PA).

After testing, failed specimens were stained with basic fucsin in the desscending concentrations of alcohol $(100 \%, 90 \%, 80 \%$ and $70 \%)$. After staining a $250 \mu \mathrm{m}$ thick sections were removed from the cross section of beams. One section was removed from immediately adjacent to the failure surface and one from the overhanging portion of the beam specimens resulting in two sections for each specimen. Those sections were subsequently polished to a mirror finish with progressively finer grades of sand paper and mounted on glass slides for microscopic observations. A LSCM system was used to study damage patterns in those sections. 


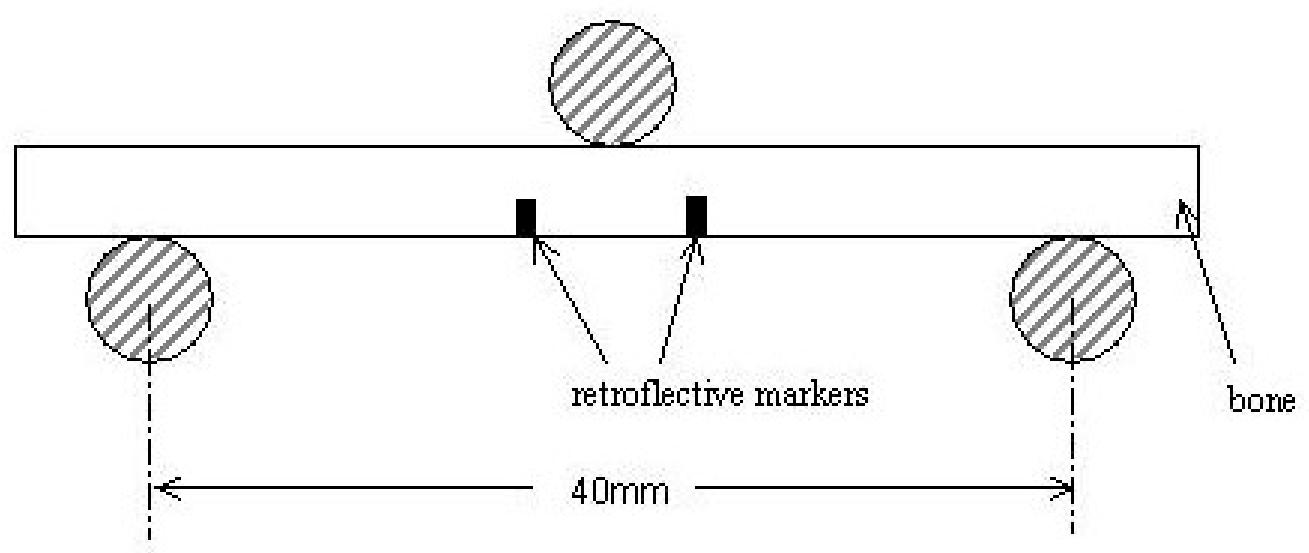

Figure 3.3. Schematic diagram of 3-point bending fixture indicating the locations of retroflective markers.

\section{Remark 1.}

Bernoulli's Beam Theory was used to calculate stresses and strains at the outermost fibers of beams:

$$
\sigma=\frac{P L c}{4 I}
$$

and

$$
\varepsilon=\frac{12 c d}{L^{2}}
$$

where $P$ is the load, $L$ is the span length, $d$ is the deformation, $I$ is the second moment of inertia around the bending axis and $c$ is the distance from the cross-sectional center of mass.

Remark 2.

Strains from the relative deformations of the retroflective markers were calculated using:

$$
\varepsilon=\left(I-I_{0}\right) / I_{0}
$$


where $I_{0}$ and $I$ represent the distance between the markers initially and after during loading, respectively.

\subsection{Results}

\subsubsection{Failure behavior}

Confocal Microscopy gives a definite advantage in studying damage behavior due to its' high signal to noise ratio. In the previous section, we have demonstrated that damage initiates at the lacunae canalicular level. Observations made from bone sections that were obtained from a transverse direction of cortical bone supported our conclusions of observations made from the longitudinal sections of bone in the previous chapter. Figure 3.4 demonstrates a confocal micrograph of an undamaged osteon and a micrograph of an osteon with extensive damage in the lacunae canalicular network. As a result of the appearance of microcracks, those newborn "cavities" are filled with "larger" amounts of stain and therefore appear brighter under laser excitation.
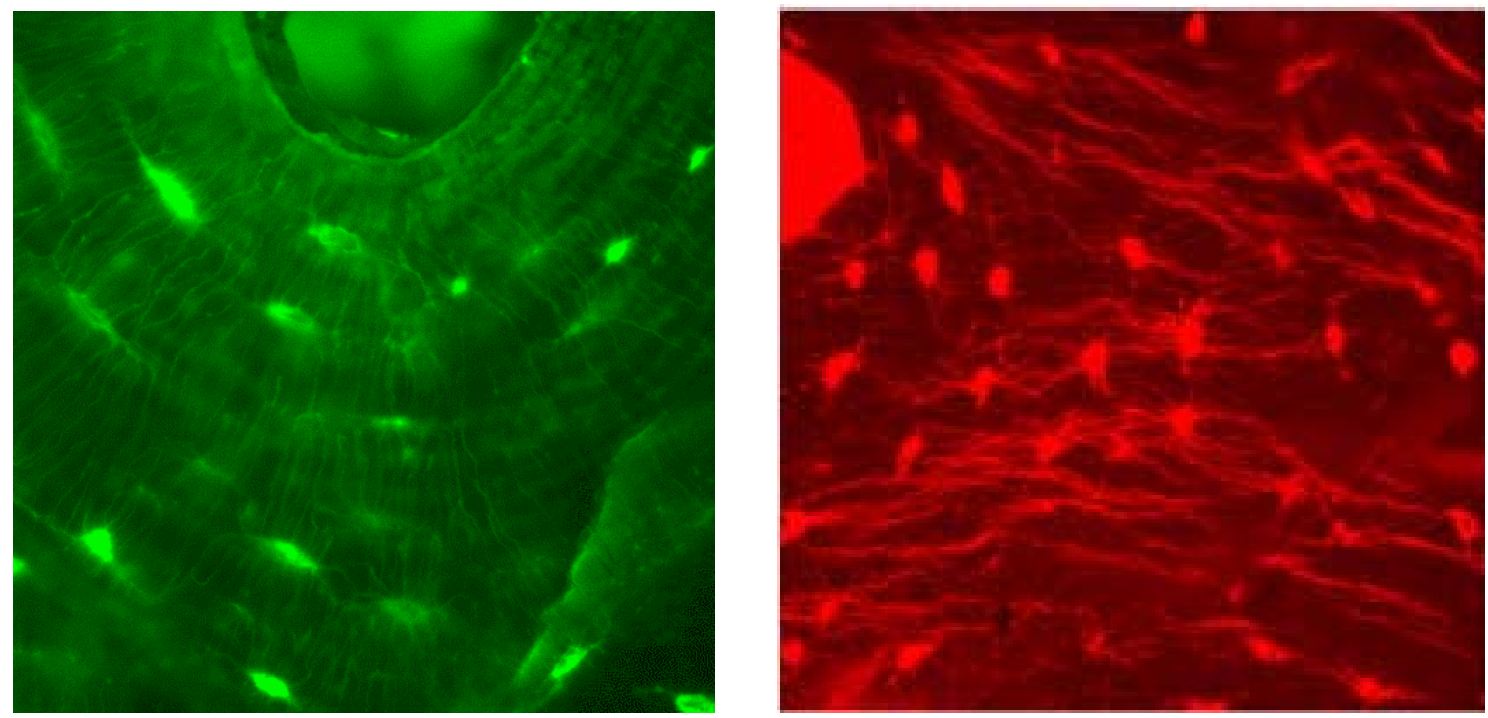

Figure 3.4. Confocal micrographs demonstrating nondamaged lacuna-canalicular network(left) and damaged canalicular network.

Figure 3.5. demonstrates characteristic damage patterns observed in tensile and compressive regions of the specimens. Tensile regions were generally more damaged than the compressive areas. Thus supporting the idea that bone is considerably weaker in 
tension than in compression. The dominant type of microcracking patterns correspond to interlamellar and cement line debonding and matrix cracking.
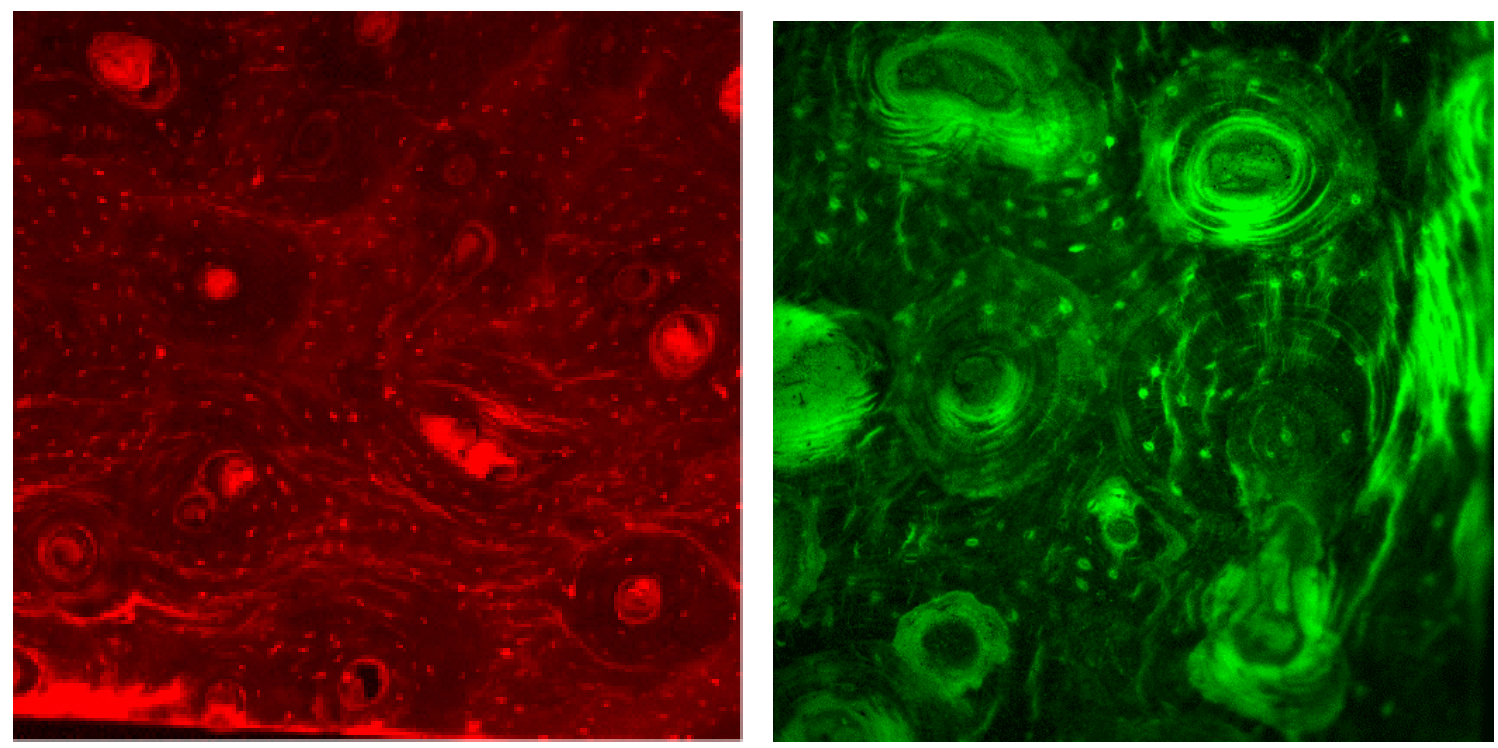

Figure 3.5 Typical appearance of compressive (left) damage and tensile (right) damage.

Also, there were extensive areas of diffuse damage which would have been undetected by a light microscopes Figures 3.6 and 3.7.
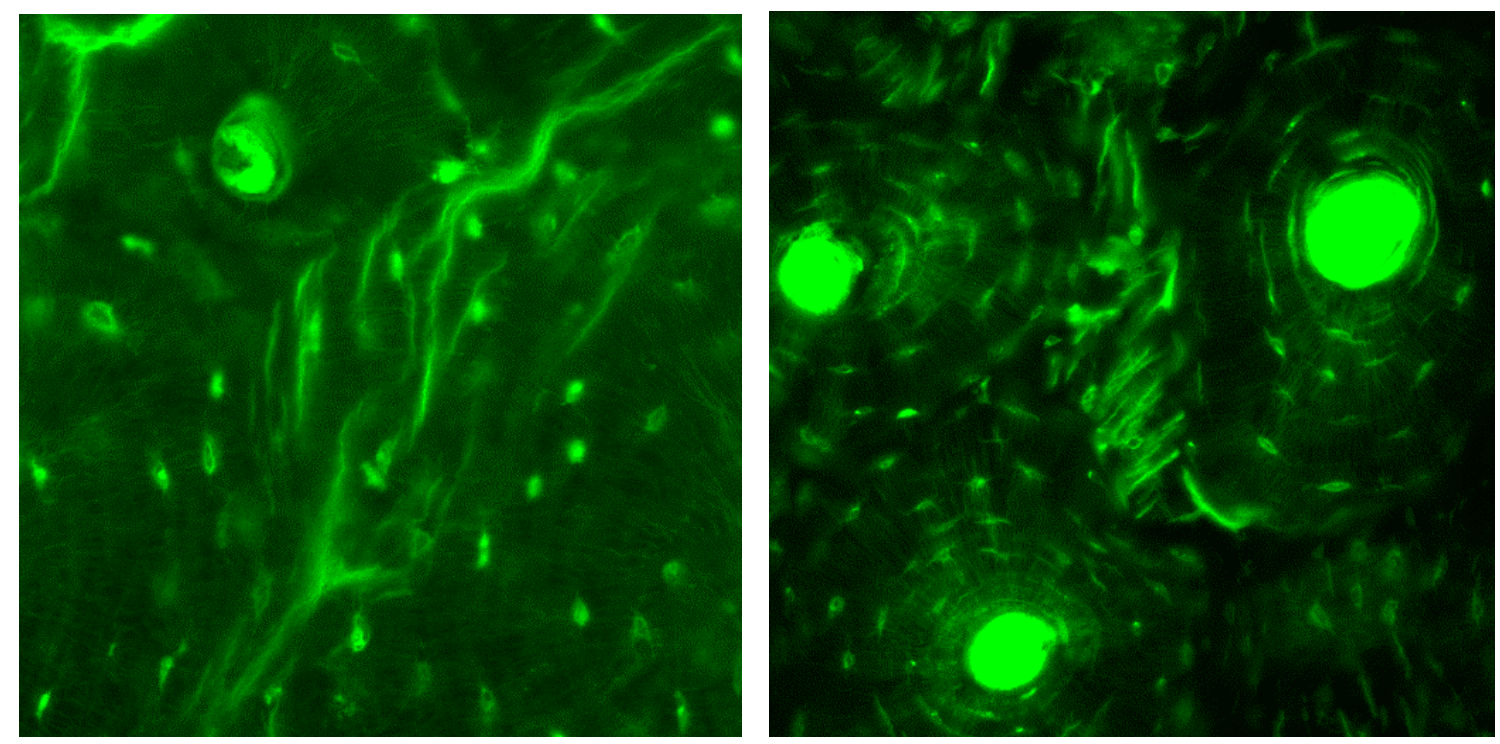

Figure 3.6. Areas of diffuse damage in the matrix region. 
Figure 3.8 demonstrates the ability of confocal microscope to identify the microscopical features undetected by a regular light microscope. The image is a superposition of two which was thresholded to highlight the advantages of one versus the ather.
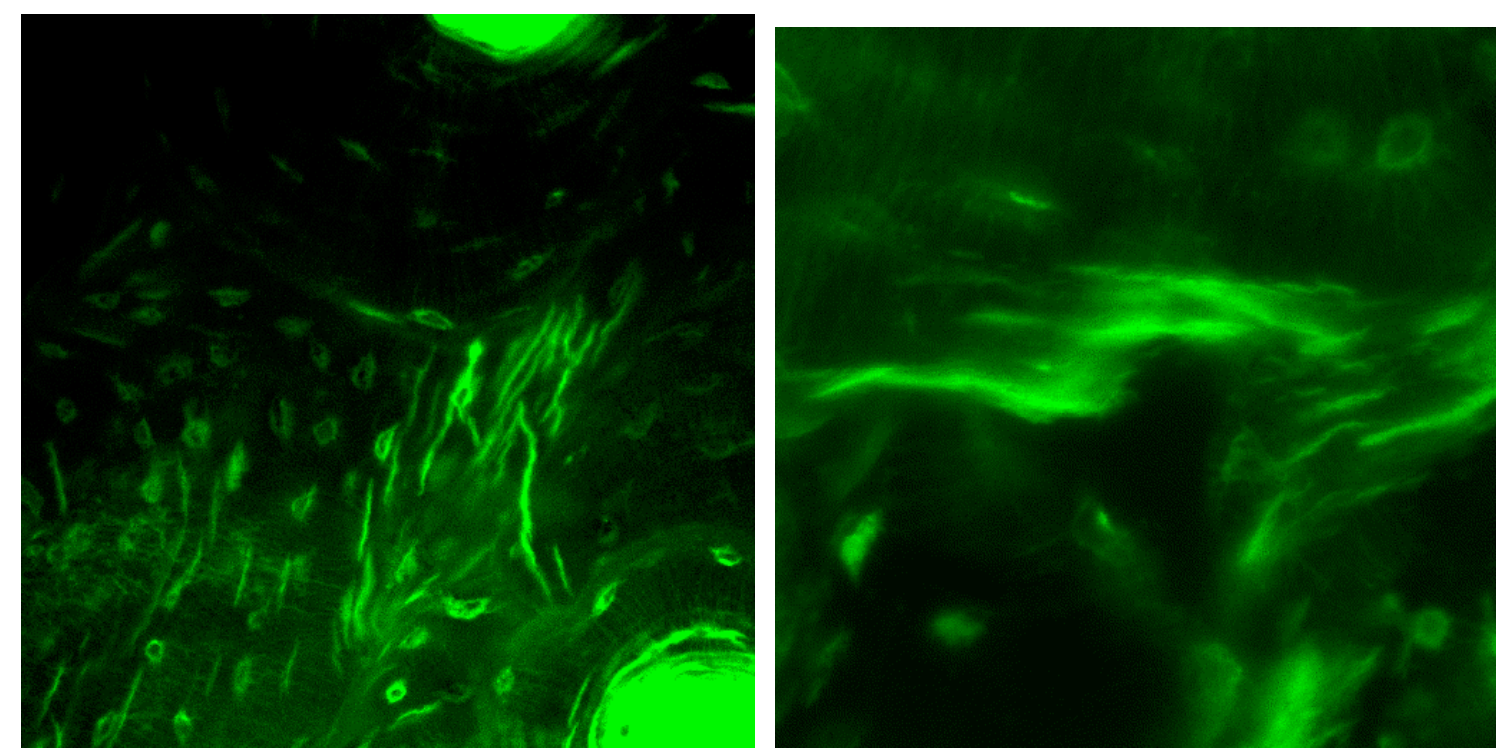

Figure 3.7. Areas of diffuse damage.

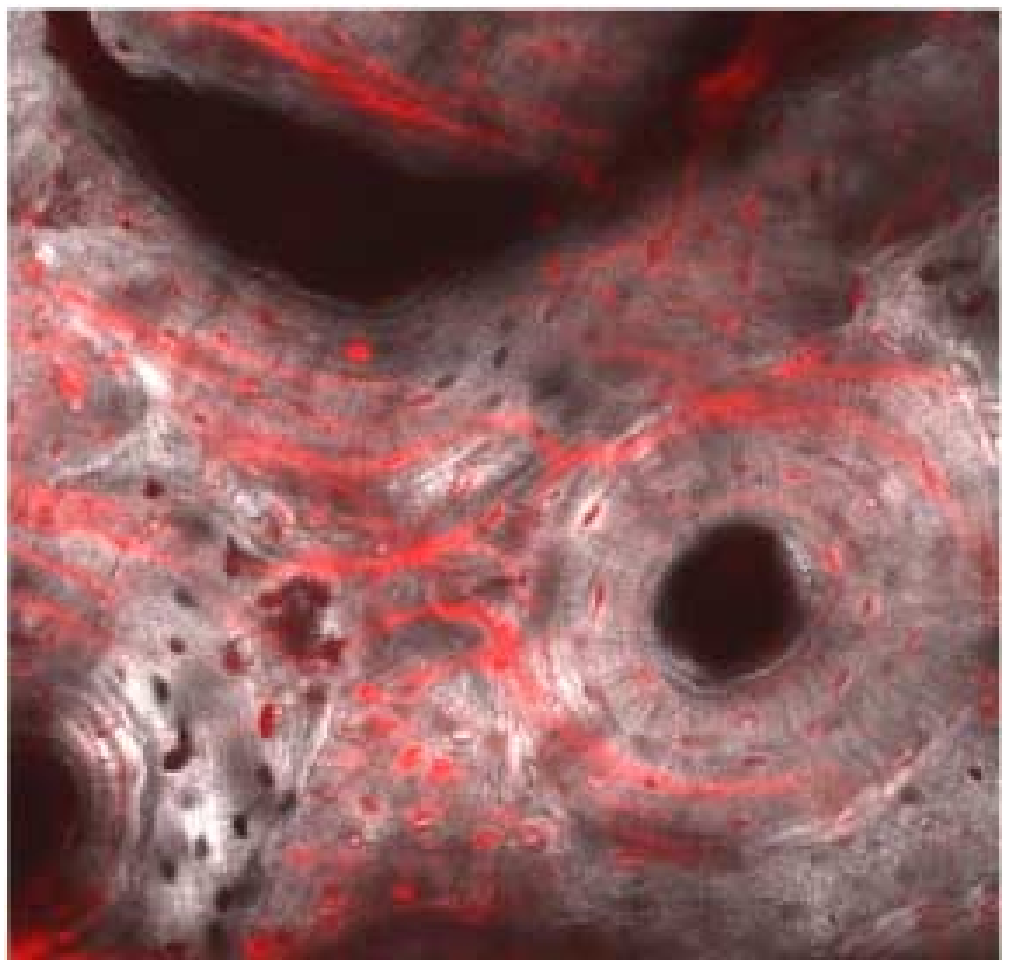

Figure 3.8 A superimposed image obtained by LSCM and light microscope. 

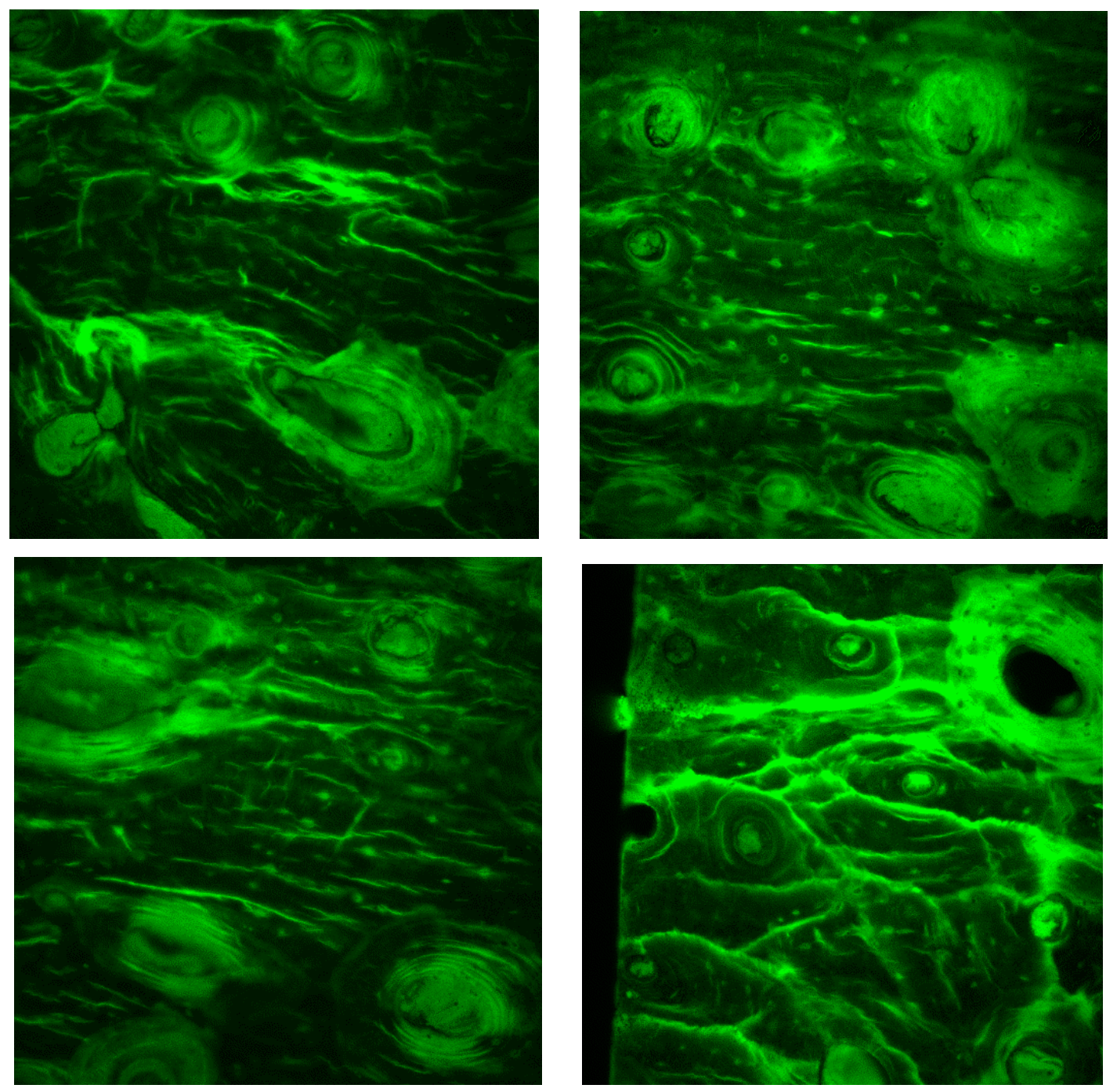

Figure 3.9 Confocal micrograph of sections from an entire cross-section of specimen from $\mathrm{G} 2$.

Gross observations of the failure patterns revealed that G1 specimens failed under mixed mode and G2 specimens failed in shear (Figure 3.10). This behavior resembles the one of short fiber reinforced composites (Lin and Lai, 1993). In the tensile regions failure occurs due to the rupture of fibers (osteons) and their pullout. As a result the catastrophic crack propagates perpendicular to the direction of tensile forces. And since it is known that bone is very strong in compression, the catastrophic crack changes its direction once it reaches the plane of neutral axes, where the dominant forces are the shear forces. Shear forces tend to deflect the propagation path of the crack along the weak interfaces of bone such as interlammellar spaces and osteonal cement lines. This scenario leads to the mixed 
mode failure. In the case of a short aspect ratio the overall scenario becomes different. In this case shear forces start to dominate throughout the depth of the beam. Hence, the path of the propagating crack is along the trajectories of the principal stress, which form a $45^{\circ}$ angle with the neutral axis. Therefore, this explains the curved and angled failure contours observed for specimens from $\mathrm{G} 2$.
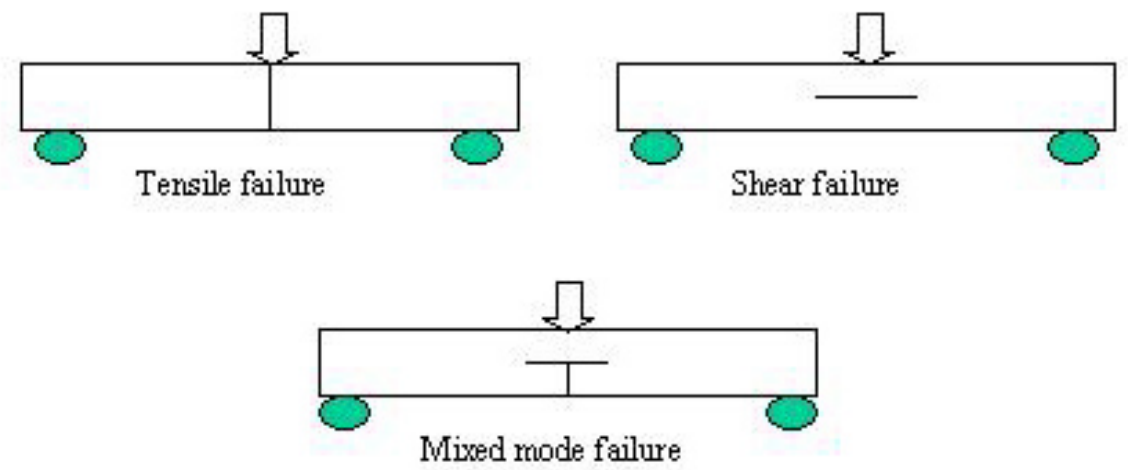

Figure 3.10 Schematic diagram of three major modes of failure under 3-point bending conditions.

\subsubsection{Damage Evolution}

Results of stress strain behavior would be described on typical plots from a single specimen from each group. Figures 3.11 and 3.12 represent stress-strain history of specimens RDL02 and RMP04 from G1 and G2, respectively. For each load ramp linear portions were identified and linear regression was performed to calculate the stiffness of the specimen at the corresponding cycle. In addition, total and residual strains were identified for each ramp. Afterwards, the damage parameter D, defined as the ratio of the stiffness of Nth cycle to the stiffness of the initial cycle $\left(1-\mathrm{D}=\mathrm{E}_{\mathrm{N}} / \mathrm{E}_{\mathrm{O}}\right)$, was determined for each consecutive cycle. Figures 3.13 and 3.14 represent the resulting relationships of G1 and G2, respectively, as a function of total strain. According to the principle of equivalent strain, the expected relationship should be linear. Our results indicate that the relationship is quadratic, which is in accordance to the principle of strain energy equivalence. It was demonstrated that this behavior characterizes damage accumulation in anisotropic materials (Chow and Wang, 1987). It also incorporates the Poisson effects and damage induced anisotropy in the material, which are not described by the principle of equivalent strain. 


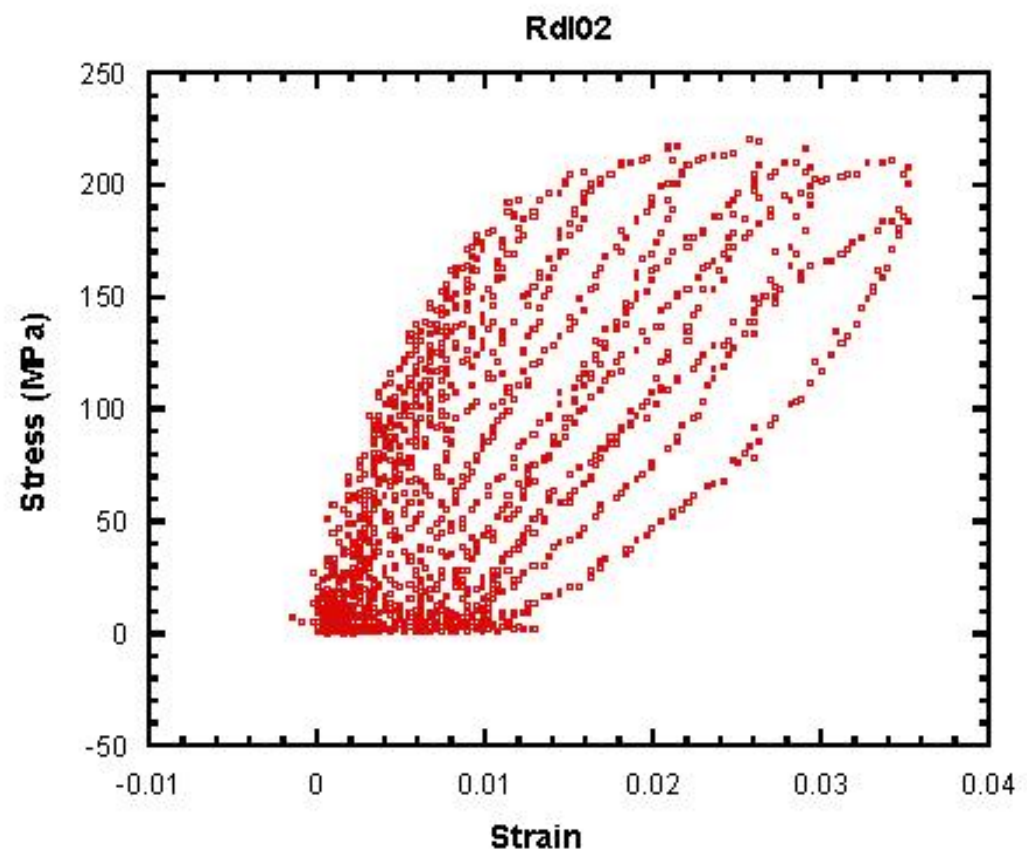

Figure 3.11. Stress strain history of specimen RDL02 from G1.

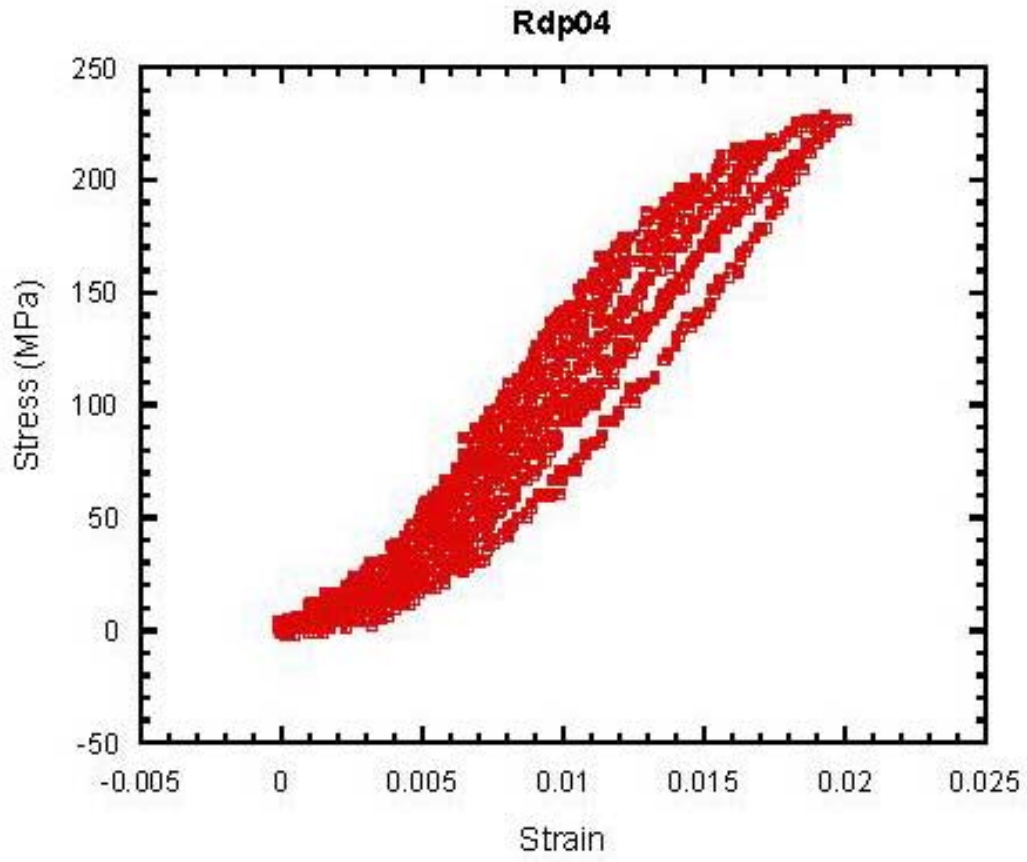

Figure 3.12 Stress-strain history of specimen RDP04 from G2. 


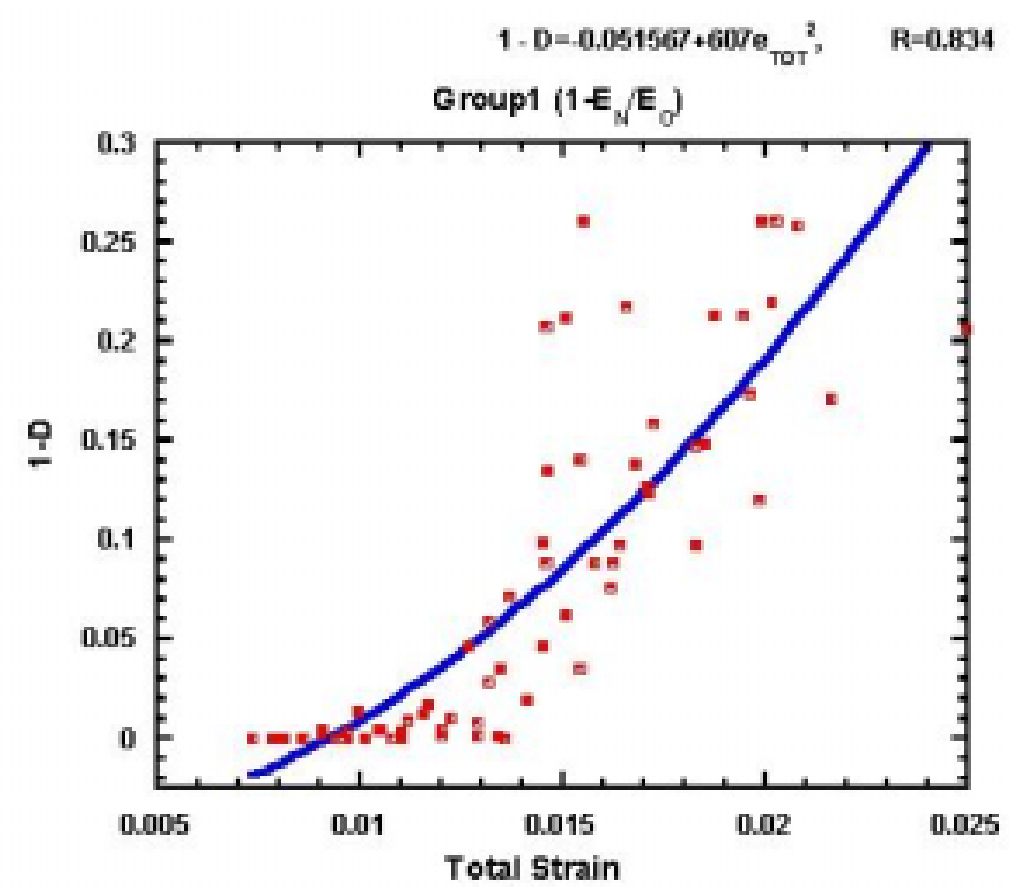

Figure 3.13. Damage Evolution of specimens from G1.

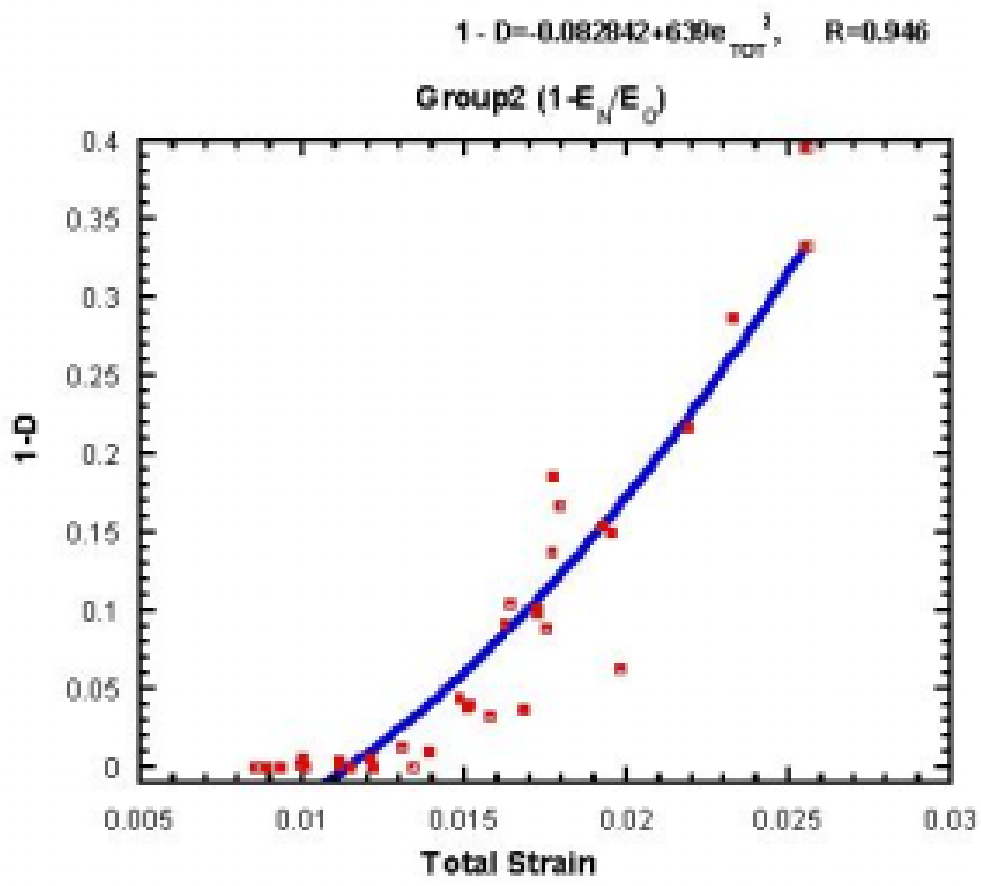

Figure 3.14 Damage Evolution of specimens from G2. 
The resulting equations for damage evolution are given

$$
1-D=-0.051567+607 \varepsilon^{2} \quad \text { for } \mathrm{G} 1
$$

and

$$
1-D=-0.082842+639 \varepsilon^{2} \quad \text { for } \mathrm{G} 2
$$

Further investigation of the behavior of unrecovered residual strains revealed a very interesting observation. It appeared that there was a consistent relationship between the total strain of each cycle and the residual strain at the end of the same cycle. The relationship appeared to be constant. Namely, it was found that the portion of strain that was not recovered upon the removal of the load was related to the maximal strain of the cycle by a constant. And more interestingly, the constant was the same for the consecutive cycles. Figures 3.15 and 3.16 demonstrate the relationship between the ratio of residual and total strains and the total strains for all the specimens from G1 and G2, respectively.

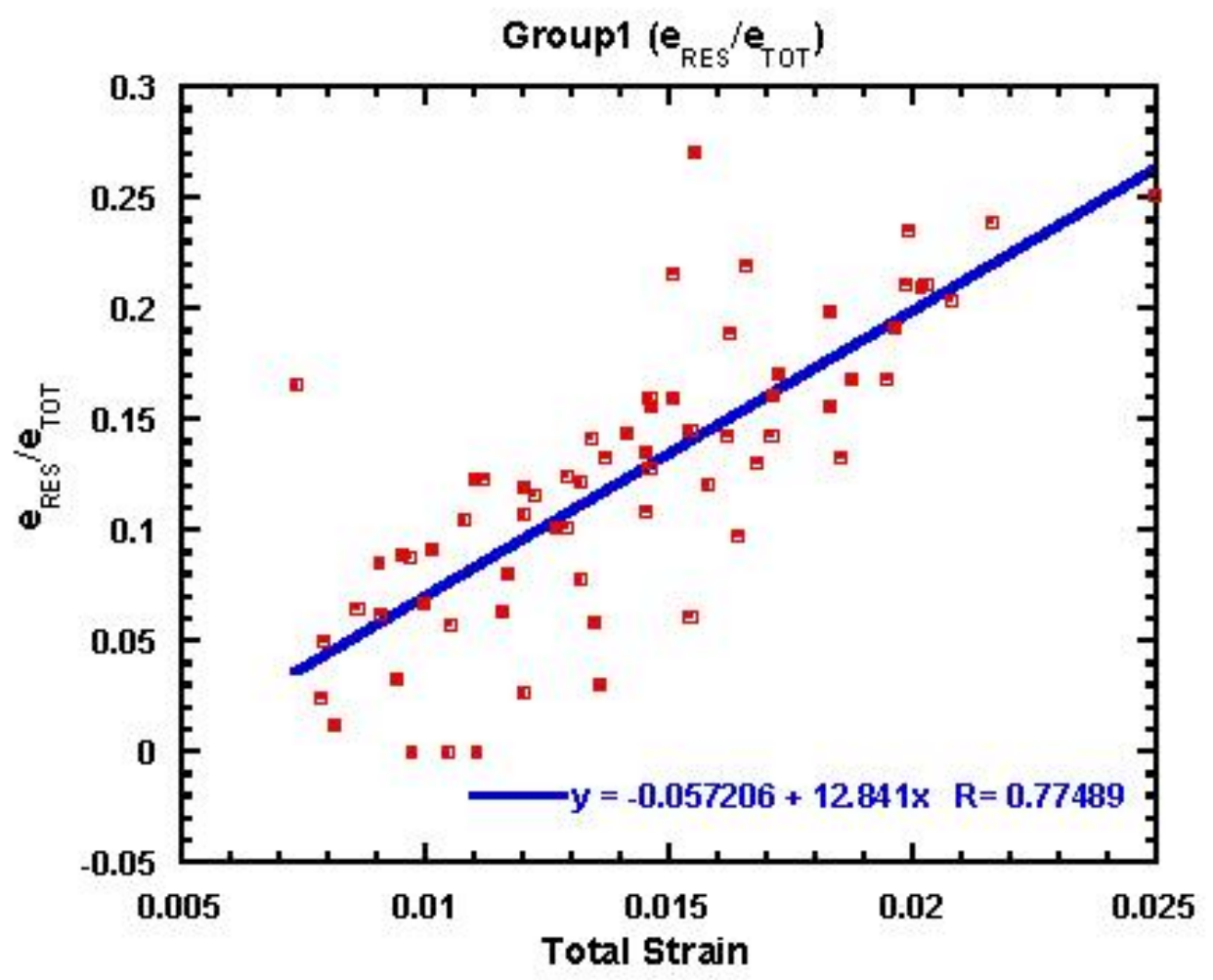

Figure 3.15. The observed relationship between the ratio of residual and total strains as a function of maximal strains for G1 $(p<0.0001)$. 


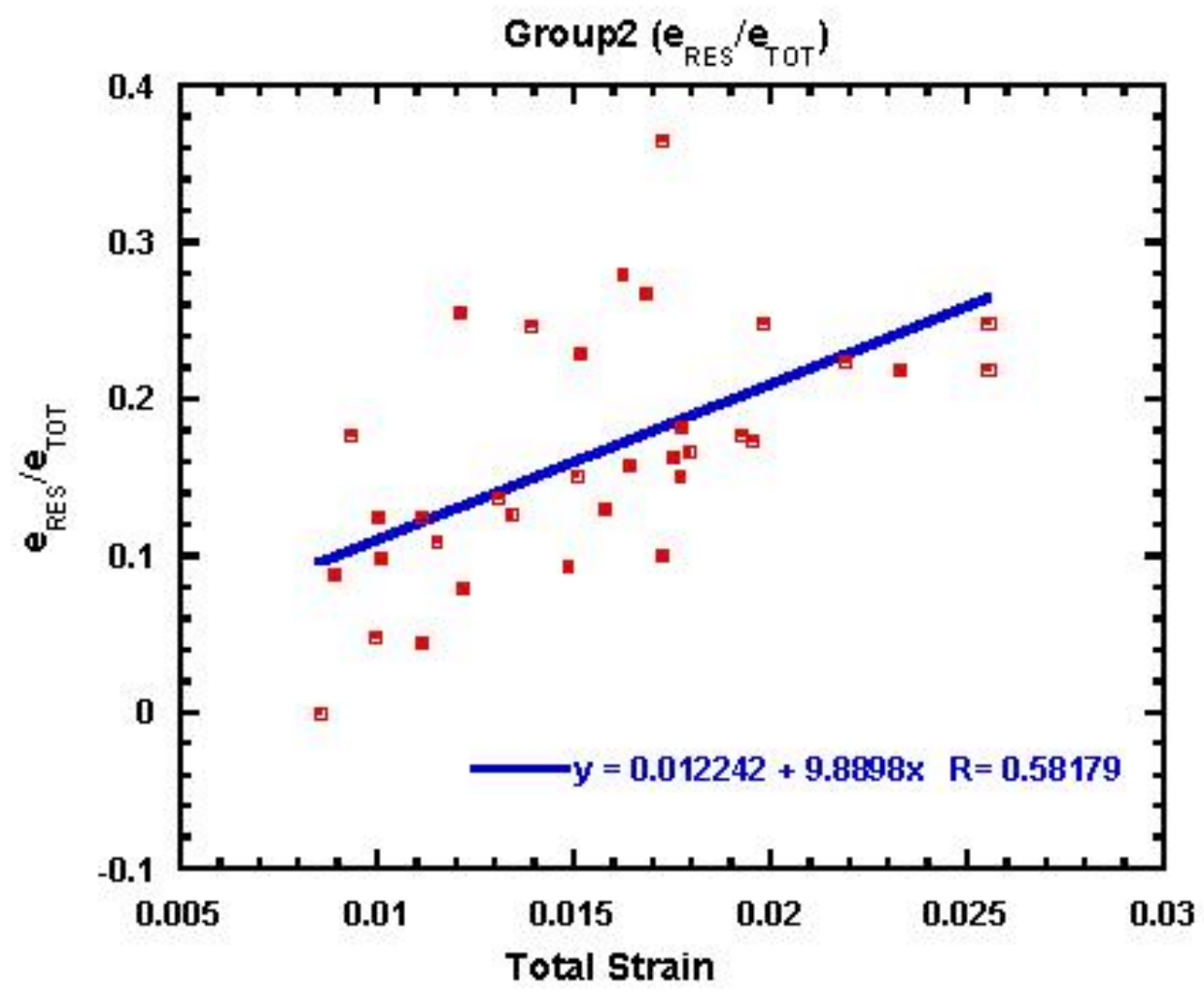

Figure 3.16. The observed relationship between the ratio of residual and total strains as a function of maximal strains for $\mathrm{G} 2$ ( $\mathrm{p}=0.0003)$.

In order to determine whether those relationships are statistically significant, statistical analysis of the linear fit was performed using a statistical software package JMP. The results showed that the relationships obtained for both Groups were statistically significant (Figures 3.17 and 3.18). Hence the obtained relationships are statistically significant and could be readily utilized. Those relationships are given

$$
\varepsilon_{p}=-0.057206 \varepsilon+12.841 \varepsilon^{2} \quad \text { for } \mathrm{G} 1
$$

and

$$
\begin{array}{ll}
\varepsilon_{p}=-0.012242 \varepsilon+9.8898 \varepsilon^{2} & \text { for } \mathrm{G} 2
\end{array}
$$




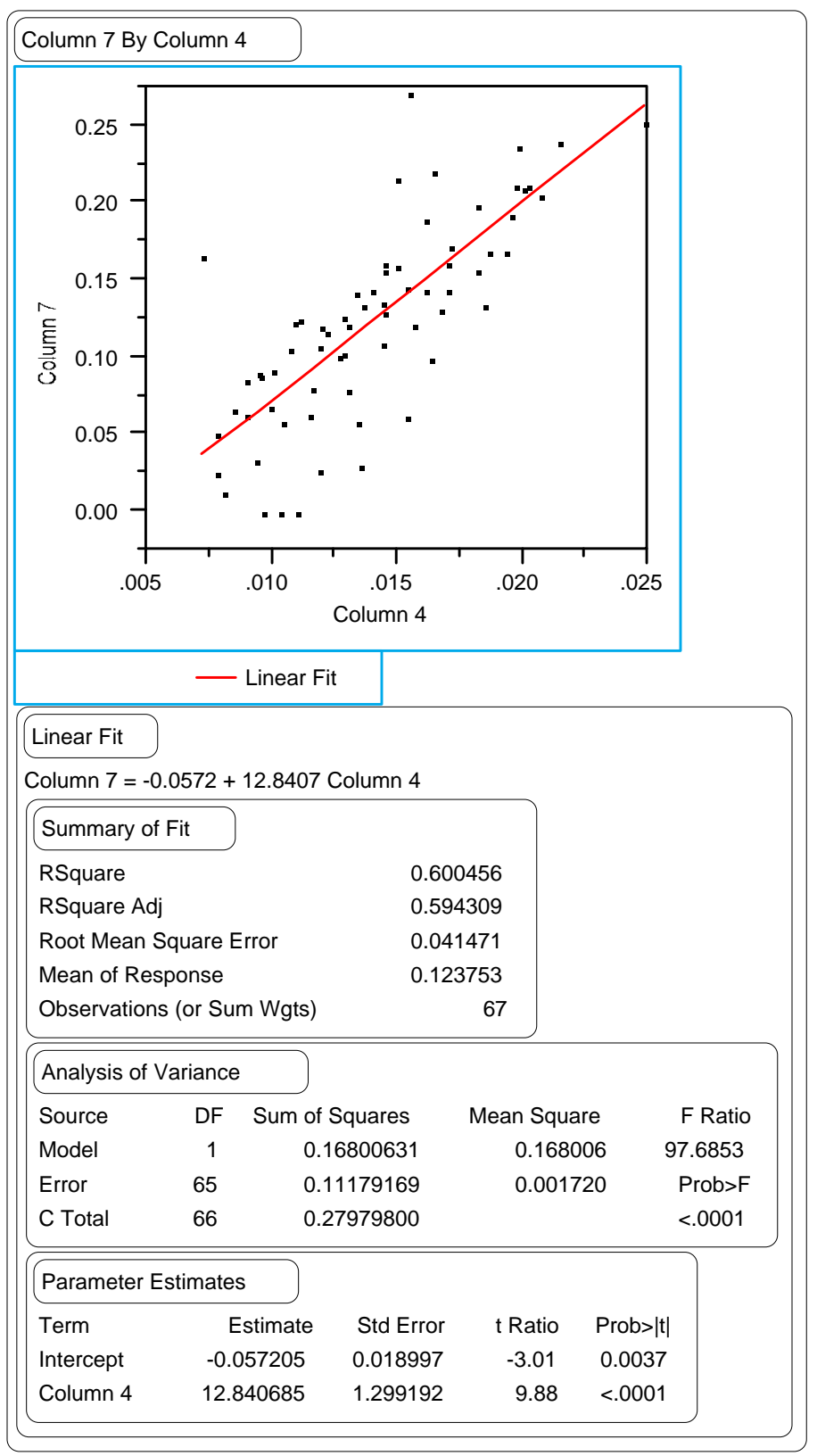

Figure 3.17. Analysis of variance (ANOVA) have demonstrated that the linear fit is statistically significant fit of data given in Figure 3.15 for the G1. 


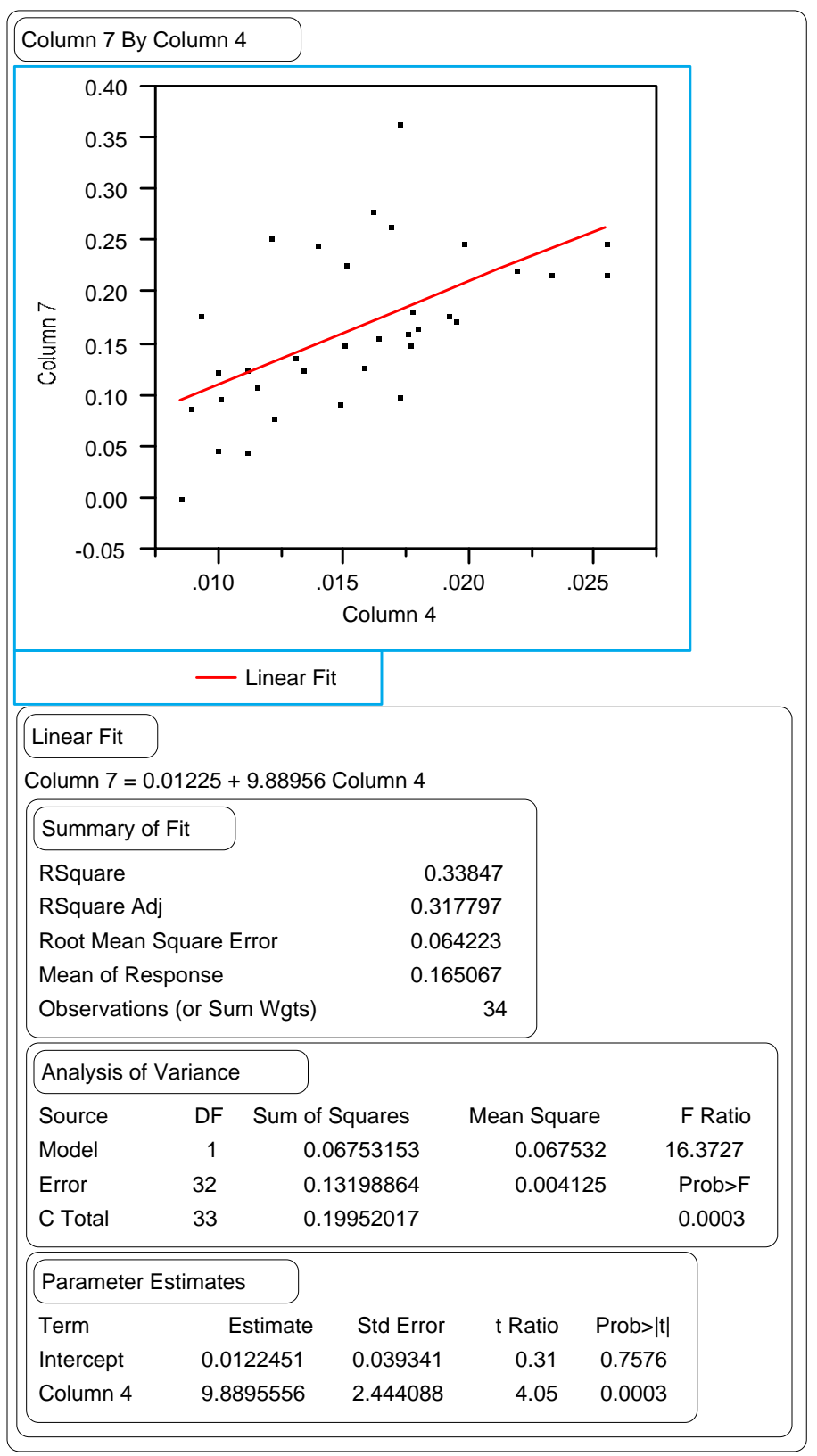

Figure 3.18. Analysis of variance (ANOVA) have demonstrated that the linear fit is statistically significant fit of data given in Figure 3.16 for the G2. 


\section{CHAPTER 4}

\section{CONTINUUM DAMAGE MODEL FOR HUMAN CORTICAL BONE}

\subsection{Introduction}

Since the pioneering work of Kachanov (1958) and Robotnov (1963) the Continuum Damage Mechanics (CDM) is a successfully developing branch of solid mechanics, which interlinks the experiences in continuum mechanics, fracture mechanics, materials science, physics of solids, etc. The fundamental problem of the solid mechanics of damaging materials is the characterization of their thermomechanical response. Two general approaches in addressing this problem are the Micromechanical approach and CDM.

Micromechanical approach, in general, attempts to predict the macroscale thermomechanical response of heterogeneous materials based on mesostructural models

of a representative volume element (RVE) within the material. A RVE may be defined as the minimum material volume that contains a sufficient number of damage entities to provide a statistically homogeneous representation of the mesostructure. Micromechanical models have the distinct advantage of being able to capture structural details at the microscale and mesoscale, and to allow formulation of kinetic equations for damage evolution based on the actual physical processes involved.

$\mathrm{CDM}$ provides an alternative framework for the development of constitutive equations and damage evolution equations for cracked solids. CDM is based on the thermodynamics of irreversible processes (Kestin and Rice, 1970; Rice, 1971), internal state variable theory (Coleman and Gurtin, 1967), and relevant physical considerations (assumptions of distributed damage variable, homogenization concepts, definition of damage variable, formulation of kinetic laws for damage evolution, etc.). A solid that is highly heterogeneous at the mesoscale is considered an effective homogeneous continuum at the macroscale. Macroscopic damage variables are judiciously selected that reflect the effects of mesostructural level irreversible processes on macroscale material behavior. Such damage descriptors may be obtained through a low order homogenization (spatial average) of individual damage entities, implying that damage is uniformly distributed throughout the RVE. The fact that the theory refers to a homogeneous continuum, however, presents a serious obstacle in the development of damage evolution 
laws that incorporate effects of the heterogeneity of the solid at the mesoscale. Whereas, effective moduli are somewhat insensitive to the distribution of microcracks, damage evolution is highly dependent on the local fluctuations in crack array geometry (Kachanov, 1992).

The effective moduli of a damaged solid largely depend on the average distribution of sizes, orientations, and spatial positions of defects within the RVE. When damage consists of weakly interacting microcracks, estimates of the effective moduli of solids with stationary microcracks may be obtained using the self-consistent method (Budiansky and O’Connel, 1976), generalized self-consistent method (Hoenig, 1979), differential self-consistent scheme (Hashin, 1988), Mori-Tanaka method (Mori and Tanaka, 1973) or other such "effective medium" approach. Damage evolution, however, depends on the extreme values of the defect distribution (i.e., largest defect size, minimum nearest neighbor distance between flaws, etc.). Hence, damage evolution, unlike effective stiffness, is largely governed by the local fluctuations of the stress field that, in turn, depends on the crack geometry.

Development of damage evolution laws is critical to the development of a thermomechanical constitutive theory for damaged materials. Given the large scale numerical calculations generally required for micromechanical analysis of materials with damage, perhaps the most practical vehicle for the development of a general thermomechanical constitutive theory for damaged solids lies within the framework of CDM using internal variables.

\subsection{Fundamentals of Classical Thermodynamics}

The fundamental assumption of classical thermodynamics is that the state of a phase can be uniquely specified by a set of independent thermomechanical variables. Which means that it is assumed that the state of phase is independent of the previous history of processes.

\subsubsection{First Law of Thermodynamics (Conservation of Energy)}

The first principle of thermodynamics states that, if thermally insulated system can be taken from a state I to a state II by alternative paths, the work done on the system has the same value for every such (adiabatic) path. From this it is deduced that there 
exists a single-valued function of the state of a system, called its energy and that the energy balance is satisfied:

$$
\frac{d}{d t}(E+K)=P+Q
$$

where $Q$ is the rate at which heat is received by a closed region within the body and consists of the heat generated within this region by external forces and heat received by conduction through the boundary of the region, $E$ is the internal energy, $K$ is the kinetic energy and $P$ is the power of external forces. This principle infers the existence of an internal energy function and that the work done in an adiabatic process in going from one state to another is independent of path.

In continuum mechanics it is more convenient to express the equation of energy balance using the definitions of mass density $\rho$, the specific energy density e, volumetric density of the internal heat production $r$ and heat flux vector $\vec{q}$ :

$$
\rho \frac{d e}{d t}=\sigma_{i j} \dot{\varepsilon}_{i j}+r-q_{i, i}
$$

\subsubsection{The Second Law of Thermodynamics}

The second principle of thermodynamics states that: There exist two single-valued

functions of state, $T$, called the absolute temperature, and $S$, called the entropy, with a following properties:

- $T$ is always positive and is a function of the empirical temperature only

- The entropy of a system is equal to the sum of entropies of its parts

- The entropy of a system can change in two distinct ways: by interaction with the surroundings and by changes taking place inside the system:

$$
d S=d_{e} S+d_{i} S
$$

where $d_{e} S$ denotes the part of the increase of entropy due to interaction with the surroundings, and $d_{i} S$ denotes the part of this increase due to changes taking place inside the system. So, if $d Q$ denotes the heat absorbed by the system from its surrounding, then

$$
d S=\frac{d Q}{T}
$$


If $d_{i} S=0$ then the process is said to be reversible, if $d_{i} S>0$ then the process is irreversible and $d_{i} S<0$ never occurs in nature. This statement connects the theory with the nature by stating the direction of motion of events in the world. According to the proverb, "Time is an arrow", the second law endows the arrow of time.

Equation (4.3) could be written in the local form via application of divergence theorem resulting in

$$
\rho \frac{d s}{d t}+\vec{\nabla} \cdot\left(\frac{\vec{q}}{T}\right)-\frac{r}{T} \geq 0
$$

This inequality is known as Clausius-Duhem inequality, where the first term $\frac{d s}{d t}$ represents the change of total specific entropy, $\frac{r}{T}$ is the entropy influx due to the distributed heat supply and $\frac{1}{\rho} \vec{\nabla} \cdot\left(\frac{\vec{q}}{T}\right)$ the entropy influx due to heat influx.

Legendre transformations can be used to obtain other functions of state. If we define Helmholtz free energy per unit reference volume as

$$
\Psi=e-T s
$$

and Gibbs free energy as

$$
\Phi=\Psi-\frac{1}{\rho} \sigma_{i j} \varepsilon_{i j}=e-T s-\frac{1}{\rho} \sigma_{i j} \varepsilon_{i j}
$$

and taking material derivatives of those thermodynamic potentials we can rewrite the Clausius-Duhem inequality as:

$$
\sigma_{i j}: \dot{\varepsilon}_{i j}-\rho(\dot{\Psi}+s \dot{T})-\frac{q_{i} T_{, i}}{T} \geq 0
$$

and

$$
-\dot{\Phi}-\varepsilon_{i j} \dot{\sigma}_{i j}-s \dot{T}-\frac{q_{i} T_{, i}}{T} \geq 0
$$

\subsubsection{Thermodynamics of irreversible processes}

In seeking a mathematical framework within which to develop constitutive equations, a general approach should be followed. Namely, the Clausius-Duhem 
inequality, which is taken from basic laws of physics, should be introduced into the equations of continuum mechanics by the restrictions it places on the constitutive equations. The constitutive equations themselves relate a set of independent variables, usually strain, temperature, and temperature gradient, to a set of dependent variables, usually stress, free energy, and entropy. The restrictions placed on these relationships by the Clausius-Duhem inequality ensures that the second law of thermodynamics is satisfied for all admissible processes.

The knowledge that the relationship between the independent and dependent variables must satisfy the Clausius-Duhem inequality does little to narrow the possible choices for constitutive equations. Fortunately, fairly general mathematical frameworks have been suggested as being appropriate methods for constitutive equation development (Coleman and Gurtin, 1967). The description of more complicated processes during which the microstructure can be irreversibly rearranged requires the introduction of additional kinematic variables. Since the energy must be dissipated during the irreversible rearrangements of the microstructure the entropy must depend on these additional kinematic variables or alternatively known as internal variables. Each internal variable describes a particular dissipative mechanism of the microstructural rearrangement. Equilibrium is induced by a set of fictitious thermodynamic forces, conjugate to the selected internal variables. More specifically, the thermodynamic forces act on the microstructural rearrangement described by the conjugate internal variable. Mathematically, this approximation serves to replace the memory functional representation of constitutive laws by the differential equations.

The selection of internal variables should involve a transition from an ensemble of micro-systems with a heterogeneous and piece-wise continuous microstructure to an idealized, continuous medium. However, before giving their definition, a choice must be made with regard to the nature of the variables. In this choice lie both the weakness and the readiness of the method of phenomenological thermodynamics. This transition, referred to as homogenization, is possible only if the micro-systems are large enough to be statistical homogeneous and simultaneously small enough to be able to consider the traction on its boundary constant. Viscoelasticity, damage and plasticity are forms of irreversible thermodynamic processes. 
Mathematically, the formulation of the thermodynamics of irreversible processes follows the following algorithm. First, state variables must be defined and their relationship derived via postulation of the existence of a thermodynamic potential (Helmholtz free energy) leading to the derivation of the state laws. Then the dissipation laws of the internal state variables must be derived via postulation of the existence of dissipation potential (Gibbs free energy) leading to the evolution laws of internal variables.

In general definition of the thermodynamic forces associated with the internal variables is given by:

$$
A_{k}=\rho\left(\frac{\partial \Psi}{\partial V_{k}}\right)
$$

where conjugate forces $A_{k}$ associated with internal variables $V_{k}$ by specification of the thermodynamic potential $\Psi\left(\ldots V_{k}\right)$. It could also be shown that

$$
\begin{aligned}
& \sigma=\rho\left(\frac{\partial \Psi}{\partial \varepsilon^{e}}\right) \\
& s=-\frac{\partial \Psi}{\partial T}
\end{aligned}
$$

Equations (4.9)-(4.11) constitute the state laws.

\subsection{Review of pertinent literature on CDM theory}

Historically, Kachanov (1958) provided a basis for the Rabotnov's effective stress concept (1969), which has led to foundation of the development of phenomenological models, which utilized the concept of a quasicontinuum approximation of the damaged material. They argued that in the presence of damage represented, as the surface density of discontinuities in the material would lead to the reduction of an area of resistance, thus leading to some effective area of resistance $\widetilde{S}=S-S_{D}=S(1-D)$. Then they proceeded to define the effective stress that efficiently resists forces by $\widetilde{\sigma}=\sigma S / \widetilde{S}=\sigma /(1-D)$. In 1975 Lemaitre and Chaboche introduced the concept of effective stiffness, which was 
based on the principle of strain equivalence. They assumed that the deformation of a material is only affected by damage in form of effective stress (Figure 4.1).

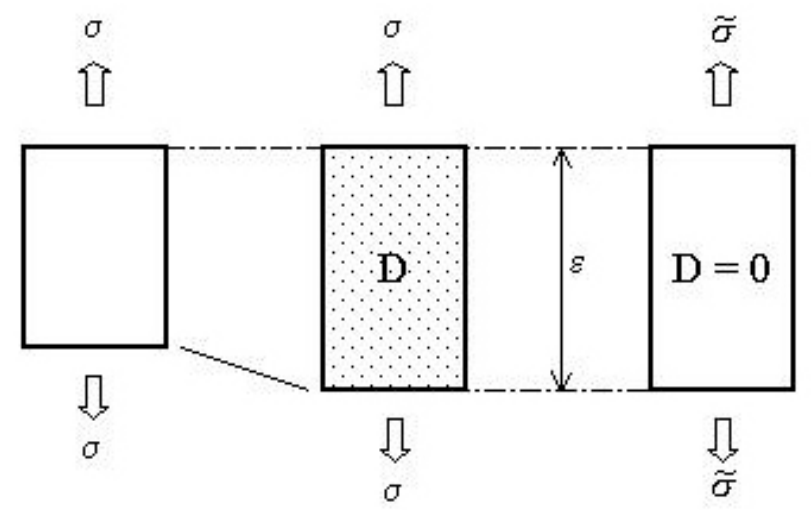

Figure 4.1. Effective stress and the equivalence of strain.

This, in turn, had meant that $\widetilde{\sigma}=\sigma /(1-D)=E \varepsilon$ or $\sigma=E(1-D) \varepsilon$, which lead that $E(1-D)=\widetilde{E}$ could be viewed as effective stiffness or the stiffness of the damaged material. This approach assumes that all the different behaviors such as elastic, plastic or viscoplastic are affected in the same way by the surface density of the damage effects. Hence, it allowed the establishment of a coherent and efficient formalism.

Based on this interpretation of damage, many researchers focused on generalizing the 1-D constitutive equation of damaged material to anisotropic damage states induced by a three-dimensional distribution of defects. Such research includes scalar damage variable (Lemaitre, 1985), vector variables (Davison and Stevens, 1973; Krajcinovic and Fonseka, 1981; Krajcinovic, 1985), second order tensors (Murakami and Ohno, 1981) and fourth order tensors (Simo and Ju, 1987; Chow and Wang, 1987; Yazdani, 1993).

There has been a considerable amount of development in systematic coupled damage-constitutive theories of engineering materials. Those include, models for investigation of the influence of both isotropic and anisotropic damage evolution on the constitutive equations of brittle, creep-plastic solids, creep-viscoplastic, fiber reinforced composites, fiber reinforced plastics and other materials. These include numerous studies by J. Lemaitre, J.L. Chaboche, D. Krajcinovic, C.L. Chow, G.Z. Voyiadjis, S. Murakami, J.C. Simo and many other authors. 


\subsection{Continuum Damage Model for human cortical bone}

\subsubsection{Introduction}

Fondrk and co-workers, who have conducted extensive studies of nonlinear behavior of cortical bone, concluded that bone is a damaging viscoelastic material. That is, it behaves as a linear viscoelastic material at stress levels below a threshold level and it demonstrates viscoelastic creeping behavior above the threshold stress. They also demonstrated that series of loading-unloading cycles with a loading beyond the threshold value of stress causes stiffness degradation in addition to creep induced nonlinear strains, which were eventually partially recovered leading to permanent strains. This indicates that bone undergoes both damage and permanent deformation.

In order to model such a behavior a thermodynamic framework is necessary that would model continuum damage behavior of viscoelastic materials and would account for the possibility of a permanent deformation component in the behavior. The majority of CDM models were developed to model time-independent deformation processes, such as elastic-brittle or elastic-plastic behavior. Only few models have addressed the issue of modeling continuum damage behavior of viscoelastic materials.

Coleman and Noll (1963) proposed a general approach for the development of constitutive equations by introducing Clausius-Duhem inequality into the equations of continuum mechanics. They state that in phenomenological theories of the dynamical behavior of continua there are several ways of accounting for the dissipative effects which, in addition to heat conduction, accompany deformation. The oldest and simplest way is to introduce a viscous stress which depends on the rate of strain, as is done in the Navier-Stokes theory of linearly viscous fluids (Coleman and Mizel, 1964). Another description of dissipation assumes the entire past history of strain influences the stress in a manner compatible with the principle of fading memory (Coleman, 1964). A third approach is to postulate the existence of internal state variables which influence the free energy and whose rate of change is governed by differential equations in which strain appears (Coleman and Gurtin, 1967).

Schapery $(1980,1981,1984)$ in a series of papers proposed a basic formulatio for viscoelastic materials that included effects of damage. Based on those studies and on the existing concepts of the thermodynamic theory of viscoelastic materials (Biot, 1954, 
1955), Weitsmann $(1988$, 1998) introduced a viscoelastic model utilizing the methodology of continuum damage mechanics. These ideas and approaches would be utilized in the further development of CDM model for cortical bone.

\subsubsection{General Thermodynamic Considerations}

Coleman and Gurtin (1967) introduced the constitutive equations for internal state variable in the form

$$
\begin{aligned}
& \Psi=\bar{\Psi}(\varepsilon, T, g, \alpha) \\
& s=\bar{s}(\varepsilon, T, g, \alpha) \\
& \sigma=\bar{\sigma}(\varepsilon, T, g, \alpha) \\
& q=\bar{q}(\varepsilon, T, g, \alpha) \\
& \dot{\alpha}=f(\varepsilon, T, g, \alpha)
\end{aligned}
$$

where $\Psi$ is the specific Helmholtz free energy, $s$ is the specific entropy, $\sigma$ is stress, $q$ is the heat flux, $\varepsilon$ is strain, $T$ is temperature, $g=\frac{\partial T}{\partial x}$ is the temperature gradient, $\alpha$ is the set of internal state variables and bars correspond to the respond functions.

Those are $4+n$ one-dimensional equations, where $n$ depends on the number of internal state variables. And together with an equilibrium equation:

$$
\frac{\partial \sigma}{\partial x}=-b
$$

a kinematic equation:

$$
\varepsilon=-\frac{\partial u}{\partial x}
$$

and the energy equation:

$$
\dot{\Psi}+\dot{T} s+T \dot{s}-\frac{1}{\rho}\left[\sigma \dot{\varepsilon}-\left(\frac{\partial q}{\partial x}\right)\right]=r
$$

and the relationship $g=\frac{\partial T}{\partial x}$, there are total of $8+\mathrm{n}$ equations and $10+\mathrm{n}$ variables $(\sigma, b, \varepsilon$, $u, \Psi, T, s, q, g, r$ and $\alpha$ ), where $r$ is the volumetrically distributed heat supply, $b$ is the body force and $u$ is the $x$ displacement. 
They defined a thermodynamic process as a set of the $8+\mathrm{n}$ functions $(u, \sigma, b, \Psi$, $T, s, q, r$ and $\alpha$ ) that satisfy the equilibrium equation (4.13) and energy equation (4.15). A thermodynamic process can be specified by prescribing the functions $u, \sigma, \Psi, q, s, T$ and $\alpha$ as functions of $x$ and $t$, and then using equations (4.13) and (4.15) determine $r$ and b. They defined an admissible thermodynamic process as a thermodynamic process that is compatible with equations (4.12). As a consequence, an admissible thermodynamic process can be specified by prescribing the functions $u$ and $T$. For instance, $u$ can be used with equation (4.14) to calculate $\varepsilon ; g$ can be calculated from $T ; \varepsilon, T, g$ and an initial value of $\alpha$ can then be used in equation (4.12e) to determine $\alpha$; equations (4.12a-d) can then be used to determine $\Psi, s, \sigma$, and $q$; and equations (4.13) and (4.15) can be used to determine $r$ and $b$. In summary, an admissible thermodynamic process can be specified by an arbitrary choice of $u$ and $T$ as functions of $x$ and $t$.

The Clausius-Duhem inequality states that the internal production of entropy, $\gamma$ is non-negative:

$$
\gamma=\dot{s}-\frac{r}{T}+\frac{1}{\rho} \frac{\partial}{\partial x}\left(\frac{q}{T}\right) \geq 0
$$

The restriction that this inequality places on the response functions by substituting the time derivatives of equations (4.12)

$$
\dot{\Psi}=\frac{\partial \bar{\Psi}}{\partial \varepsilon} \dot{\varepsilon}+\frac{\partial \bar{\Psi}}{\partial T} \dot{T}+\frac{\partial \bar{\Psi}}{\partial g} \dot{g}+\frac{\partial \bar{\Psi}}{\partial \alpha} \dot{\alpha}
$$

into the energy equation (4.15) in order to eliminate $\frac{\partial \Psi}{\partial t}$ and then by substituting this modified version of energy equation into the Clausius-Duhem inequality (4.116) in order to eliminate $r$. As a result

$$
\gamma=\frac{1}{T}\left(\frac{1}{\rho} \sigma-\frac{\partial \bar{\Psi}}{\partial \varepsilon}\right) \dot{\varepsilon}-\frac{1}{T}\left(s+\frac{\partial \bar{\Psi}}{\partial T}\right) \dot{T}-\frac{1}{T} \frac{\partial \bar{\Psi}}{\partial g} \dot{g}-\frac{1}{T} \frac{\partial \bar{\Psi}}{\partial \alpha} f-\frac{1}{T^{2} \rho} g q \geq 0
$$

must be satisfied for all admissible thermodynamic process. They defined functions $u$ and $T$ at an arbitrary location and time (denoted as $x^{\prime}$ and $t_{0}$ ) and for an arbitrary point in the independent variable space (denoted $\varepsilon_{0}, T_{0}, g_{0}, \alpha_{0}$ ) to be:

$$
u=u_{0}+\left[\varepsilon_{0}+\left(t-t_{0}\right) A\right]\left(x-x^{\prime}\right)
$$

and 


$$
T=T_{0}+\left(t-t_{0}\right) B+\left[g_{0}+\left(t-t_{0}\right) C\right]\left(x-x^{\prime}\right)
$$

where $A, B$ and $C$ are arbitrary constants. And as it was mentioned earlier, prescribing $u$ and $T$ specifies an admissible thermodynamic process; consequently, this definition of $u$ and $T$ represents an arbitrary admissible thermodynamic process. These expressions can be differentiated, evaluated at $t_{0}$ and $x^{\prime}$ and substituted (along with the expressions for the response functions) into (4.18) to produce:

$$
\gamma=\left(\frac{\sigma}{\rho}-\frac{\partial \bar{\Psi}}{\partial \varepsilon}\right) A-\left(\bar{s}+\frac{\partial \bar{\Psi}}{\partial T}\right) B-\frac{\partial \bar{\Psi}}{\partial g} C-\frac{\partial \bar{\Psi}}{\partial \alpha} f-\frac{1}{T_{0} \rho} g_{0} \bar{q} \geq 0
$$

All the response functions are evaluated at $\varepsilon_{0}, T_{0}, g_{0}$ and $\alpha_{0}$. Since the choice of $A, B$ and $C$ was arbitrary this inequality can hold only if

$$
\begin{aligned}
& \bar{\sigma}=\rho \frac{\partial \bar{\Psi}}{\partial \varepsilon} \\
& \bar{s}=-\frac{\partial \bar{\Psi}}{\partial T} \\
& \frac{\partial \bar{\Psi}}{\partial g}=0 \\
& \frac{\partial \bar{\Psi}}{\partial \alpha} f+\frac{1}{\rho T_{0}} g_{0} \bar{q} \leq 0
\end{aligned}
$$

where the response functions are all evaluated at $\varepsilon_{0}, T_{0}, g_{0}$ and $\alpha_{0}$. However, since the choice of $x^{\prime}, t_{0}, \varepsilon_{0}, T_{0}, g_{0}$ and $\alpha_{0}$ was arbitrary, the relationships given in (4.19) must generally hold to preserve the Clausius-Duhem inequality. If $f$ is not a function of $g$ and $\bar{q}$ is not a function of $\alpha$, the last inequality can be separated into:

$$
\begin{aligned}
& \frac{\partial \bar{\Psi}}{\partial \alpha} f \leq 0 \\
& -\frac{1}{\rho T_{0}} g_{0} \bar{q} \leq 0
\end{aligned}
$$

which are referred as internal dissipation inequality and the heat conduction inequality. Defining a valid set of constitutive equations has been reduced to defining functions $\bar{\Psi}$ and $f$ that satisfy (4.20a). Once this is done, all of the other response functions can be determined from equations (4.19). 


\subsubsection{Viscoelasticity Coupled with Damage}

It was mentioned in section 3.1 that for accurate rheological characterization of material's stress-strain behavior it is very important to study it's recovery behavior upon the removal of external loads. Section 3.2 also summarized most of the work by the bone researchers that had studied this crucial aspect of material behavior. Fondrk and coworkers are the only ones to be known to carry out well-controlled study of bone's material characterization and it's damaging behavior. They have concluded that bone behaves as a linear viscoelastic material for stress levels below some threshold value of stress and it behaves as a damaging viscoelastic material beyond the threshold. To our knowledge Weitsmann (1988) and Abdel-Tawab and Weitsmann (1998) have developed the only continuum damage formalism for viscoelastic materials coupled with damage existing in the current literature. In this section we will adopt the general thermodynamic formalism introduced by those authors for the development of continuum damage model for human cortical bone.

We will start with the definition of the internal state variables (ISVs). Consider that $\alpha_{n}(r=1, \ldots, \mathrm{N})$ denote the $\mathrm{N}$ scalar valued internal state variables representing the internal degrees of freedom of molecular motion in a viscoelastic material. In addition, consider $\beta_{m}(s=1, \ldots, \mathrm{M})$ denote the $\mathrm{M}$ scalar valued internal state variables associated with permanent deformation. The ISVs associated with damage are taken in the form of a double symmetric fourth rank tensor $w_{p q r s}=w_{q p r s}=w_{p q s r}=w_{r s p q}$, which takes into account the damage-induced anisotropy of the material.

The energy dissipation inequality for a closed system with small strains can be written

$$
-\dot{\phi}-\varepsilon_{i j} \dot{\sigma}_{i j}-s \dot{T}-\frac{q_{i} T_{, i}}{T} \geq 0
$$

where $\phi$ is the Gibbs's free energy (energy per unit volume), $\varepsilon_{i j}$ is the strain tensor, $\sigma_{i j}$ is the Cauchy stress tensor, $s$ is the entropy per unit volume, $T$ is the temperature, $q_{i}$ is the heat flux vector, $T_{, i}$ is the temperature gradient.

Considering a dissipation potential (Gibbs free energy) in the form

$$
\phi=\phi\left(\sigma_{i j}, \alpha_{n}, \beta_{m}, w_{p q r s}, T\right)
$$

and proceeding in the manner described in Section 4.3.2. we can get that 


$$
\begin{aligned}
& \varepsilon_{i j}=-\frac{\partial \phi}{\partial \sigma_{i j}} \\
& S=-\frac{\partial \phi}{\partial T} \\
& \mathrm{~A}_{n}=-\frac{\partial \phi}{\partial \alpha_{n}} \\
& \mathrm{~B}_{m}=-\frac{\partial \phi}{\partial \beta_{m}} \\
& \Omega_{p q r s}=-\frac{\partial \phi}{\partial w_{p q r s}}
\end{aligned}
$$

and

$$
\mathrm{A}_{n} \dot{\alpha}_{n}+\mathrm{B}_{m} \dot{\beta}_{m}+\Omega_{p q r s} \dot{w}_{p q r s}-\frac{h_{i} T_{, i}}{T} \geq 0
$$

where $\mathrm{A}_{n}, \mathrm{~B}_{m}$ and $\Omega_{p q r s}$ are the thermodynamic conjugates to the ISVs $\alpha_{n}, \beta_{m}$ and $w_{p q r s}$ respectively.

Following Lubliner's (1972) approach the strain can be decomposed into the form

$$
\varepsilon_{i j}=\varepsilon_{i j}^{v}\left(\sigma_{i j}, \alpha_{n}, w_{p q r s}, T\right)+\varepsilon_{i j}^{p}\left(\beta_{m}, w_{p q r s}, T\right)
$$

and could be shown that Gibbs's free energy from Equation (4.22) could be also decomposed into the form

$$
\phi=\phi^{v}\left(\sigma_{i j}, \alpha_{n}, w_{p q r s}, T\right)+\phi^{p}\left(\sigma_{i j}, \beta_{m}, w_{p q r s}, T\right)
$$

where $\phi^{v}$ and $\phi^{p}$ are the Gibbs's free energies associated with viscoelastic and permanent deformation respectively. Also it can be shown that this would lead to

$$
\varepsilon_{i j}^{v}=-\frac{\partial \phi^{v}}{\partial \sigma_{i j}}
$$

Equations (4.25) and (4.30) and equations (4.26) and (4.30) would yield

$$
\begin{aligned}
& \mathrm{A}_{n}=-\frac{\partial \phi^{v}}{\partial \alpha_{n}} \\
& \mathrm{~B}_{m}=-\frac{\partial \phi^{p}}{\partial \beta_{m}}
\end{aligned}
$$

Similarly, Equations (4.27) and (4.30) would yield 


$$
\Omega_{p g r s}=\Omega_{p q r s}^{v}+\Omega_{p q r s}^{p}
$$

where

$$
\begin{gathered}
\Omega_{p q r s}^{v}=-\frac{\partial \phi^{v}}{\partial w_{p q r s}} \\
\Omega_{p q r s}^{p}=-\frac{\partial \phi^{p}}{\partial w_{p q r s}}
\end{gathered}
$$

Finally, dissipation inequality (Equation 4.28) would lead to the following restrictions

$$
\begin{gathered}
\mathrm{A}_{n} \dot{\alpha}_{n} \geq 0 \\
\mathrm{~A}_{n} \dot{\alpha}_{n}+\Omega_{p q r s}^{v} \dot{w}_{p q r s} \geq 0 \\
\mathrm{~B}_{m} \dot{\beta}_{m} \geq 0 \\
\mathrm{~B}_{m} \dot{\beta}_{m}+\Omega_{p q r s}^{p} \dot{w}_{p q r s} \geq 0
\end{gathered}
$$

Inequality (4.36a) should always be satisfied whenever viscoelastic deformation occurs and inequality (4.36b) should always be satisfied whenever viscoelastic deformation is accompanied with damage. Similarly, inequalities (4.37a) and (4.37b) should be satisfied for permanent deformation and permanent deformation accompanied with damage, respectively.

Coupling between permanent deformation and damage has been successfully developed by numerous authors (e.g. Ju, 1989). Instead we will proceed by coupling viscoelastic deformation with damage. We will start with the isothermic case with fixed stress and damage. In this case when the irreversible thermodynamic process is triggered the viscoelastic internal state variables $\alpha_{n}$ will be prompted to drift to their equilibrium values $\alpha_{n}^{e}$. Hence

$$
\alpha_{n}^{e}=\alpha_{n}^{e}\left(\sigma_{i j}, w_{p q r s}\right)
$$

These equilibrium values are assumed to be continuum and differentiable functions of their arguments. Based on this necessary and sufficient condition we can expand the dissipation energy associated with viscoelastic deformation into the Taylor series around its equilibrium value at $\alpha_{n}^{e}$ 


$$
\phi^{v}=\phi_{e}^{v}+\frac{1}{1 !}\left(\frac{\partial \phi^{v}}{\partial \alpha_{n}}\right)_{e}\left(\alpha_{n}-\alpha_{n}^{e}\right)+\frac{1}{2 !}\left(\frac{\partial^{2} \phi^{v}}{\partial \alpha_{n} \partial \alpha_{l}}\right)_{e}\left(\alpha_{n}-\alpha_{n}^{e}\right)\left(\alpha_{l}-\alpha_{l}^{e}\right)+\cdots
$$

Since at equilibrium $\phi^{v}$ is minimum (Callen, 1960), two conditions must be satisfied, i.e.

$$
\begin{gathered}
\left(\frac{\partial \phi^{v}}{\partial \alpha_{n}}\right)_{e}=0 \text { and }\left(\frac{\partial^{2} \phi^{v}}{\partial \alpha_{n} \partial \alpha_{l}}\right)_{e} \delta \alpha_{n} \delta \alpha_{l}>0 \text { therefore Equation (4.38) can be reduced to } \\
\phi^{v}=\phi_{e}^{v}+\frac{1}{2} \phi_{n l}^{v}\left(\alpha_{n}-\alpha_{n}^{e}\right)\left(\alpha_{l}-\alpha_{l}^{e}\right)+\cdots
\end{gathered}
$$

Assuming usual viscous like resistance the thermodynamic conjugate $\mathrm{A}_{n}$ can be expressed (Biot, 1954, 1955;Schapery, 1964, 1969)

$$
\mathrm{A}_{n}=\gamma_{n l} \dot{\alpha}_{l}
$$

where $\gamma_{n l}=\gamma_{n l}(T)$ is a symmetric matrix (Fung, 1965) And in the view of inequality (4.36a) the matrix $\gamma_{n l}$ is positive semi-definite. Schapery $(1964,1969)$ has shown that it could be assumed that all $\gamma_{n l}$ have common dependence on $T$ by

$$
\gamma_{n l}=\gamma_{T}(T) \gamma_{n l}^{0}
$$

where $\gamma_{T}$ is a positive scalar valued function of $T$, and $\gamma_{n l}^{0}$ is a constant symmetric positive semi-definite matrix.

Upon substitution of Equation (4.41) into Equation (4.32) and then substitution into Equation (4.39) would lead into differential equation

$$
\gamma_{n l}^{0} \frac{d \alpha_{l}}{d \xi}+\phi_{n l}^{v} \alpha_{l}=\phi_{n l}^{v} \alpha_{l}^{e}
$$

where $\xi$ is the temperature-transformed time (reduced time) and is defined by $d \xi=d t / \gamma_{T}$, which upon integration would lead to

$$
\xi=\int_{0}^{t} \frac{d t^{\prime}}{\gamma_{T}\left(T\left(t^{\prime}\right)\right)}
$$

Physically, the scalar $\gamma_{T}$ represents the time temperature shift factor characteristic of thermorheologically simple materials (Ferry, 1980).

The Equation (4.42) can be rewritten in the diagonal form

$$
\Gamma_{n}^{0} \frac{d \hat{\alpha}_{n}}{d \xi}+\Phi_{n}^{\vee} \hat{\alpha}_{n}=\Phi_{n}^{v} \hat{\alpha}_{n}^{e} \quad \text { (no sum over } \mathrm{n} \text { ) }
$$


The solution of this equation is given by

$$
\hat{\alpha}_{n}=\hat{\alpha}_{n}^{e}\left(1-e^{-\xi / \tau_{n}}\right) \quad \text { (no sum over } \mathrm{n} \text { ) }
$$

where $\hat{\alpha}_{n}$ is a transformed set of ISVs, $\Gamma_{n}^{0}$ and $\Phi_{n}^{e}$ are constants and $\tau_{n}$ are retardation times given by

$$
\tau_{n}=\frac{\Gamma_{n}^{0}}{\Phi_{n}^{v}} \quad \text { (no sum over } \mathrm{n} \text { ) }
$$

We can now rewrite Equation (4.39) in terms of transformed quantities

$$
\phi^{v}=\phi_{e}^{v}+\frac{1}{2} \sum_{n} \Phi_{n}^{v}\left(\hat{\alpha}_{n}-\hat{\alpha}_{n}^{e}\right)^{2}+\cdots
$$

Substituting Equation (4.47) into (4.31) we will get

$$
\varepsilon_{i j}^{v}=-\frac{\partial \phi_{e}}{\partial \sigma_{i j}}-\frac{1}{2} \sum_{n} \frac{\partial}{\partial \sigma_{i j}}\left(\Phi_{n}^{v}\left(\hat{\alpha}_{n}-\hat{\alpha}_{n}^{e}\right)^{2}\right)
$$

after performing the partial derivatives keeping the $\hat{\alpha}_{n}$ constant and regrouping we will get

$$
\varepsilon_{i j}^{v}=-\frac{\partial \phi_{0}}{\partial \sigma_{i j}}+\sum_{n}\left[1-e^{-\xi / \tau_{n}}\right] \frac{\partial \mathrm{K}_{n}}{\partial \sigma_{i j}}
$$

where

$$
\mathrm{K}_{n}=\frac{1}{2} \Phi_{n}^{v}\left(\alpha_{n}^{e}\right)^{2}
$$

and

$$
\phi_{0}=\phi_{n}^{v}+\sum_{n} \mathrm{~K}_{n}
$$

The first term in Equation (4.48) represents the instantaneous strain and the second one is the transient part of the strain.

It is now possible to recast the above formulation into a format that would allow the introduction of damage effects by means of an effective stress into linear viscoelastic compliance. This could be achieved by adapting stress-based continuum damage mechanics formulation (Simo and Ju, 1987; Ju, 1990; Lemaitre, 1992). Considering an isotropic case and following a common practice of CDM we can write the visoelatsic dissipation energy accompanied with damage in the form 


$$
\phi_{e}^{v}=-\frac{1}{2} J_{i j k l}^{e} C_{k l m n} \sigma_{i j} \sigma_{m n}
$$

where $J_{i j k l}^{e}$ is the undamaged equilibrium compliance tensor and $C_{k l m n}$ is a double symmetric fourth order valued function of the damage $w_{p q r s}$ such that when $w_{p q r s}=0$ it reduces to an identity tensor $I_{k l m n}=\frac{1}{2}\left(\delta_{k m} \delta_{\mathrm{ln}}+\delta_{k n} \delta_{l m}\right)$, where $\delta_{i j}$ is Kronecker delta.

It is reasonable to assume that the internal molecular motions that are described by the ISVs describing the viscoelastic deformation occur at much smaller scale than the deformations associated with ISVs describing damage. Hence, it is reasonable to assume that $\hat{\alpha}_{n}^{e}$ and $\mathrm{K}_{n}$ are affected by damage in a common way and thus could be described as having a common dependence on $w_{\text {pqrs }}$. Therefore, similar to Equation (4.51) we can write

$$
\mathrm{K}_{n}=\frac{1}{2} \Delta J_{i j k l}^{t} C_{k l m n} \sigma_{i j} \sigma_{m n} \quad \forall n
$$

Using the relationships (4.51) and (4.52) the instantaneous $\frac{\partial \phi_{0}}{\partial \sigma_{i j}}$ and transient $\sum_{n}\left[1-e^{-\xi / \tau_{n}}\right] \frac{\partial \mathrm{K}_{n}}{\partial \sigma_{i j}}$ parts of the strain in Equation (4.48) would become

$$
-\frac{\partial \phi_{0}}{\partial \sigma_{i j}}=\left[J_{i j k l}^{e} C_{k l m n}-\sum_{n} \Delta J_{i j k l}^{t} C_{k l m n}\right] \sigma_{m n}
$$

and

$$
\sum_{n}\left[1-e^{-\xi / \tau_{n}}\right] \frac{\partial \mathrm{K}_{n}}{\partial \sigma_{i j}}=\sum_{n}\left[1-e^{-\xi / \tau_{n}}\right] \Delta J_{i j k l}^{n} C_{k l m n} \sigma_{m n}=\Delta J_{i j k l} C_{k l m n} \sigma_{m n}
$$

respectively, where

$$
\Delta J_{i j k l}=\sum_{n} \Delta J_{i j k l}^{n}\left[1-e^{-\xi / \tau_{n}}\right]
$$

We can rewrite the expression for viscoelastic strain (Equation (4.48))

$$
\varepsilon_{i j}^{v}=J_{i j k l}^{0} C_{k l m n} \sigma_{m n}+\Delta J_{i j k l} C_{k l m n} \sigma_{m n}
$$

where

$$
J_{i j k l}^{0}=J_{i j k l}^{e}-\sum_{n} \Delta J_{i j k l}^{n}
$$


or

$$
\varepsilon_{i j}^{v}=J_{i j k l} \widetilde{\sigma}_{k l}
$$

where

$$
J_{i j k l}=J_{i j k l}^{0}+\Delta J_{i j k l}
$$

and

$$
\widetilde{\sigma}_{k l}=C_{k l m n} \sigma_{m n}
$$

For the case of isotropic damage

$$
\begin{aligned}
& w_{k l m n}=w I_{k l m n} \\
& C_{k l m n}=\frac{1}{1-w} I_{k l m n}
\end{aligned}
$$

For time dependent stress and damage the above formulation is equivalent to the viscoelastic response of the material under time dependent effective stress. Hence, the generalization is straight forward, leading

$$
\varepsilon_{i j}^{v}=\int_{0}^{t} J_{i j k l}(t-\tau) \frac{\partial \widetilde{\sigma}_{k l}}{\partial \tau} d \tau
$$

\subsubsection{Damage Evolution Law}

The constitutive formulation developed in the previous section now can be utilized to predict time-dependent response of human cortical bone. Consider onedimensional uniaxial loading condition. In order to complete the formulation it is necessary to propose an evolution law characterizing the damage parameter $w$. We propose that under creep conditions the evolution law can be taken in the form

$$
\dot{w}=\left[\frac{\left\langle\sigma-\sigma_{t h}\right\rangle}{A_{0}(1-w)}\right]^{r}
$$

where $A_{0}$ and $r$ are material constants and $\sigma_{t h}$ is the threshold value of stress. $\langle *\rangle$ denotes the McAuley bracket, meaning that it vanishes if the expression in the bracket is less than zero.

This assumption indicates that below some threshold level of stress the behavior is linear viscoelastic and that the damage starts to accumulate only after the loading exceeds the threshold value of stress. 
Assuming no damage in the virgin material, that is $w=0$ when $t=0$, Equation (4.64) can be integrated

$$
(1-w)^{r} d w=\left[\frac{\left\langle\sigma-\sigma_{t h}\right\rangle}{A_{0}}\right]^{r} d t
$$

resulting in

$$
1-w=\left[1-(r+1)\left[\frac{\left\langle\sigma-\sigma_{t h}\right\rangle}{A_{0}}\right]^{r} t\right]^{1 / r+1}
$$

Theoretically the value of $w$ at the failure is 1. Using this we can get the time of failure after which the specimen should fail. Applying this condition to the Equation above, we will get

$$
t_{F}=\frac{1}{r+1}\left[\frac{A_{0}}{\left\langle\sigma-\sigma_{t h}\right\rangle}\right]^{r}
$$

Hence the damage evolution equation becomes

$$
1-w=\left[1-\frac{t}{t_{F}}\right]^{1 / r+1}
$$

In the view of Equation (4.66), material parameters unique to cortical bone can be readily obtained from long-term creep-rupture tests and subsequent curve fitting of obtained experimental time to failure versus hold stress relationship.

A review of pertinent literature on studies of bone creep behavior suggests that the proposed damage evolution law is an appropriate one for cortical bone. Carter and Caler $(1983,1985,1989)$ in series of papers proposed a cycle-dependent and timedependent cumulative damage model for cortical bone. In order to characterize the failure characteristics of the creep rupture in their tensile fatigue they assumed to the time history of stress magnitude by $\int_{0}^{t} \frac{d t}{t_{F}(\sigma(t))}=1$ (Carter and Caler, 1983). And subsequently they have shown that power relationship is a better fit to their experimental data compared to the exponential one for determination of the function $t_{F}(\sigma(t))$ Hence, they recommended, following Kachanov (1958), the form $t_{F}(\sigma)=A \sigma^{-B}$. In a subsequent 
paper (Carter and Caler, 1985) they supposed the evolutionary equation for damage in the form $\dot{D}=\frac{1}{A \sigma^{-B}}$. This equation is similar to the one proposed in our study except the stress amplitude is replaced with the stress difference beyond some threshold value of stress and also the rate of damage is not constant as it is in their formulation. The constants obtained from those studies were $A=3.02 \times 10^{35} \mathrm{MPa}^{-1} \mathrm{sec}^{-17.95}$ and $B=17.95$. In the later study (Caler and Carter, 1989), they recommended normalization of calculated stresses with the stiffness of the tested specimens to minimize the scatter of the data.

Fondrk and co-workers were the first to utilize steady state creep rate as a damage measure and to demonstrate that bone has a distinct threshold driven behavior. The experimental results from their studies demonstrated that there is an exponential relationship between creep rate and hold stress in the form $\dot{\varepsilon}=\left(\frac{\sigma}{A_{0}^{\prime}}\right)^{1 / A_{1}}$ or $\log (\sigma)=A_{1} \log (\dot{\varepsilon})+A_{0}$ similar to Bailey-Norton Equation (Boyle and Spence, 1983).

Following Carter and Caler and Fondrk, Bowman and co-workers $(1998,1999)$ have performed numerous studies of bone creep failure in order to obtain which component of bone's microstructure is responsible for the observed creep behavior. They also utilized the time-to-failure expression proposed by Fondrk to fit their data and eventually obtain the relationship between time-to-failure and strain-rate.

Based on the studies above we think that the proposed evolution equation in our study is appropriate one and, besides, it includes time dependent changes in damage as it gradually progresses in the material. In addition it includes the time dependent changes of the effective stress for future application in the proposed formulation. 


\subsubsection{Viscoelastic Compliance Tensor}

It was derived in Section 4.3.3. that the viscoelastic compliance tensor $J_{i j k l}$ is given in terms of instantaneous (time-independent) compliance $J_{i j k l}^{0}$ and transient (timedependent) part $\Delta J_{i j k l}$ by

$$
J_{i j k l}(t)=J_{i j k l}^{0}+\Delta J_{i j k l}(t)
$$

where

$$
\Delta J_{i j k l}(t)=\sum_{n} \Delta J_{i j k l}^{n}\left[1-e^{-\xi / \tau_{n}}\right]
$$

For the one-dimensional uniaxial tensile case Equation (4.63) becomes

$$
\varepsilon=\int_{0}^{t} J_{1111}(t-\tau) \frac{d \tilde{\sigma}(\tau)}{d \tau} d \tau=\int_{0}^{t}\left[J_{1111}^{0}+\Delta J_{1111}(t-\tau)\right] \frac{d}{d \tau}\left(\frac{\sigma}{1-w(\tau)}\right) d \tau
$$

Pipkin (1986) had shown that upon replacing the discrete spectrum of retardation times by an integral of continuous spectrum of the right-hand side of Equation (4.69) could be rewritten for one-dimensional case

$$
\Delta J_{1111}=\sum_{n} \Delta J_{1111}^{n}\left[1-e^{-\xi / \tau_{n}}\right]=J_{1} t^{\kappa}
$$

Denoting $J_{1111}^{0}=J_{0}$ and substituting Equation (4.71) and Equation (4.67) into Equation (4.70) we can get the expression for viscoelastic strain in one-dimensional uniaxial case

$$
\varepsilon=\int_{0}^{t}\left[J_{0}+J_{1}(t-\tau)^{\kappa}\right] \frac{d}{d \tau}\left[\sigma\left(1-\frac{\tau}{t_{F}}\right)^{-\frac{1}{r+1}}\right] d \tau
$$

Performing the integration

$$
\varepsilon=J_{0} \sigma \int_{0}^{t} \frac{d}{d \tau} \frac{1}{(1-w)} d \tau+J_{1} \sigma \int_{0}^{t}\left(1-\frac{\tau}{t}\right)^{\kappa} t^{\kappa} \frac{d}{d \tau}\left[1-\frac{\tau}{t} \frac{t}{t_{F}}\right]^{-\frac{1}{r+1}} d \tau
$$




$$
\varepsilon=J_{0} \sigma\left(1-\frac{t}{t_{F}}\right)^{-\rho}+J_{1} \sigma t^{\kappa} \int_{0}^{t}\left(1-\frac{\tau}{t}\right)^{\kappa} \frac{d}{d \tau}\left(1-\frac{\tau}{t} z\right)^{-\rho} d \tau
$$

where $\rho=\frac{1}{r+1}$ and $z=\frac{t}{t_{F}}$. Changing the variable $\tau$ by $u=\frac{\tau}{t}$ which leads that the integration limits are now from 0 to 1 . Finally,

$$
\varepsilon=J_{0} \sigma\left(1-\frac{t}{t_{F}}\right)^{-\rho}+J_{1} \sigma t^{\kappa} F(1, \rho, \kappa+1 ; z)
$$

where $F(1, \rho, \kappa+1 ; z)$ is a hypergeometric function.

The Equation (4.73) allows us to predict the viscoelastic strain generated in a uniaxial tension provided that parameters $J_{0}, J_{1}, r, \kappa, A_{0}$, unique for cortical bone are known.

$t_{F}$ is given by Equation (4.66) and parameters $r$ and $A_{0}$ could be obtained by curve fitting creep-rupture data. Parameters $J_{0}, J_{1}$ and $\kappa$ could be obtained from short term creep tests, loaded below the threshold stress levels (prior to the initiation of damage) and by curve fitting the creep and recovery data by Equations (4.74) and (4.75), respectively (Figure 4.2)

$$
\begin{array}{lrr}
\varepsilon(t)=\left\lfloor J_{0}+J_{1} t^{\kappa}\right\rfloor \sigma & 0<t<t_{0} & \sigma<\sigma_{\text {th }} \\
\varepsilon(t)=J_{1}\left[t^{\kappa}-\left(t-t_{0}\right)^{\kappa}\right\rfloor \sigma & t>t_{0} & \sigma<\sigma_{\text {th }}
\end{array}
$$

Experimentally obtained set of material parameters then could be substituted into Equation (4.73) and the strain response could be predicted. For the stress levels below the threshold Equation (4.73) reduces to Equation (4.74). 


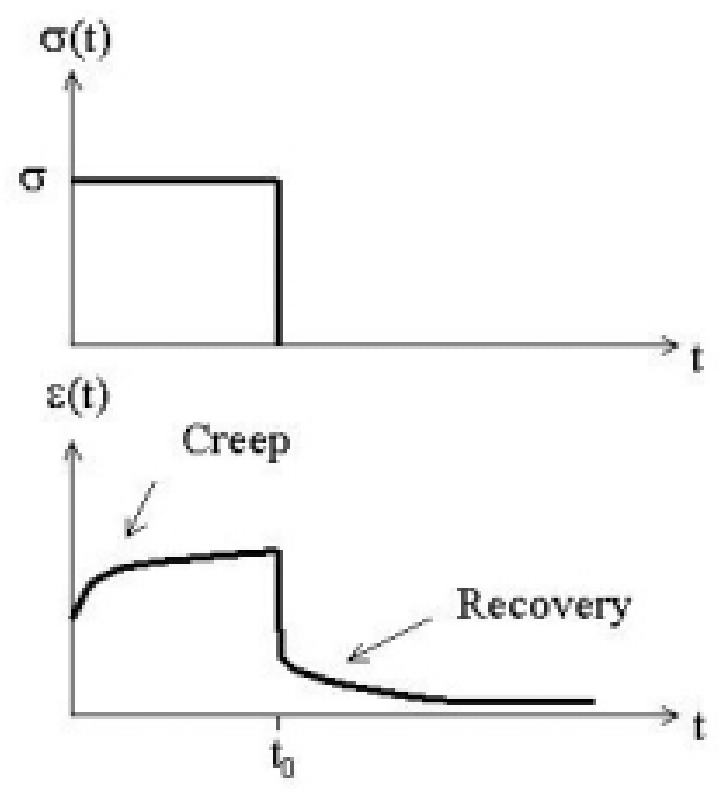

Figure 4.2. Schematic diagram of expected behavior demonstrating typical creep and recovery curves.

\subsection{Objectives}

The main objective of this portion of the study is to utilize the proposed continuum damage model for human cortical bone to predict bone deformation behavior during uniaxial tensile loading. Five sets of experiments would be performed for model development and verification. First, a group of specimens would be tested in order to check the assumptions of thermorheological behavior of the material used in the development of the model. Secondly, a set of specimens would be tested to determine the characteristic threshold value of the stress below which the material is assumed to behave as a linearly viscoelastic material and beyond which material is assumed to behave as a viscoelastic damaging material. This would be achieved by applying loading ramps with 1 minute holding periods and 2 minute recovery periods with successively increasing magnitudes of hold stresses. Thirdly, data from prethreshold loading ramps would be used to obtain the instantaneous (time-independent) and transient (time-dependent) creep compliances of the material. Fourthly, long-term creep-rupture tests would be performed in order to evaluate the proposed damage evolution law and obtain the necessary coefficients by curve fitting the time-to-failure versus hold stress level data. And finally perform 2 step short term creep tests, one ramp below the threshold stress level followed 
by one above the threshold and compare the theoretically predicted deformation with the experimental behavior.

\subsection{Materials and Methods}

\subsubsection{Specimen Preparation}

Four sections were cut from the right tibia of 54 year old male cadaver. Sections were subsequently cleaned from the soft tissue and bone marrow and kept at $-20^{\circ} \mathrm{C}$ wrapped in a $0.9 \%$ saline gaze and in airtight plastic containers until machining. Then three sections were fixed on a vice of a $\mathrm{CNC}$ machine and planed. A code was written and run on the computer controlling the $\mathrm{CNC}$ machine to cut the net geometry of specimens from anteromedial, anterolateral and posterior regions of the tibial sections. Specimens had dog-bone shaped geometry with $1.5 \times 3 \times 15 \mathrm{~mm}^{3}$ (thickness $\times$ width $\times$ length) gage volume. The grip area geometry was $1.5 \times 7 \times 10 \mathrm{~mm}^{3}$. The radius of wasted area was $5 \mathrm{~mm}$ (Figure 4.3)

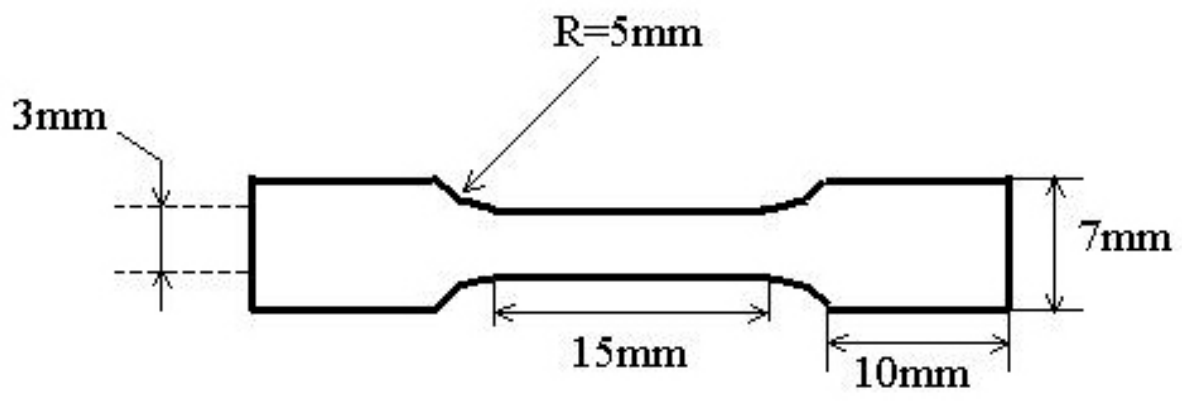

Figure 4.3. Specimen geometry. 
A total of 22 specimens were machined from 3 sections of tibia. The geometry of the specimens and the groups that they have been randomly assigned are summarized in Table 4.1.

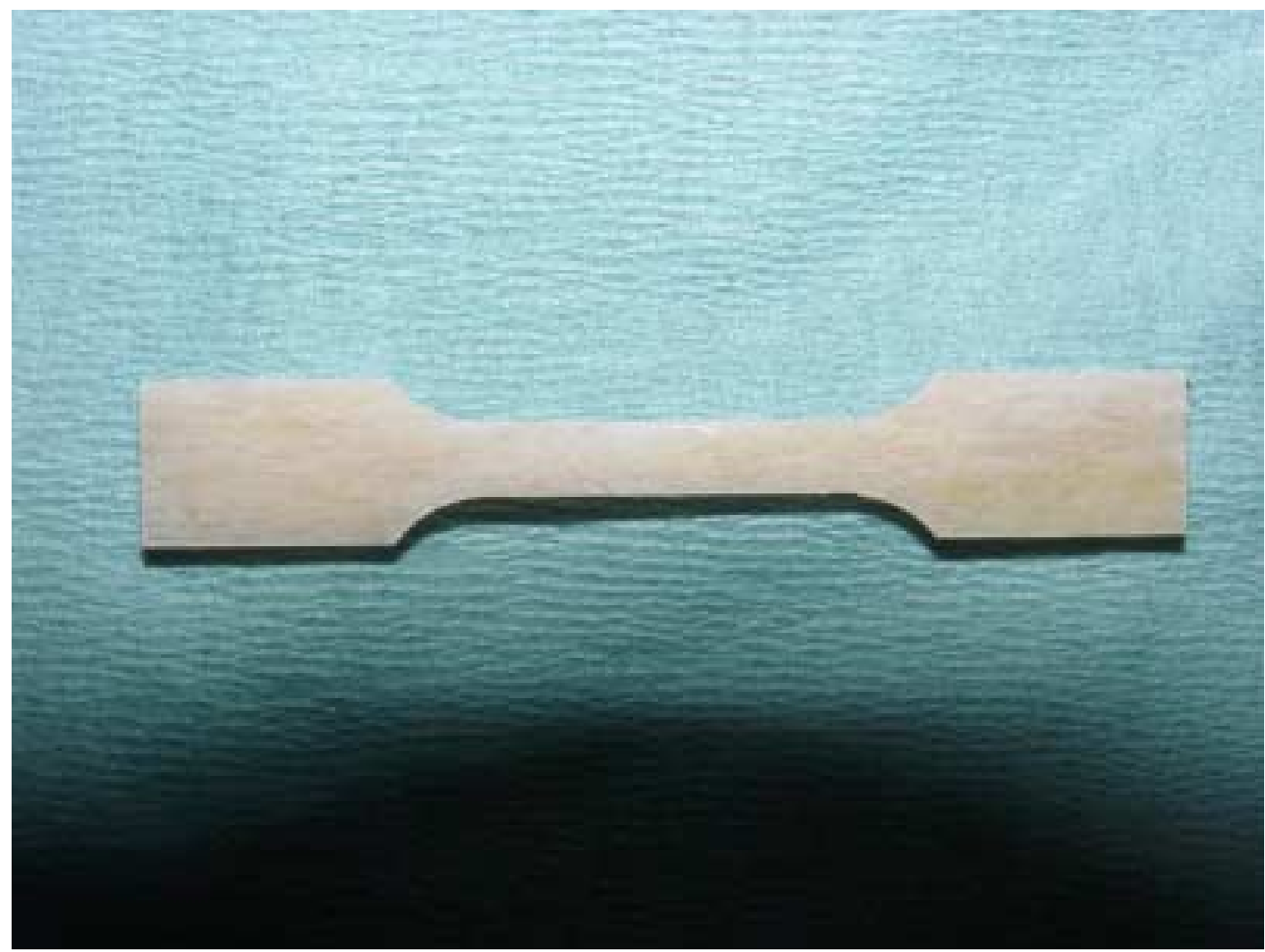

Figure 4.4. A photograph of a bone specimen. 
Table 4.1. Summary of specimens that were tested. R indicates creep rupture test specimens and T-threshold tests specimens.

\begin{tabular}{|c|l|l|l|c|c|}
\hline \multicolumn{5}{|c|}{ Creep-Damage Study Specimens } \\
\hline \multicolumn{7}{|c|}{ Cadaver\# 54M-6111 Tibia } \\
\hline Specimen \# & Test type & Location & Cortex & Thickness $(\mathrm{mm})$ & Area $\left(\mathrm{mm}^{2}\right)$ \\
\hline T1 & threshold & proximal & lateral & 1.35 & 4.05 \\
\hline T2 & threshold & proximal & anterior & 1.3 & 3.9 \\
\hline T3 & threshold(F) & proximal & anterior & $\mathbf{1 . 8}$ & 5.4 \\
\hline T4 & threshold & proximal & medial & 1.65 & 4.95 \\
\hline T5 & threshold & medial & anterior & 1.46 & 4.38 \\
\hline T6 & threshold & distal & posterior & 2.03 & 6.09 \\
\hline T7 & threshold & distal & posterior & 1.46 & 4.38 \\
\hline T8 & threshold & distal & medial & 1.36 & 4.08 \\
\hline T9 & threshold & medial & lateral & 1.37 & 4.11 \\
\hline S2 & short creep & medial & lateral & 2.03 & 6.09 \\
\hline T10 & threshold & medial & anterior & 1.5 & 4.5 \\
\hline R1 & creep rupture & proximal & lateral & 1.4 & 4.2 \\
\hline T11 & creep rupture & proximal & anterior & 1.5 & 4.5 \\
\hline R3 & creep rupture & proximal & anterior & 1.63 & 4.91 \\
\hline R4 & creep rupture & proximal & medial & 1.79 & 5.37 \\
\hline R6 & creep rupture & medial & anterior & 1.45 & 4.36 \\
\hline R7 & creep rupture & medial & anterior & 1.74 & 5.22 \\
\hline R5 & creep rupture & medial & medial & 1.46 & 4.38 \\
\hline R10 & creep rupture(FMTS) & medial & medial & $\mathbf{1 . 5 2}$ & $\mathbf{4 . 5 6}$ \\
\hline R9 & creep rupture & distal & anterior & 1.46 & 4.38 \\
\hline T12 & creep rupture & distal & anterior & 1.74 & 1.74 \\
\hline R8 & creep rupture & distal & medial & 1.28 & 3.83 \\
\hline & & & & & \\
\hline
\end{tabular}




\subsection{Experimental Methods for Determination of Threshold Stress}

A total of 8 specimens were randomly chosen and assigned to group $\mathrm{T}$ for studying damage evolution behavior of cortical bone and for the determination of the threshold value of stress causing damage initiation. A custom made fixture was machined from aluminum with a geometry allowing the wide ends of specimens to be tightly fit. In addition, the width of the fixture in the wasted area was $0.2 \mathrm{~mm}$ larger in order to minimize stress concentration effects on the gage area of specimens. Specimens were placed in the fixture and were tested in tension on the MTS servohydraulic testing system (Figure 4.4). Throughout all tests care was taken to keep the specimens wet at all times.

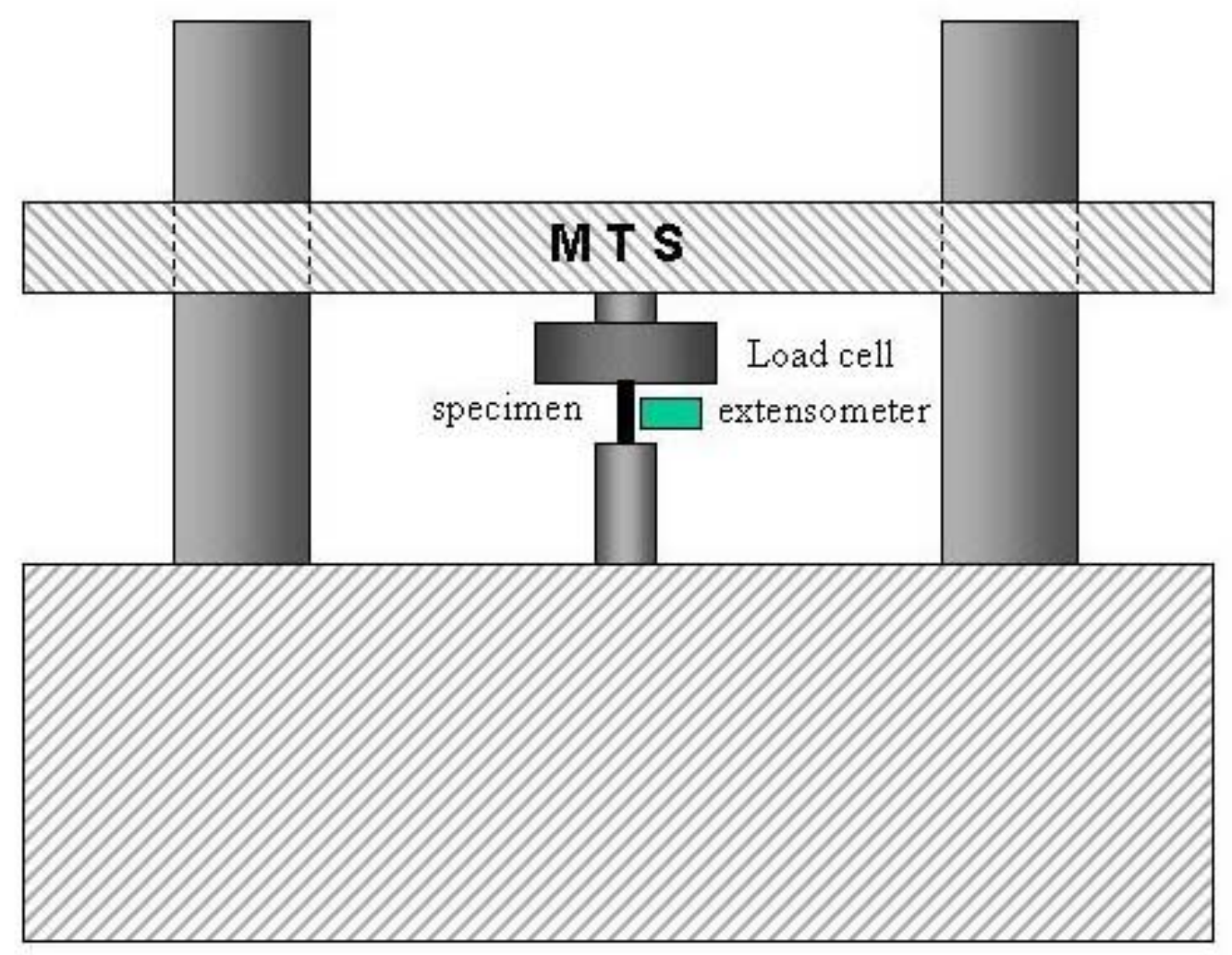

Figure 4.5 Schematic diagram of the experimental setup. 


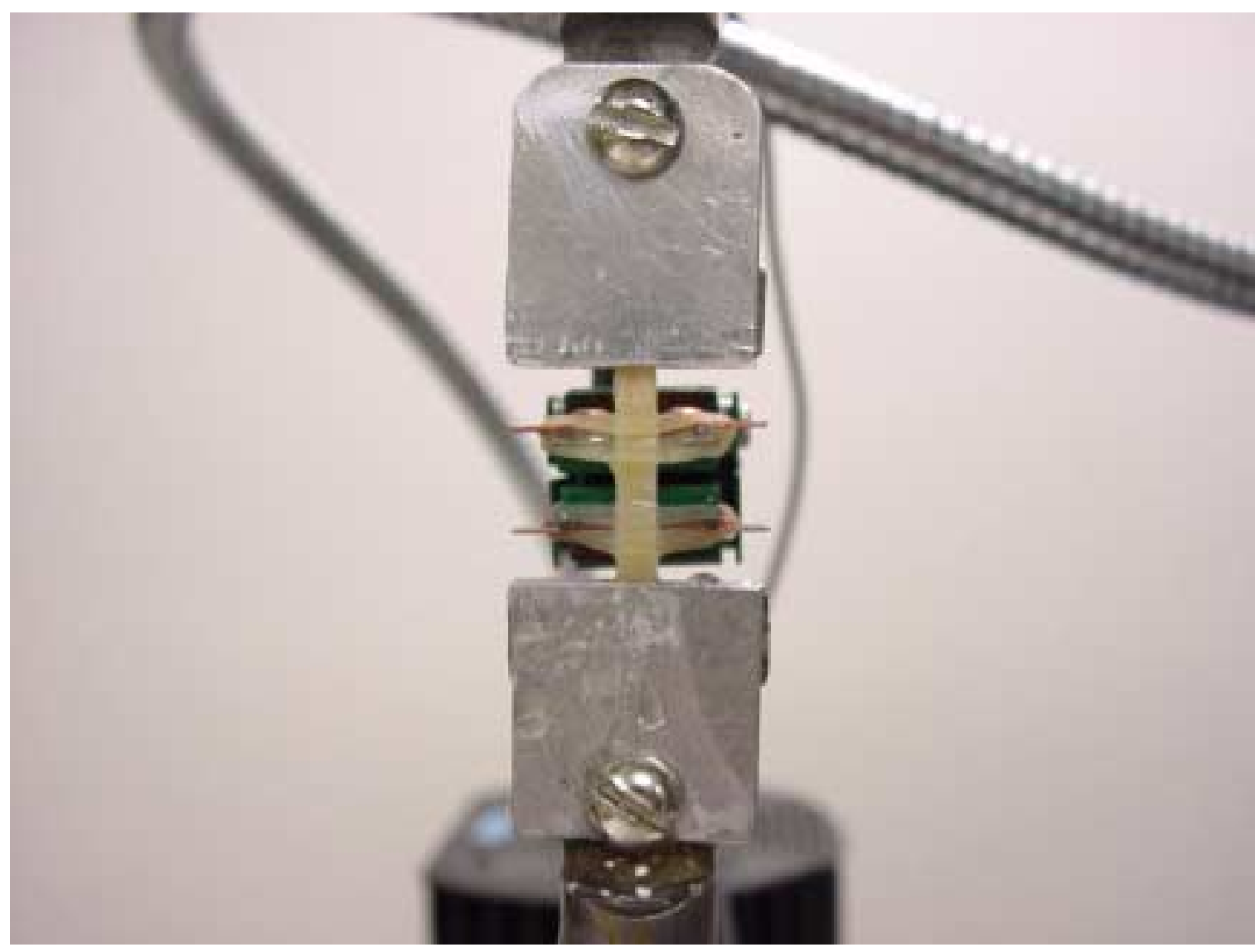

Figure 4.6. A photograph of a specimen in fixture with an attached extensometer.

Loading protocol consisted of ramps with periodically increasing level of hold stress. Each ramp consisted of a loading with a rate of $5 \mathrm{MPasec}^{-1}$ followed by a hold for 60 seconds then unloading at the same rate and rest for 120 seconds. Figure 4.7 demonstrates the typical history of applied loads. Strains were measured by attaching a clip-on extensometer on the gage area of the specimens, which had a gage length of 0.3inches. Data from loading, deformation and the extensometer was collected at a sampling rate of $10 \mathrm{~Hz}$ and stored in the personal computer using LabTech Notebook data acquisition software (Laboratory Technologies Corporation, Wilmington, MA). 

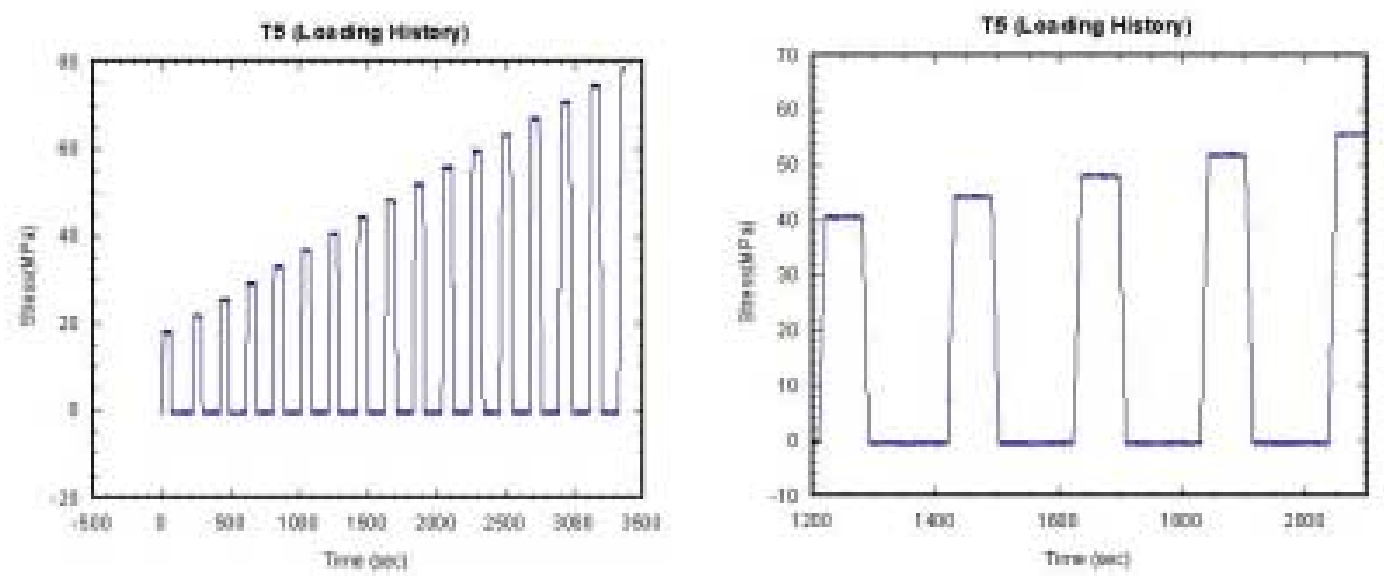

Figure 4.7. Typical loading history for the specimens from the group T. Figure on the right is an expanded view of left graph.

Stresses were subsequently calculated by dividing the applied loads to the cross sectional area of specimens. Prior to tests the extensometer was calibrated in order to obtain the amount of elongation from the data collected in volts. As it was expected, the resulting relationship was linear and is given in Figure 4.8. The obtained relationship was then used to calculate generated strains by dividing the obtained elongations to the length of the gage area of the extensometer (i.e. 0.3 inches).

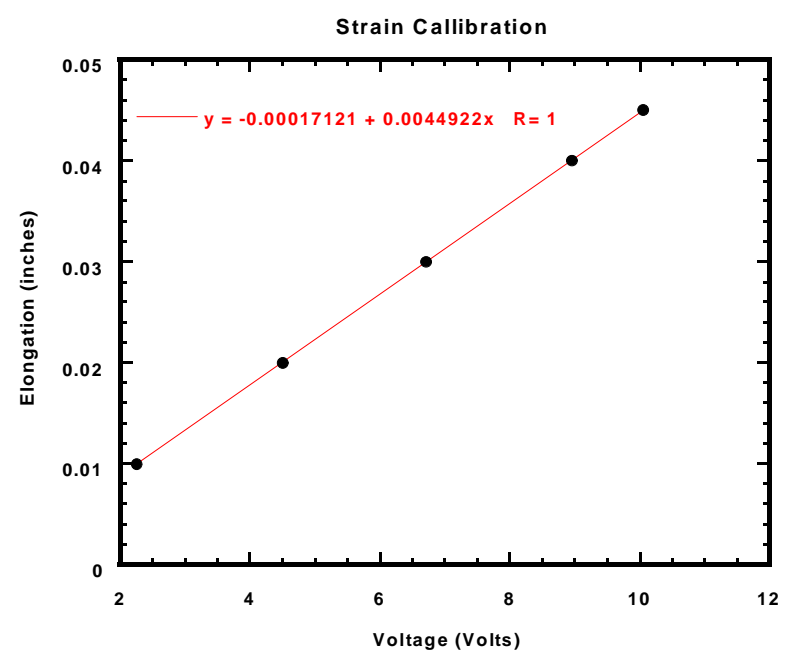

Figure 4.8. This graph represents the relationship between the extensometer reading in volts and the corresponding deflections in inches. 


\subsubsection{Results and Discussion}

There were total of seven $(n=7)$ specimens that were successfully tested for this portion of the study. We will proceed discussing in detail analysis using the data obtained from different specimens in order to avoid repeating the similar discussion on all the specimens individually.

Figures 4.9 and 4.10 demonstrate typical stress-strain curves produced as a result of applying consecutive load cycles. Initial low-stress level cycles failed to induce any damage in the specimens that would have been possible to detect by calculation of the Young's moduli of the successive cycles. Figure 4.9 (B) demonstrates typical behavior observed in all the specimens tested. The Young's moduli obtained from the loading portions of the individual stress-strain curve did not alter as the loading level increased. Another interesting observation that was present in all the specimens tested was that those individual cycles produced hysteretic loops (Figure 4.11). It is known that for the linear elastic materials constant hold stress at the levels below the yield stress should not cause any changes in strain. Therefore after the load is removed the stress-strain curve returns to the origin along the same line.

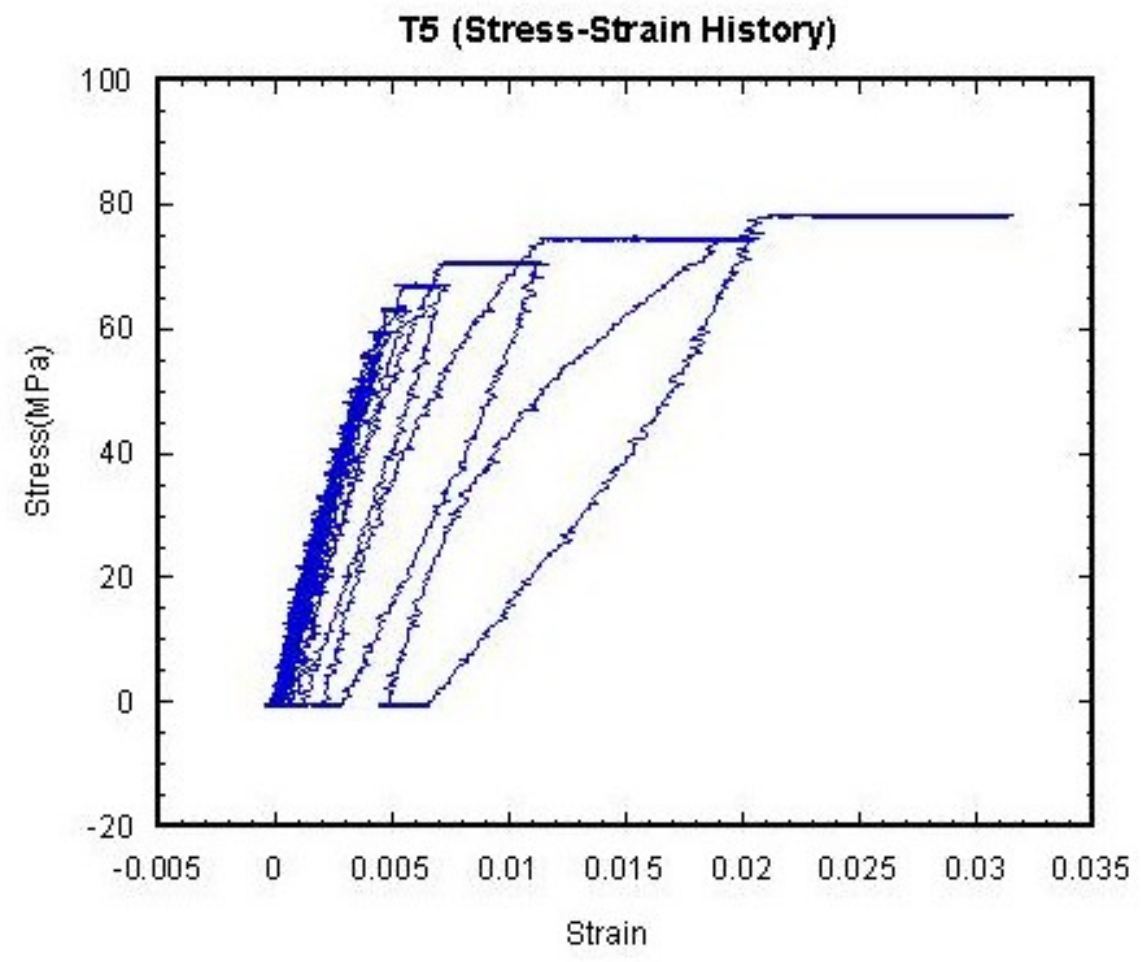




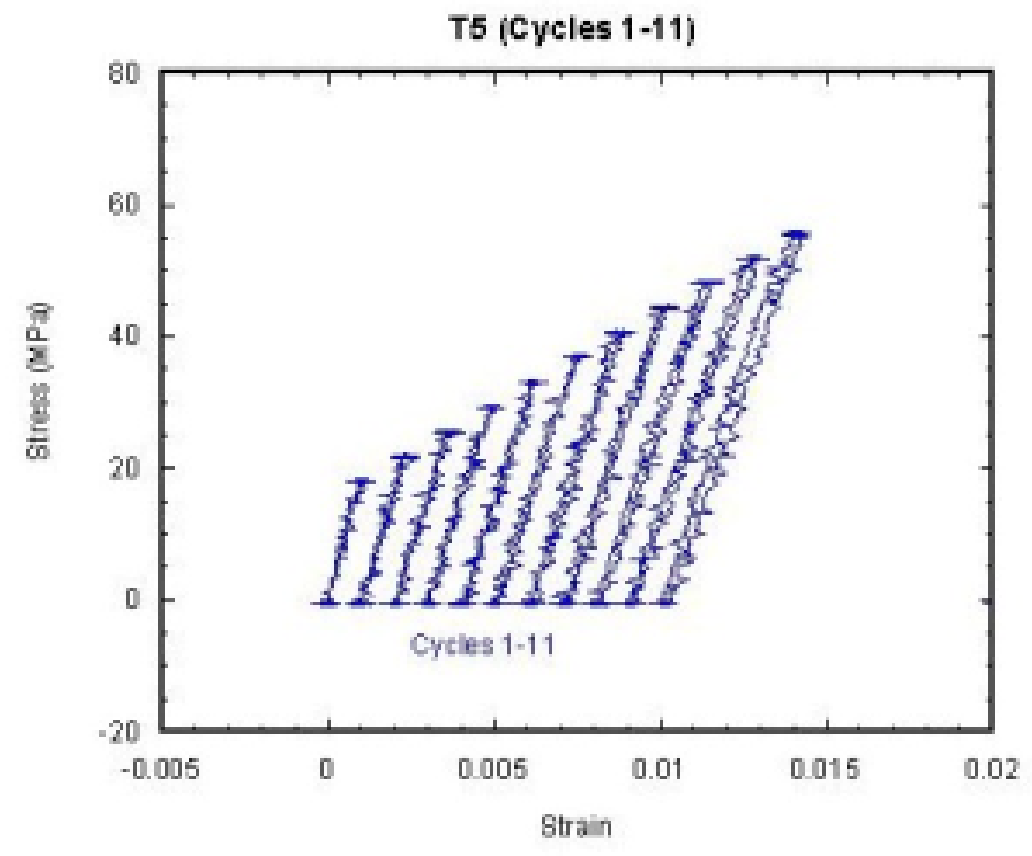

B

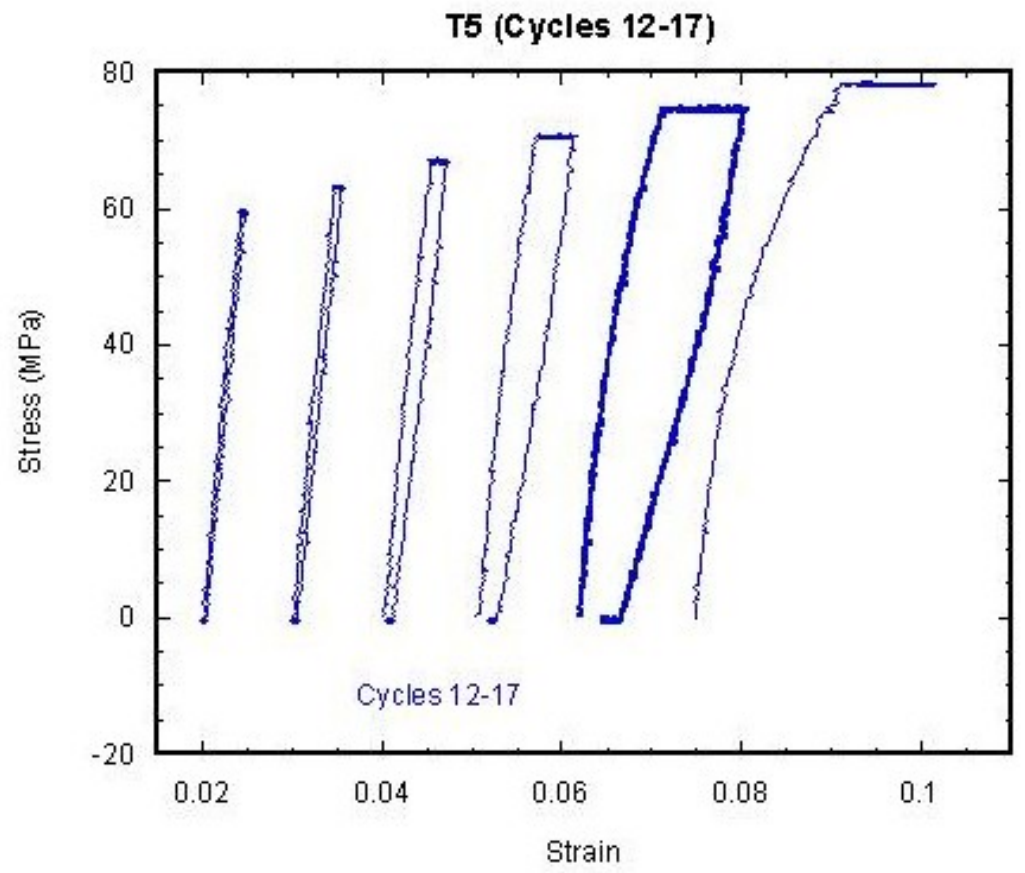

$\mathrm{C}$

Figure 4.9. The stress-strain curves corresponding to the 17 loading ramps (specimen \#T5). The plots B and C are the reproduction of the first plot (A) with shifted strains (1000 and $10000 \mu$ srtains respectively) for the visual clarity purposes. 

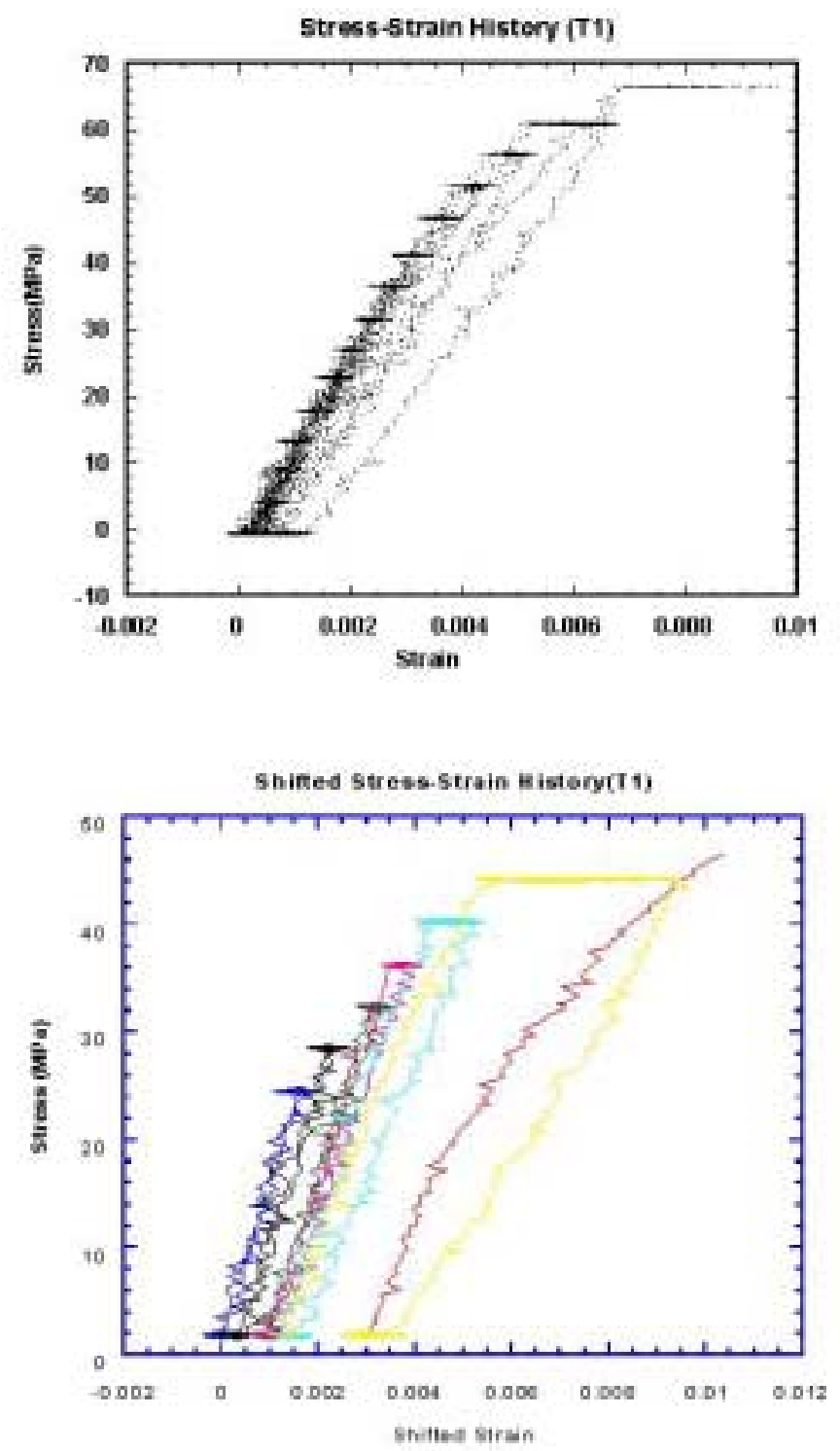

Figure 4.10 Stress-strain curves corresponding to 8 loading cycles (specimen\#T1). The $2^{\text {nd }}$ plot is the reproduction of the first one with the initial strains shifted by $500 \mu$ strains.

In our case there was an evidence of strain increase during the hold stage (Figure 4.11). The typical value of the strain rate during the low hold-stress levels was approximately $2 \mu$ strain/sec. This behavior resembles the one of the linear viscoelastic materials. Hence, the initial assumption about the thermheological nature of bone is supported by our 
experiments. Overall, our results indicate that at low stress levels bone is a linear viscoelastic material.
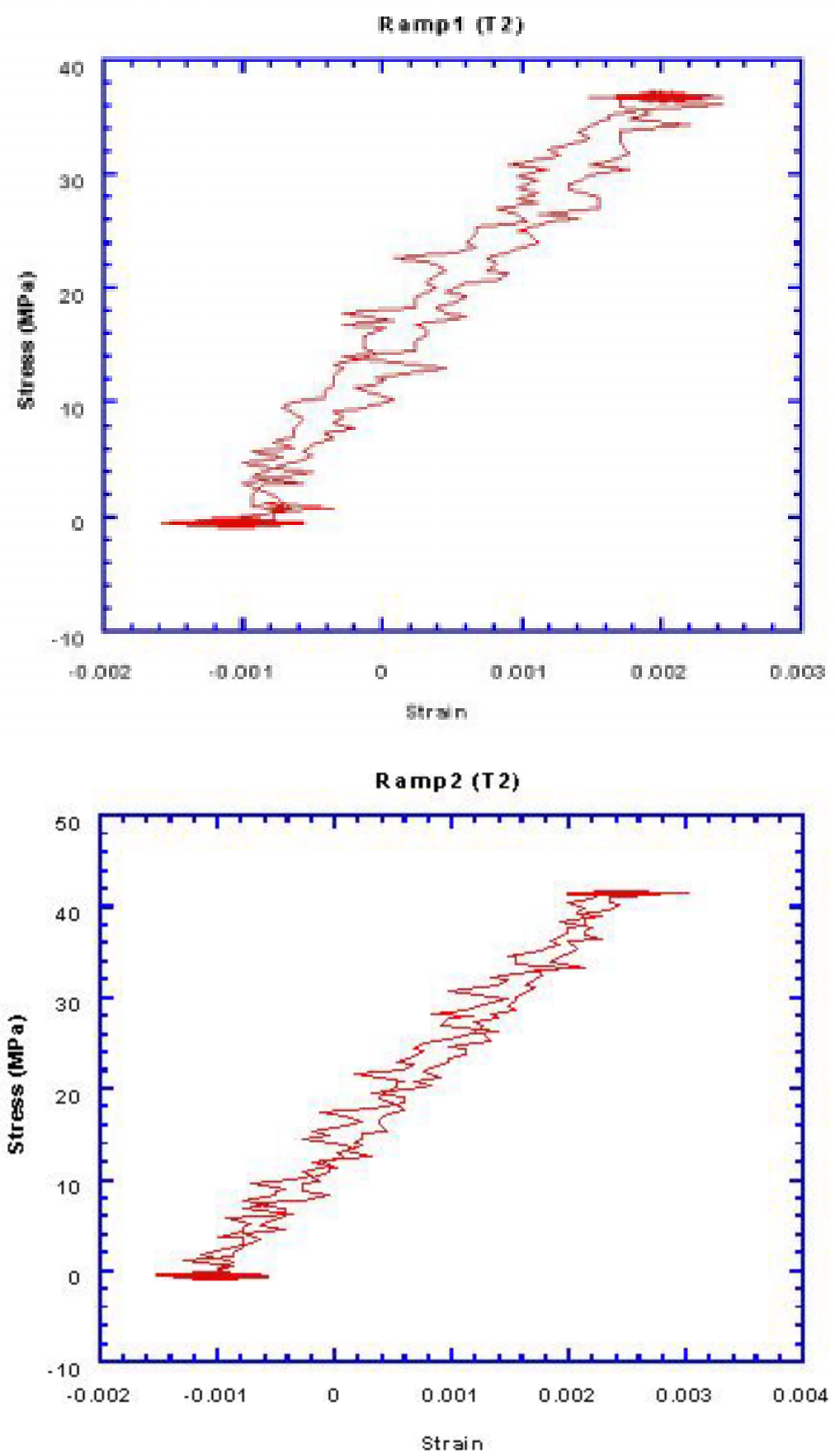

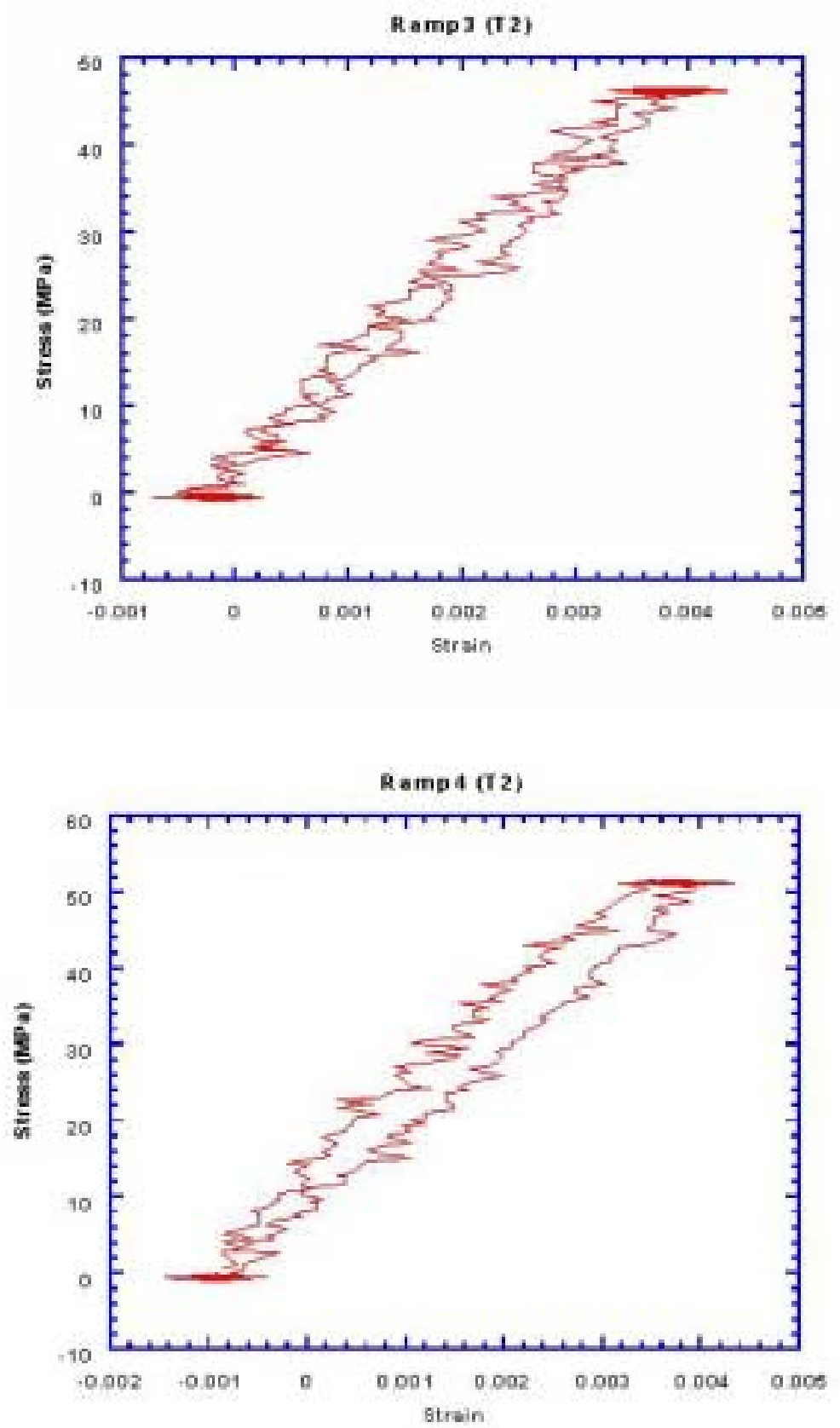


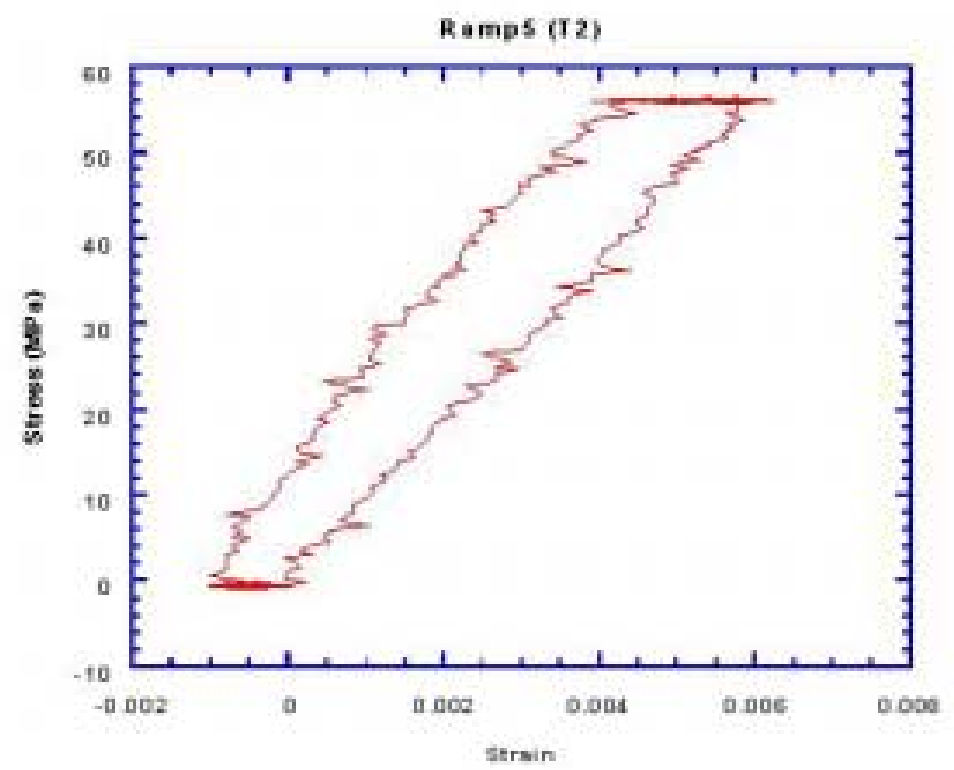

Figure 4.11. Stress-Strain curves of first 5 cycles of the specimen\#T2 demonstrating closed hysteretic loops.

As the load levels increased, the widths of hysteretic loops started to increase, along with appearance of residual strains and the increase in compliance of the specimens (Figures 4.9, 4.10). This behavior could be result of several phenomena, taking place in the specimens. One possible scenario is that as load level increases creep-type mechanisms cause the increased accumulation of strains during the hold stage, which are not recovered after the removal of the load leading to residual strains. But our results show that upon reaching the threshold value of hold stress beyond which the compliance of the specimen starts to increase the hysteretic loops were still closed for at least another two cycles. This behavior is possible if the material is still behaving as a linear viscoelastic material but is accompanied with damage beyond the threshold load level. The stiffness drop is an evidence of damage and hysteretic effect is evidence of vicoelastic behavior.

Figure 4.12 demonstrates history strain as the loading level increases. It can be seen that at the initial stages of loading there is only a small change in strain during the hold stages of loading. As the load level approaches the "threshold" value strain rates during the successive cycles starts to change dramatically. 

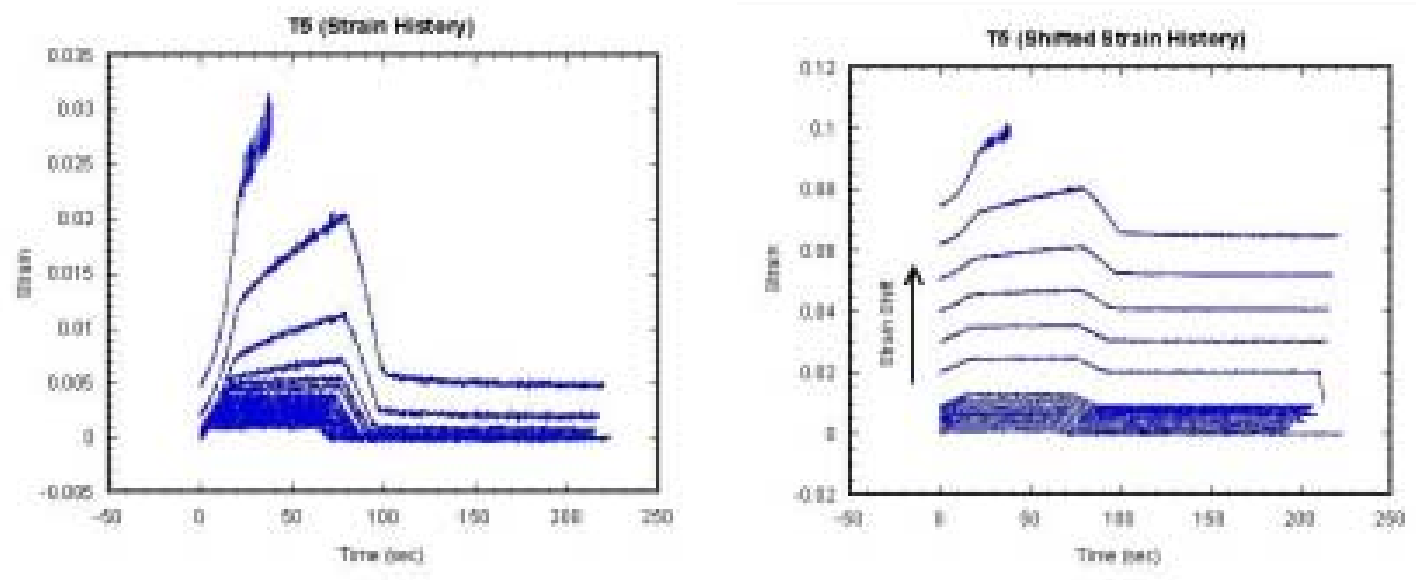

Figure 4.12. Strain vs. time curves produced during the successive loading cycles superimposed on the first loading cycle duration. The 2 nd plot is the reproduction of the $1^{\text {st }}$ one with shifted initial strains for visual clarity.

This is an excellent example of threshold driven nature of damage initiation and accumulation in this material. Hence, this once again supports our choice of the evolutionary equation (Equation (4.64)) for the damage variable, i.e. damage evolution is threshold driven.

In order to verify that the statements made above are accurate we proceeded with the identification of the phenomena, taking place during this transition stage. The data obtained from each of the specimens of group $\mathrm{T}$ was divided into the individual ramps. From each of those ramps the Young's modulus and the strain rate were calculated. For the calculation of the Young's modulus the linear portion of the loading part of the cycle was identified and fit linearly on the stress vs. strain plots (Figure 4.13a). The steadystate creep rate was calculated by fitting the last 40 seconds of the hold part of the cycle (Figure 4.13b). The initial Young's moduli of the specimens is summarized in Table 4.2. 

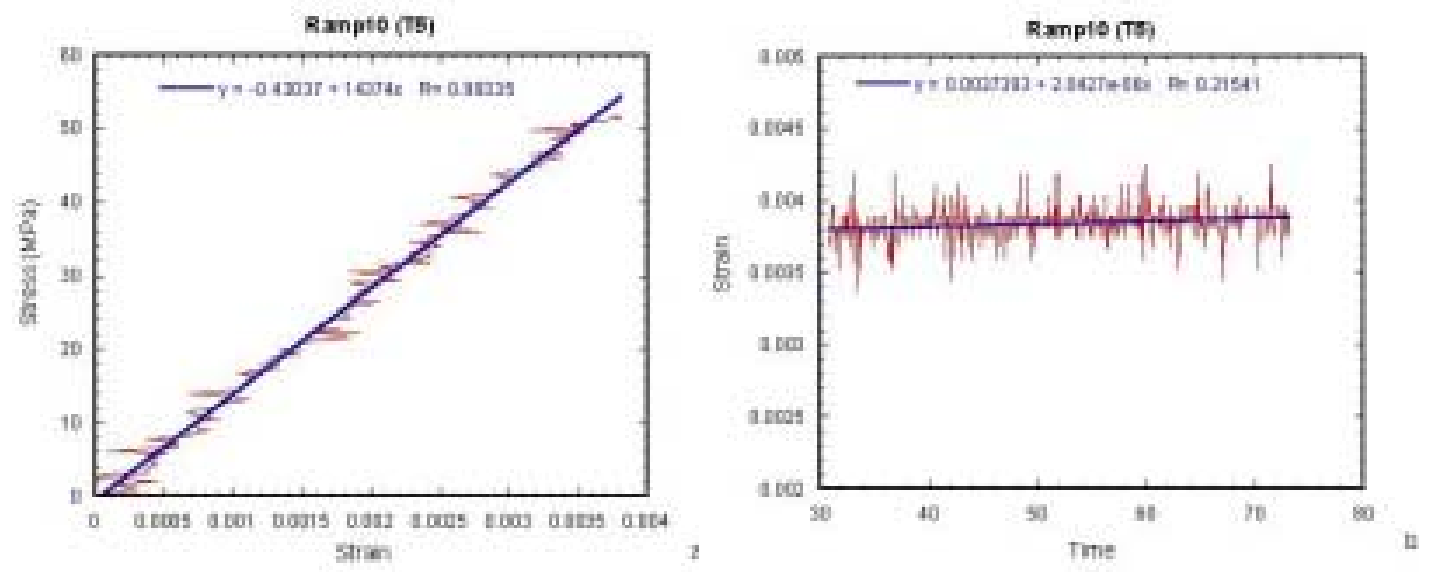

Figure 4.13. (a) Linear portion of the loading part of ramp\#10 of specimen\#T5 is fit linearly to identify Young's modulus (in this case it is E=14.37GPa); (b) The last 40 seconds of the hold part of the same ramp fit linearly to identify strain rate (in this case it is $\dot{\varepsilon}=2.04 \mu$ strain $/ \mathrm{sec}$ ).

Table 4.2. Summary of the initial moduli of the specimens of the group T.

\begin{tabular}{|c|c|}
\hline Specimen\# & Siffness(GPa) \\
\hline T1 & 14.03 \\
\hline T2 & 11.62 \\
\hline T3 & Failed \\
\hline T4 & 14.89 \\
\hline T5 & 15.79 \\
\hline T6 & 16.98 \\
\hline T7 & 17.39 \\
\hline T8 & 15.05 \\
\hline T9 & 18.66 \\
\hline T10 & 20.51 \\
\hline T11 & 20.74 \\
\hline T12 & 22.56 \\
\hline & $\mathbf{1 7 . 1 1} \pm 3.27$ \\
& Mean \pm St.D. $)$ \\
\hline
\end{tabular}


Specimens T1-T8 were tested at the loading rate of $5 \mathrm{MPa} / \mathrm{sec}$. Young's moduli and steady-state creep rates were calculated at each loading cycle. Damage variable $w$ was calculated by dividing the stiffness of each cycle by the initial stiffness, i.e. $w=\frac{E_{N}}{E_{O}}$. where $0 \leq w \leq 1$ and $w=1$ corresponds to no damage and $w=0$ corresponds to idealized failure. Then an expression, defined as (1-w) was plotted against the maximum stress of each cycle (i.e. hold stress). Also, the strain rates obtained from each cycle were plotted against the hold stress. Figure 4.14 demonstrates typical relationships observed. Damage initiation, identified by deviation from 0 in plot $\mathrm{B}$, occurs almost simultaneously as the deviation from an initial constant value of the strain rate, identified by the deviation from a horizontal line in plot A. These observations support our initial assumptions about threshold driven nature of damage initiation. Also, based on the nature of those relationships plotted in Figure 4.14 it could noticed that as the damage remains unchanged at zero, the strain rate is not zero, instead it is a small constant above zero. This is verification supporting our assumption that bone is a linear viscoelastic material. Rapid deviation from the initially constant behavior of both variables occurs simultaneously supports viscoelastic damaging material behavior. This could be readily checked by studying strain recovery behavior beyond the loading and unloading of the threshold value of stress. 


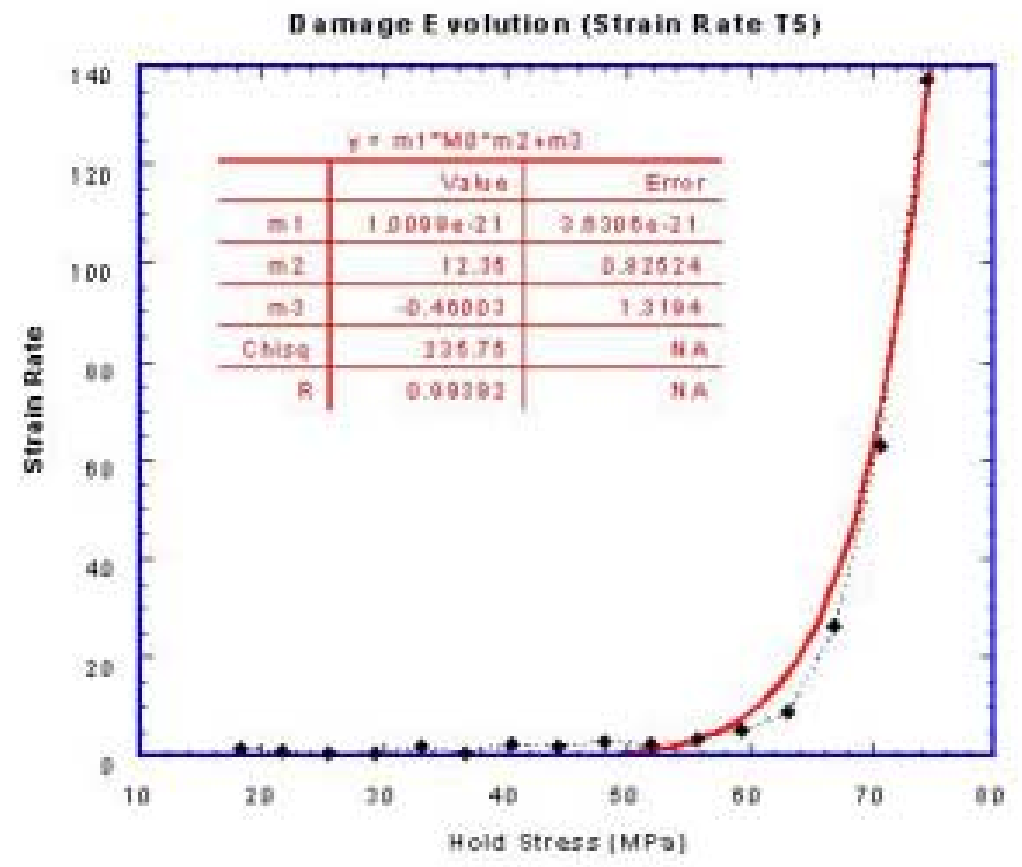

A

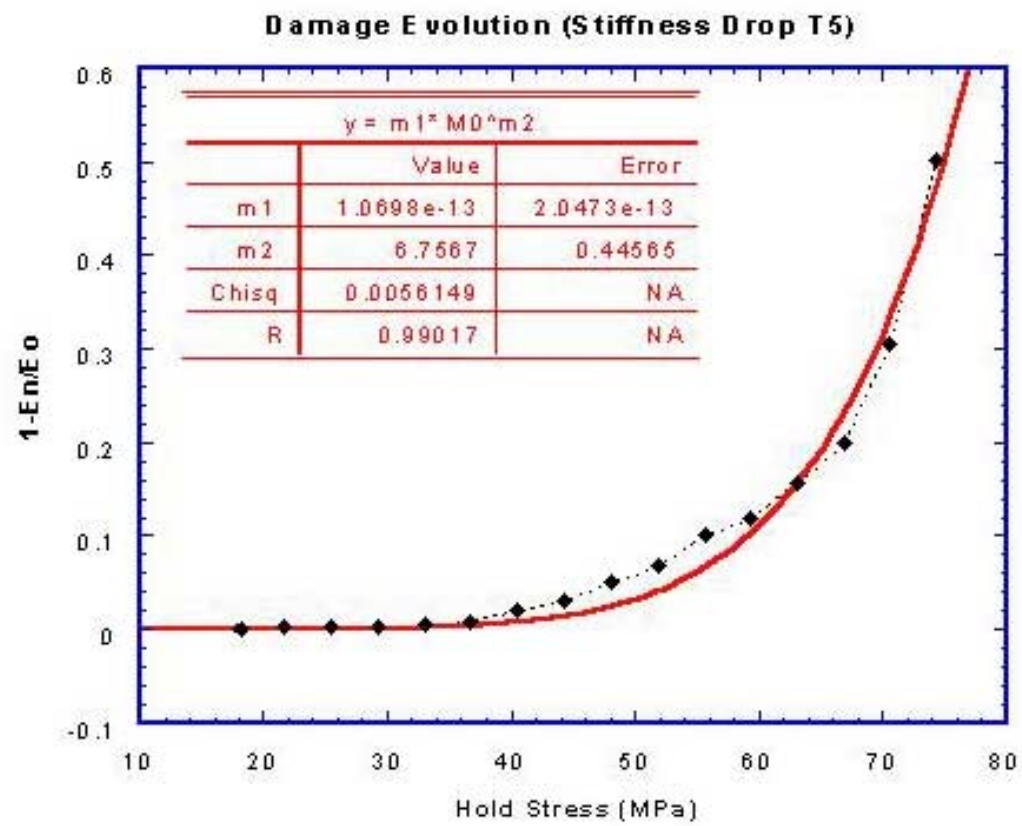

B

Figure 4.14. Typical relationships between the stiffness drop (plot A) and strain rate (plot B) as a function of hold stress. 
If the majority of strain is recovered (70\% per se), this could prove that nonlinear strains accumulated above the threshold are associated with damage as the recovered part of strains would be the viscoelastic strains and permanent part would be the residual strains caused by incomplete reclosure of microcracks generated during damaging processes.

Figures 4.15 and 4.16 demonstrate overall evolutionary relationships of all six $(n=6)$ specimens tested at $5 \mathrm{MPa} / \mathrm{sec}$ stress rate. Figure 4.15 represents the combined plot of all damage evolution curves of specimens T1, T2, T4, T5, T6 and T8.

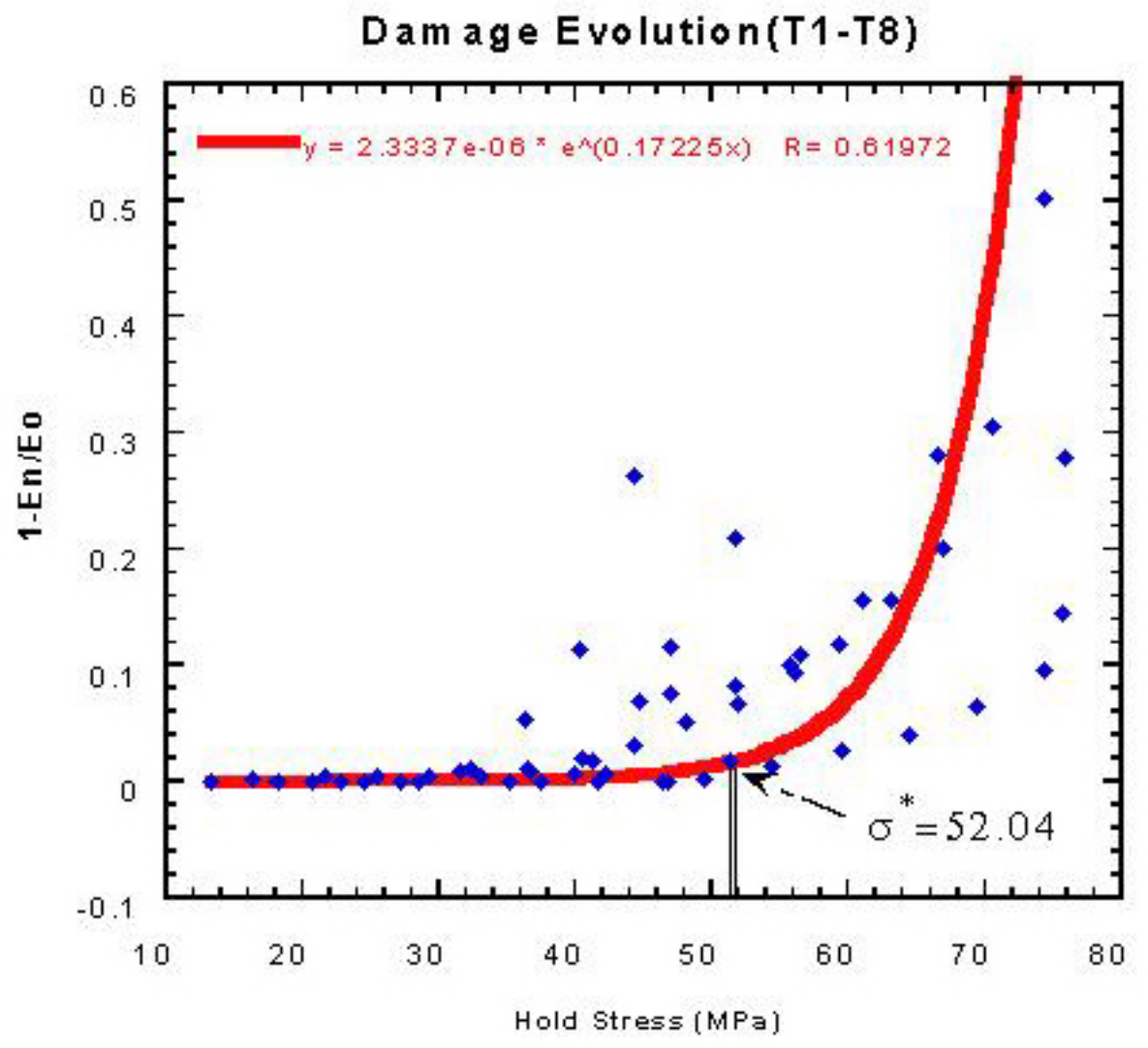

Figure 4.15. The relationship between the stiffness drop and the hold stress for all the specimens from tested at $5 \mathrm{MPa} / \mathrm{sec}$ rate.

The threshold value of stress beyond which damage starts to accumulate was obtained by fitting the data with a function of a type $y=a+\exp (b x)$ and determining the point at which the fitted function deviates from an initial parallel portion. In this case the obtained value of stress was $\sigma_{t h}=52.04 \mathrm{MPa}$. From the individual plots the threshold value ranged from $35 \mathrm{MPa}$ to $54.8 \mathrm{MPa}$. Specimens $\mathrm{T} 2$ and $\mathrm{T} 8$ had noticeably poor 
quality. They consisted of a considerable amount of trabecular bone in their gage area. Thus, it is possible that the calculations of stresses were leading to an underestimation of stresses raised in the gage areas of those specimens. This is possible, because the crosssectional area of the specimens used in the calculation of stresses does not correspond to the accurate actual areas that withstand the loading due to the highly porous nature of the trabecular bone. The threshold values of the rest of the specimens were within 10MPa of the average value.

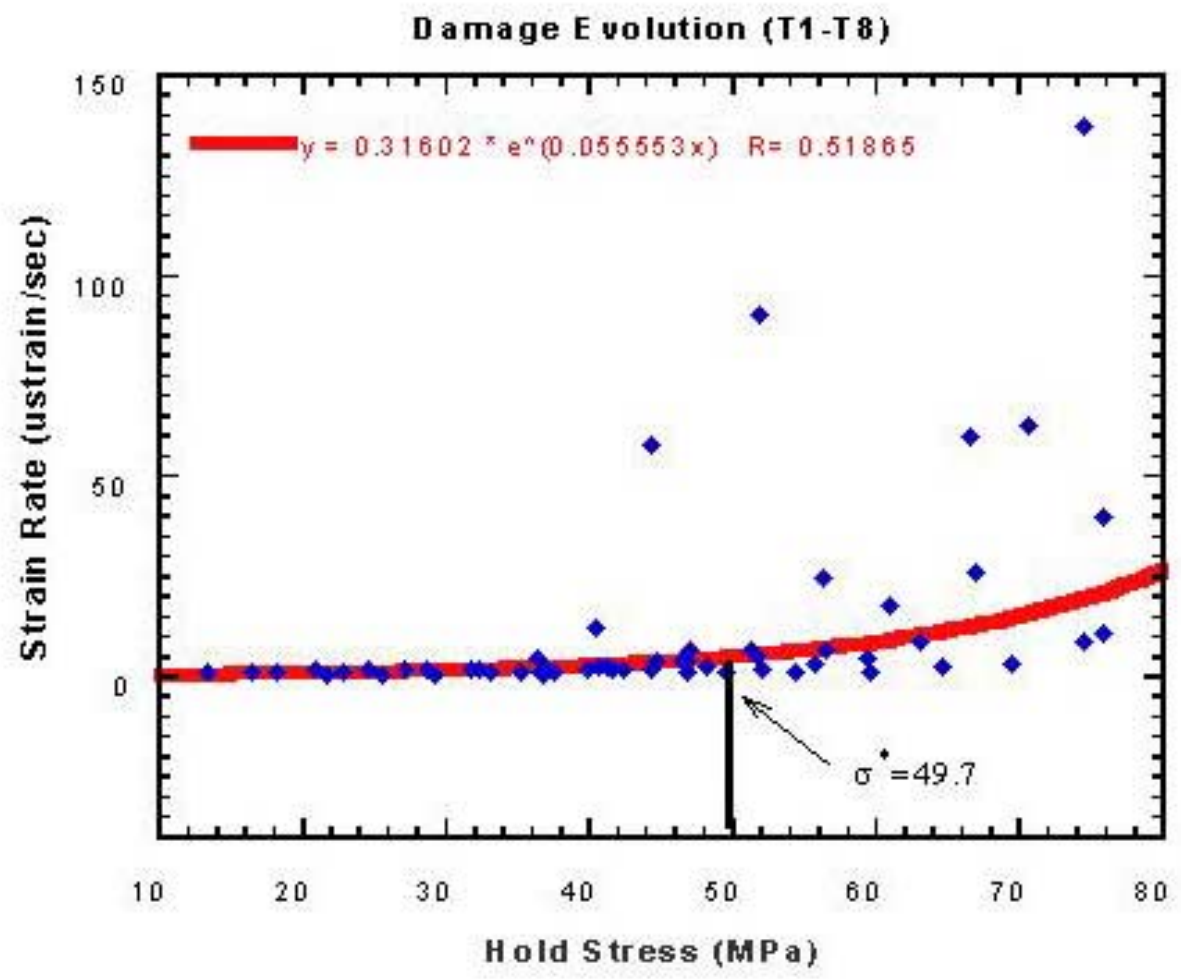

Figure 4.16. The relationship between the strain rate and the hold stress of all the specimens tested at $5 \mathrm{MPa} / \mathrm{sec}$.

In the similar fashion the threshold value of hold stress was obtained from the strain rate data. In this case estimated threshold stress was $49.7 \mathrm{MPa}$. This value is very close to the one obtained earlier $(52.04 \mathrm{MPa})$, thus, once again supporting our arguments mentioned earlier. In addition, it allows us to choose one of these methods for obtaining threshold values of hold stress beyond which damage starts to accumulate. 
For the purpose of obtaining the threshold value of stress that would be applicable to the model proposed in the previous sections the rest of the specimens from the group $\mathrm{T}$ were tested at a very high load rates. This was necessary to avoid any possible viscoelastic dissipation processes that could occur during a slow loading. Also, it was performed in order to avoid performing convolution integration in the verification portion of this study for calculation of strains generated during the loading and hence resemble step loading.

Specimens T7, T9, T10, T11 and T12 were tested at the rate of $80 \mathrm{MPa} / \mathrm{sec}$. This was achieved by adjusting the loading duration of the function generator of the MTS machine. The results in terms of phenomena taking place were similar to the ones discussed earlier. The only differences between the tests performed on this set of specimens and the ones discussed earlier were the loading rate and the increments of loads in each successive loading ramp. We will proceed with determination of the threshold stress for this set of specimens. The relationship between the strain rate and the hold stress was used to estimate the threshold stress level. The result is plotted in Figure 4.17. Various nonlinear functions were utilized to best curve fit the overall data. The best result appeared to be a fit with a function of a type $y=A x^{B}$ where A and B were the parameters to be found. The resulting equation was

$$
\dot{\varepsilon}=2.37 \cdot 10^{-17} \sigma_{H}^{9.07}
$$

Since there are no specific procedures defined for the determination of the onset point of the curve when it starts to deviate from the initial "relatively" constant behavior the following procedure was proposed and utilized. First, the initial linear portion of the data was separated and the fit of the mean was performed using JMP statistical Analysis software (Figure 4.18). As a result the mean and the standard deviation were found to be 2.135119 and 0.454945 respectively. Then a line with an equation of $y=2.135119$ was plotted on the graph with the nonlinear curve fit and was shifted up by the amount of the standard deviation, resulting in a line with equation $y=2.135119+0.454945=2.590064$. Afterwards, the point where this line intersected with the curve fit was defined as the threshold point. The resulting coordinates of that point were $(2.590064 ; 75.29)$. Hence,

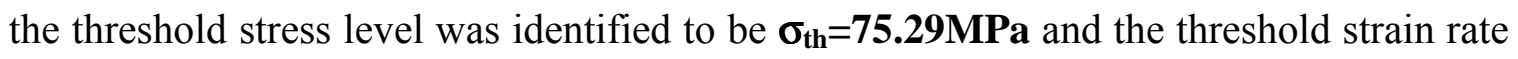


$\dot{\varepsilon}=2.59 \mu$ strain/sec. The threshold value of stress obtained here was then used for the guidance of further experimental analysis.

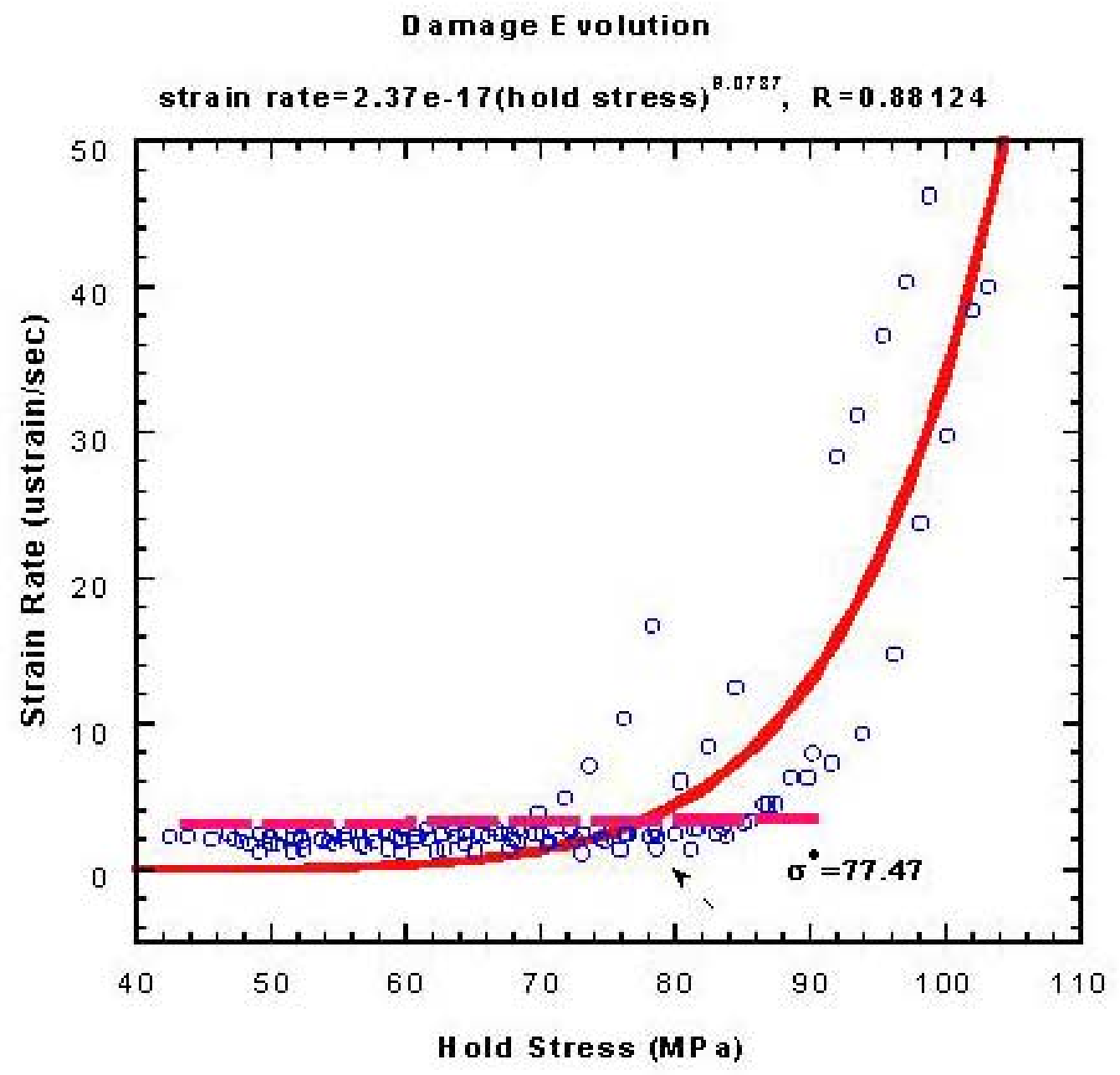

Figure 4.17. The final relationship between the strain rate and the hold stress of all the specimens. 


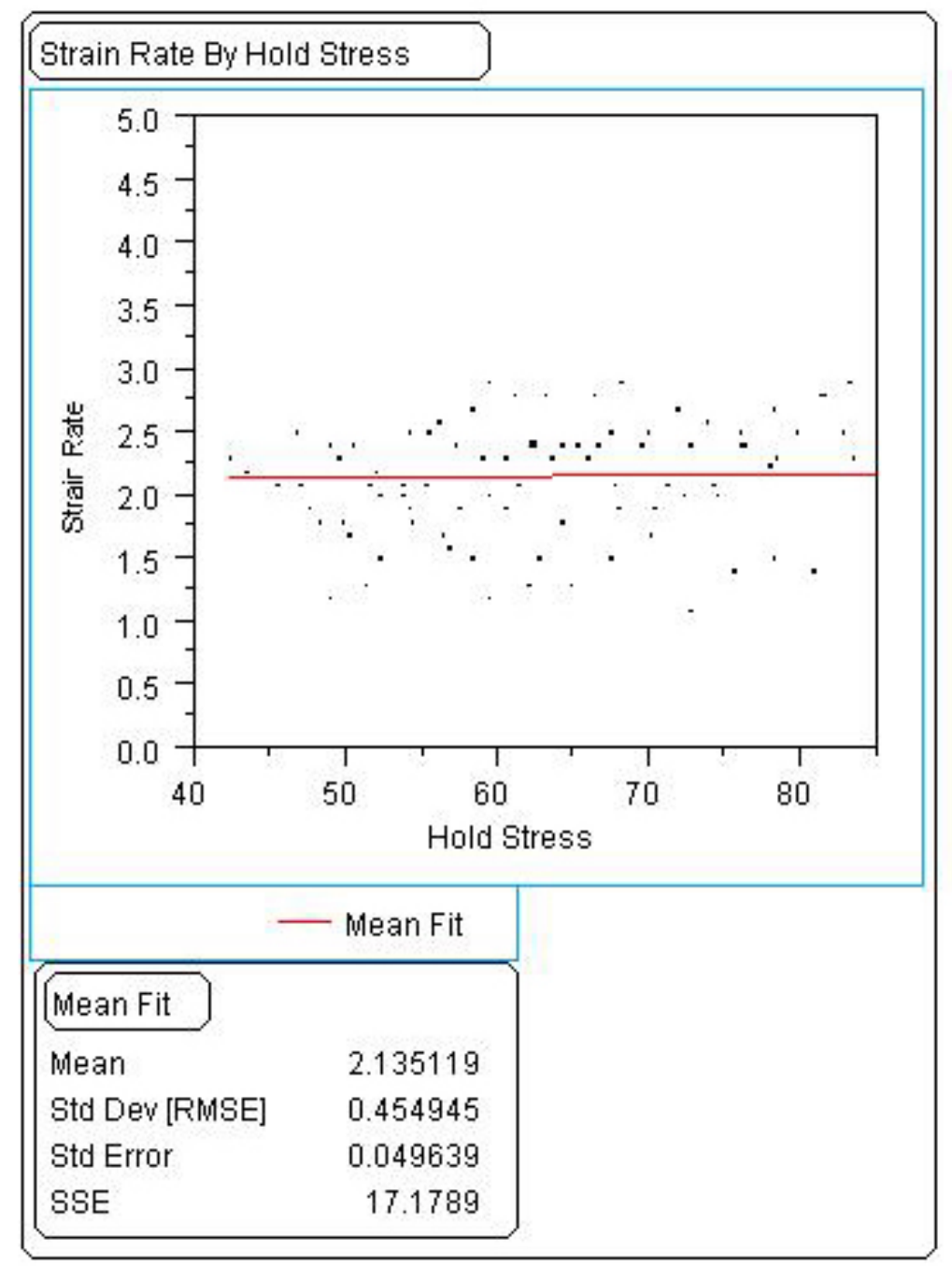

Figure 4.18. The result of the mean fit analysis performed on the initially linear portion of the data from the Figure 4.17.

\subsubsection{Summary}

This portion of the experimental investigation allowed us to demonstrate that key assumptions of the proposed model were correct. First, it was demonstrated that the specimens obtained from the human cortical bone behave as a linearly viscoelastic material. This was concluded based on the evidence of hysteretic loops at low load levels. Secondly, it was shown that damage evolution is threshold driven, meaning that damage remains constant (equal to the initial (in-vivo) amount of damage), then it starts to accumulate nonlinearly after passing some threshold value of stress that the specimen can sustain. It was shown that the onset of damage accumulation temporally coincides with 
onset of the rapid increase in the viscoelasticity-dominated effects. Hence, suggesting that studying damage evolution is equivalent of studying the changes in the viscoelastic response of the material, at least for the determination of the onset of these phenomena. Thirdly, it was determined that creep-type of mechanisms combined with damaging mechanisms are responsible for the behavior beyond the threshold, supporting the approach of the model to separate those two phenomena for more accurate prediction of the material's deformation. Finally the threshold values of stress and strain rate were determined to be $75.29 \mathrm{MPa}$ and $2.59 \mu$ strain/sec, respectively.

\subsection{Experimental Methods for Determination of the Viscoelastic Compliance of the Virgin Material.}

\subsubsection{Methods}

In this portion of the study, data obtained from prethreshold cycles of the specimens T7, T9, T10, T11 and T12 were used to obtain another 3 parameters necessary for the model development. Namely, material parameters $J_{0}, J_{1}$ and $\kappa$ from Equation (4.74). These parameters may be obtained from short-term creep tests below the threshold stress. And, since those specimens have undergone short-term creep tests with periodically increasing levels of hold stresses, the data obtained from the prethreshold region may be used to determine those parameters. These parameters are material constants and subsequently should not depend on stress. In order to simultaneously study the interspecimen variability, five different stress levels were chosen, one for each of those five specimens, and the corresponding data were fit using the Equation (4.74).

\subsubsection{Results}

The resulting plots are given in Figures 4.19-23. Hold stress levels were 63.43MPa, 74.73MPa, 66.97MPa, 61.55MPa and 59.66MPa for the specimens $\mathrm{T} 7$, T9, T10, T11 and T12, respectively. The portion of the strain vs. time curve corresponding to the constant stress was fit using the equation (4.74). The resulting parameters are summarized in Table 4.3 
Table 4.3 Summary of viscoelstic parameters obtained from short-term creep tests.

\begin{tabular}{lllll}
\hline \multicolumn{1}{c}{ Specimen \# } & \multicolumn{1}{c}{$\begin{array}{c}\text { Hold Stress } \\
(\mathrm{MPa})\end{array}$} & $\begin{array}{c}\text { Instantaneous } \\
\text { modulus } \\
J_{0}\left(\times 10^{-6} \mathrm{MPa}^{-1}\right)\end{array}$ & $\begin{array}{c}\text { Constant } \\
J_{l}\left(\times 10^{-6} \mathrm{MPa}^{-}\right. \\
1 / \mathrm{sec}^{\mathrm{\kappa}}\end{array}$ & \multicolumn{1}{c}{ Constant $\kappa$} \\
\hline T7 & 63.43 & 37.734 & 12.482 & 0.0695 \\
T9 & 74.73 & 46.262 & 4.621 & 0.089 \\
T10 & 66.97 & 27.432 & 15.6 & 0.055 \\
T11 & 61.55 & 41.349 & 9.998 & 0.0929 \\
T12 & 59.66 & 36.74 & 10.907 & 0.056 \\
\hline & & $\mathbf{3 7 . 9 0 3 4 \pm 6 . 2 0 9}$ & $\mathbf{1 0 . 7 2 1 6 \pm 3 . 5 9 6 4}$ & $\mathbf{0 . 0 7 2 4 \pm 0 . 0 1 6}$ \\
\hline
\end{tabular}

T 7 (R amp 10 s tress $=63.43 \mathrm{MP}$ a)

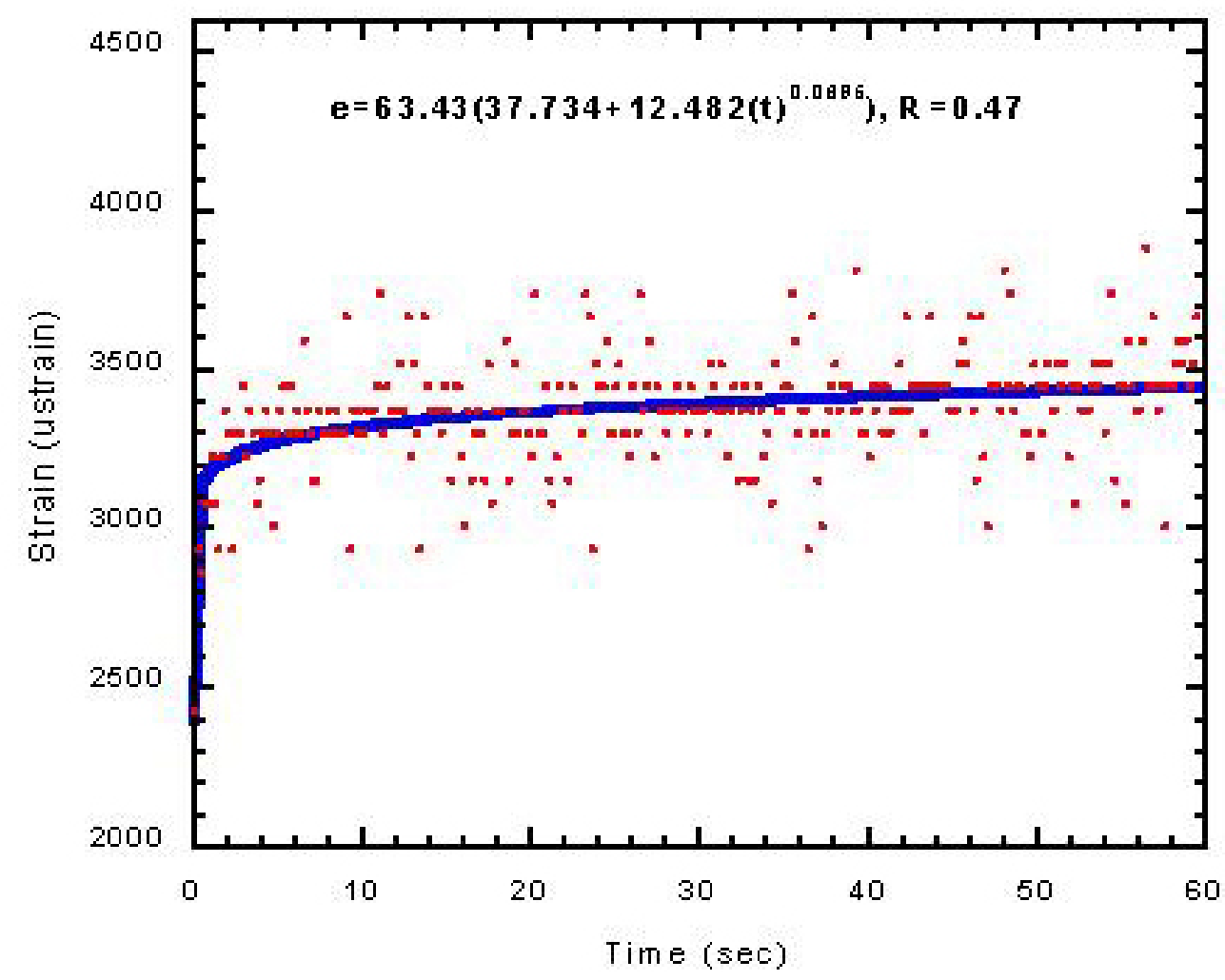

Figure 4.19 Nonlinear fit of the creep strain of the specimen $\mathrm{T} 7$ at a hold stress of 63.43MPa and duration of 60 seconds. 


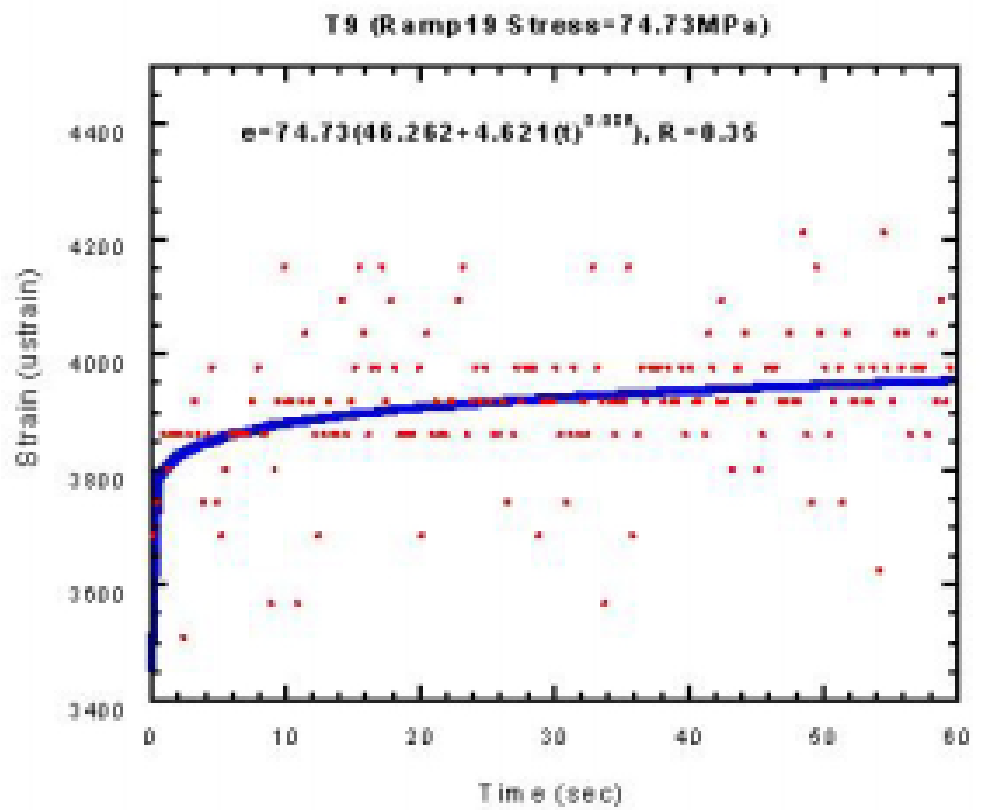

Figure 4.20 Nonlinear fit of the creep strain of the specimen T9 at a hold stress of 74.73MPa and duration of 60 seconds.

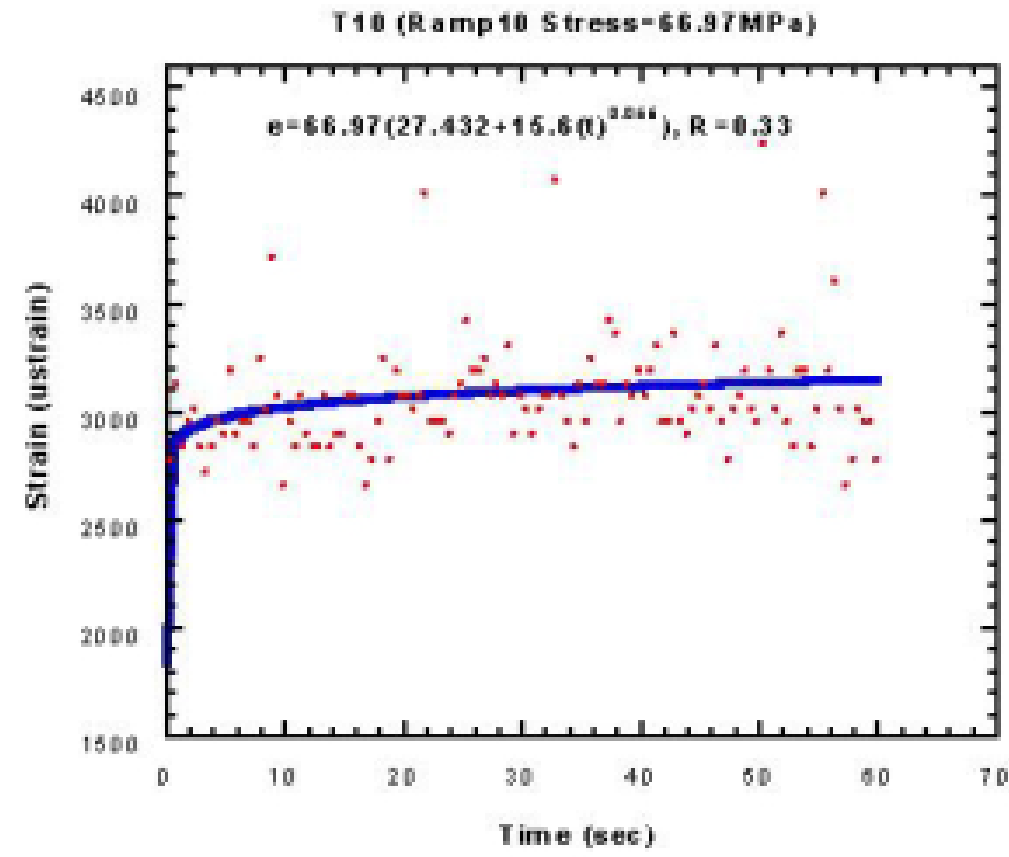

Figure 4.21. Nonlinear fit of the creep strain of the specimen T10 at a hold stress of 66.97MPa and duration of 60 seconds. 


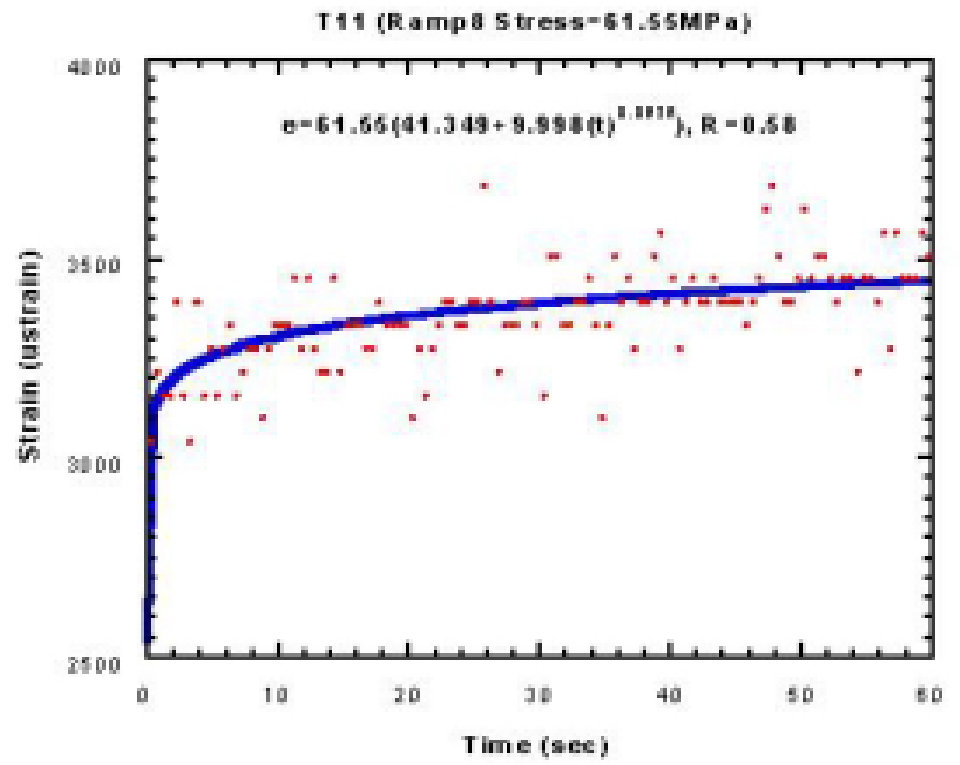

Figure 4.22. Nonlinear fit of the creep strain of the specimen T11 at a hold stress of $61.55 \mathrm{MPa}$ and duration of 60 seconds

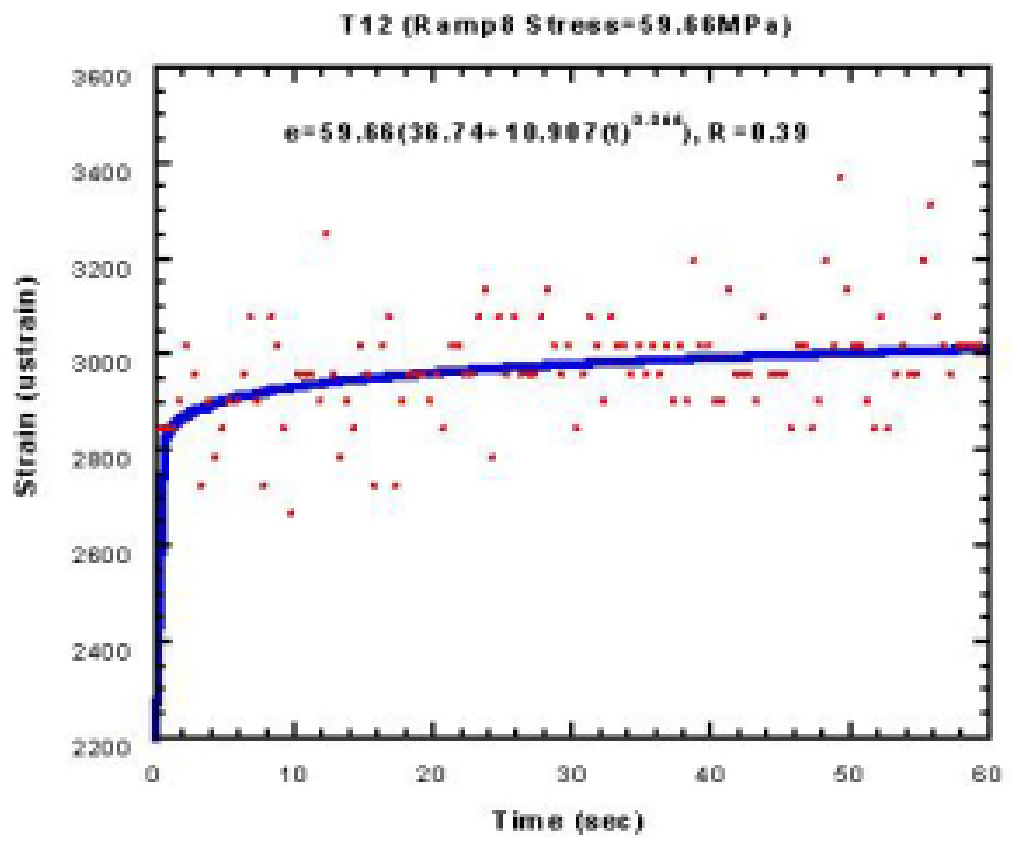

Figure 4.23. Nonlinear fit of the creep strain of the specimen T12 at a hold stress of $59.66 \mathrm{MPa}$ and duration of 60 seconds. 


\subsubsection{Summary}

In this portion of the experimental study we were able to determine the viscoelastic material parameters for the group of the specimens obtained from a human cortical bone. The average value, given in the Table 4.3 would be used for theoretical predictions of the model. Those parameters to be utilized are

$$
\begin{gathered}
J_{0}=37.9034 \times 10^{-6} \mathrm{MPa}^{-1} \\
J_{I}=10.7216 \times 10^{-6} \mathrm{MPa}^{-1}(\mathrm{sec})^{-0.07248} \\
\kappa=0.07248 .
\end{gathered}
$$

\subsection{Experimental Methods for Studying Creep Rupture and Determination of Critical Damage Parameters.}

\subsubsection{Introduction}

In this portion of the study we will proceed to obtain the damage evolution law proposed in the form given by Equation (4.64). It was already shown in section 4.3.4. that this could be achieved by fitting experimental data of creep-rupture using Equation (4.66) and by subsequent substitution into Equation (4.67).

\subsubsection{Methods}

Six ( $\mathrm{n}=6$ ) specimens were assigned to group R (specimens R3, R4, R5, R6, R7, and R8) and were loaded to pre-assigned stress levels above the threshold value of stress $(75.29 \mathrm{MPa})$. determined in the section 4.6. Data acquisition consisted of two stages. During the first stage, with duration of $100 \mathrm{sec}$, data was collected at the sampling rate of $10 \mathrm{~Hz}$. For the second stage the sampling rate was reduced to1Hz. Strains were determined by attaching an extensometer (Epsilon) with a gage length of 0.25 inches along the gage area of specimens. The extensometer was calibrated to convert raw data from the extensometer, given in Volts into the deformation, given in Inches. The resulting equation was

$$
\text { Extension }(\text { Inches })=0.0029967 *(\text { Volts })+0.0000090915 \quad \text { with } \mathrm{R}=0.9999
$$

The above relationship was then used to determine strains by dividing to the gage length 0.25 . 


\subsubsection{Results and Discussion}

Results indicated that bone experiences typical creep behavior observed in other materials. Namely, bone exhibits well defined primary, secondary (steady-state) and tertiary creep behavior. Figures 4.24 and 4.25 demonstrate typical creep curves obtained from the tested specimens.

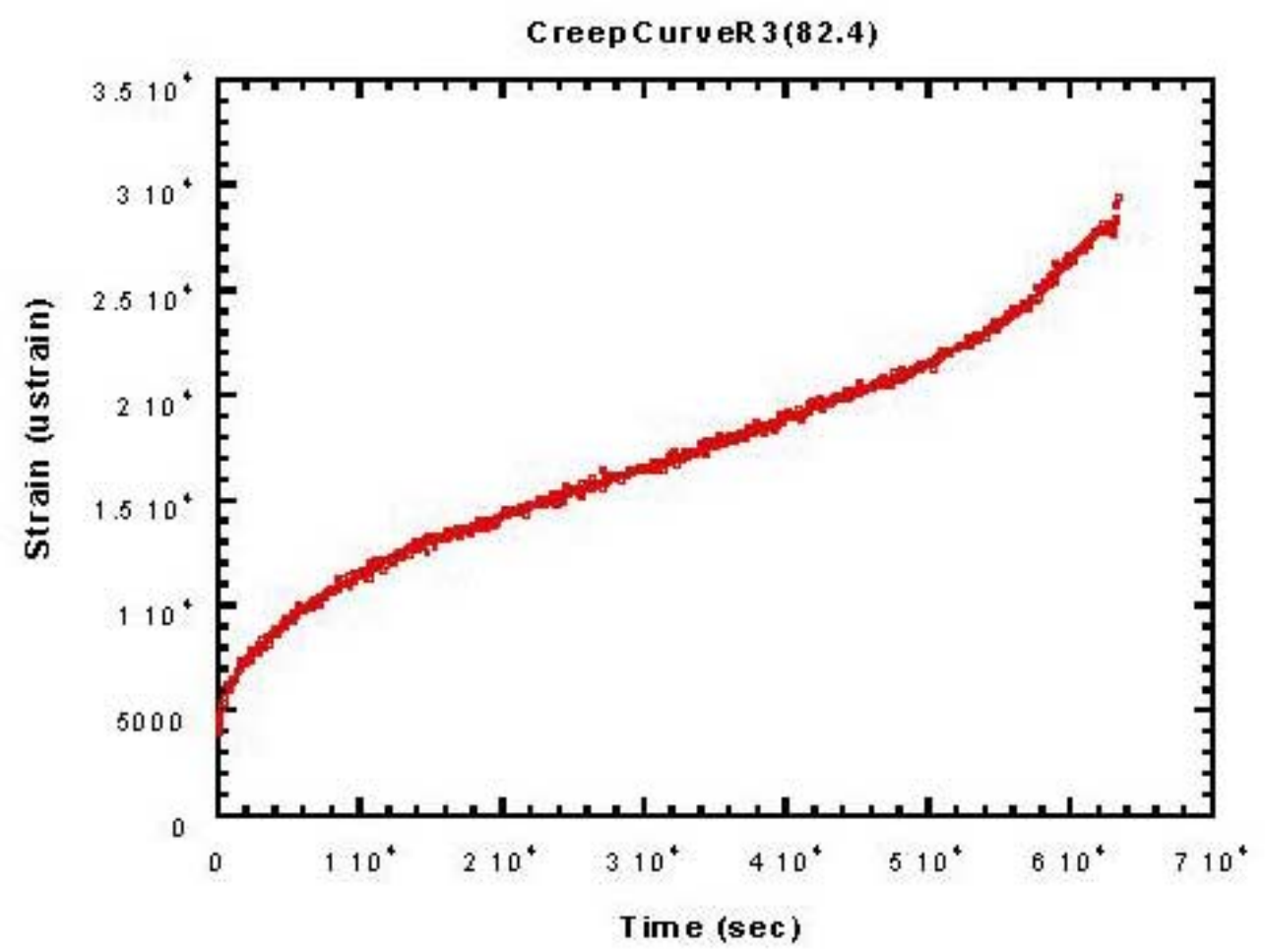

Figure 4.24 Creep curve of specimen R3 held at 82.4MPa. 

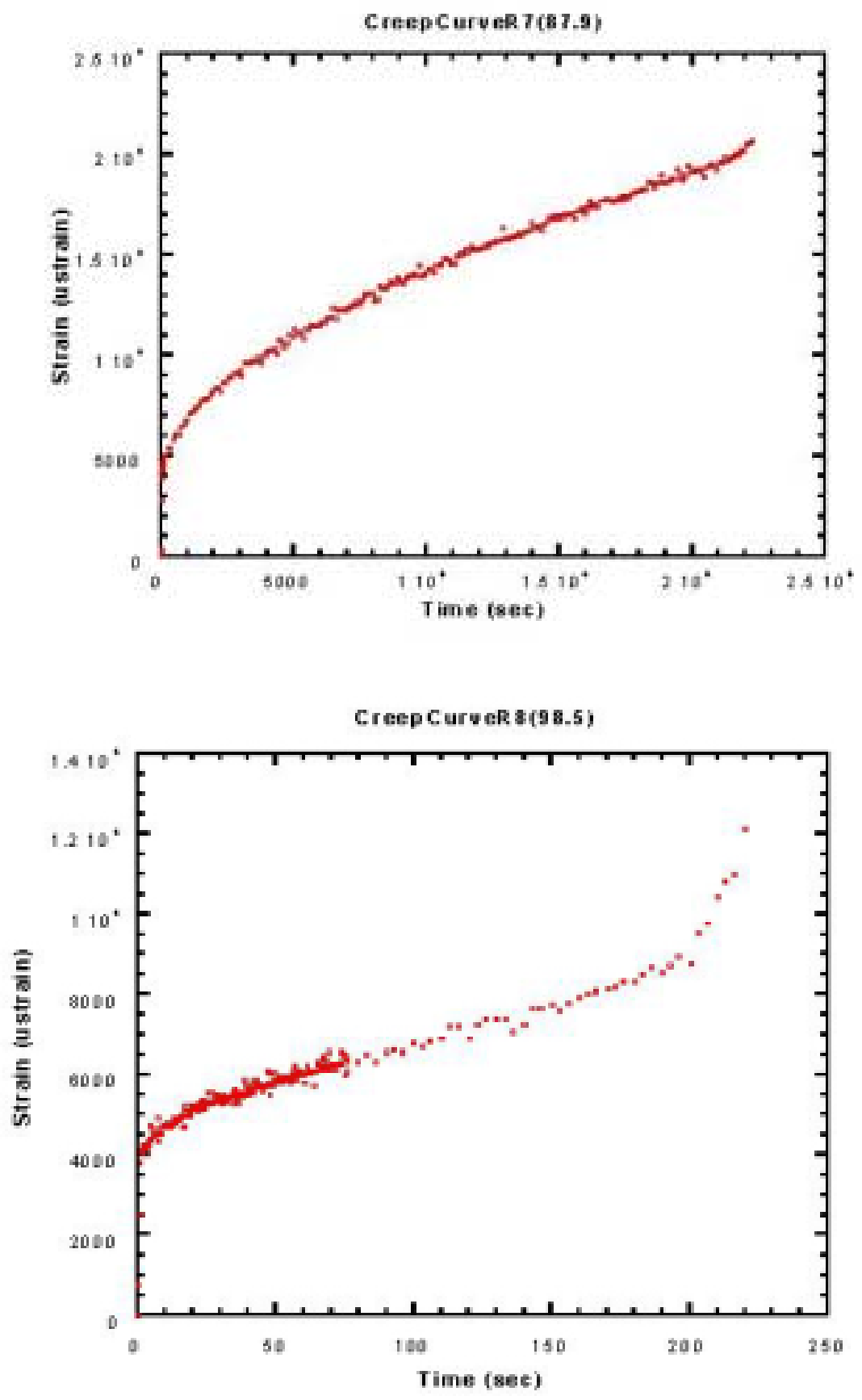

Figure 4.25 Creep curves of specimens R7 and R8, held at 87.9MPa and 98.5MPa, respectively. 
Norton-Enquist creep model was utilized to fit individual creep curves using equation

$$
\varepsilon=A \sigma^{B} t^{C}
$$

where the parameters $A, B$ and $C$ were to be determined from the fit of data. The results, along the times to failure of each of the specimens are summarized in Table 4.4. Figure 4.26 demonstrates an example of creep curve fit with the above function.

Table 4.4. A summary of specimens tested and the corresponding material parameters.

\begin{tabular}{ccccccc}
\hline Specimen \# & $\begin{array}{c}\text { Stress level } \\
(\mathrm{MPa})\end{array}$ & $\mathrm{A}$ & $\mathrm{B}$ & $\mathrm{C}$ & R value & $\begin{array}{c}\text { Time to } \\
\text { Failure } \\
(\mathrm{sec})\end{array}$ \\
\hline R3 & 82.4 & 32.401 & 0.74215 & 0.28479 & 0.924 & 63256 \\
R4 & 86.7 & 57.087 & 0.90515 & 0.18331 & 0.994 & 46814 \\
R5 & 95.9 & 39.241 & 0.95517 & 0.2589 & 0.989 & 499 \\
R6 & 97.1 & 41.85 & 0.91445 & 0.21355 & 0.985 & 285 \\
R7 & 87.9 & 25.356 & 0.67043 & 0.36416 & 0.992 & 22275 \\
R8 & 98.5 & 42.842 & 0.90131 & 0.20945 & 0.916 & 220 \\
\hline
\end{tabular}

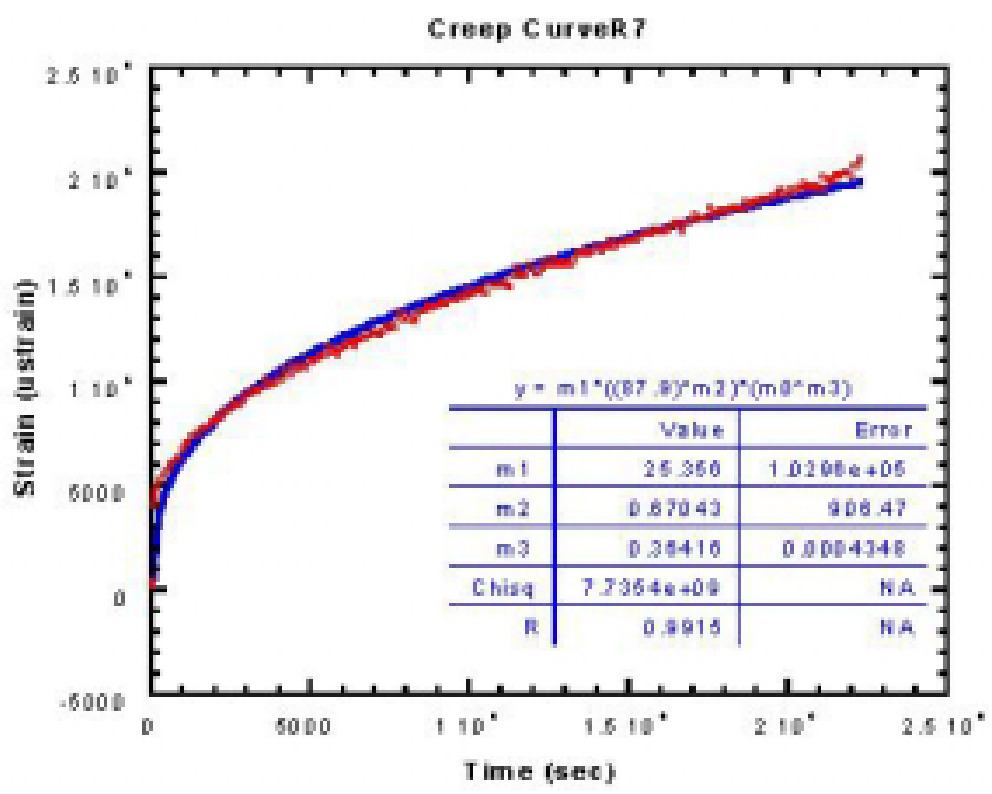

Figure 4.26. Creep curve of specimen R7 fit with Norton-Enquist equation with the resulting parameters. 
After obtaining the necessary data, in particular time to failure corresponding to a range of stresses above the threshold value of stress, we can now proceed in determining the relationship between those variables and hence, determine the last two parameters necessary for the completion of the model. Subsequently, time to failure was plotted against the parameter indicating the difference between the threshold stress and the hold stress. The result is given in Figure 4.27.

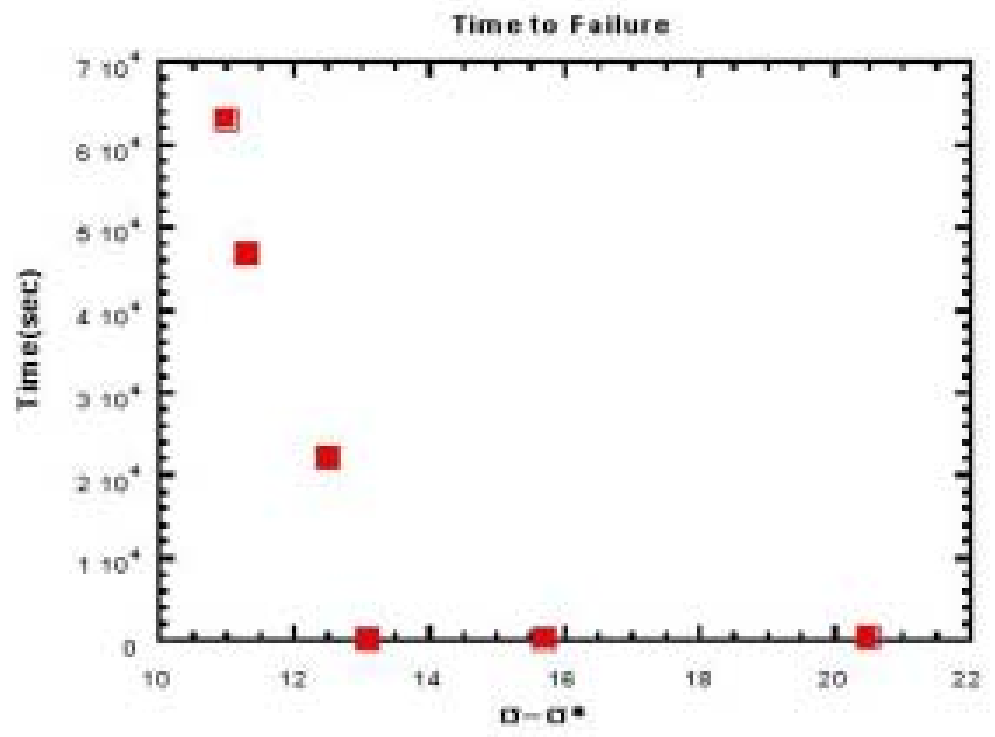

Figure 4.27. A graph representing the relationship between $t_{\mathrm{F}}$ and $\left(\sigma-\sigma_{\mathrm{TH}}\right)$.

Various functions were utilized to fit this relationship. Exponential was the only one with a relatively good fit $(\mathrm{R}=0.72)$. Nevertheless, the best fit was obtained by using the relationship already proposed by Equation (4.66). The resulting plot is given in Figure 4.28. 


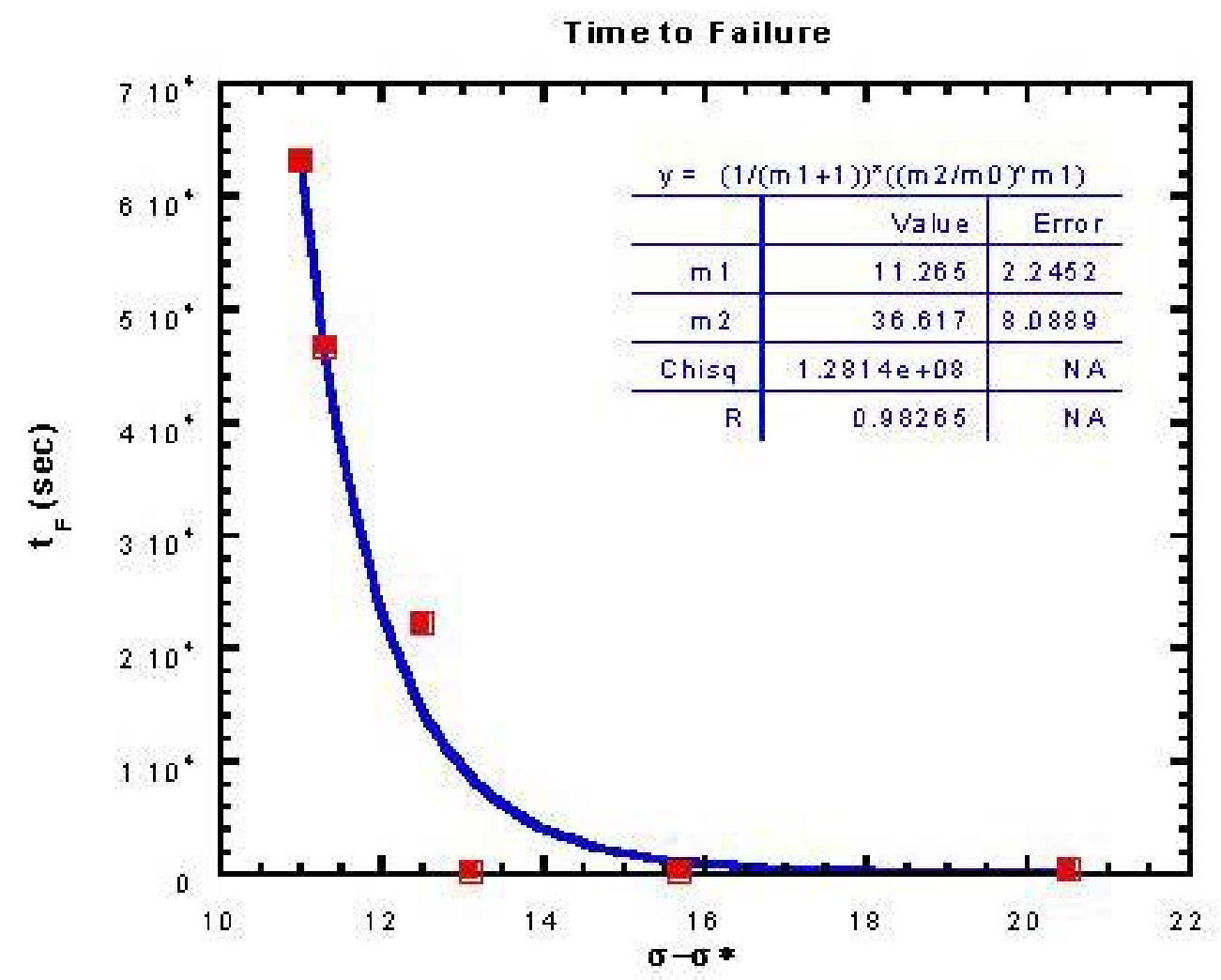

Figure 4.28. A graph representing the fit of time-to-failure vs. hold stress using Equation 4.66

From the parameters obtained from the fit the parameters $r$ and $A_{0}$, necessary for the model were identified as

$$
\begin{gathered}
r=11.265 \\
A_{0}=36.617
\end{gathered}
$$

Now, that we have obtained all the necessary parameters for the Equation (4.73) we can use it to predict the viscoelastic behavior of bone coupled with damage. Keeping in mind that for the stress levels below the threshold the Equation (4.73) becomes Equation (4.74) we can use them to predict viscoelastic strain borne in bone specimens. Also, we can design verification tests in order to compare the predicted strains with the ones measured by the experiment and hence validate the proposed model. 


\subsection{Theoretical Predictions}

In this section we will use the parameters obtained from the experimental studies described in the previous sections to perform numerical experiments. From the results of previous sections parameters are given

$$
\begin{aligned}
& A_{0}=36.617 \\
& r=11.265 \\
& 27.432<J_{0}<46.262 \quad\left(J_{0}\right)_{\mathrm{av}}=37.9034 \\
& 4.621<J_{1}<12.482 \quad\left(J_{1}\right)_{\mathrm{av}}=10.7216 \\
& 0.055<\kappa<0.0929 \quad(\kappa)_{\mathrm{av}}=0.0724
\end{aligned}
$$

Substituting those parameters into Equation (4.73) and using Equation (4.66) we will get

$$
\begin{aligned}
& \varepsilon=37.9034 \sigma\left(1-\frac{0.08153282\left[\frac{36.617}{\sigma-75.29}\right]^{11.265}}{0}\right)^{-0.08153282} \\
& +10.7216 \sigma t^{0.0724} F\left[[1,0.08153282],[1.0724] ; \frac{0.08153282\left[\frac{36.617}{\sigma-75.29}\right]^{11.265}}{t}\right]
\end{aligned}
$$

Equation could be readily evaluated as a function of time for any given value of stress. Maple6 software was used to evaluate the above expression. The code for the evaluation is given below 


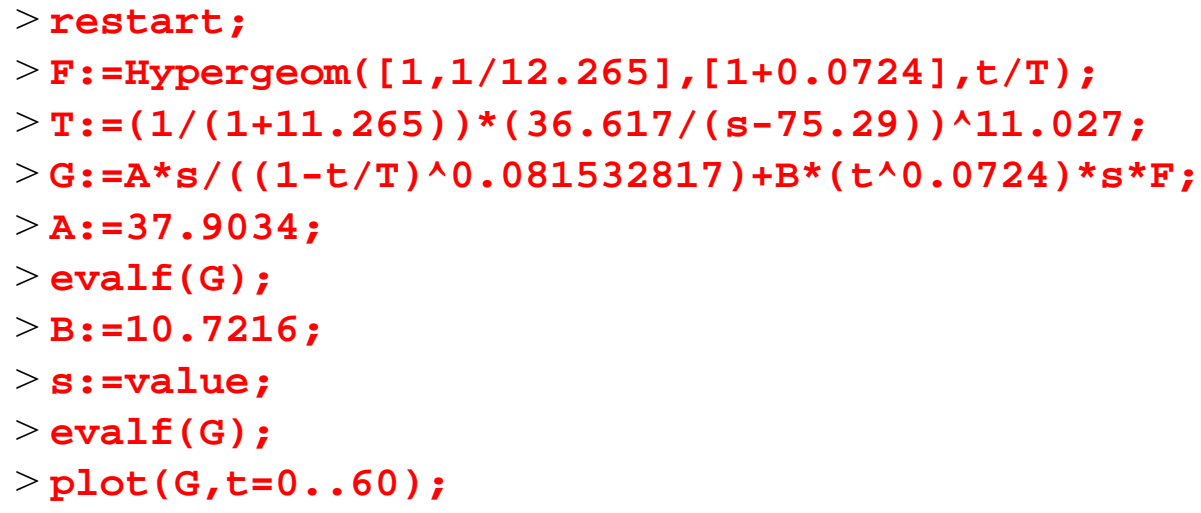

Figure 4.29 represents the result of a simulation for a stress $(\mathrm{s}=80 \mathrm{MPa})$ and for duration of $(t=200 \mathrm{sec})$, where strains have units of microstrain.

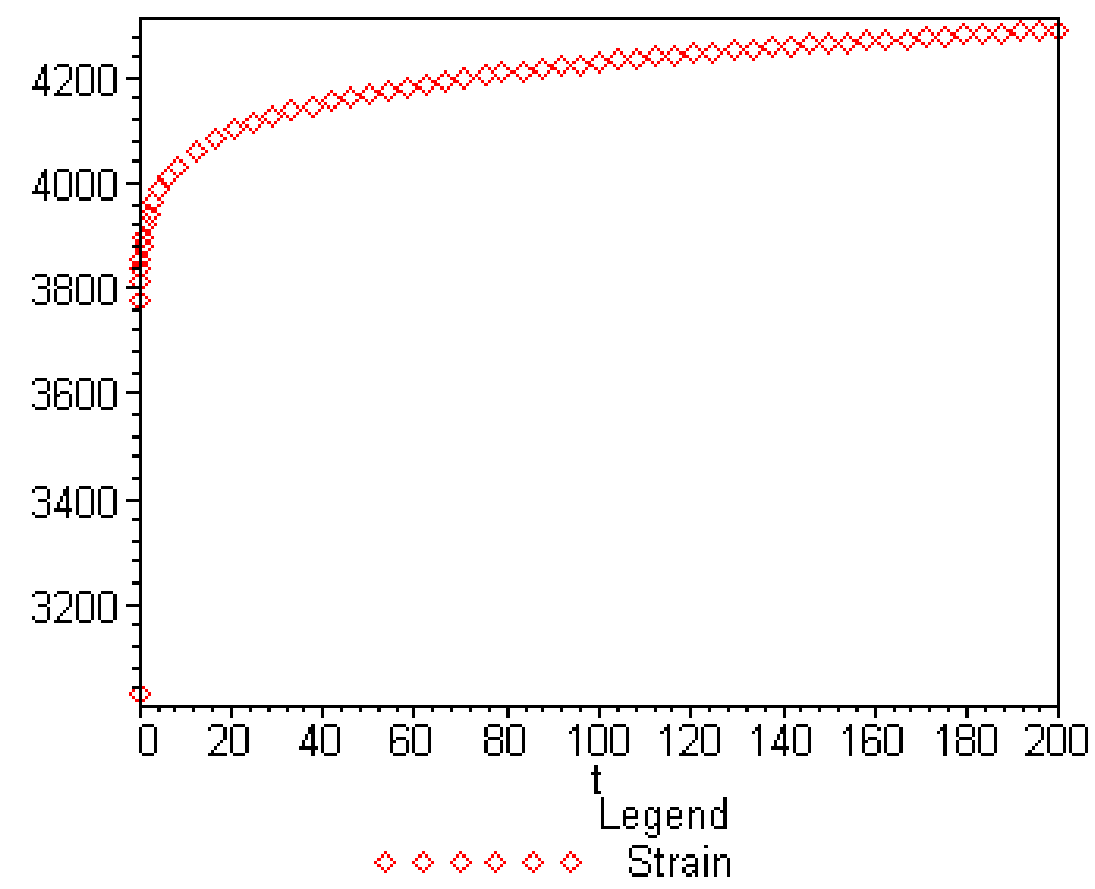

Figure 4.29. Strain prediction for $\sigma=80 \mathrm{Mpa}$ and $\mathrm{t}=200 \mathrm{sec}$.

It is important to demonstrate that the series defined by the Hypergeometric function converge for $0<\mathrm{t}<\mathrm{t}_{\mathrm{F}}$, hence predict the failure. This could be demonstrated by setting the stress equal to a value very close to the threshold stress and performing the simulation for an infinite amount of time. Theoretically, it should predict the failure. This procedure was carried out for stress $=75.3$ and the result is plotted in Figure 4.30. 


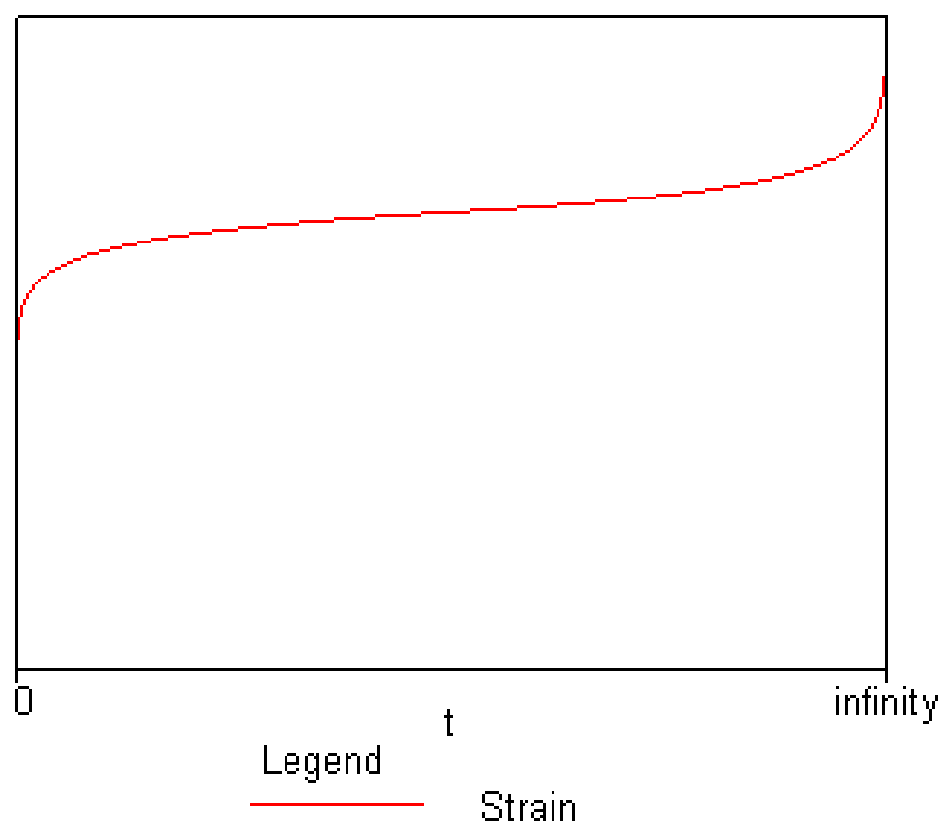

Figure 4.30. Strain prediction at the stress value approximately equal to the threshold value demonstrating the tertiary creep stage and hence the convergence at the infinity.

Figures 4.30, and 4.31 demonstrate results of various simulations, clearly indicating the ability of the model to predict the characteristic creep response and failure. In the Figure 4.30 results of series of simulations are plotted leading to the prediction of failure at the stress level of $85 \mathrm{MPa}$.
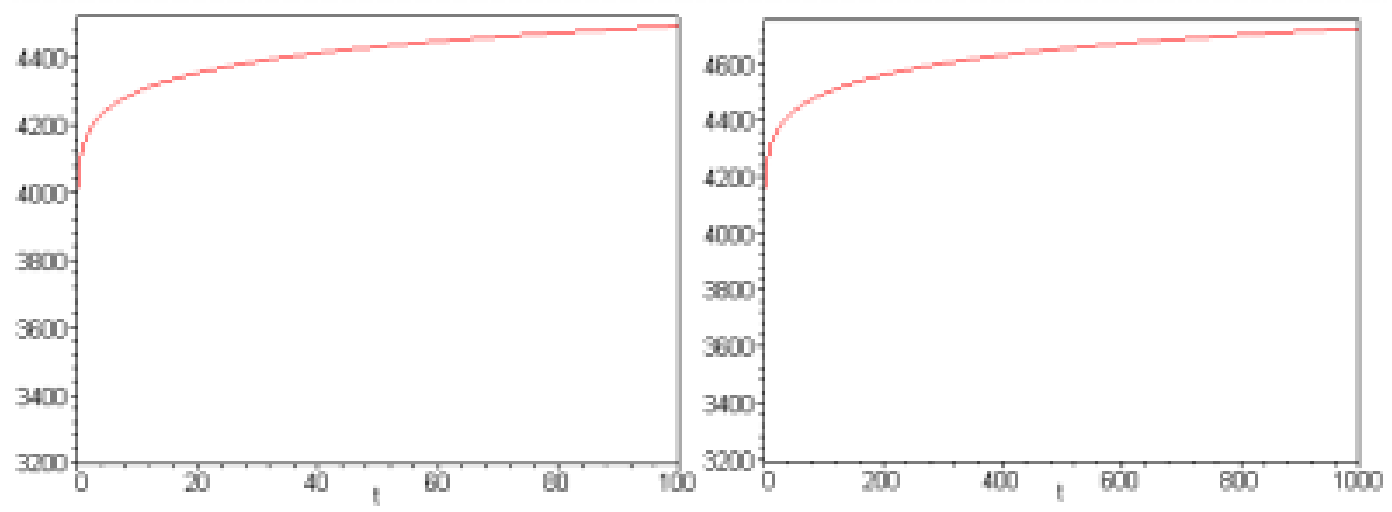

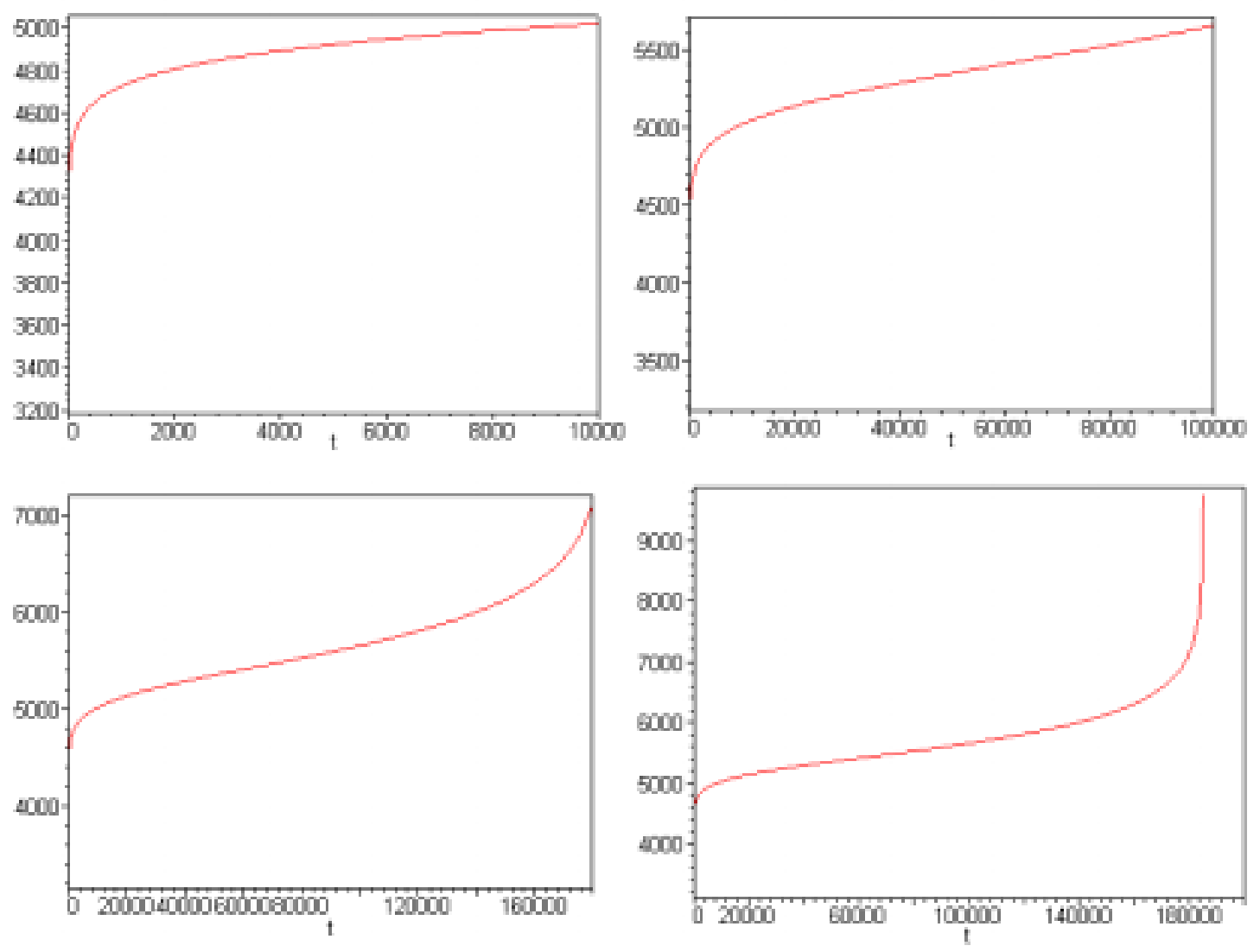

Figure 4.30. Series of simulations at the $\sigma=85 \mathrm{MPa}$.

These simulations indicated that at $85 \mathrm{MPa}$ stress level the specimen should fail after approximately 180000seconds. Experimental results from the specimen (R4) tested at $86.7 \mathrm{MPa}$ yielded $\mathrm{t}_{\mathrm{F}}=46814 \mathrm{sec}$. To test which of the parameters is responsible for underestimation of $t_{\mathrm{F}}$ we performed series of simulations using different variables from the range given earlier. Prior to performing those simulations we examined the equation for strain more closely in order to have an intelligent guess which of the parameters is responsible for the underestimation. $J_{0}$ should not play a significant role as its' variation would lead only to the change of the initial strain. $J_{l}$ is multiplied by a faster growing function and hence its' role in causing an increase in the slope of the curve would not be significant. Any change in the value of $\kappa$ would ultimately lead to the change of the slope, since it is the power in the function of time, obtained from strain vs. time fits. The higher the $\kappa$ the further away from the time axis the curve will move, resulting in an increase in strain rate. Lastly and most importantly the parameters obtained from the $t_{F}$. data would have the biggest influence on the behavior of this model. Since we had data 
only from six specimens which ranged from 200 to 64000 seconds with practically no intermediate points, this would lead to a fit with a very sharp neck region and hence to very fast growing function, which in turn would lead to large changes of variable y with a slight change of variable $\mathrm{x}$. To test the influence of variable $\kappa$ we performed simulations by varying $\kappa$ from its' minimal value of 0.055 to its' maximum value of 0.0929 . The results are given below, verifying our early speculation that it would increase the slope of the curve but would not necessarily lead to better estimation of $t_{F}$.(Figure 4.31).
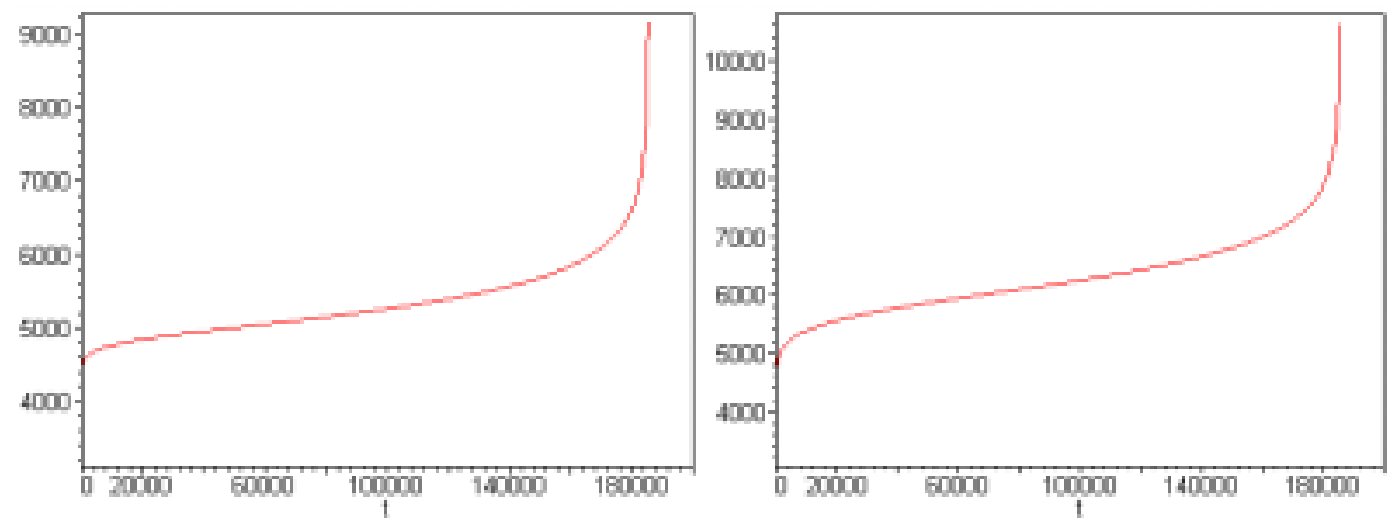

Figure 4.31. Predicted strain at $\sigma=85 \mathrm{MPa}$ with $\kappa=0.055$ and $\kappa=0.0929$

Similar analysis were carried out at 100MPa stress, leading to an estimated failure within a short period of time, in this case within 6 seconds (Figure 4.32)
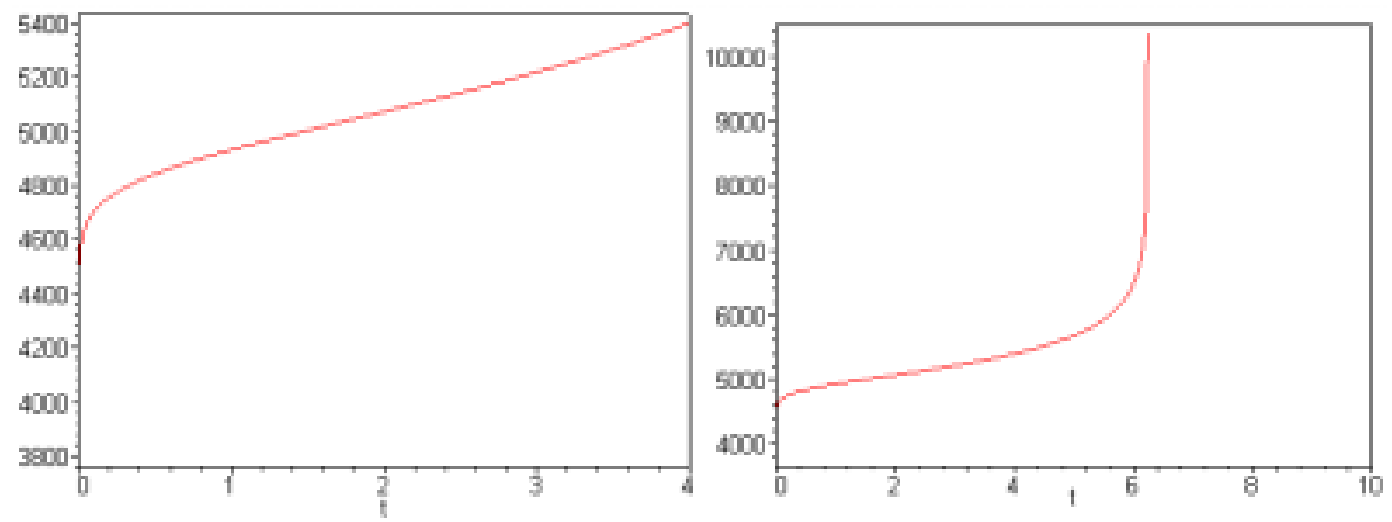

Figure 4.32. Predicted strain at $\sigma=100 \mathrm{MPa}$. 
To further demonstrate the advantages of this model we will proceed to demonstrate the behavior predicted using Equation (4.74), which does not include influence of damage. Figure 4.33 demonstrates results obtained by predicting the strain using Equation (4.74) at stress levels 50,60 and 70MPa. The Maple code is given below.
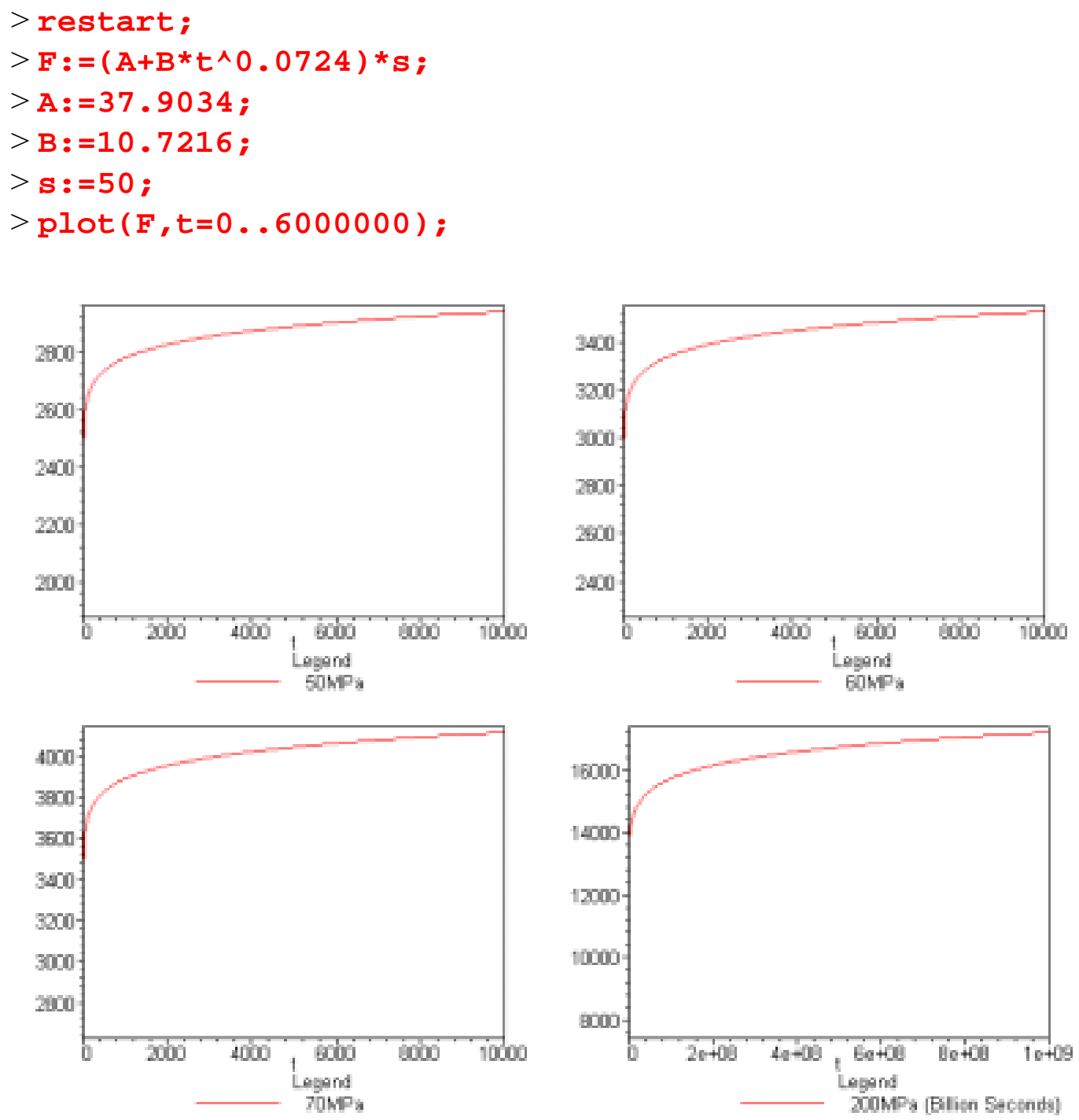

Figure 4.33. Series of simulations using Equation 4.74 at 50,60 and $70 \mathrm{MPa}$ and at $\sigma=100 \mathrm{MPa}$ for a long duration of time demonstrating inability to predict tertiary creep and failure.

In order to illustrate that change in $\kappa$ leads to a change in slope two simulations were run for the same stress level and the same duration, and with two extreme values of 
$\kappa$ The results are given in Figure 4.34, which clearly indicate the change of the slope in the creep curves.
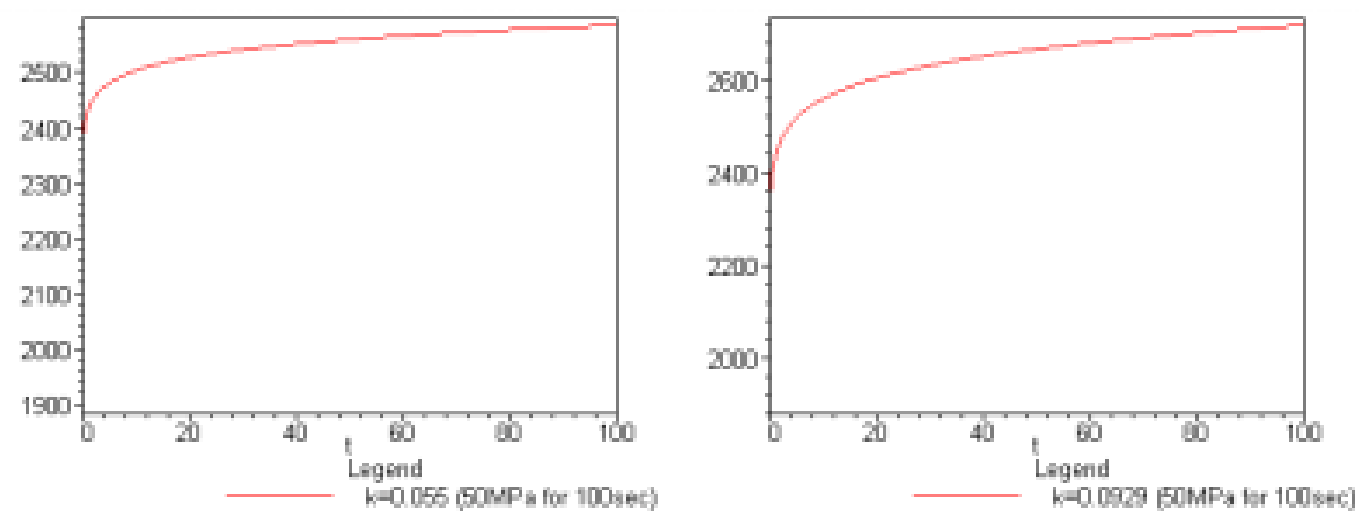

Figure 4.34 simulation at identical loading conditions demonstrating the influence of $\kappa$

Finally to demonstrate the validity of Equation 4.75 to describe the recovery behavior, it was evaluated for several load levels and time durations. The results are given in Figure 4.35 .
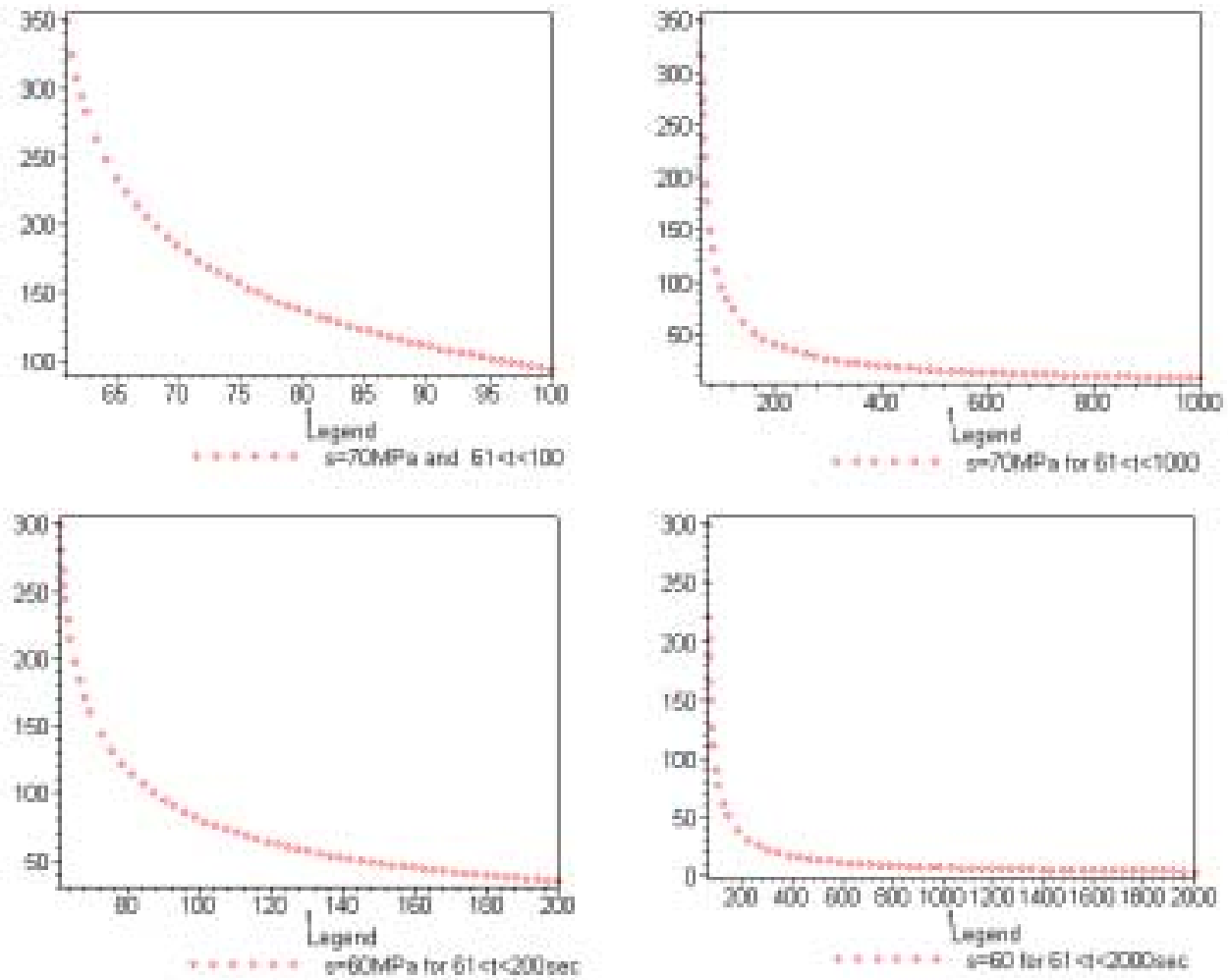
Figure 4.35 Strain recovery prediction using Equation 4.75 after hold stress of 70MPa for 60 seconds (plots 1 and 2) and hold stress of 60MPa for 60 seconds (plots 3 and 4).

\subsection{Validation of the Model}

\subsubsection{Experimental Methods}

In this portion of the study several specimens (group $\mathrm{V}(\mathrm{n}=6)$ ) machined from another section of the same tibia would be tested in order to compare the predicted strains with the observed strains. The experimental protocol for specimens V1-V3 consisted of two cycles. During the first cycle a load ramp would be applied to a load level below the threshold and held for 60 seconds followed by another load ramp above the threshold and a hold until failure. Specimen V1 was not held until failure during the $2^{\text {nd }}$ cycle, whereas specimens V2 and V3 were tested until failure. Specimen V4 was tested at a low stress level (just above the threshold) until failure.

\subsubsection{Results and Discussion}

Figure 4.36 demonstrates the strain history during the initial ramp applied to the specimen V1 below (54MPa) the threshold value of stress. The solid line represents the predicted strains using the Equation 4.74. It could be seen that within the error of the initial strain the predicted strains closely match the observed ones. One possible reason for the mismatch lies within the large scatter of results for the determination of parameters $J_{0}, J_{1}$ and $\kappa$, obtained in the section 4.7 , which are utilized here for the prediction of strain. 


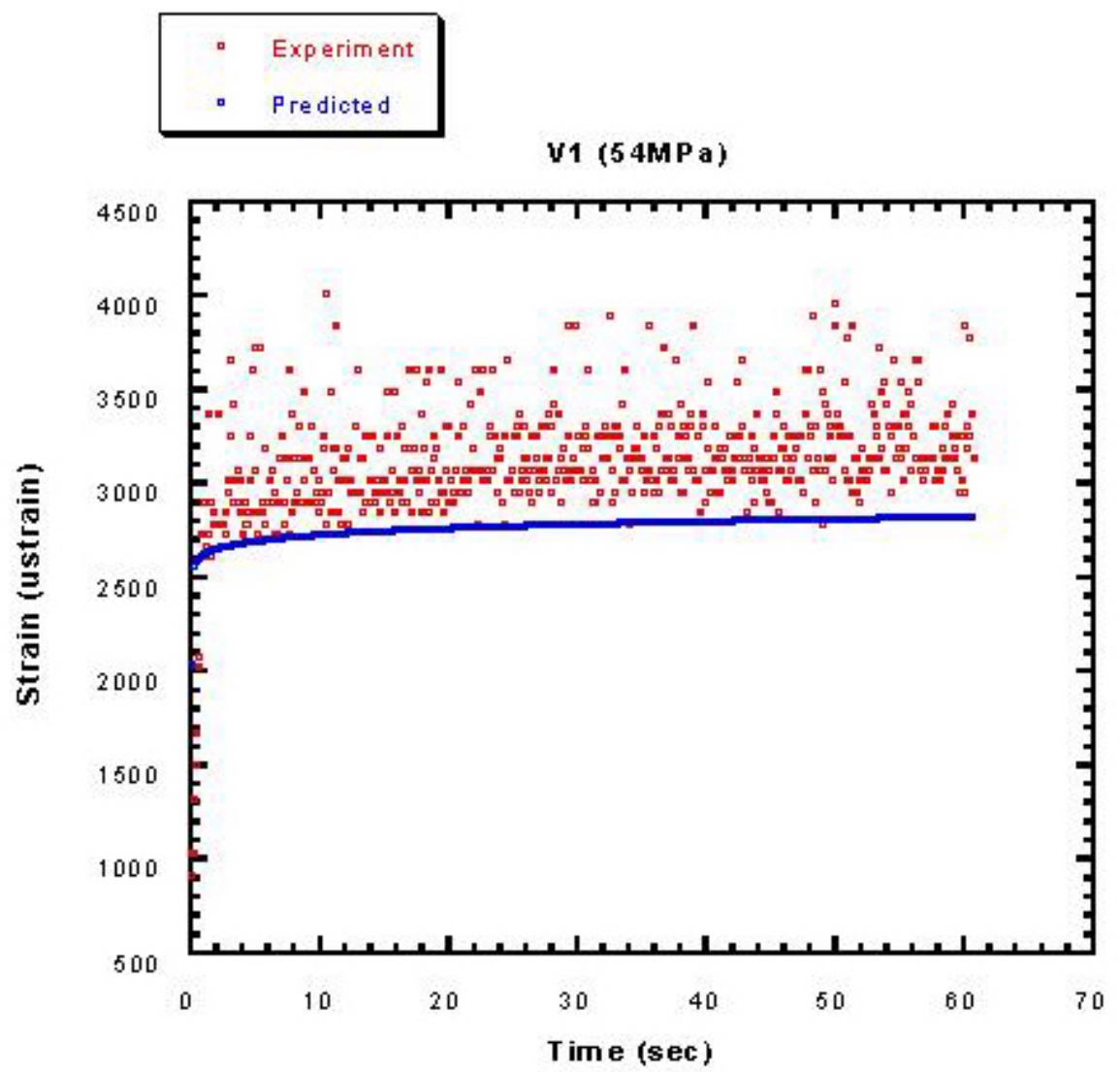

Figure 4.36 A graph representing the observed and predicted strains for specimen V1 held at 54MPa.

Figures 4.37 and 4.38 represent the observed and predicted strains of specimen V1 held at $82.4 \mathrm{MPa}$. 


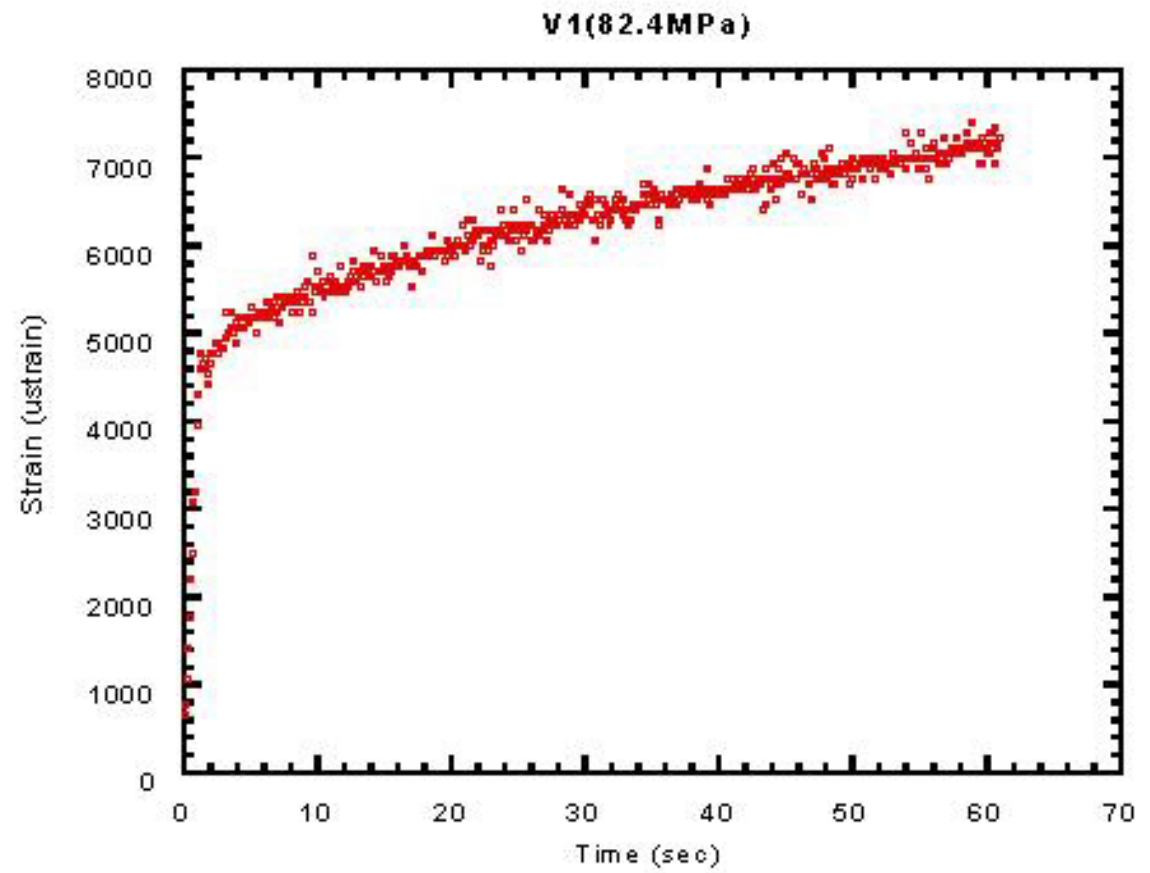

Figure 4.37 Experimental strain versus time for specimen V1 held at 82.4MPa for 60 seconds. 


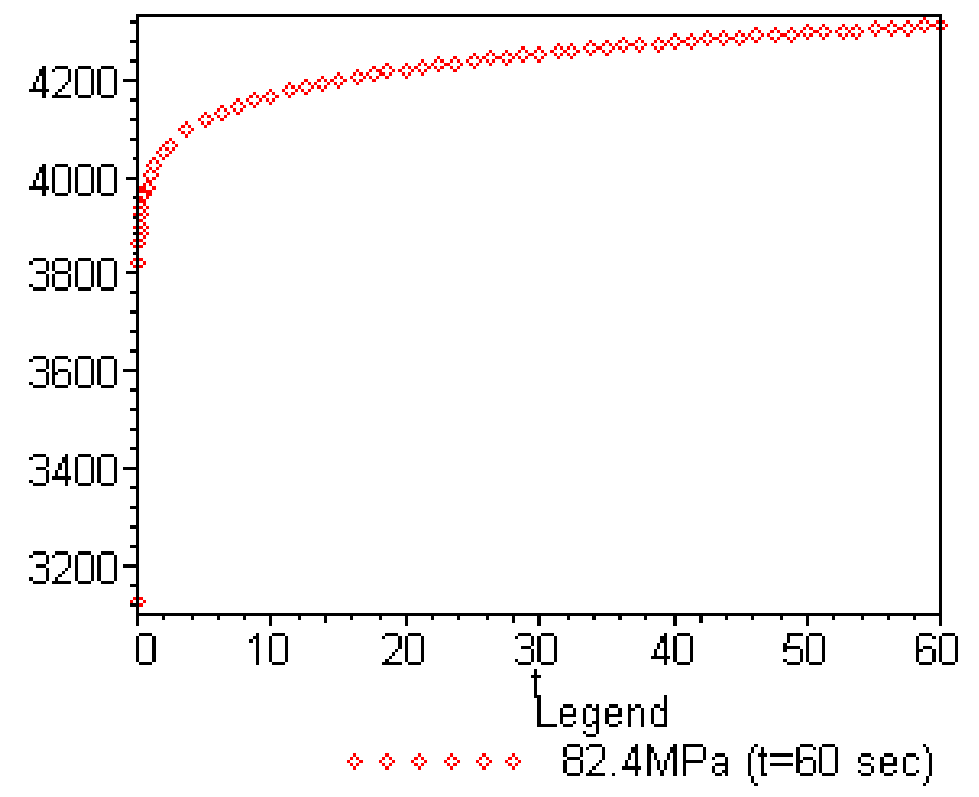

Figure 4.38 Strains predicted by the model for a specimen held at $82.4 \mathrm{MPa}$ for 60 seconds.

By comparing those plots, one obvious observation is that the model does not accurately predict the strain rate observed in the experimental results. It could be seen from the graph in Figure 4.37 that during the hold period of 60 seconds strain increases by approximately $2000 \mu$ strain, whereas the predicted strains increase by approximately $600 \mu$ strain. There are several possible scenarios that could be responsible for this behavior. We would discuss all the scenarios after studying the model's ability to predict the time of failure.

Figure 4.39 demonstrates the observed and predicted strains of the model for the specimen V2 held at the stress $62.7 \mathrm{MPa}$ for 60 seconds. And Figures 4.40 and 4.41 demonstrate the observed and predicted strains to failure at the subsequent cycle when the specimen was held at $91.7 \mathrm{MPa}$ until failure. In Figure 4.41 the results of series of simulations are plotted in order to demonstrate the initiation stage of tertiary creep and subsequent time of failure. This clearly demonstrates the ability of model to incorporate the initiation of the tertiary stage of creep, caused by the accumulation of damage and hence predict the failure. 


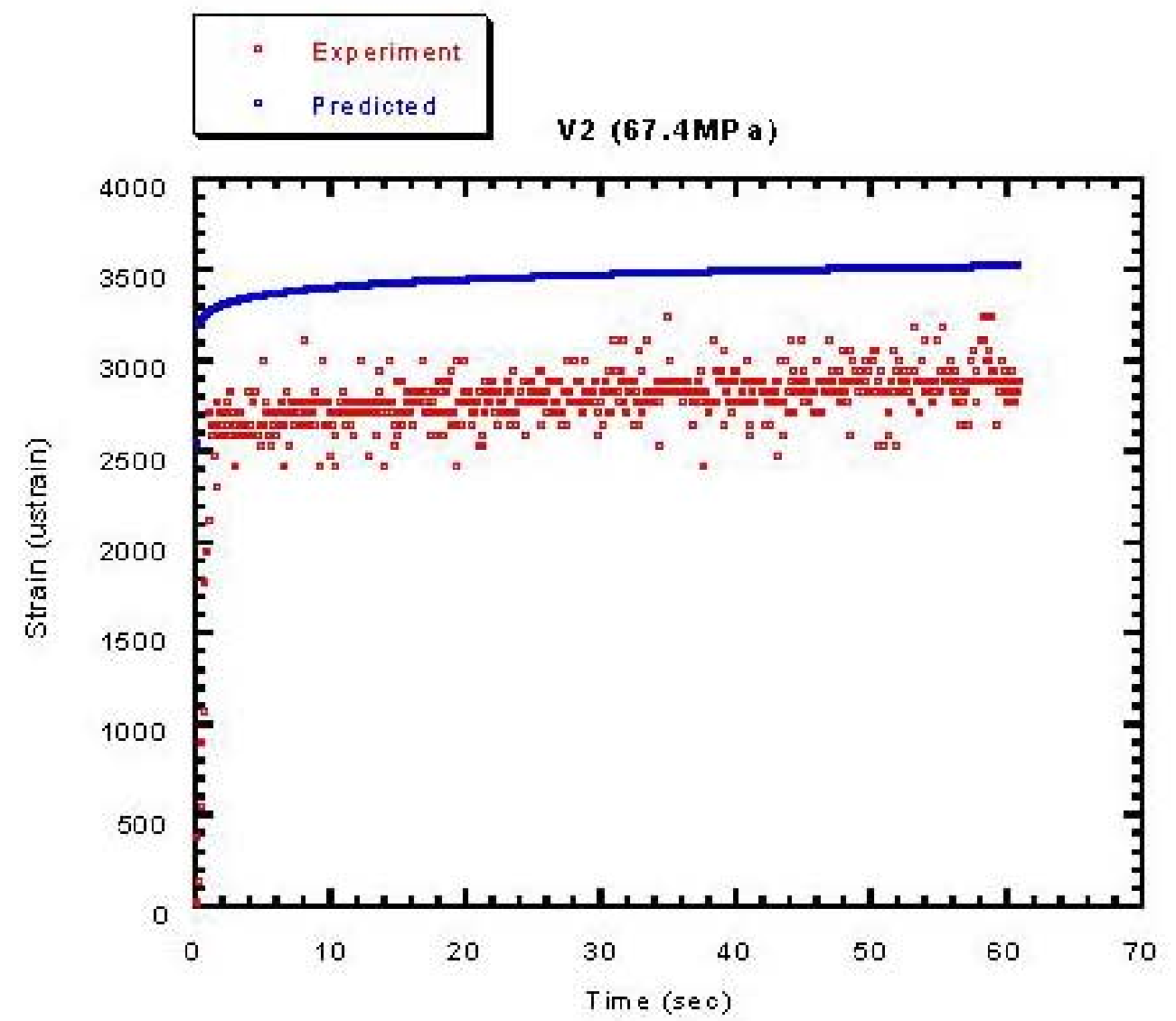

Figure 4.39. Strain versus time history observed and predicted for the specimen V2 at the hold stress level of 67.4MPa and duration of 60 seconds. 


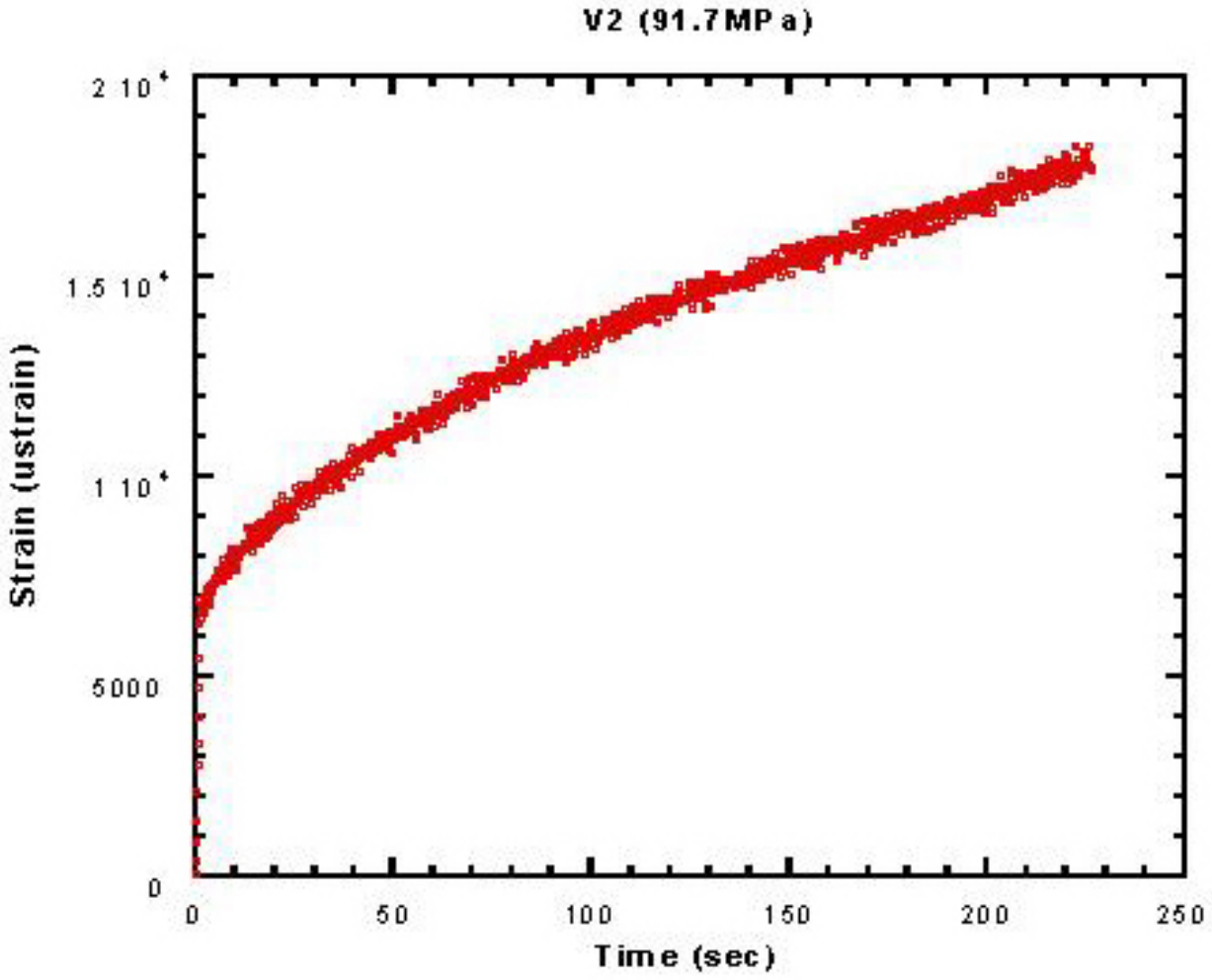

Figure 4.40. Strain history until failure observed for specimen V2 held at 91.7MPa. 

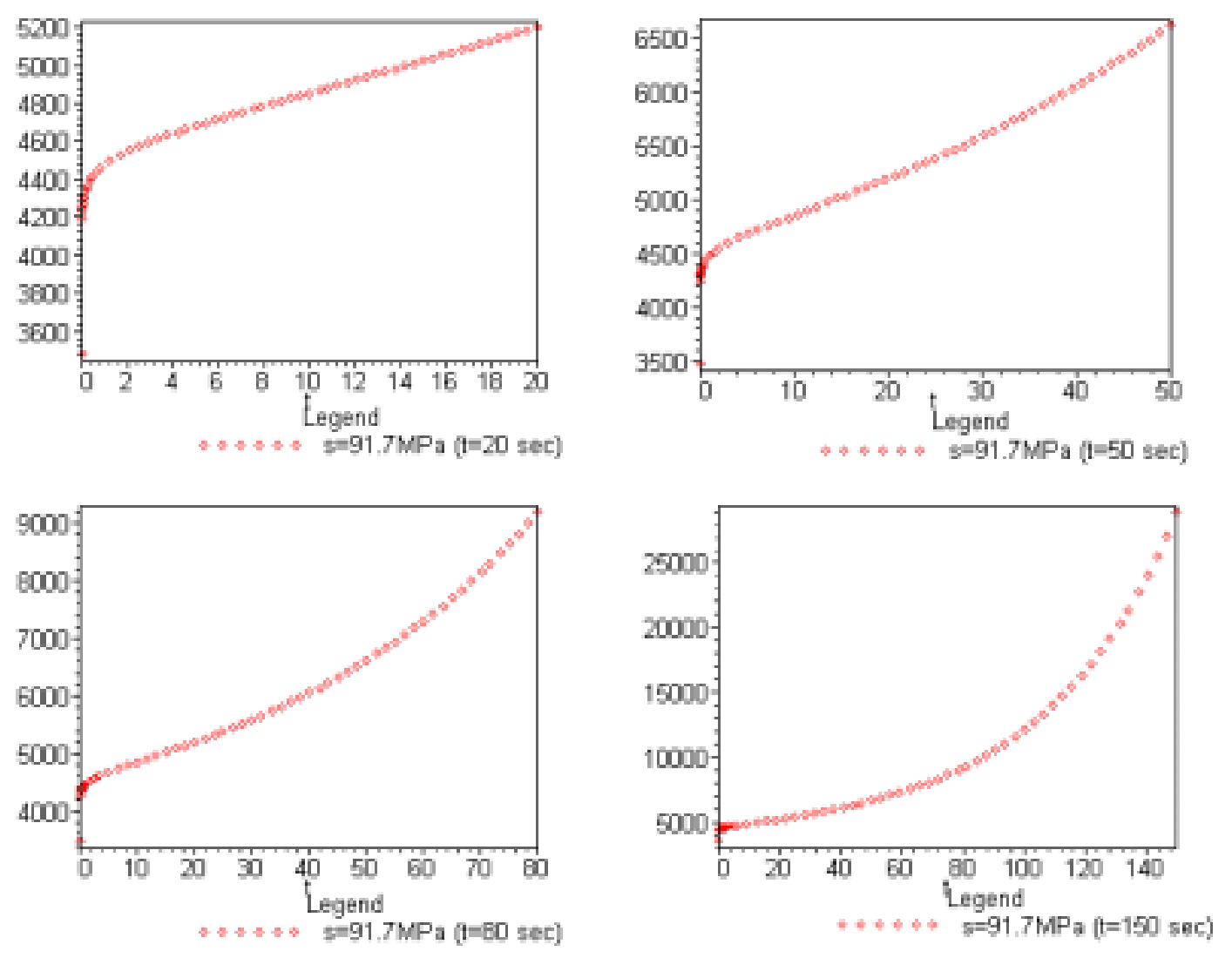

Figure 4.41. A series of simulations for the prediction of failure for a stress level of 91.7MPa.

Figure 4.41 demonstrates that predicted time to failure is in approximately 150 seconds and in the view of the experimentally obtained time to failure of 226 seconds we conclude that in this particular case theoretical predictions closely match the experimental observations.

Similar to the loading protocol for specimen V2, specimen V3 was tested at different prethreshold and postthreshold levels. The results along with the results of simulations are plotted in Figures 4.43-4.45. Results, once again demonstrate good correlation between the model predictions and experimental results. 


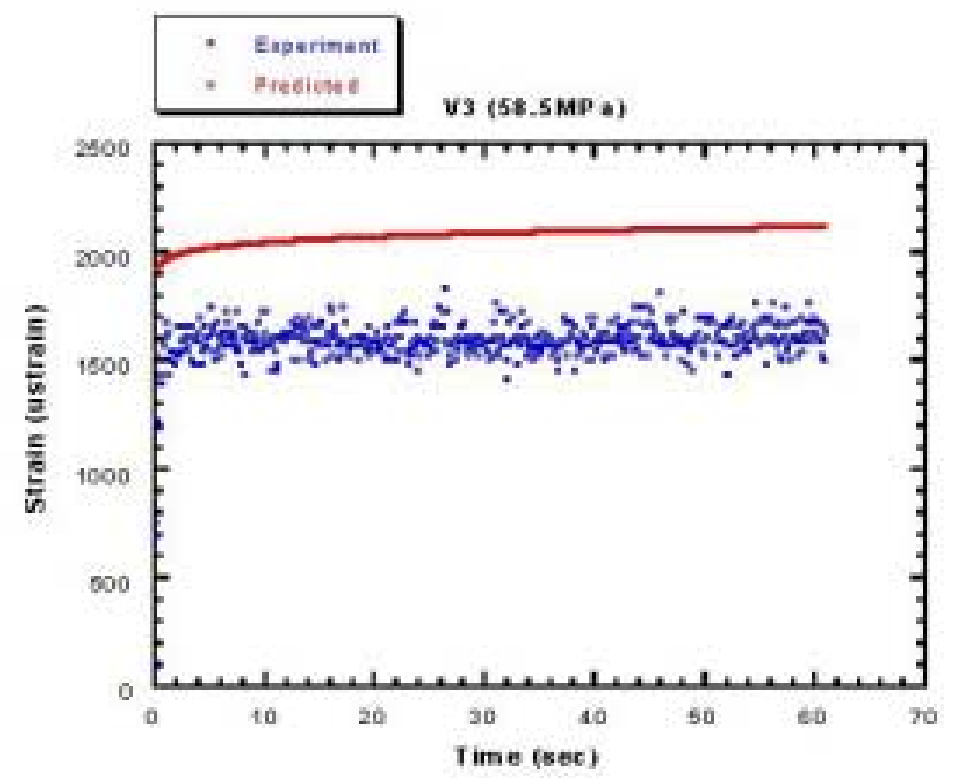

Figure 4.43 Strain versus time history observed and predicted for the specimen V3 at the hold stress level of $58.5 \mathrm{MPa}$ and duration of 60 seconds

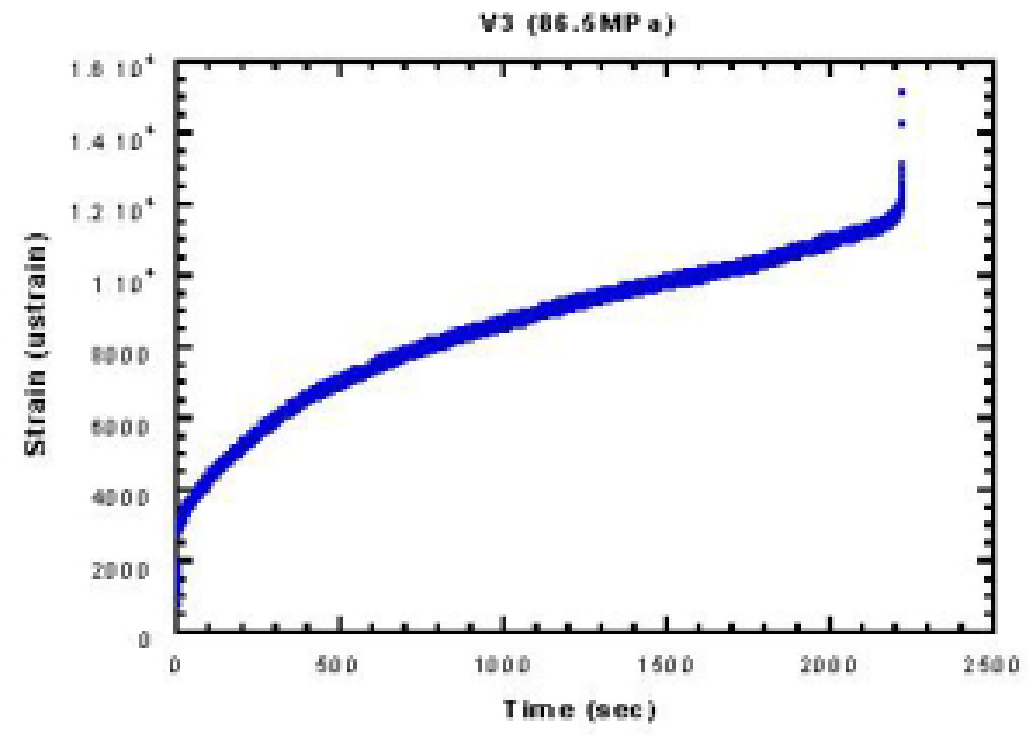

Figure 4.44. Strain history until failure observed for specimen V3 held at $86.5 \mathrm{MPa}$ 

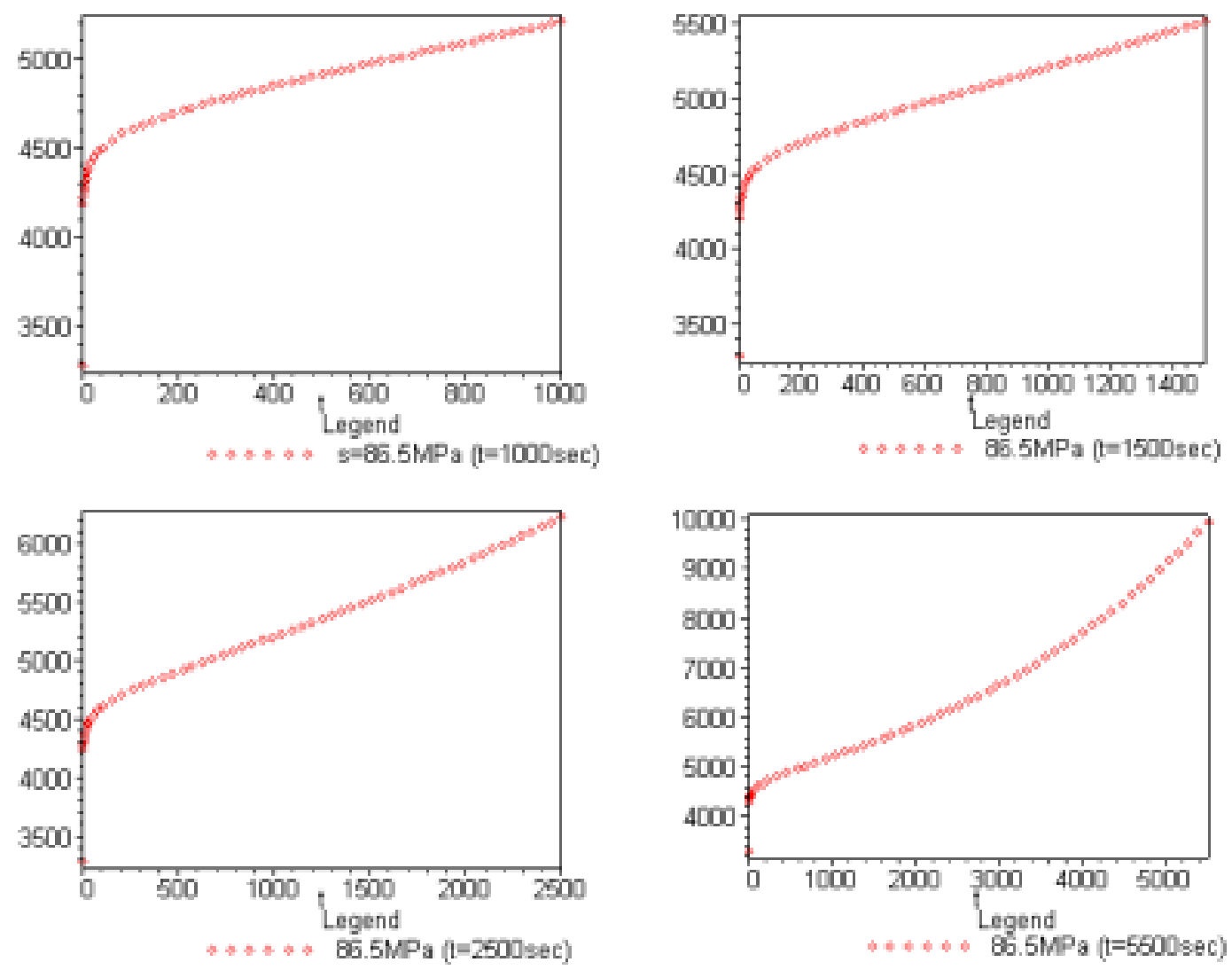

Figure 4.45 Series of simulations for the prediction of failure for a stress level of 86.5MPa.

In order to demonstrate how the variation of parameters obtained in previous section would influence on the ability of the model to predict the experimental creep behavior upper and lower bounds of predictions were defined using the average plus and minus the standard deviation of parameters $J_{0}, J_{l}$ and $\kappa$, respectively (see Table 4.3). Subsequently, simulations were performed at the stress level of $88.4 \mathrm{MPa}$, which was the hold stress level of the specimen V4. Figure 4.46 represents the experimental strain behavior together with model predictions using the upper and lower bounds of parameters. It could be seen that experimental curve lies within the bounds of predicted strains. In addition it could be seen that for both cases; upper and lower model predicts the time to failure with a moderate accuracy. 


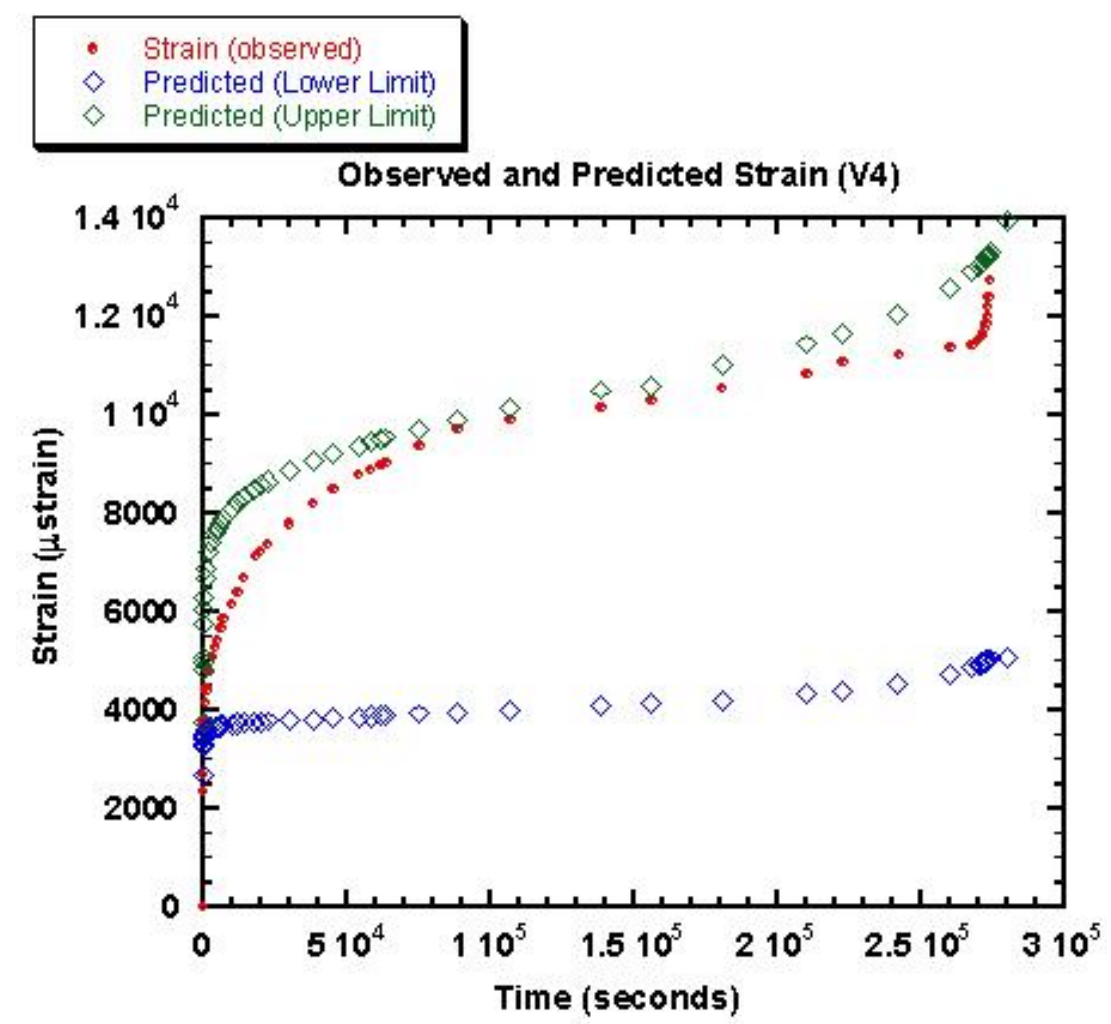

Figure 4.46. A graph representing the strain history observed for the specimen V4 tested at $88.4 \mathrm{MPa}$ until failure together with predicted strains using the upper and lower bounds of parameters $J_{0}, J_{l}$ and $\kappa$.

\subsection{Limitations of the Model}

Although the model proposed in this portion of the study demonstrated good correlation with experimental data it is important to highlight the limitations of this model. The most significant limitations arise form the number of specimens tested in the determination of key parameters of the model. Considering the results of the section that was concerned with the determination of the threshold value of stress we have found a very rapid transition from initially viscoelastic behavior with strain rates of the order 2$3 \mu \mathrm{strain} / \mathrm{sec}$ to strain rates up to $100 \mu \mathrm{strain} / \mathrm{sec}$. As a result of this rapid transition viscoelastic compliance parameters, specifically the parameter indicating the power relationship with time, caused considerable underestimation of strains at failure. Secondly, the power relationship between the time-to-failure and the stress included points only from the extreme ends of the material behavior, namely, rapid failure within 
hundreds of seconds and no failure for an order of days. Obviously, more representative data, which would include the intermediate behavior would be extremely beneficial for more accurate estimations obtained by the proposed model. Thirdly, all the specimens of this study were obtained from a single tibia, hence in order to generalize that the proposed model would be able to predict the viscoelastic damaging behavior of the cortical bone as a material, statistically representative sample of specimens must be used obtained from different donors and from different types of long bones (tibia, femur and fibula). Nevertheless, the proposed model enabled us to demonstrate the onset of the tertiary stage of creep, which is subsequently followed by failure. Therefore it allowed us to predict the influence of damage on the material behavior.

\subsection{Conclusions}

In this portion of this study a continuum damage model was developed for human cortical bone, based on the fundamental thermodynamic principles. Subsequently it was simplified for a simple one-dimensional case and was shown to predict experimental observations with a relative accuracy. The significant shortcomings arose from the fact that group of specimens that were used to obtain key parameters for the model was small leading to high values of deviation from the average values of those parameters. These in turn lead to under or overprediction of strains observed experimentally. Also, in the derivation of the formulation the influence of permanent strains were neglected leading to underestimation of creep induced permanent deformations. To our knowledge this is the first model developed in the field of bone mechanics that encounters the time-dependent and nonlinear processes in this material, simultaneously. The developments of this chapter serve as a good foundation for further validation of the model and, perhaps, implementation into Finite Element packages as a more accurate constitutive formulation

of bone as a material. Furthermore, a more accurate FE model of bone would ultimately be very beneficial in design and studying various orthopedic implants. 


\section{CHAPTER 5}

\section{CONCLUSIONS AND FUTURE DIRECTIONS}

The overall contribution of this work for the current bone research could be summarized as follows. In Chapter 2 we have demonstrated technique in studying various stages of damage development in bone using Laser Scanning Confocal Microscopy. Namely, by using compact tension specimen geometry we were able to monitor stages of damage development in the damage process zone ahead of the crack tip induced by low stress level cyclic loading. The geometry of the specimens, which allowed localization of those damaging processes into the area of the crack tip together with high resolution microscopy, such as confocal microscopy proved to be an excellent technique to monitor damage development. More importantly, allowed us to identify the early stages of damage and the microarchitectural sites involved. In addition by controlling the amount of damage induced in the Damage Process Zone of the specimens we were able to address the question of the influence of submicroscopic diffuse damage on fracture toughness and found that diffuse damage causes toughening-type phenomenon to occur. Overall observations have led us to suggest an alternative scenario of damage induced bone remodeling. Namely, it is possible that alterations of the strain environment at the local level of osteons would cause mechanosensing octeocyes to trigger intracortical remodeling response carried out by bone lining cells housed on the bone surfaces within blood channels. This could possibly explain the evidence of constant remodeling processes demonstrated by the formation of secondary osteons throughout life without an extensive evidence of large microcracks. In the context of the importance of understanding the mechanisms responsible for skeletal fragility and various debilitating bone diseases it makes the findings of this study important not only from the aspect of materials science but also from the biological prospective. In terms of future directions, it would be interesting to investigate the influence of bone morphologic parameters on the results obtained in Chapter 2. Regarding how to test weather our suggestions about the biological responses are correct, I suggest the following experiment. First design an animal model: a specific fixture in order to apply loading on tibia (femur) of rats with ultrasonic sensors attached on the bone surfaces. Perform sets of experiments in order to 
correlate the events recorded by ultrasonic sensors with the results of histology. This procedure would demonstrate what the ultrasonic events detected are associated with, therefore validate that damaging events can be detected. In addition the detailed histology, preferably using LSCM, would also allow investigating the type of damage (submicroscopic (diffuse) cracking versus appearance of large microcracks). After validating that this method, long term experiments could be designed and carried out in order to study weather biological responses are associated with various amounts of induced damage at various length scales.

In Chapter 3 we extended the work in Chapter 2 to study the nature of damage in a more complex 3-point bending loading environment. We were able to address the issues regarding the influence of shearing stresses on the overall failure behavior by varying the aspect ratios of specimens tested. Also by utilizing a loading protocol, which involved unloading after every loading cycle we were able to study progressive damage development. As a result evolutionary equation was obtained describing the stiffness behavior and the behavior of permanent strains were also investigated. The relationship between the stiffness drop and the corresponding nonlinear strains were quadratic. Permanent strains demonstrated a constant relationship with the total strains which remained the same throughout all the cycles. These relationships could readily used for the development of a model, which would predict time-independent material response under low-cycle fatigue conditions.

In Chapter 4 a constitutive model was proposed based on thermodynamics of irreversible processes. The experiments demonstrated that bone behaves like an viscoelastic-damaging material. The final predictions of the model demonstrated overall validity of the model and made the shortcomings of it clearer. For simplification purposes the model was derived considering only two phenomena, namely viscoelasticity and damage. As a result upon the removal of the external loads model did not predict any residual effects as both the viscoelastic and damage induced strains recover. As the experiments demonstrated at high stress levels $(>90 \mathrm{MPa})$ bone undergoes considerable amount of creep deformation and since the model does not include the deformations associated with this behavior it leads to underestimation of total nonlinear strains observed during the experiments. In terms of future directions the shortcomings of the 
proposed formulation should be addressed one-by-one. First, parameters obtained for the model must be determined from larger statistical sets of data. Second, permanent deformation associated with creep and creep-damage should be incorporated into the derivation of the total strain for more accurate predictions. This task could be done by utilizing well-developed general formulations for this behavior developed for other engineering materials. In summary, the proposed formulation could be implemented in FE codes for more accurate description of bone response to static loading and hence enhance the accuracy of prosthetic implant design and evaluation using FE software. 


\section{CHAPTER 6}

\section{REFERENCES}

Abdel-Tawab, K. and Weitsmann, Y. 1998. A coupled viscoelasticity/damage model with application to swirl-mat composites. Int .J. Damage Mech. 7:351-380.

Akhter, M.P., Taber, B.J., Kimnul, D.B. and Recker, R.R. 1993. Demonstration of fatigue damage in rat tibia. Trans. Orth. Res. Soc.

Anderson, J and Eriksson, C. 1968. Electrical properties of wet collagen. Nature 218:166-168.

Anderson, J and Eriksson, C. 1970. Piezoelectric properties of dry and wet bone. Nature. 227:491-492.

Ascenzi, A; Bonucci, E. and Bocciarelli, D.S. 1967. An electron microscope study on primary periosteal bone. J. Ultrastr. Res. 18:605-618.

Ascenzi, A; Bonucci, E; Ripatomi, A and Roveri, N. 1978. X-ray diffraction and electron microscope study of osteons during calciffication. Calc. Tiss. Res. 25:133-43.

ASTM Standards E399-83. Standard test method for plane strain fracture toughness of metallic materials. 1985:03.01.

Barrow, G.W. and Saha, S. 1988. Menstrual irregularities and stress fractures in collegiate female distance runners. Am J. Sports Med. 16:209-216.

Becher, P.F. 1991. Microstructural design of toughened ceramics. J. Amer. Ceram. Soc. $74: 255$. 
Behiri, J.C. and Bonfield, W. 1980. Crack velocity dependence of longitudinal fracture in bone. J. Mat. Sci.15:1841-1849.

Behiri, J.C. and Bonfield, W. 1984. Fracture mechanics of bone -the effects of density, specimen thickness and crack velocity on longitudinal fracture. J. Biomech. 17:25-34.

Bentolila, V., Boyce, T.M., Fyhrie, D.P., Drumb, R., Skerry, T.M. and Schaffler, M.B. 1998. Intracortical remodeling in adult rat long bones after fatigue loading. Bone. 23:275281.

Biot, M.A. 1941. General theory of three-dimensional consolidation. J. Appl. Phys. 12:155-164.

Biot, M.A. 1954. Theory of stress-strain relations in anisotropic viscoelasticity and relaxation phenomena. J. Appl. Phys. 25:1385-1391.

Biot, M.A. 1955. Variational principles in irreversible thermodynamics with application to viscoelasticity. Phys. Rev. 97:1463-1469.

Bonfield, W. and Datta, P.K. 1976. Fracture toughness of compact bone. J. Biomech. 9:131-134.

Bonfield, W. and Li, C.H. 1966. Deformation and fracture of bone. J. Appl. Phys. 37:869875.

Bonfield, W. and Li, C.H. 1967. Anisotropy of nonelastic Flow in Bone. J. Appl. Phys. 38:2450.

Bonfield, W.; Grynpas, M.D. and Young, R.J. 1978. Crack velocity and the fracture of bone. J. Biomech. 11:473-479. 
Bowman, S.M; Gibson, L.J.; Hayes,W.C. and McMahon, T.A.1999. Results from demineralized bone creep tests suggest that collagen is responsible for the creep behavior of bone. J. Biomech. Eng. 121:253-258.

Bowman, S.M.; Guo, X.E.; Cheng, D.W.; Keaveny, T.M.; Gibson, L.J.; Hayes,W.C. and McMahon, T.A.1998. Creep contribute to the fatigue behavior of bovine trabecular bone. J. Biomech. Eng. 120:647-654.

Boyde, A. 1980. Electron microscopy of the mineralizing front. Metabolic bone disease and related reseaech. 2(Suppl):69-78.

Budiansky, B and O'Connel, R.J. 1976. Elastic moduli of a cracked solid. Int. J. Solids Struct.12:81-97.

Budiansky, B., Hutchinson, J.W., Lambropoulus, J.C. 1983. Continuum theory of dilatant transformation toughening in ceramics. Int. J. Solids Struct. 19:337

Burr, BD; Milgrom, C; Boyd, RD; Higgins, WL; Robin, G; Radin, EL.1990. Experimental stress fractures of the tibia. J. Bone Joint Surg. 72B:370-375.

Burr, D.B. 1992. Remodeling and the repair of fatigue damage. Calc. Tiss. Int. 53:S75S81.

Burr, D.B. and Hooser, M. 1995. Alterations to the en bloc basic fuchsin staining protocol for the demonstration of microdamage produced in vivo. Bone. 17:431-433.

Burr, D.B. and Stafford, T. 1970. Validity of the bulk staining technique to separate artifactual from in-vivo bone microdamage. Clin. Orth. Rel. Res. 26:304-308.

Burr, D.B., Forwood, M.B., Schaffler, M.B. and Boyd, R.D. 1995. Trans. Orth. Res. Soc. 526. 
Burr, DB; Forwood, MR; Fyhrie, DP; Martin, RB; Schaffler, MB; Turner, CH. 1997. Bone microdamage and skeletal fragility in osteoporotic and stress fractures. J. Bone Mineral Res. 12:6-15.

Burr, DB; Martin, RB; Schaffler, MB; Radin, EL. 1985. Bone remodeling in response to in-vivo fatigue microdamage. J. Biomech. 18:189-200.

Burstein, A.H., Reilly, D.T. and Frankel, V.H. 1973. Failure characteristics of bone and bone tissue. In: "Perspectives in biomedical engineering. Ed. Kenedi, Macmillan, London.

Caler, W.E. and Carter, D.R. 1989. Bone creep-fatigue damage accumulation. J. Biomech. 22:625-635.

Callen, H.B. 1960. Thermodynamics, John Wiley New, York, NY.

Carter, D.R. and Caler, W.E. 1985. A cumulative damage model for bone fracture. J. Orth. Res. 3:84-90.

Carter, D.R. and Hayes, W.C. 1976. Bone Compressive Strength: The influence of Density and Strain Rate. Science 194:1174.

Carter, D.R. and Hayes, W.C. 1976a. Fatigue life of compact bone. I. Effects of stress amplitude, temperature and density. J. Biomech. 9:27-34.

Carter, D.R. and Hayes, W.C. 1977a. Compact bone fatigue damage. A microscopic examination. Clin. Orthop. 127:265-274.

Carter, D.R. and Hayes, W.C. 1977b. Compact bone fatigue damage.I. Residual strebgth and stiffness. J. Biomech. 10:323-337. 
Carter, D.R. and Caler, W.E. 1983. Cycle-Dependent and time-dependent bone fracture with repeated loading. J. Biomech. Eng.105:166-170.

Carter, D.R. and Caler, W.E. 1985. A cumulative Damagfe Model forBone Fracture. J. Orth. Res.3:84-90.

Carter, D.R. and Caler, W.E. 1989.Bone sreep-fatigue damage accumulation. J. Biomech. 22:625-635.

Carter, D.R., Caler, W.E., Spengler, D.M. and Frankel, V.H. 1981a. Fatigue behavior of adult cortical bone. The influence of mean strain range. Acta Orthop. Scandinav. 52. 481490.

Carter, D.R., Caler, W.E., Spengler, D.M. and Frankel, V.H. 1981b. Uniaxial fatigue of human cortical bone. The influence of tissue physical characteristics. J. Biomech. 14:461470 .

Carter, D.R., Hayes, W.C. and Schurman, D.J. 1976b. Fatigue life of compact bone.II. Effects of microstructure and density. J. Biomech. 9: 211-218.

Chamay, A. 1970. Mechanical and morphological aspect of experimental overload and fatigue in bone. J. Biomech. 3:263-270.

Chow, C.L and Wang, J. 1987. An anisotropic theory of continuum damage mechanics for ductile fracture. Eng. Fract. Mech. 27:547-558.

Coleman, B.D. 1964. Thermodynamics of materials with memory. Arch. Rational Mech. Anal. 17:1-46.

Coleman, B.D. and Gurtin, M.E. 1967. Thermodynamics with internal state variables. $J$. Chem Phys. 47:597-613. 
Coleman, B.D. and Mizel, V.J. 1964. Existence of coloric equations of state in thermodyanamics. J. Chem. Phys. 40:1116-1125.

Coleman, B.D. and Noll, W. 1963. The thermodynamics of elastic materials with heat conduction and viscosity. Arch. Rational Mech. Anal. 13:167-178.

Cowin, S.C. 1999. Bone Poroelasticity. J. Biomech. 32:217-238.

Cowin, S.C. and Hegedus, D.M. 1976. Bone Remodeling I: A Theory of Adaptive Elasticity. J. Elasticity, 6:313-325.

Cowin, S.C.; Weinbaum, S. and Zeng, Y. 1995. A case for bone canaliculi as the anatomical site of strain-generated potentials. J. Biomech. 28:1281-1296.

Currey, J.D. 1964. Three analogies to Explain the Mechanical Properties of Bone. Biorheology. 2:1

Currey, J.D. 1969. The mechanical consequences of variation in the mineral content of bone. $2: 1$.

Currey, J.D. 1969. The relationship between the stiffness and the mineral content of bone. J. Biomech. 2:477.

Currey, J.D. 1984. The Mechanical Adaptations of Bones. Princeton University Press, Princton, NJ.

Davison, L and Stevens, A.L. 1973. Thermodynamic constitution of spalling elastic bodies. J. Appl. Phys. 44:668-674.

Devas, M.B. 1975. Stress fractures. Churchill-Livingston. London. 
Doty, S.D. and Schofield, B.H. 1971. Metabolic and structural changes within osteocytes of rat bone. In: Calcium, parathyroid hormone and the calcitonins. Ed. Talmage and Menson. Experta Medica, Amsterdam.

Dougdill, J.W. 1929. Beruchnung der Fliessgrenze von Mischkristallen. Z. Angew. Math. Mech. 59:427.

Enlow, D.H. 1969. The bone of reptiles. In: Biology of the reptilla, ed. C Gans. Voll:4580, Academic Press, NY.

Evans, F.G. 1957. Stess and strains in bone. Thomas, Springfield, IL.

Evans, FG and Lebow, M. 1957. Strength of human compact bone under repetitive loading. J. Appl. Physiol. 10:127-130.

Faber, K.T. and Evans, A.G. 1983a. Crack deflection processes-I. Theory. Acta Metall. 31:565.

Faber, K.T. and Evans, A.G. 1983a. Crack deflection processes-I. Experiment. Acta Metall. 31:577.

Ferry, J.D. 1980. Viscoelastic Properties of polymers.( $3^{\text {rd }}$ ED)John Wiley \& Sons, New York, NY.

Fondrk, M.T., Bahniuk, E.H. and Davy, D.T. 1999. A Damage Model for nonlinear tensile behavior of cortical bone. J. Biomech. Eng. 121:533-541.

Fondrk, M.T., Bahniuk, E.H. and Davy, D.T. 1999.Inelastic Strain Accumulation in Cortical Bone During Rapid Transient Tensile Loading. J. Biomech. Eng. 121:616-621. 
Fondrk, M., Bahniuk, E., Davy, D.T. and Michaels, C. 1988. Some viscoplastic characteristics of bovine and human cortical bone. J. Biomech. 21:623-630.

Forwood, M.R. and Parker, A.W. 1989. Microdamage in response to repetitive torsional loading in the rat tibia. Calc. Tiss. Int. 45:47-53.

Frost, H.M. 1960. Presence of microscopic cracks in vivo in bone. Bulletin Henry Ford Hosp. 8:25.

Frost, H.M. 1963a. Bone remodeling dynamics. Charles C. Thomas. Springfield, IL.

Frost, H.M. 1963b. Dynamics of bone remodeling. In Bone Biodynamics (Ed. Frost, HM). Charles C. Thomas. Sprinfield, IL.

Frost, H.M. 1964. The Laws of Bone Structure., Charles C. Thomas, Springfield, IL.

Frost, H.M. 1966. Bone dynamics in osteoporosis and osteomalcia. Charles C. Thomas. Sprinfield, IL.

Frost, H.M. 1969. Tetracycline-based histological analysis of bone remodeling. Calc. Tiss. Res. 3:211-237.

Fung, Y.C. 1965. Fundamentals of Solid Mechanics. Englewood Cliffs, Prentice Hall, NJ.

Fyhrie, D.P. and Carter, D.R. 1986. A Unifying Principle Relating Stress to Trabecular Bone Morphology. J. Orth. Res. 4:304-317.

Geyer, M., Sander-Beuermann, A., Wegner, U. and Wirth, C.J. 1993. Stress reactions and stress fractures in the high performance athlete. Causes, diagnosis and therapy. Unfallchirurg. 96:66-74. 
Hart, R.T. and Davy, D.T. 1989. Theory of Bone Modeling and Remodeling. In :Bone Mechanics, edited by S.C. Cowin, CRC Press,. Boca Raton, FL.

Hashin, Z. 1988. The differential scheme and its application to cracked materials. $J$. Mech. Phys. Solids. 36:719-734.

Hashin, Z. and Rosen, B.W. 1964. The elastic moduli of fiber reinforced materials. $J$. Appl. Mech. 31:223-232.

Hashin, Z. and Shtrikman, S. 1963. A variational approach to the theory of elastic behavior of multiphase materials. J. Mech. Phys. Solids. 11:127.

Hillsley, M.V. and Frangos, J.A. 1994. Review: Bone Tissue Engineering: The Role of Interstitial Fluid Flow. Biotechnol. Bioengineer. 43:573-581.

Hillsley, M.V. and Frangos, J.A. 1996. Osteoblast hydraulic conductivity is regulated by calcitonin and parathyroid hormone. J. Bone Min. Res. 11:114-124.

Hoenig, A. 1979. Elastic moduli of a non-randomly cracked body. Int. J. Solids Struct. 15:137-154.

Jepsen, K.J. and Davy, D.T. 1997. Comparison of damage accumulation measures in human cortical bone. J. Biomech. 30:891-894.

Jepsen, K.J., Davy, D.T. and Krzypow, D.J. 1999. The role of the interlamellar interface during torsional yielding of human compact bone. J. Biomech. 32:303-310

John, H., Burckhard, A. and Gachter, A. 1994. Fatigue fracture: a frequently overloaded injury? Helv. Chir. Acta. 60:551-555. 
Johnson, L. C. 1964. Morphologic analysis in pathology: The kinetics of disease and general biology in bone. In Bone Biodynamics (Ed. Frost, HM). Charles C. Thomas. Sprinfield, IL.

Jones, BH; Harris, JM; Vinh, TN; Rubin, C. 1989. Exercise-induced stress fractures and stress reactions of bone: Epidemiology, etiology and classification. Exers. Sport. Sci. Rev. 17:379-422.

Ju, J.W. 1989. On Energy based coupled elastoplastic damage theories: Constitiutive modeling and computational aspects. Int. J. Solids Struct. 25(7):803-833.

Kachanov, L.M. 1958. Time of the rupture process under creep conditions. Izv. AN.SSR, Otd. Tekh. Nauk. 8:26-31.

Kachanov, M. 1992. Effective elastic properties of cracked solids: Critical review of some basic concepts. Appl. Mech. Rev. 45:304-335.

Katz, J.L. 1971. Hard tissue as a composite material. 1. Bounds on the elastic behavior. In: Mechanical Properties of Bone. Ed. S.C. Cowin, AMD Vol 45.

Katz, J.L. 1971. Hard Tissue as a Composite Material.I. Bounds on the Elastic Behavior. J. Biomech. 4:455.

Kestin, J and Rice, J.R. 1970. Paradoxes in application of thermodynamics to strained solids. In "A critical review of thermodynamics." Ed: Stuart, Brainard and Gal-Or. Baltimore, Mono Book, MD.

Knets, I.V. and Vilks, Y.K 1975. Creep of compact human bony tissue under tension. Polymer Mech. 11:543-547. 
Knets, I.V., Krauya, U.E., Layzan, Y.B. 1976. Some features of bone tissue deformation under unloading and repeated loading. Polymer Mech. 12:779-786.

Knothe Tate, M.L.; Niederer, P. and Knothe, U. 1998. In vivo tracer transport through the lacunae-canalicular system of rat bone in an environment devoid of mechanical loading. Bone 22:107-117.

Korostoff, E and Salzstein, R. 1981. Evidence for the piezoelectric domain model for stress-generated potentials in bone. Trans. Bioelectrical Repair and Groath Soc. Piladelphia. 1:6.

Korostoff, E. 1977. Stress-generated potentials in bone:Relationship to piezoelectricity in collagen. J. Biomech. 10:41

Korostoff, E. 1979. A linear piezoelectric model for characterizing stress-generated potentials in bone. J. Biomech. 12:335-347.

Krajcinovic, D and Fonseka, G.U. 1981. The continuous damage theory of brittle materials. J Appl. Mech. 48:809-824.

Krajcinovic, D. 1985. Continuum damage mechanics revisited: basic concepts and definitions. J. Appl. Mech. 52:829-834.

Krenchel, H. 1964. Fiber reinforcements. Academic Forlag, Copenhagen.

Lacroix, P. 1974. The internal remodeling of bones. In The biochemistry and physiology of bone. Ed. Bourne, G.H. Academic Press, NY.

Lakes, R 1993. Materials with Structural Hierarchy. Nature. 361(11):511-515. 
Lakes, R.S. and Katz, J.L. 1979. Viscoelastic properties of wet cortical bone-II. Relaxation mechanisms. J. Biomech. 12:679-687.

Lakes, R.S.; Katz, J.L. and Sternstein, S.S. 1979. Viscoelastic properties of wet cortical bone-I. Torsional and Biaxial studies. J. Biomech. 12:657-678.

Lakes, R.S.and Katz, J.L. 1979. Viscoelastic properties of wet cortical bone-III. A nonlinear constitutive equation. J. Biomech. 12:689-698.

Lanyon, L.E. 1984. Functional Strain as a Determinant for Bone Remodeling. Calcif. Tiss. Int. 36:S56-S61.

Lathi, B.P. 1992. Linear systems and signals. Berkley-Cambridge Press, Carmichael, CA.

Lemaitre, J and Chaboche, J.L. 1975. A non-linear model of creep-fatigue damage accumulation and interaction. In "Mechanics of viscoelastic media and bodies." Ed. Hult. Springer-Verlag, NY.

Lemaitre, J. 1985. A continuum damage mechanics model for ductile fracture. J Eng. Mat. Technol. 107:83-89.

Lemaitre, J. and Chaboche, J.L. 1990. Mechanics of solid materials. Cambridge University Press, UK.

Lewis, J.L. and Goldsmith, W. 1975. The dynamic fracture and prefracture response of compact bone by split Hopkinson bar methods. J. Biomech. 8:27-40.

Li, G; Bronk, J.T.; An, K.N. and Kelly, P.J. 1987. Permeability of cortical bone of canine tibiae. Microcirculation Res. 34: 302-310.

Lubliner, J. 1972. Int. J. Nonlinear Solid Mech. 7:237-254. 
Lin, G.H. and Lai, K.K.L. 1993 J. Mater. Sci. 28:5340-5246.

Mai, YW. 1988. Fracture resistance and fracture mechanics of engineering materials. Mater. Forum. 11:289.

Maitra, RS; Johnson, DL. 1997. Stress fractures: Clinical history and physical examination. Clin. Sports. Med. 16:259-274.

Martin, B. 1994. Mathematical model for the mineralization of bone. J. Orth. Res.12:375383.

Martin, R.B. and Burr, D.B. 1982. A Hypothetical Mechanism for the Stimulation of Osteonal Remodeling by Fatigue Damage. J. Biomech. 15:137-139.

Martin, R.B. and Burr, D.B. 1982. A hypothetical mechanism for the stimulation of osteonal remodeling by fatigue damage. J. Biomech. 15:137-139.

Mashiba, T., Hirano, T., Turner, C.H., Forwood, M.R., Johnston, C.C. and Burr, D.B. 2000. Suppressed bone turnover by bisphosphonates increases microdamage accumulation and reduces some biomechanical properties. J. Bone Miner. Res. 15:613620.

McCarthy, I.D. and Yang, L. 1992. A distributed model of exchange processes within the osteon. Physics in Med. And Biol. 36:1331-1340.

Melnis, A.E. and Knets, I.V. 1981. Effect of moisture on the mechanical behavior of compact bone. Mech. Compos. Mater. 17:219-226.

Melnis, A.E. and Knets, I.V. 1981a. Age related changes in tensile creep properties of human compact bone tissue. Mech. Of Comp. Mat. 17:495-501. 
Melnis, A.E. and Knets, I.V. 1981b. Effect of moisture on the mechanical behavior of compact bone. Mech. Of Comp. Mat. 17:219-226.

Melvin, J.W. and Evans. F.G. 1973. Crack propagation in bone. Biomat. Sympos. AMD 2. Ed. Fung, Y.C., ASME, N.Y.

Meyer, S. 1993. Stress fractures of the foot and leg. Clin. Sports. Med. 12:395.

Miller, S.C. and Jee, W.S.S. 1992. Bone lining cells. In. Bone. Vol. 4 Bone metabolism and mineralization. Ed. Hall, B.K., CRC Press, Boca Raton.

Monteleone, G. 1995. Stress fractures in the athlete. Orthop. Clin. North. Am 26:423.

Mori, S. and Burr, D.B. 1993. Increased intracortical remodeling following fatigue damage. Bone. 14:103-109.

Mori, T and Tanaka, K. 1973. Average stress in matrix and average energy of materials with misfitting inclusions. Acta Metall. 31.571-574.

Morris, J.M. and Blickenstaff, L.D. 1967. Fatigue fractures: A clinical study. Charles C. Thomas. Sprinfield, IL.

Morris, M.A.; Lopez-Curato, T.A.; Hughes, S.P.F.; An, K.N.; Bassingthwaighte, J.B. and Kelly, P.J. 1982. Fluid spaces in canine bone and marrow. Microvasc. Res.23:188-200.

Murakami, S and Ohno, N. 1981. A continuum theory of creep and creep damage. In "Creep in structures." Ed. Pointer and Hayrhaust. Springer-Verlag, NY.

Neuman, M.W 1982. Bone fluid equilibrium. Calc. Tiss. Int. 34:117-120. 
Neuman, W.F. and Neuman, M.W. 1958. The chemical dynamics of bone. University of Chicago Press, Chicago, IL.

Norman, T.L. and Wang, Z. 1997. Microdamage of human cortical bone: incidence and morphology in long bones. Bone. 20:375-379.

Norman, T.L.; Navargikar, S.V. and Burr, D.B. 1996. Resistance to crack growth in human cortical bone is greater in shear than in tension. J. Biomech. 29:1023-1031.

Norman, T.L.; Vashishth, D. and Burr, D.B. 1991. Mode I fracture toughness of human bone. Adv. in Bioeng., ASME.

Norman, T.L.; Vashishth, D. and Burr, D.B. 1995. Fracture toughness of human bone under tension. J. Biomech. 28:309-320.

Nowinski, J.L. and Davis, C.F. 1970. A model of the human skull as a poroelastic spherical shell subjected to a quasistatic load. Math. Biosc. 8:397-416.

O’Brien, T.K. and Reifsnider, K.L. 1977. Fatigue damage: Stiffness/strength comparisons for composite materials. J. Testing and Evaluation. 5:384-393.

O'Connel, R.J. and Budiansky, B. 1977. Viscoelastic properties of fluid-saturated scracked solids. J. Geophys. Res. 82:5719-5735.

Parsamian, G.P. and Norman, T.L. 2001. Diffuse damage accumulation in the fracture process zone of human cortical bone specimens and its influence on fracture toughness. $J$. Mat. Sci.: Mat. Med. (in press).

Parsamian, G.P., Norman, T.L. and Kish, V.L. 2001. Damage Evolution and Fracture Behavior of Human Compact Bone Specimens in 3-Point Bending. $47^{\text {th }}$ Annual Meeting of Orthopedic Research Society, San Francisco, CA, February 25-28. 
Parsamian, G.P., Norman, T.L. and Kish, V.L. 2000. The Mechanics of Ultrastructural Damage occurrence and Its Influence on The Fracture Toughness of Human Cortical Bone. $46^{\text {th }}$ Annual Meeting of Orthopedic Research Society, Orlando, FL, March 12-15.

Piekarski, K. 1970. Fracture of bone. J. Appl. Phys. 41:215-233.

Piekarski, K. 1973. Analysis of Bone as a Composite Material. Int. J. Eng. Sci. 11(6A):557.

Pope, M.H. and Outwater, J.O. 1972. The fracture characteristics of bone substance. J. Biomech. 5:457-465.

Rabotnov, Y.N. 1963. Damage from creep (in Russian). Zhurn. Prikl. Mekh. Tekhn. Phys. 2:113-123.

Rabotnov, Y.N. 1980. Elemnts of hereditary solid mechanics. Mir. Moscow.

Rauber, A.A. 1876. Elasticitat und Festigkeit der Knochen. Anatomisch-Physiologische Studie. Engelmann, Leipzig.

Reuss, A. 1929. Beruchnung der Fliessgrenze von Mischkristallen. Z. Angew. Math. Mech. 9:48.

Rho, J.Y., Kuhn-Spearing, L. and Zioupos, P. 1998. Mechanical Properties and the Hierarchical Structure of Bone. Med. Eng. Phys. 20:91-102.

Rice J.R. 1971. Inelastic constitutive relations for solids. An internal variable theory and its application to metal plasticity. J. Mech. Phys. Solids. 19:433-455.

Rodan, G.A., Bourret, L.A., Havey, A. and Mensi, T. 1975. Cyclic AMP and cyclic GMP: Mediators of the mechanical effects on bone remodeling. Science. 189. 467-469. 
Salzstein, R.A. and Pollak, S.R. 1987. Electromechanical potentials in Cortical Bone-II. Experimental Analysis. J. Biomech. 20:271-280.

Salzstein, R.A.; Pollak, S.R.; Mak, A.F.T. and Petrov, N. 1987. Electromechanical Potentials in Cortical Bone-I. A Continuum Approach. J. Biomech. 20:261-270.

Sasaki, N; Nakayama, Y; Yoshikawa, M and Enyo, A. 1993. Stress relaxation function of bone and bone collagen. J. Biomech. 26:1369-1376.

Schaffler, M.B., Boyce, T.M. and Fyhrie, D.P. 1996. Tissue and matrix failure modes in human compact bone during tensile fatigue. Trans. Orth. Res. Soc. 21:57.

Schaffler, M.B., Choi, K. and Milgrom, C. 1995. Aging and matrix microdamage accumulation in human compact bone. Bone. 17:521-525.

Schaffler, M.B., Radin, E.L. and Burr, D.B. 1990. Long term fatigue behavior of compact bone at low strain magnitude and rate. Bone. 11:321-326.

Schaffler, MB; Pitchford, WC; Choi, K; Riddle. JM. 1994. Examination of compact bone microdamage using back-scattered electron microscopy. Bone 15:483-488.

Schapery, R.A. 1964. J. Appl. Phys. 35:1451-1465.

Schapery, R.A. 1969. Purdue University ReportNo. AA\&ES 69-2

Schapery, R.A. 1981. On viscoelastic deformation and failure behavior of composite materials with distributed flaws. Adv. Aerospace Str. Mats. Ed: Wang and Renton, ASME, NY. 
Schapery, R.A. 1984. Correspondence principles and a generalized J-Integral for large deformation and fracture analysis of viscoelastic media. Int. J. Fracture 25:195-223.

Scott, G.C. and Korostoff, E. 1990. Oscillatory and Step Response to Electromechanical Phenomena in Human and Bovine Bone. J. Biomech. 23:127-143.

Sedlin, E.D. 1965. A rheologic model for cortical bone. Acta Orthop. Scandinav. Supplementum 83, Copenhagen.

Simo, J.C. and Ju, J.W. 1987. Strain- and stress-based continuum damage models. Int. J. Solids Struct. 23:821-869.

Smith, R and Keiper, D. 1965. Dynamic measurement of viscoelastic properties of bone. Am J. Med. Elec. 4:156-160.

Smrcine, C. 1991. Stress fractures in athletes. Nurs. Clin. North. Am. 26:159.

Soares, A.M.V.; Arana-Chavez, V.E.; Reid, A.R. and Katchburian, B. 1992. Lanthanum tracer and freeze-fracture studies suggest that compartmentization of early bone matrix may be related to initial mineralization. J. Anatomy. 181. 343-356.

Starkebaum, W; Pollak, S.R. and Korostoff, E. 1979. Microelectrode Studies of StressGenerated Potentials in Four-Point Bending of Bone. J. Biomed. Mat. Res. 13:729-751.

Sterling, J. 1992. Stress fractures in the athlete: Diagnosis and management. Sports Med. $14: 336$.

Swanson, S.A.V., Freeman, M.A.R. and Day, W.H. 1971. The fatigue properties of human cortical bone. Medical and Biological Engineering. 9:23-32. 
Tanaka, T and Sakano, A. 1985. Differences in permeability of microperoxidase and horseradish peroxidase into the alveolar bone of developing rats. J. Dent. Res. 64:870876.

Tschantz, P and Rutishauser, F. 1967. La surcharge, mecanique de l'os vivant. Annales d'Anatomie Pathologique. 12:223-248.

Vashishth, D., Behiri, J.C., and Bonfield, W. 1997. Crack growth resistance in cortical bone: Concept of microcrack toughening. J. Biomech. 30:763-769.

Verborgt, O, Gibson, G.J. and Schaffler, M.B. 2000. Loss of osteocyte integrity in association with microdamage and bone remodeling after fatigue in vivo. J. Bone Miner. Res. 15:60-67.

Voigt, W. 1928. Lehrbuch der Kristallphysik. Tenbner, Leipzig.

Weinbaum, S.; Cowin, S.C. and Zeng, Y. 1994. A model for the excitation of osteocytes by mechanical loading-induced bone fluid shear stresses. J. Biomech. 27:339-360.

Weinbaum, S.; Cowin, S.C. and Zeng, Y. 1994. Excitation of Osteocytes by Mechanical Loading-Induced Bone Fluid Shear Stress. J. Biomech. 27:339-360.

Weitsmann, Y. 1988. A continuum damage model for viscoelastic materials. J. Appl. Mech. 55:773-780.

Williams, G and Watts, D.C. 1970. Non-symmetrical dielectric relaxation behavior arising from a simple empirical decay function. Trans. Faraday Soc. 66:80-85.

Wolff, J. 1892. Das Gesetz der Transformation der Knochen, Hirschwald, Berlin.

Wolff, J. 1986. The Law of Bone Remodeling, Springer, Berlin. 
Wright, T.M. and Hayes, W.C. 1977. Fracture mechanics parameters for compact bone,effects of density and specimen thickness. J. Biomech. 10:419-430.

Yasuda, I. 1953. Fundamental aspects of fracture treatment. J. Kyoto Med. Soc. 4:395406.

Yazdani, S. 1993. On a class of continuum damage mechanics. Int. J. Damage. Mech. 2:162-176.

Yeni, Y.N.; Brown, C.U. and Norman, T.L. 1999. Influence of bone composition and apparent density on fracture toughness of human femur and tibia. Bone 22:

Yeni, Y.N.; Brown, C.U.; Wang, Z. and Norman, T.L. 1997. The influence of bone morphology on fracture toughness of the human femur and tibia. Bone. 21:453-459.

Zhang, D.; Weinbaum, S. and Cowin, S.C. 1998. Estimates of the peak pressures in the bone pore water. J. Biomech. Eng.

Zioupos, P, Wang, X.T. and Currey, J.D. 1996. The accumulation of fatigue micridamage in human cortical bone of two different ages in vitro. Clin. Biomech. 11:365-375.

Zioupos, P. and Casinos, A. 1998. Cumulative damage and the response of human bone in two-step loading fatigue.31:825-833.

Zioupos, P., Wang, X.T. and Currey, J.D. 1996. Experimental and theoretical quantification of the development of damage in fatigue tests of bone and antler. $J$. Biomech. 29:989-1002.

Zioupos, P; Currey, JD. 1994. The extent of microcracking and the morphology of microcracks in damaged bone. J. Mater. Sci. 29:978-986. 